\title{
Evaluating Psychosocial Variables and their Link to Hypertension Using Mindfulness-Based Stress Reduction
}

by

Kimberly Blom

A thesis submitted in conformity with the requirements

for the degree of Master of Science

Institute of Medical Science

University of Toronto

(C) Copyright by Kimberly Blom 2013 


\title{
Evaluating Psychosocial Variables and their Link to Hypertension Using Mindfulness-Based Stress Reduction
}

\author{
Kimberly Blom \\ Masters of Science \\ Institute of Medical Science \\ University of Toronto
}

2013

\section{Abstract}

Previous research has reported positive associations between anger, perceived stress and blood pressure. These associations have largely been based on cross-sectional data and a small number of longitudinal works. Using a prospective longitudinal cohort design, this study more directly tested the relationships between anger, perceived stress and blood pressure by using a psychological therapeutic intervention (mindfulness-based stress reduction) to manipulate anger and perceived stress. Anger and perceived stress were in turn evaluated for association with blood pressure. Despite improvements in psychosocial functioning and reductions in blood pressure, findings from this study failed to demonstrate an association between change in anger or perceived stress with change in daytime or 24-hour ambulatory blood pressure. A model where these variables interact indirectly via stress coping mechanisms or health behaviours may be the linking mechanism in this study. Results from this thesis have contributed evidence to a divided field dominated by cross-sectional research. 


\section{Acknowledgments}

Many wonderful people have helped with the completion of this thesis. I would first like to express tremendous gratitude and thanks to my supervisors, Dr. Sheldon Tobe and Dr. Robert Nolan. Their kindness, expertise and dedication helped make this master's journey an exceptional experience. I would also like to express my most sincere thanks and appreciation to my graduate advisors Dr. Jane Irvine and Dr. Gillian Einstein. Their support and guidance have contributed immensely to both my academic and professional development. I am forever grateful for their support.

I would also like to thank the following people who made my experience as a graduate student memorable: Nancy Perkins, Martha Agelopoulos and Tiffany Richards for their unwavering support and positivity, Roza Bulyaki and Stacy Knox for their helping hands, Alex Kiss and Marko Katic for their statistical expertise, Dr. Brian Baker for his excellent insights and Marika Galadza for her departmental support. I would also like to thank all of our study participants and collaborators on the HARMONY trial, for without their contributions this project would not have been possible. Thank you to the Queen Elizabeth II Graduate Scholarship in Science and Technology, the Ontario Graduate Scholarship and the Heart and Stroke Foundation for their funding contributions to this project and the HARMONY trial.

Finally, I would like to express my deepest thanks to my family and friends, especially my parents Bart and Katrina. They have witnessed the highs and lows of this journey, both inside and outside of graduate school. I would not be where I am today were it not for their unconditional love and support. 


\section{Table of Contents}

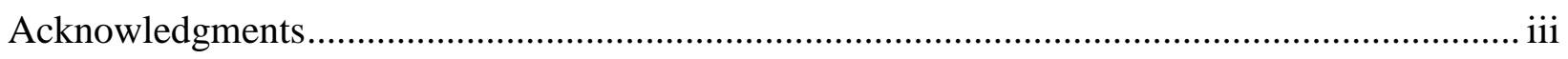

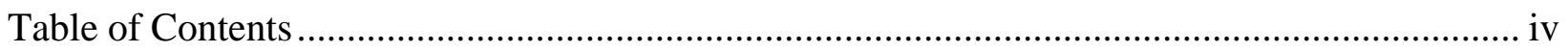

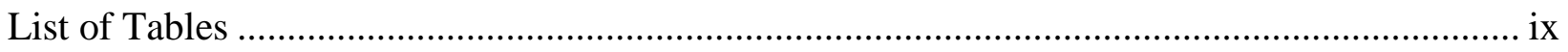

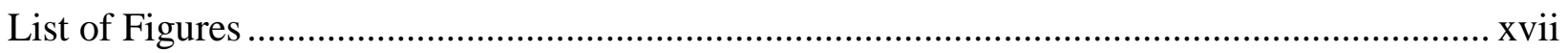

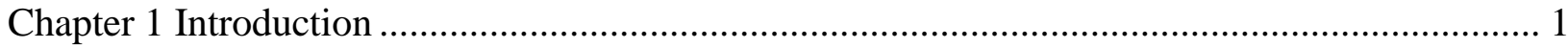

1 Hypertension: A Global Pandemic......................................................................................... 1

1.1 Risk Factors for Hypertension .............................................................................. 1

1.2 Pathophysiological Evidence \& Hypothesized Casual Pathways - Linking

Psychosocial Variables \& Blood Pressure .......................................................................... 2

1.3 Anger \& Perceived Stress as Psychosocial Risk Factors ........................................................ 4

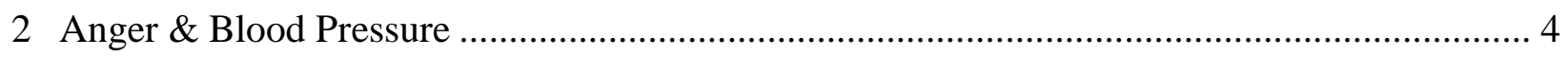

2.1 Historical Context \& Definitions ..................................................................................... 4

2.2 Evidence to Date on Anger \& Blood Pressure ……............................................................... 7

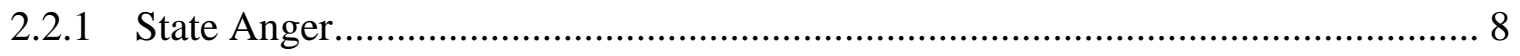

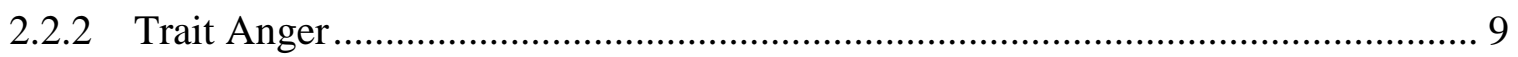

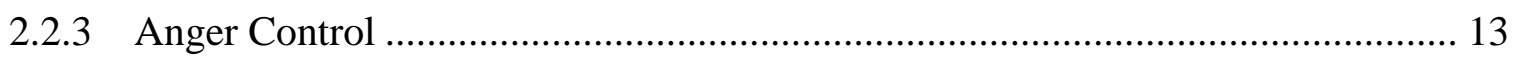

2.2.4 Anger Expression...................................................................................... 13

2.2.4.1 Anger Expressed In ........................................................................ 14

2.2.4.2 Anger Expressed Out....................................................................... 18

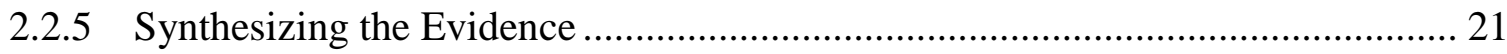

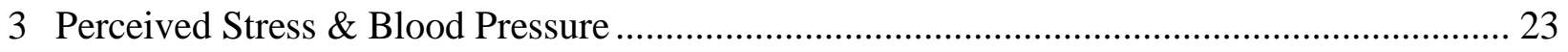

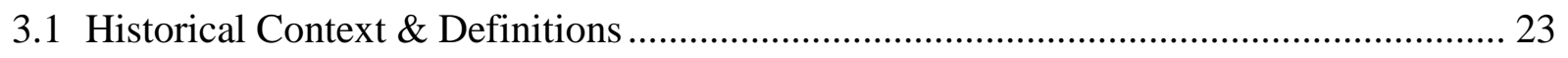

3.2 Evidence to Date on Perceived Stress \& Blood Pressure ................................................... 26

3.2.1 Blood Pressure \& Validated Measures of Perceived Stress.................................. 26

3.2.2 Blood Pressure \& Non-Validated Measures of Perceived Stress ......................... 28 


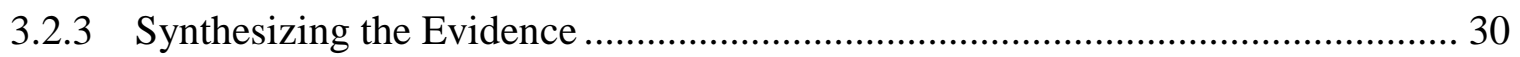

4 Moving Forward: Prospective Longitudinal Cohort Designs ……………………….............. 30

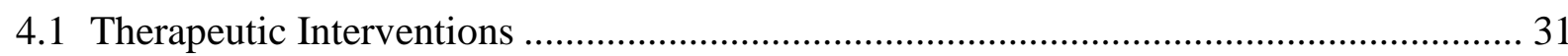

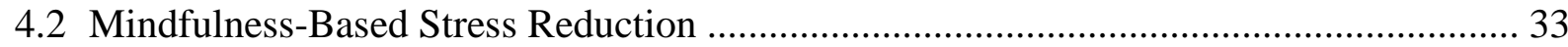

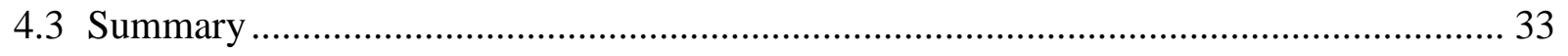

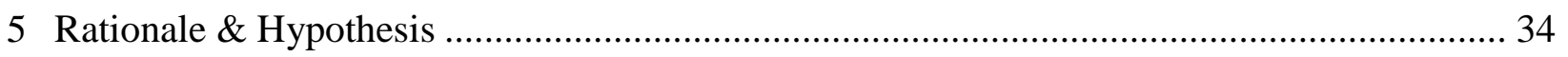

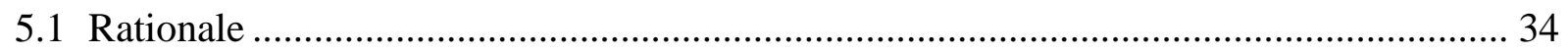

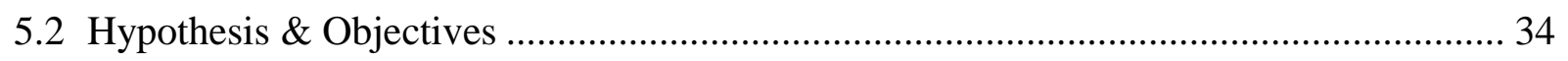

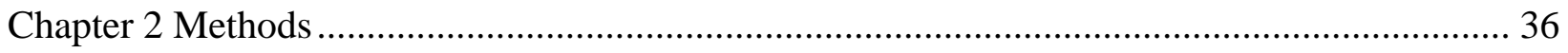

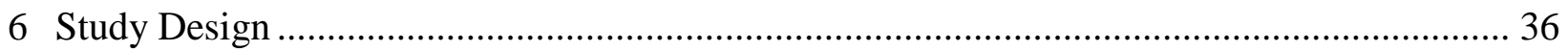

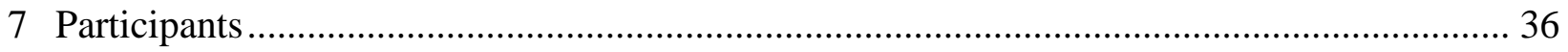

8 Screening \& Blood Pressure Measurement.................................................................................. 37

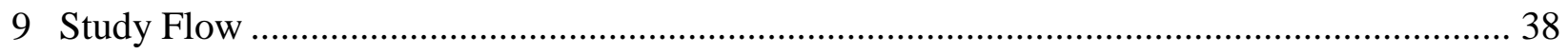

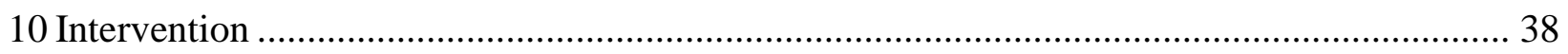

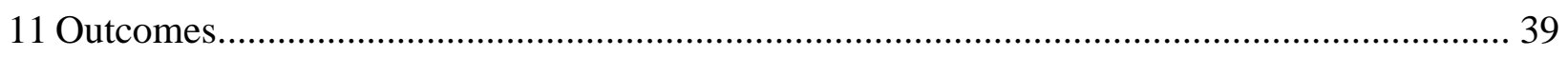

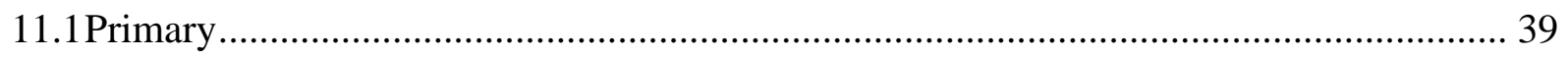

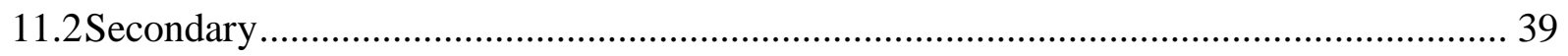

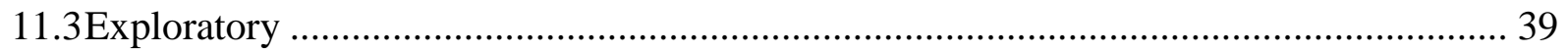

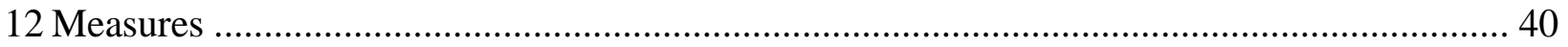

12.1 State Trait Anger Expression Inventory (STAXI) ……….............................................. 40

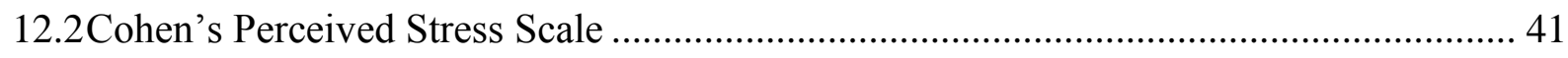

12.3 Ambulatory Blood Pressure Monitoring ......................................................................... 42

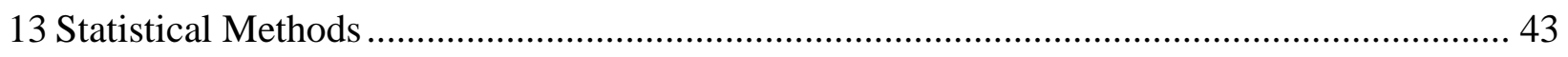

13.1 Baseline Correlations ................................................................................................. 43

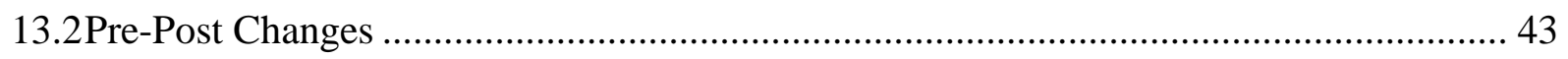

13.3Psychometric Properties............................................................................................ 44 


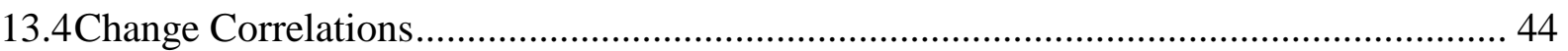

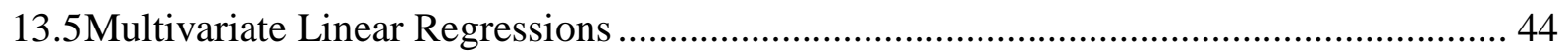

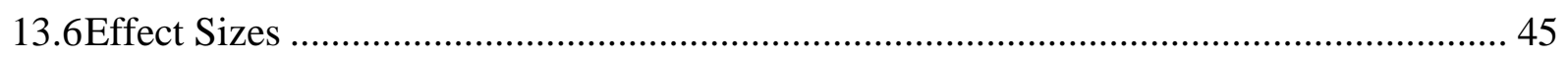

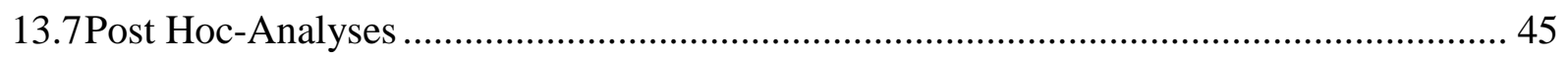

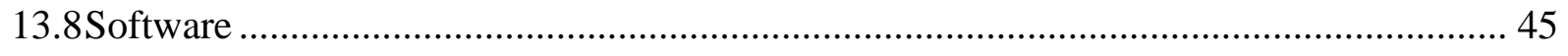

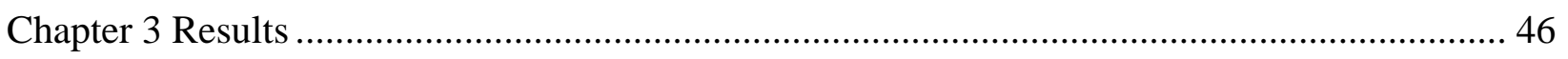

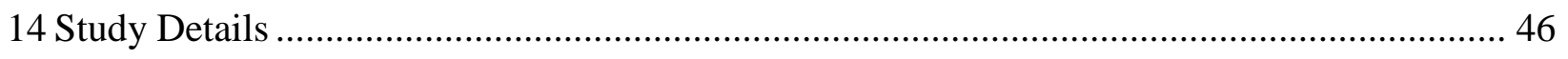

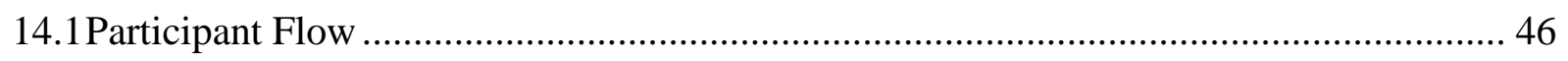

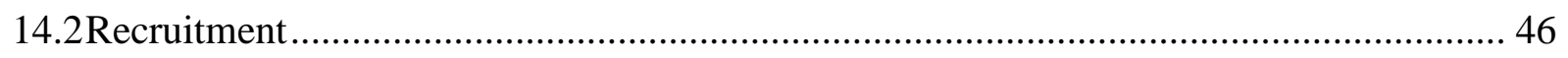

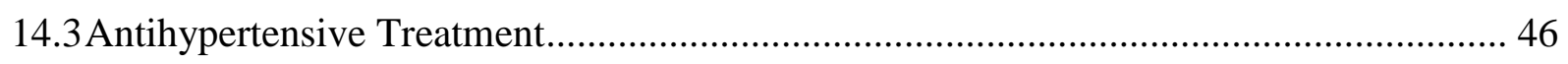

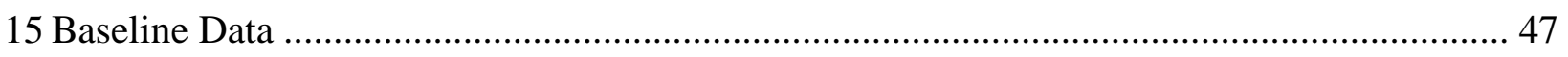

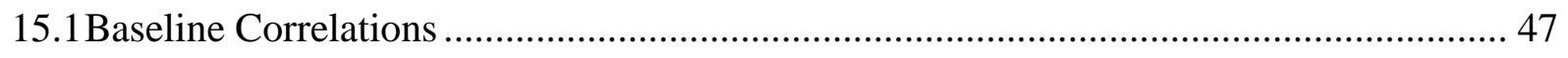

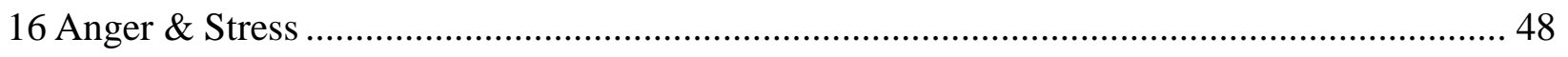

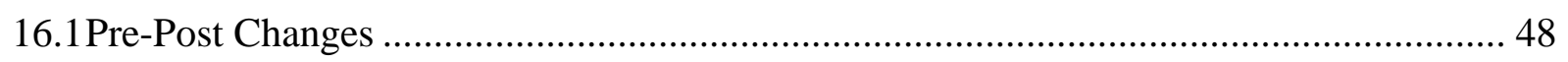

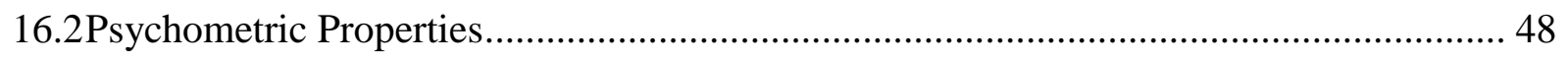

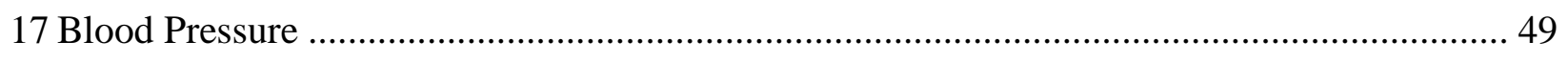

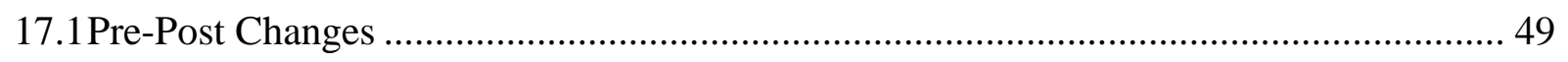

17.2Change Correlations with Psychosocial Measures ............................................................ 49

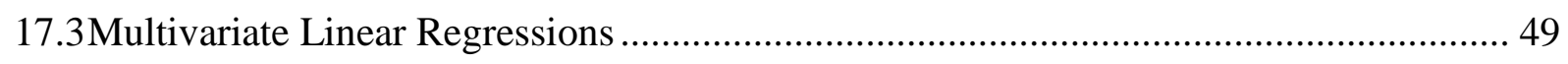

17.3.1 Primary Hypotheses: Primary Psychosocial Measures \& 24-hour and Daytime Blood Pressure ………………………………………................................. 50

17.3.2 Secondary Hypotheses: Primary Psychosocial Measures \& Nighttime Blood

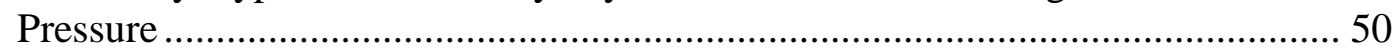

17.3.3 Exploratory Hypotheses: Exploratory Psychosocial Measures \& 24-hour, Daytime and Nighttime Blood Pressure ………………….................................. 51

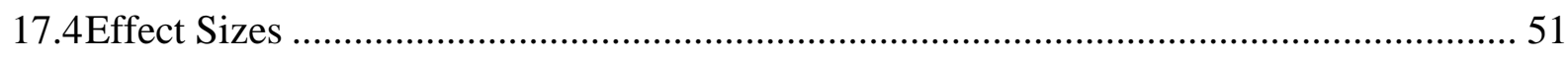

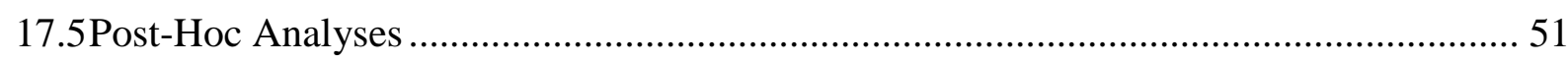




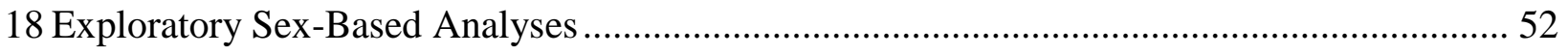

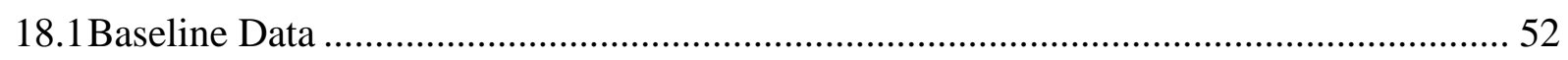

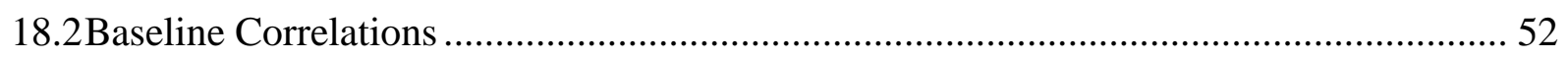

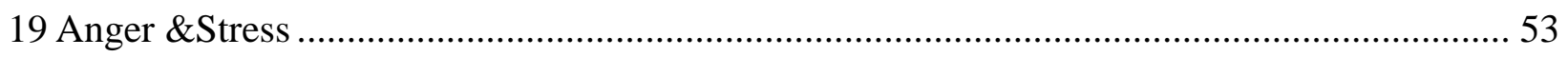

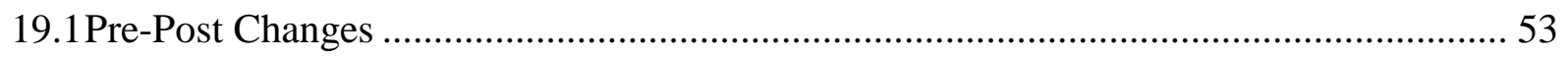

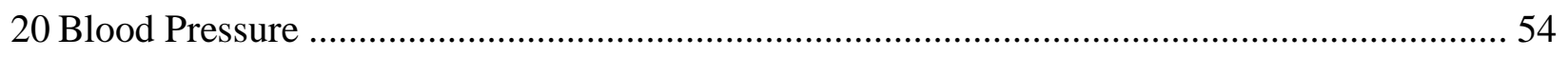

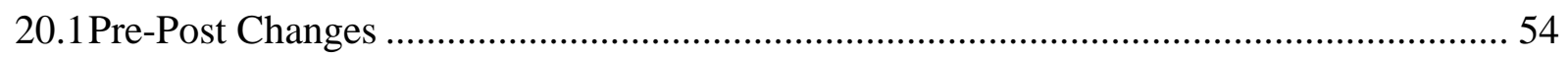

20.2Change Correlations with Psychosocial Measures ............................................................... 54

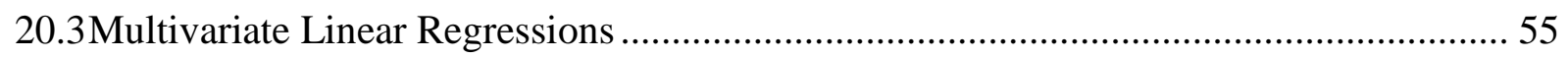

20.3.1 Primary Hypotheses: Primary Psychosocial Measures \& 24-hour and Daytime

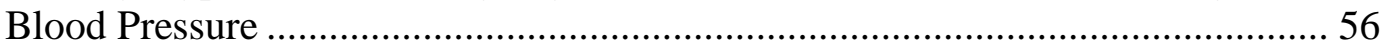

20.3.2 Secondary Hypotheses: Primary Psychosocial Measures \& Nighttime Blood

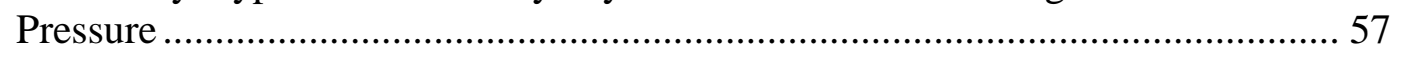

20.3.3 Exploratory Hypotheses: Exploratory Psychosocial Measures \& 24-hour, Daytime and Nighttime Blood Pressure …………………………….................. 58

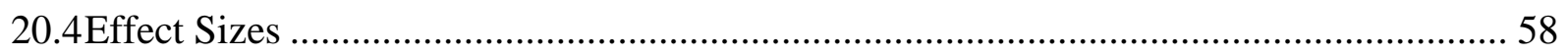

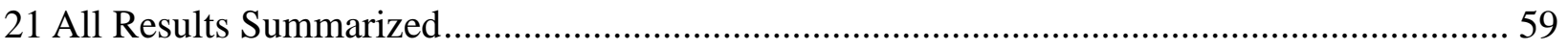

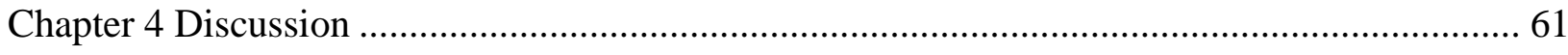

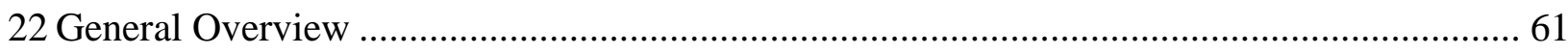

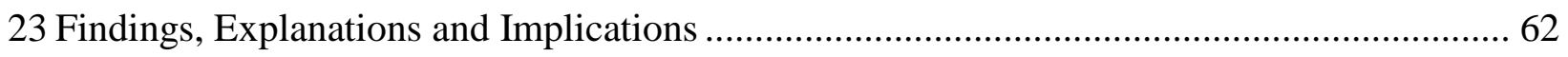

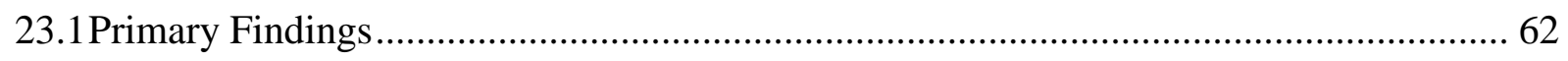

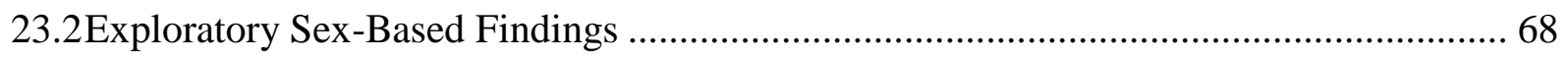

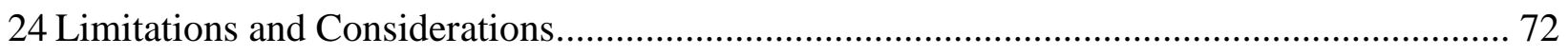

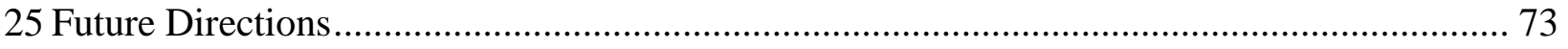

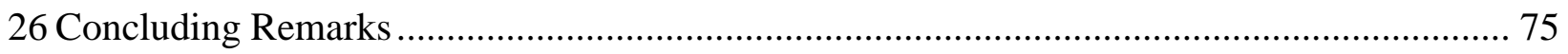

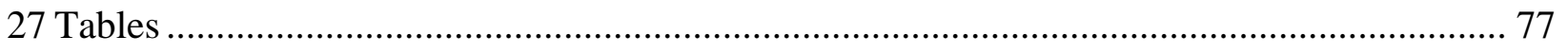

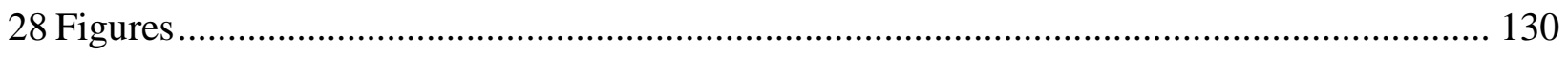




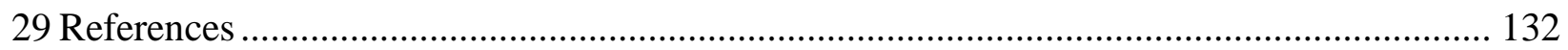




\section{List of Tables}

Table number. Description.

Page

Table 1. Studies examining state anger and blood pressure

Table 2. Studies examining trait anger and blood pressure

Table 3. Studies examining anger control and blood pressure

Table 4. Methodological inconsistencies in the field of anger expression and blood pressure research

Table 5. Studies examining anger expression and blood pressure

Table 6. Studies examining perceived stress and blood pressure

Table 7. Study inclusion and exclusion criteria(adapted from the HARMONY protocol manuscript $)^{1}$

Table 8. Brief overview of the STAXI-2 scales and subscales (adapted from STAXI-2 manual $)^{2}$

Table 9. Baseline demographic data

Table 10. Baseline psychosocial data

Table 11. MBSR class attendance and homework completion

Table 12. Correlations between baseline trait anger, trait anger temperament, trait anger reaction, anger expression inwards, anger expression outwards, perceived stress and baseline 24-hour blood pressure

Table 13. Correlations between baseline trait anger, trait anger temperament, trait anger reaction, anger expression inwards, anger expression outwards, perceived stress and baseline daytime blood pressure 
Table 14. Correlations between baseline trait anger, trait anger temperament, trait anger reaction, anger expression inwards, anger expression outwards, perceived stress and baseline nighttime blood pressure

Table 15. Correlations between baseline anger control inwards, anger control outwards, 96 anger expression index and baseline 24-hour blood pressure

Table 16. Correlations between baseline anger control inwards, anger control outwards, 96 anger expression index and baseline daytime blood pressure

Table 17. Correlations between baseline anger control inwards, anger control outwards, 96 anger expression index and baseline nighttime blood pressure

Table 18. Changes in trait anger, trait anger temperament, trait anger reaction, anger expressed inwards, anger expressed outwards, perceived stress, anger control inwards, anger control outwards and the anger expression index from pre to post intervention

Table 19. Changes in the proportion of those scoring greater than or equal to the $75^{\text {th }}$ percentile in trait anger, trait anger temperament, trait anger reaction, anger expressed inwards, anger expressed outwards, anger control inwards, anger control outwards and anger expression from pre to post intervention

Table 20. Internal consistency measures using Cronbach's $\alpha$ at baseline and post intervention for trait anger, trait anger temperament, trait anger reaction, anger expression inwards, anger expression outwards, perceived stress, anger control inwards and anger control outwards

Table 21. Changes in 24-hour, daytime and nighttime blood pressure from pre to post 100 intervention

Table 22. Correlations between changes in trait anger, trait anger temperament, trait anger reaction, anger expression inwards, anger expression outwards, perceived stress and change in 24-hour blood pressure from pre to post intervention

Table 23. Correlations between changes in trait anger, trait anger temperament, trait 
anger reaction, anger expression inwards, anger expression outwards, perceived stress and change in daytime blood pressure from pre to post intervention

Table 24. Correlations between changes in trait anger, trait anger temperament, trait anger reaction, anger expression inwards, anger expression outwards, perceived stress and change in nighttime blood pressure from pre to post intervention

Table 25. Correlations between changes in anger control inwards, anger control outwards, anger expression index and change in 24-hour blood pressure from pre to post intervention

Table 26. Correlations between changes in anger control inwards, anger control outwards, anger expression index and change in daytime blood pressure from pre to post intervention

Table 27. Correlations between changes in anger control inwards, anger control outwards, anger expression index and change in nighttime blood pressure from pre to post intervention

Table 28. Effects of post intervention trait anger on post intervention 24-hour blood pressure after adjustment for baseline blood pressure, baseline psychosocial functioning and other covariates

Table 29. Effects of post intervention trait anger on post intervention daytime blood pressure after adjustment for baseline blood pressure, baseline psychosocial functioning and other covariates

Table 30. Effects of post intervention trait anger temperament on post intervention 24hour blood pressure after adjustment for baseline blood pressure, baseline psychosocial functioning and other covariates

Table 31. Effects of post intervention trait anger temperament on post intervention daytime blood pressure after adjustment for baseline blood pressure, baseline psychosocial functioning and other covariates 
Table 32. Effects of post intervention trait anger temperament as a multilevel categorical predictor variable divided into quartiles on post intervention 24-hour blood pressure after adjustment for baseline blood pressure, baseline psychosocial functioning and other covariates

Table 33. Effects of post intervention trait anger temperament as a multilevel categorical predictor variable divided into quartiles on post intervention daytime blood pressure after adjustment for baseline blood pressure, baseline psychosocial functioning and other covariates

Table 34. Effects of post intervention trait anger reaction on post intervention 24-hour 108 blood pressure after adjustment for baseline blood pressure, baseline psychosocial functioning and other covariates

Table 35. Effects of post intervention trait anger reaction on post intervention daytime 108 blood pressure after adjustment for baseline blood pressure, baseline psychosocial functioning and other covariates

Table 36. Effects of post intervention anger expressed inward on post intervention 24109 hour blood pressure after adjustment for baseline blood pressure, baseline psychosocial functioning and other covariates

Table 37. Effects of post intervention anger expressed inward on post intervention daytime blood pressure after adjustment for baseline blood pressure, baseline psychosocial functioning and other covariates

Table 38. Effects of post intervention anger expressed outward on post intervention 24hour blood pressure after adjustment for baseline blood pressure, baseline psychosocial functioning and other covariates

Table 39. Effects of post intervention anger expressed outward on post intervention daytime blood pressure after adjustment for baseline blood pressure, baseline psychosocial functioning and other covariates 
Table 40. Effects of post intervention perceived stress on post intervention 24-hour

blood pressure after adjustment for baseline blood pressure, baseline psychosocial functioning and other covariates

Table 41. Effects of post intervention perceived stress on post intervention daytime blood pressure after adjustment for baseline blood pressure, baseline psychosocial functioning and other covariates

Table 42. Effects of post intervention trait anger on post intervention nighttime blood pressure after adjustment for baseline blood pressure, baseline psychosocial functioning and other covariates

Table 43. Effects of post intervention trait anger temperament on post intervention nighttime blood pressure after adjustment for baseline blood pressure, baseline psychosocial functioning and other covariates

Table 44. Effects of post intervention trait anger temperament as a multilevel categorical predictor variable divided into quartiles on post intervention nighttime blood pressure after adjustment for baseline blood pressure, baseline psychosocial functioning and other covariates

Table 45. Effects of post intervention trait anger reaction on post intervention nighttime blood pressure after adjustment for baseline blood pressure, baseline psychosocial functioning and other covariates

Table 46. Effects of post intervention anger expressed inward on post intervention nighttime blood pressure after adjustment for baseline blood pressure, baseline psychosocial functioning and other covariates

Table 47. Effects of post intervention anger expressed outward on post intervention nighttime blood pressure after adjustment for baseline blood pressure, baseline psychosocial functioning and other covariates

Table 48. Effects of post intervention perceived stress on post intervention nighttime 
blood pressure after adjustment for baseline blood pressure, baseline psychosocial functioning and other covariates

Table 49. Effects of post intervention anger control inward on post intervention 24-hour 116 blood pressure after adjustment for baseline blood pressure, baseline psychosocial functioning and other covariates

Table 50. Effects of post intervention anger control inward on post intervention daytime 116 blood pressure after adjustment for baseline blood pressure, baseline psychosocial functioning and other covariates

Table 51. Effects of post intervention anger control inward on post intervention nighttime blood pressure after adjustment for baseline blood pressure, baseline psychosocial functioning and other covariates

Table 52. Effects of post intervention anger control outward on post intervention 24hour blood pressure after adjustment for baseline blood pressure, baseline psychosocial functioning and other covariates

Table 53. Effects of post intervention anger control outward on post intervention daytime blood pressure after adjustment for baseline blood pressure, baseline psychosocial functioning and other covariates

Table 54. Effects of post intervention anger control outward on post intervention nighttime blood pressure after adjustment for baseline blood pressure, baseline psychosocial functioning and other covariates

Table 55. Effects of post intervention anger expression index on post intervention 24hour blood pressure after adjustment for baseline blood pressure, baseline psychosocial functioning and other covariates

Table 56. Effects of post intervention anger expression index on post intervention daytime blood pressure after adjustment for baseline blood pressure, baseline psychosocial functioning and other covariates 
Table 57. Effects of post intervention anger expression index on post intervention nighttime blood pressure after adjustment for baseline blood pressure, baseline psychosocial functioning and other covariates

Table 58. Standardized and unstandardized effects of post intervention primary predictor variables on post intervention 24-hour blood pressure for all subjects after adjustment for baseline blood pressure, baseline psychosocial functioning and other covariates

Table 59. Standardized and unstandardized effects of post intervention primary predictor variables on post intervention daytime blood pressure for all subjects after adjustment for baseline blood pressure, baseline psychosocial functioning and other covariates

Table 60. Baseline demographic data by sex

Table 61. Baseline psychosocial data by sex

Table 62. Primary results summary: associations between primary post intervention 126 anger measures and post intervention 24-hour blood pressure after adjustment for baseline blood pressure, baseline psychosocial functioning and other covariates Table 63. Primary results summary: associations between primary post intervention anger measures and post intervention daytime blood pressure after adjustment for baseline blood pressure, baseline psychosocial functioning and other covariate

Table 64. Secondary results summary: associations between post intervention primary anger measures and post intervention nighttime blood pressure after adjustment for baseline blood pressure, baseline psychosocial functioning and other covariates

Table 65. Exploratory results summary: associations between post intervention exploratory anger measures and post intervention 24-hour blood pressure after adjustment for baseline blood pressure, baseline psychosocial functioning and other covariates 
Table 66. Exploratory results summary: associations between post intervention exploratory anger measures and post intervention daytime blood pressure after adjustment for baseline blood pressure, baseline psychosocial functioning and other covariates

Table 67. Exploratory results summary: associations between post intervention exploratory anger measures and post intervention nighttime blood pressure after adjustment for baseline blood pressure, baseline psychosocial functioning and other covariates

Table 68. Standardized and unstandardized effects of post intervention trait anger temperament on post intervention 24-hour systolic blood pressure for female subjects after adjustment for baseline blood pressure, baseline psychosocial functioning and other covariates

Table 69. Standardized and unstandardized effects of post intervention perceived stress on post intervention systolic blood pressure for female subjects after adjustment for baseline blood pressure, baseline psychosocial functioning and other covariates

Table 70. Standardized and unstandardized effects of post intervention perceived stress on post intervention systolic blood pressure for male subjects after adjustment for baseline blood pressure, baseline psychosocial functioning and other covariates 


\section{List of Figures}

Figure number. Description.

Page

Figure 1. Scatter plot of post intervention 24-hour systolic blood pressure correlated with post intervention perceived stress scale score for female participants. Legend: $A=$ 1 observation, $\mathrm{B}=2$ observations

Figure 2. Scatter plot of post intervention nighttime systolic blood pressure correlated with post intervention perceived stress scale score for female participants. Legend: $\mathrm{A}=$ 1 observation, $\mathrm{B}=2$ observations 


\section{Chapter 1 \\ Introduction}

\section{Hypertension: A Global Pandemic}

In 1961, the Framingham Heart Study identified elevated blood pressure as a risk factor for heart disease. ${ }^{3}$ Today, hypertension is recognized as one of the major risk factors for cardiovascular disease and death. ${ }^{4,5}$ This view has been supported by the World Health Organization which has stated that preventing and controlling hypertension is "one of the single most cost effective strategies for reducing the global burden of premature cardiovascular disease and death"., 6 Hypertension affects approximately $25 \%$ of Canadian adults aged 35-64 years ${ }^{7}$ and over $50 \%$ of people aged 65 years and older. ${ }^{8,9}$ Based on globally collected data, the number of adults with hypertension is estimated to increase by 60 percent by $2025 .{ }^{10}$ Taken together, this information outlines the significant contribution made by hypertension to worldwide death and chronic disease and its growth into a global pandemic.

\subsection{Risk Factors for Hypertension}

The origin of primary hypertension remains unknown. To date, the development of hypertension is best understood as the by-product of an accumulation of environmental and genetic risk factors. ${ }^{11}$ These traditional risk factors include diabetes, age, sex, excessive sodium consumption, lack of exercise and genetic background. ${ }^{12}$ Together, these factors are understood to increase the risk for blood pressure elevation and hypertension development. ${ }^{12}$

Psychosocial variables have also been linked to blood pressure as suspected risk factors implicated in the development of hypertension. ${ }^{13-16}$ The recognition of a relationship between psychosocial factors and blood pressure is reflected in the Canadian blood pressure guidelines. In 2007, the Canadian Hypertension Education Program (CHEP) - an organization focused on delivering evidence-based blood pressure guidelines for clinicians across Canada - adopted stress management as a lifestyle recommendation for hypertensive patients in whom stress appears to be an issue. ${ }^{17,18}$ The assimilation of stress management into national blood pressure guidelines indicates a growing acceptance of psychosocial factors as possible contributors to hypertension development. 
The consideration of psychosocial variables has also been recognized in the cardiovascular disease literature. The 2004 INTERHEART case-control study, carried out in 52 countries in over 29,000 patients, revealed that psychosocial stress was associated with an increased risk for myocardial infarction among all sexes, ages and regions of the world. ${ }^{19}$ The following year Rozanki et al. published a "State-of-the-Art Paper" in the Journal of the American College of Cardiology where the focus of the manuscript was to persuade clinicians to consider psychosocial risk factors in cardiac practice. ${ }^{20}$ Currently, the Heart and Stroke Foundation cites stress as a modifiable risk factor for heart disease ${ }^{21}$ and the Canadian Psychological Association cites psychosocial risk factors for heart disease. ${ }^{22}$ Taken together, this evidence outlines the growing acceptance of psychosocial variables as modifiable risk factors not only for hypertension but also cardiovascular disease.

\subsection{Pathophysiological Evidence \& Hypothesized Casual Pathways - Linking Psychosocial Variables \& Blood Pressure}

Several lines of evidence outline possible pathways by which psychosocial risk factors could affect blood pressure. Mechanisms underlying those relationships have been divided into 2 categories: pathophysiological mechanisms such as the over-stimulation of neuroendocrine pathways $^{23}$ and behavioural mechanisms whereby psychosocial variables increase the risk of engaging in poor health choices. ${ }^{24}$

One hypothesized pathway linking emotional arousal and blood pressure includes the overactivation of the sympathetic nervous system (SNS) and hypothalamic-pituitary-adrenal (HPA) axis. More specifically, that repeated stimulation of the cardiovascular system from SNS/HPA neurohormones due to psychosocial stress contributes to the progression and development of hypertension. Elevations in neurohormones have been demonstrated in response to acute $e^{25,26}$ and chronic stress, ${ }^{27-29}$ and evidence from the animal literature has demonstrated that long-term neurohormone infusion can produce sustained hypertensive states. ${ }^{30}$ Taken together, this information outlines how chronic emotional arousal could be linked to the development of hypertension via the over-stimulation of neuroendocrine pathways.

Research on cardiovascular reactivity has also provided a possible pathway connecting psychosocial stress and blood pressure. Cardiovascular reactivity is most frequently used as a 
laboratory tool to measure physiological stress responses to psychological stressors. Blood pressure is a measureable component of cardiovascular reactivity. Evidence has linked specific psychosocial variables such as anger to greater cardiovascular reactivity. ${ }^{31-35}$ Increased cardiovascular reactivity has in turn been associated with long-term increases in blood pressure ${ }^{36}$ and increased cardiovascular risk status. ${ }^{37}$ Together this information outlines an additional pathophysiological pathway by which chronic exposure to psychosocial stressors could influence the development of hypertension by altering cardiovascular reactivity profiles.

Psychosocial variables have also been linked to blood pressure via baroreflex pathways. Various types of psychosocial stress have been associated with reduced baroreflex sensitivity, including anxiety $^{38}$ and post-traumatic stress disorder (PTSD). ${ }^{39}$ Reduced baroreflex sensitivity has been associated with reduced parasympathetic and increased sympathetic activity. ${ }^{38,40}$ This altered baroreflex profile has been associated with increased blood pressure, even upon follow-up five years later. ${ }^{41}$ Anxiety and PTSD are very different illnesses; however they both share the commonality of a chronically stressed psychological state. Thus, taken together, this evidence outlines another possible mechanistic pathway by which chronic psychosocial stressors may be linked to elevated blood pressure through altering baroreflex sensitivity.

More recently, the immune system and inflammatory molecules have been implicated in hypertension development. ${ }^{42-45}$ Specific immunological features have been identified, such as "serum immunoglobulin [and] alterations in both humoral and cellular immune functions" 42 that distinguish hypertensive patients from their normotensive counterparts. Interestingly, chronic psychosocial stress has been reported to have negative effects on the immune system, ${ }^{46,47}$ and recent research has also documented psychosocial stressors such as perceived stress to co-vary with specific immunological markers like proinflammatory chemokine interleukin $8 .{ }^{48}$ The linkages between these variables outline another possible mechanism whereby psychosocial stressors could contribute to chronic diseases of inflammation via the immune system. ${ }^{49}$ Although evidence from this field is largely preliminary, it nonetheless outlines another pathway by which psychosocial variables may exert an effect on blood pressure.

Several pathways have been hypothesized that link psychosocial variables to elevated blood pressure and the development of hypertension. They include: the over-stimulation of neuroendocrine pathways, increased cardiovascular reactivity, reduced baroreflex sensitivity and 
altered immunological profiles. Evidence from this field of research has helped to strengthen the legitimacy of psychosocial variables as possible risk factors for hypertension development.

\subsection{Anger \& Perceived Stress as Psychosocial Risk Factors}

Anger $^{24,50-77}$ and perceived stress ${ }^{78-90}$ have been associated with higher blood pressure and poor health outcomes. Most evidence has been limited to observational research. A better understanding of anger and perceived stress as suspected risk factors for hypertension development is necessary to manage the burden of hypertensive disease. Prospective longitudinal cohort and experimental studies, specifically those that are designed to manipulate anger and perceived stress, would enable a more rigorous evaluation of these relationships. Current evidence on the relationship between anger, perceived stress and blood pressure, as well as the use of therapeutic psychological interventions to design a prospective longitudinal cohort study that manipulates anger and perceived stress will be discussed.

\section{Anger \& Blood Pressure}

\subsection{Historical Context \& Definitions}

Anger is recognized as both an emotional state and a personality type. Most commonly, anger is thought of as "feelings that vary in intensity from mild irritation or annoyance to intense fury and rage". 2 As a personality type, anger refers to the propensity for some individuals to experience angry feelings more frequently than others. Anger is often mistakenly used interchangeably with other behaviours or personalities such as hostility and aggression. Much of this misuse is due to the contribution of anger to the characterization of these other behaviours and personalities. For instance, hostility involves the "frequent experience of angry feelings, but also has the connotation of a complex set of attitudes that includes meanness and viciousness, as well as aggressive and often vindictive behaviour". ${ }^{2}$ For this reason, anger is "generally considered to be a more fundamental concept than hostility or aggression [as] anger refers to feelings [while the latter]... describe [a collection of] negative attitudes and destructive or punitive behaviour". ${ }^{2}$ Thus, anger is both an emotion and a personality type that maintains its own construct and definition separate from other related psychosocial concepts.

A relationship between anger and blood pressure was first proposed in 1939 when Franz Alexander published the concept of the "hydraulic model". 91 Suppressed anger was hypothesized 
to have a maladaptive effect on blood pressure while expressed anger was thought to have the opposite. This distinction between suppressed and expressed anger was further developed by Funkenstein et al. in $1954 .^{92}$ Despite these early beginnings, research on the effects of anger on the cardiovascular system did not begin in earnest until the 1980s when the role of anger as the central component of Type A behaviour syndrome was identified. ${ }^{93}$ Two teams run by Ernest Harburg and Charles D. Spielberger led the research on studying anger's contribution to the etiology and progression of medical disorders. In a long-term hypertension research project, Harburg and colleagues investigated the relationships between anger expression and blood pressure using responses to hypothetically anger-proving questionnaires. ${ }^{52,53,94-96}$ Preliminary results linked anger expression to blood pressure. These findings were combined with cardiovascular reactivity literature on blood pressure and peripheral resistance, ${ }^{97}$ together developing the idea of a "hypertensive personality". 98,99 These combined efforts built the framework necessary to push research on the psychobiological effects of anger to a new level.

Attempts to explain these new relationships between anger and blood pressure emerged. Early psychoanalytic theories from Alexander's time proposed anger expression inward to cause feelings of guilt and depression which somehow contributed to negative physiological consequences. ${ }^{2,100}$ Later theories attempted to explain findings on a more basic pathophysiological level. These included linking anger expression to over-activation of the SNS and HPA axis where repeated increases in heart rate, blood pressure, vascular resistance and secretion of cortisol and catecholamines were hypothesized to initiate a hypertensive cascade. ${ }^{101,}$ 102 The growth and expansion of pathophysiological research demonstrated the increasing consideration of anger as a possible contributor to the development of hypertension.

As research progressed, calls began to emerge for more conceptual distinctions of anger to more accurately assess anger's contribution to different diseases. Spielberger was the most notable contributor to this movement, ultimately developing the validated State-Trait Anger Expression Inventory (STAXI). ${ }^{103,104}$ The questionnaire was novel for 2 reasons: 1 ) it further differentiated anger from hostility and aggression; and 2) it identified and measured additional and distinct anger-related constructs, separate from anger expression. Anger expression was also further expanded upon by the new questionnaire. This collective work from both Harburg and Spielberger developed anger into two separate but related concepts: anger experience and anger expression. 
Anger experience addressed the physical experience of anger, asking: how often and with what intensity did individuals experience angry feelings? In order to measure this, anger experience was differentiated into "state" and "trait" anger. State anger was defined as a "psychobiological emotional state or condition marked by subjective feelings that var[ied] in intensity from mild irritation or annoyance to intense fury and rage"2 accompanied by physiological responses from both neuroendocrine and autonomic nervous systems. State anger was further divided into subscales: feeling angry, feeling like expressing anger verbally and feeling like expressing anger physically. As opposed to angry feelings at a particular moment in time, trait anger measured "individual differences in the disposition to perceive a wide range of situations as annoying or frustrating and by the tendency to respond to such situations with elevations in state anger". ${ }^{2}$ It was expected that "individuals with high trait anger scores [would] experience state anger more often and with greater intensity than individuals who [were] low in trait anger". 2 Trait anger was also divided into subscales: anger temperament (which measured the disposition to experience anger without specific provocation) and anger reaction (which measured the frequency that angry feelings were experienced in situations that involved frustration and/or negative evaluations). Thus, according to the developments by Spielberger, state anger measured the level of angry feelings at a specific moment of testing whereas trait anger measured how often angry feelings were experienced over time.

Anger expression as defined by Spielberger was differentiated into 2 different subtypes: 1) anger expression outward, which involved the expression of anger toward other persons or objects in the environment; and 2) anger expression inward, which represented anger directed inside such as holding in or suppressing angry feelings. ${ }^{2}$ Spielberger also included the concept of "anger control" in the category of anger expression, viewing the concept of anger expression on a continuum with the control of anger as one endpoint and the expression of anger as the other. Anger control was similarly categorized: anger control outward measured the control of angry feelings by preventing the expression of anger toward other persons or objects in the environment, and anger control inward measured the control of suppressed angry feelings by calming down or cooling off. ${ }^{2}$ As part of addressing the existence of anger expression on a continuum, Spielberger also developed an overall omnibus anger expression measure titled the “anger expression index". The anger expression index provided a total measure of anger 
expression, based on how anger was expressed (inward or outward) and how anger was controlled (inward or outward). Combined, these scales defined anger expression.

Evidence supporting the validity of this framework for understanding anger was demonstrated by the widespread uptake of anger as a valid measureable construct. ${ }^{105,106}$ The STAXI itself was translated into different languages, ${ }^{107,108}$ further reinforcing the validity of its theoretical framework. No other validated anger questionnaire has contributed as substantially to our understanding of anger and its psychobiological effects. The combination of groundwork laid by Harburg, improved conceptualization of anger by Spielberger and development and widespread uptake of the STAXI questionnaire together represent some of the most important contributions to the field of anger research.

Thus, to summarize, anger is both an emotional state and a personality type that is distinct from other anger-like constructs such as hostility and aggression. Research studying the relationship between anger, blood pressure and cardiovascular disease began in 1939 with Franz Alexander and has evolved significantly since that time. Evidence from this research on the psychobiological relationship between anger, blood pressure and cardiovascular disease has significantly shaped the conceptualization of anger from both a psychological and physiological perspective. Teams led by Harburg and Spielberger have contributed significantly to our understanding of anger. Most notable has been the development of the validated STAXI questionnaire which has enabled a better understanding of anger and its relationship to different diseases.

\subsection{Evidence to Date on Anger \& Blood Pressure}

The contribution of anger to the development of hypertension has been studied extensively. Since the proposition of Alexander's original hydraulic model, where anger expressed inward was associated with increased blood pressure and anger expressed outwards was associated with decreased blood pressure, to date approximately half of the accumulated literature has reported some association between these 2 variables. ${ }^{109}$

However, interpreting this literature has been complicated by several factors including different outcome measures between studies (i.e., examining associations between anger and blood pressure as continuous variables vs. dichotomous or categorical variables), a lack of prospective, 
rigorously controlled longitudinal cohort or experimental research that measures these variables at more than one point in time, a lack of consideration for sex/gender or race-ethnicity and a lack of consistent pathophysiological mechanistic explanations. Evidence both supporting and refuting a relationship between anger and blood pressure will be presented below.

The majority of anger and blood pressure research has used the STAXI questionnaire. As the STAXI measures distinct anger constructs (state anger, trait anger, anger expression inward, anger expression outward, anger control inward and anger control outward) many of these constructs have individually been investigated for association with blood pressure. Therefore, the following review of the literature will address each anger construct and its relationship to blood pressure individually.

\subsubsection{State Anger}

State anger (the experience of angry feelings and associated physiological response at the moment of testing) is, at best, weakly associated with blood pressure. In total, 5 cross-sectional studies have examined this relationship. Four of these cross-sectional studies examined state anger by comparing the following groups: hypertensives and normotensives; ${ }^{66}$ mild hypertensives and normotensives; ${ }^{99}$ medicated hypertensives, unmedicated hypertensives and normotensives, ${ }^{110}$ and hypertensives and white coat hypertensives. ${ }^{58}$ One cross-sectional study examined associations between state anger and blood pressure as continuous variables. ${ }^{57}$ From all 5 studies, only 1 reported a significant difference in state anger between hypertensives and normotensives. ${ }^{66}$ No state anger subscales have been investigated for association with blood pressure. See Table 1 for a summary.

The state anger studies were roughly evenly distributed in terms of male and female subject representation, with a slight preference for male subjects. All studies administered the STAXI and all but 1 reported the use of a reliable method of blood pressure measurement ${ }^{66}$ (where reliable blood pressure measurement refers to either automated office, automated home, ambulatory or standardized manual blood pressure measurement). Interestingly, the only study that used unreliable blood pressure measurement techniques was the only study to report significant findings. ${ }^{66}$ 
Based on the abovementioned evidence there appears to be, at best, a weak signal between state anger and blood pressure. As mentioned, only 1 study reported a significant finding. This study also implemented the least rigorous blood pressure measurement techniques therefore making their findings the least robust. In addition to weak methodology, a second concern was the inclusion of war veterans as the hypertensive sample under study. The inclusion of such participants raises questions about the impact of war experiences on anger and thus the generalizability and applicability of the results. Thus, while a relationship between state anger and blood pressure may exist, based on the appraisal of the available evidence this appears unlikely.

Part of the struggle in identifying a relationship between state anger and blood pressure may lie in the fundamental construct of state anger itself. State anger addresses how angry an individual feels during that particular moment of testing. Thus, an ideal study would test for associations between state anger and blood pressure when individuals are in an angry state. Only 1 study attempted this approach and reported negative results. ${ }^{57}$ The paucity of such research exists for several reasons; most obvious are the logistical challenges of designing such a study. One approach could involve a case-control design where levels of state anger and 24-hour ambulatory blood pressure are compared between subjects suffering from anger-related issues and non-angry controls. Based on the current status of the state anger literature, confirming any associations between state anger and blood pressure will likely be a slow process.

The above-mentioned results are fairly consistent. State anger appears, at best, weakly associated with blood pressure. If future research is contemplated it should use more rigorous measurement tools and strive to examine associations between state anger and blood pressure when subjects are in an angry state.

\subsubsection{Trait Anger}

Trait anger measures how often angry feelings are experienced over time. Compared to state anger, trait anger innately assumes a more constant role in individuals' lives. This difference has been reflected in the literature by the increased number of studies examining trait anger and blood pressure. Reports from the literature suggest an association between trait anger and blood pressure; however, this relationship is potentially moderated in part by sex/gender and race/ethnicity. 
To date, 9 cross-sectional, 2 longitudinal and 1 interventional study have examined trait anger and blood pressure. ${ }^{57,58,66-69,72-75,99,110}$ See Table 2 for a summary. All studies used the STAXI and all but 3 reported a reliable method of blood pressure measurement. ${ }^{66,67,74}$ Thus, it can be concluded that most studies were methodologically sound with respect to measurement techniques. Trait anger subscales and their relationship to blood pressure have only been evaluated in 1 study that examined war veterans. ${ }^{66}$ Due to the possible impact of war experiences on anger and the greater amount of trait anger research this study will not be considered in this section.

\section{Trait Anger: Cross-Sectional Evidence}

Beginning with cross-sectional evidence reporting no association between trait anger and blood pressure, 4 out of 9 cross-sectional studies fall in this category. Three of these studies examined trait anger by comparing the following groups: mild hypertensive and normotensive adults (all male and majority Caucasian); ${ }^{99}$ medicated hypertensive, unmedicated hypertensive and normotensive adults (all female and African American); ${ }^{110}$ and hypertensive and white-coat hypertensive university students (majority male and Caucasian). ${ }^{58}$ The remaining cross-sectional study reporting null results examined associations between trait anger and blood pressure as continuous variables among African American and Caucasian male and female high schoolstudents. ${ }^{57}$ The 5 remaining cross-sectional studies that did report significant findings similarly examined differences in trait anger or blood pressure between specific groups or associations between trait anger and blood pressure as continuous variables. All 5 studies reporting positive results included normotensive subjects along with either white-coat hypertensives, ${ }^{72}$ mild hypertensives ${ }^{68,73}$ or hypertensives. ${ }^{66,67}$ Together, this information outlines the diversity and significant number of studies that have examined relations between trait anger and blood pressure.

Comparing the positive and negative studies revealed an interesting pattern. All 5 positive studies included at least $40 \%$ female participants or more (with the exception of the excluded male war veteran study). ${ }^{66}$ This contrasted with the 4 negative studies which were all male, ${ }^{58,99}$ a combination of males and females ${ }^{57}$ or only females. ${ }^{110}$ Closer inspections of the negative studies that did include female subjects revealed specific factors that may have explained their null results. A critical appraisal of these factors will be discussed in the following section. In the 
interim, it seems possible that trait anger is moderated in part by sex - or, more specifically, that a signal may only be observed among females. Most studies did not re-analyze their data by sex; therefore, it is not possible to verify this observation. However, this pattern does outline the possible existence of a sex effect and an explanation for the trend observed in the literature.

As stated above, an appraisal of the negative studies that included female subjects revealed specific factors that could explain the null findings. The first factor was age. One of the 2 negative studies recruited male and female high school students (aged 15-17 years). ${ }^{57}$ Hypertension is more prevalent among older individuals. ${ }^{12}$ Therefore, the generalizability of data from high school students is questionable. The second factor was choice of outcome measure; more specifically, the potential to obtain different results when studying differences between groups rather than associations between variables. The second negative study reported no difference in trait anger between medicated hypertensive, unmedicated hypertensive and normotensive middle-aged females. ${ }^{110}$ While the authors reported no difference between groups, they did not demonstrate that trait anger was not associated with blood pressure. By comparison, a positive cross sectional study by Durel et al. did report significant findings among females when trait anger was analyzed for association with blood pressure. ${ }^{73}$ Thus, it is possible that results from the second neutral study (despite its inclusion of female subjects) could be accounted for by choice of outcome measure. Together, this information highlights specific study features that may explain why the 2 negative studies that included female subjects contrast with the overall observed sex-effect pattern.

In summary, more cross-sectional research has reported positive associations between trait anger and blood pressure ( 5 positive studies vs. 4 negative studies). This relationship may be moderated in part by sex/gender whereby the effect of trait anger on blood pressure is restricted to females.

\section{Trait Anger: Longitudinal Evidence}

The cross-sectional research studying the linkage between blood pressure and trait anger has been followed up by longitudinal research. In 1991, Markovitz et al. conducted a prospective 3year longitudinal follow-up of normotensive middle-aged females (7\% identifying as nonCaucasian). ${ }^{69}$ The authors found that increases in trait anger were independently associated with increases in systolic and diastolic blood pressure over three years of follow-up. In 2007, as part 
of secondary analyses from the Atherosclerosis Risk in Communities (ARIC) study, Player et al. explored the influence of trait anger on the progression of prehypertension to hypertension. ${ }^{74}$ The authors followed a cohort of middle-aged males and females for 4-8 years (20\% identifying as African American, 80\% not identifying as African American). High levels of trait anger were associated with a progression to hypertension. However, after stratifying by sex, trait anger was predictive for males only.

Again, while at first contradictory, discrepant findings between these 2 studies may be attributed to different outcome measures and sample populations under study. Markovitz et al. studied blood pressure change over time among normotensive females who almost all self-identified as Caucasian. No comparator group existed for male or African American subjects in this setting. Player et al. studied males and females but specifically changes in hypertensive status from prehypertension to hypertension rather than a change in blood pressure. One explanation for these discrepant findings could include that trait anger contributes to blood pressure change over time, but that this change in blood pressure does not have the same effect in changing hypertensive status between males and females. It is well established that sex has an important impact on blood pressure. Until menopause ${ }^{111}$ females demonstrate lower blood pressure than males. ${ }^{112-114}$ Findings from Player et al. may be a reflection of prevalence trends, where a greater proportion of males become hypertensive at middle age compared to age matched females.

In summary, the longitudinal evidence suggests that trait anger has some impact on blood pressure over time. This evidence also reinforces sex/gender and race/ethnicity as possible mediators of that relationship.

\section{Trait Anger: Experimental/Interventional Evidence}

One experimental study has added more clarity to the literature. ${ }^{75}$ In a simulated call center study, female subjects were instructed to react to a complaining customer "either authentically and show their true emotions" or "serve with a smile and hide negative emotions". Race/ethnic background was not specified. Using the STAXI and automated blood pressure measurement, the authors found that trait anger moderated effects on diastolic blood pressure in the "serve with a smile" scenario. Higher trait anger was also associated with higher diastolic blood pressure. This experimental evidence further supports the possible existence of a relationship between trait 
anger and blood pressure and reinforces the cross-sectional and longitudinal evidence reporting a relationship between these variables among females.

\section{Trait Anger: Summary}

Based on the cross-sectional, longitudinal and experimental research, trait anger appears to have some effect on blood pressure, possibly affecting females more than males. However, some of the mixed-sex cross-sectional research and longitudinal evidence would suggest that trait anger affects males as well. Racial/ethnic contributions are still unclear and could still play significant mediating roles.

\subsubsection{Anger Control}

Anger control has been less studied, perhaps due in part to its more complicated theoretical construct. Two research teams that examined the relationship between anger control and blood pressure produced conflicting results. ${ }^{24,115}$ Igna et al. studied 705 middle-aged males in a trial focused on the prevention of metabolic syndrome, type-2 diabetes and cardiovascular disease. They found that anger control was significantly positively associated with blood pressure. By contrast, Ohira et al. who studied 790 male Japanese nuclear power plant workers determined that anger control was not associated with blood pressure. Both studies used the STAXI as well as standardized manual blood pressure measurement. See Table 3 for a summary.

Based on the limited number of conflicting studies it is not possible to make meaningful conclusions. Future research remains to be completed on the relationship between anger control and hypertension. If contemplated, it should investigate the existence of sex differences and follow participants longitudinally to clarify these authors' findings.

\subsubsection{Anger Expression}

Anger expression is the most frequently researched anger construct with respect to effects on blood pressure. To review, anger expression has been separated into 2 types: anger expressed in (which refers to anger directed inward such as holding in or suppressing angry feelings) and anger expressed out (which refers to the expression of anger toward other persons or objects in the environment). ${ }^{2}$ For the purposes of this study, suppressed anger will always be referred to as anger expressed in and expressed anger will always be referred to as anger expressed out. The 
term "anger expression" does not specify which type of expression is being discussed and thus will refer to the broad category encompassing both anger expressed in and anger expressed out.

In general, the literature has reported either a positive association or no association between anger expressed in and blood pressure, and more mixed associations between anger expressed out and blood pressure (i.e., positive, negative and no association). Unfortunately, summarizing the anger expression literature has been complicated by methodological inconsistencies between studies such as the use of differing anger assessment tools and outcome measures. Differing anger assessment tools are due in part to the concept of anger expression emerging in 1939 and the STAXI questionnaire emerging much later (1980s). During this time, anger was assessed using various instruments. The use of these different instruments has made comparisons between studies more difficult. Differing study outcome measures has also added complexity. As highlighted in the trait anger research, one study may evaluate associations between variables and another may dichotomize variables and study differences between groups. Although these approaches are asking the same fundamental question, they are giving different answers. Combining these methodological inconsistencies with the large number of studies has made synthesizing the anger expression literature much more complex (Table 4).

Explaining this state of the literature is necessary to outline the path taken in this review. Studies have first been separated by anger expression type (expressed in or expressed out). Following this, each type has been subdivided into studies reporting a negative relationship, positive relationship or no relationship. The type of blood pressure measurement tool, self-report anger questionnaire, details of the study subjects and outcome measure have been included where appropriate. A summary of this anger expression literature can be found in Table 5.

\subsubsection{Anger Expressed In}

To review, anger expressed inward addresses how often angry feelings are experienced but not expressed.

\section{Anger Expressed In: Negative Relationship}

To the best of our knowledge, no study has reported a negative relationship between anger expression inwards and blood pressure. 


\section{Anger Expressed In: Positive Relationship}

In 1973, Harburg et al. were among the first to report that anger expressed in, as measured by the Harburg questionnaires, was positively associated with standardized manual diastolic blood pressure. ${ }^{53}$ The average magnitude of difference between subjects who expressed anger inward versus expressed anger outward was $\pm 3.93 \mathrm{mmHg}$. Additionally, men who expressed anger inwards reported a greater prevalence of hypertension (19\%) compared to those who expressed anger outwards (12.25\%). Approximately 10 years later in a follow-up study, the same research team examined the effects of race, sex and anger expression style on blood pressure. ${ }^{52}$ On average, subjects reporting high anger expression inward had significantly higher diastolic blood pressure levels compared to subjects reporting medium or high levels of anger expression outward. Anger expressed in was also independently associated with an increased risk for being classified as hypertensive versus normotensive."

* Note of clarification: It is stated in the Harburg 1973 publication that "the use of Funkenstein, et al.'s terms of 'Anger In' and 'Anger Out' [was] solely for ease of labelling; we did not measure the biological responses of anger." However, 10 years later in Gentry et al.'s 1982 publication, the authors stated the following: "Elsewhere, Harburg et al. [authors are referring to Harburg et al. 1973 publication] have used the concept of 'suppressed hostility' to describe an individual's tendency to 1) inhibit aggressive attitudes or actions and 2) feel guilty if such behavior should be expressed. In this paper, we have chosen to use the term 'suppressed anger,' which (based on the [same] actual questionnaire items) describes the individual's tendency not to get annoyed, mad, or angry and which essentially identifies the emotional component of the frustration-aggression sequence". Based on this information, it has been understood that the authors were measuring anger expression in 1973. However, at that time it appears that it was categorized as an emotion under the broader construct of hostility. Thus, for the purposes of this literature review, Harburg et al. did look at anger expression in 1973 as it is understood at present day. 
Since the publication of the Harburg et al.'s findings, 7 cross-sectional studies have supported their reports. ${ }^{50,51,57,58,67,70,71}$ These consist of 3 studies where the primary analysis evaluated associations between anger and blood pressure as continuous variables ${ }^{51,57,71}$ and 4 studies where the primary analysis examined blood pressure or anger levels between groups. ${ }^{50,58,67,70}$ One longitudinal cohort study has also provided support. ${ }^{62}$ Using prospectively collected epidemiological data, the longitudinal study found that every 1-point increase in anger expressed in on the STAXI corresponded to a 12\% increased risk for hypertension after 4 years of followup among male subjects. The analysis used in this study was specified a priori thus reinforcing these authors' findings. Taken together, this cross-sectional and longitudinal evidence outlines the current support for a positive association between anger expressed inward and blood pressure.

The studies presented above are strictly those that have reported a positive relationship between anger expression inward and blood pressure. On an individual study basis, there are occasional differences with respect to sex/gender and race/ethnicity. However, the distribution of studies reporting significant results for female subjects ${ }^{70,71}$ is balanced by the number of studies reporting significant results for all male studies ${ }^{50}$ or majority male studies (i.e., $91 \%$ male subjects). ${ }^{58}$ Thus, underlying sex/gender and race/ethnic effects remain unclear.

\section{Anger Expressed In: No Relationship}

To date, 6 cross-sectional studies have reported no association between anger expressed inward and blood pressure. ${ }^{24,72,77,99,110,115}$ Three of these studies examined associations between blood pressure and levels of anger expression inward ${ }^{24,77,115}$ while 3 studied differences of anger expression inward or blood pressure between groups. ${ }^{72,99,110}$ All studies used the STAXI. Together these studies outline the cross-sectional evidence reporting no association between anger expressed inward and blood pressure.

There are also 2 longitudinal cohort studies that have reported no association between anger expressed in and blood pressure. ${ }^{61,69}$ Markovitz et al. conducted the prospective longitudinal follow-up of normotensive middle-aged women using the STAXI questionnaire and standardized manual blood pressure measurement. They reported that anger expression inward was not associated with blood pressure over the 3-year follow-up period. ${ }^{69}$ The second longitudinal study recruited male and female subjects, used the Harburg questionnaire and manual blood pressure 
measurement. ${ }^{61}$ These authors also found no association between anger expression inward and blood pressure over the 7-year follow-up period. Together, these 2 works outline the longitudinal evidence against an association between anger expressed in and blood pressure.

Although the above-mentioned longitudinal evidence offers more robust evidence against an association between anger expressed inward and blood pressure, it should be known that one author's (Julius et al.) decision to analyze psychosocial functioning and blood pressure was included as post-hoc analysis. ${ }^{61}$ Based on this detail, Julius et al.'s findings can only be interpreted as hypothesis generating. By contrast, Markovitz et al. specified their longitudinal analysis a priori. Thus, their findings can be considered valid evidence of no association between anger expressed inwards and blood pressure among female subjects. ${ }^{69}$ It is necessary to know this information in order to accurately evaluate the contribution of these studies to the literature.

\section{Anger Expressed In: Summary}

To summarize, 10 studies reported a positive relationship between anger expressed in and blood pressure while 8 studies reported no association. From the 10 studies that supported a positive relationship, 9 were cross-sectional and 1 was longitudinal. Among the 8 studies reporting no association, 6 studies were cross-sectional and 2 were longitudinal. Based on an appraisal of the analysis used in one of the no association longitudinal studies, it was determined that only 1 longitudinal study provided acceptable evidence against an association between anger expressed inward and blood pressure. ${ }^{69}$ Thus, there are only 7 valid studies reporting no relationship between anger expressed inward and blood pressure. Overall, there are more studies supporting a relationship between anger expressed inwards and blood pressure rather than refuting it.

Taking a collective approach to the cross-sectional research reveals that study details were relatively homogenous between those reporting positive associations versus no associations. Overall, the methodological quality, which addressed blood pressure measurement techniques and self-report questionnaires as well as appropriateness and generalizability of the sample population under study, was consistent. The proportion of male and female subjects was generally even, as were the number of studies recruiting Caucasian and non-Caucasian subjects. Finally, types of outcome measures were also evenly distributed (i.e., number of studies that evaluated associations between continuous variables vs. studies that examined anger or blood 
pressure levels between groups). In summary, methodological differences between studies reporting positive associations versus no associations were minimal.

An evaluation of the longitudinal evidence does not reveal the same consistency. Significant differences between the 3 longitudinal studies include the sex of sample populations under study and outcome measures. ${ }^{61,62,69}$ The low number of studies has likely amplified any underlying effects from confounding or biases; therefore, results must be interpreted accordingly. In summary, the longitudinal evidence has also produced conflicting findings. However, the degree to which these findings can be explained by the relationship between anger and blood pressure is constrained by methodological inconsistencies between studies.

To summarize, the literature on anger expression inward and blood pressure is largely divided between studies reporting a positive association and studies reporting no association. However, based on raw number of studies, there may be a stronger evidence for a positive relationship. This has been outlined by both cross-sectional and, to a lesser degree, longitudinal research.

\subsubsection{Anger Expressed Out}

To review, anger expression outward addresses how often angry feelings are expressed using verbally or physically aggressive behaviours. Guided under Alexander's original 1939 hypothesis - where anger expression outward was an adaptive behaviour that decreased blood pressure - anger expressed outward has traditionally been investigated for a negative association with blood pressure. However, as will be discussed, compared to anger expression inward, the evidence emerging from this field has been more varied.

\section{Anger Expressed Out: Negative Relationship}

Six cross-sectional studies support Alexander's original hypothesis. ${ }^{24,50,52,53,76,115}$ These 6 studies are methodologically fairly uniform. In their primary analyses, three studies evaluated associations between anger expression outward and blood pressure as continuous variables ${ }^{24,76}$, 115 while 3 dichotomized anger and examined association with blood pressure levels. ${ }^{50,52,53}$ All used reliable blood pressure measurement techniques. However, 4 studies did not use the STAXI, instead opting for either the Harburg Anger-In/Anger-Out Scale ${ }^{50,52,53}$ or the Behavioural Anger Response Questionnaire. ${ }^{76}$ Thus, their findings should be interpreted 
accordingly. In terms of racial distribution, there was a slight favour for Caucasian subjects. With respect to sex/gender, approximately half of the studies included only male subjects with the other half including at least 50\% males. Therefore, an underlying sex bias may exist. But overall these 6 cross-sectional studies are relatively similar.

The studies presented here are strictly those that have supported a negative association between anger expression outward and blood pressure. Besides the use of different anger assessment instruments, these studies are relatively homogenous. However, generalizability of results to females is questionable due to the low number of female participants studied.

\section{Anger Expressed Out: Positive Relationship}

Four studies have reported a positive association between anger expression outwards and blood pressure. This consists of 3 cross-sectional ${ }^{57,72,77}$ and 1 longitudinal study. ${ }^{62}$ All used the STAXI and reliable blood pressure measurement techniques. Outcomes included associations between anger and blood pressure ${ }^{57,77}$ as well as anger differences between hypertensive classification groups. ${ }^{72}$ The longitudinal study has provided the most robust evidence for a positive association; every 1-point increase in anger expressed outward corresponded to a $12 \%$ increased risk for hypertension after 4 years of follow-up. ${ }^{62}$ While racial/ethnic distributions were relatively even between studies, sex/gender was not. The 2 largest studies (one of which was the longitudinal cohort) included only middle-aged males; ${ }^{62,77}$ therefore, it is unclear if this relationship would be observed among females. This potential male bias is reinforced by one of the mixed-sex cross-sectional studies which only reported an association among Caucasian males. ${ }^{57}$ Taken together, these 4 studies suggest that anger expression outward is positively associated with blood pressure, possibly interacting with sex/gender and affecting males more than females. The lack of female participants limits the generalizability of results. Results should be interpreted accordingly.

\section{Anger Expressed Out: No Relationship}

Slightly more research has reported no relationship between anger expressed outward and blood pressure. This evidence consists of 7 studies: 2 longitudinal ${ }^{61,69}$ and 5 cross-sectional. ${ }^{58,67,70,71 \text {, }}$ 99 
From the cross-sectional research, 1 study examined blood pressure and anger scores as continuous variables $^{71}$ and 4 dichotomized anger expression outward or blood pressure and examined levels between groups. ${ }^{58,67,70,99}$ All but 1 study used the STAXI, ${ }^{71}$ all but 2 reported the use of reliable blood pressure measurement techniques ${ }^{67,71}$ and all but 2 included both sexes/genders ${ }^{99}\left(30 / 33\right.$ male $\left.^{58}\right)$. In terms of race/ethnicity, approximately three quarters of the participants studied were Caucasian. Given the generally consistent use of reliable blood pressure measurement techniques, validated anger questionnaires and inclusion of male and female study participants these studies outline some of the valid cross-sectional evidence reporting no association between anger expressed outward and blood pressure, at least among Caucasian subjects.

Once more, the evidence from the longitudinal research is less robust. ${ }^{61,69}$ This is attributed in part to the hypothesis generating findings from Julius et al. who examined anger and blood pressure through post-hoc analyses. ${ }^{61}$ Due to this arrangement, their findings cannot be considered in a conclusive context. The remaining longitudinal study (which did arrange its analysis a priori) reported no association between anger expressed outward and blood pressure among female participants (97\% Caucasian) after 3 years of follow-up. ${ }^{69}$ Although these results are more robust compared to cross sectional evidence, 1 longitudinal study on Caucasian females cannot determine the direction for the field.

\section{Anger Expressed Out: Summary}

To summarize, 6 studies reported a negative association between anger expressed out and blood pressure, 4 reported a positive association and 7 reported no association. The 6 studies reporting a negative association were all cross-sectional. Among the 4 studies reporting a positive association 3 were cross-sectional and 1 was longitudinal. Finally, among the 7 studies reporting no association, 5 were cross-sectional and 2 were longitudinal. Based on an appraisal of the analysis approach used in one of the no association longitudinal studies, it was determined that only 1 longitudinal study provided acceptable evidence against an association between anger expressed outward and blood pressure. ${ }^{69}$ Overall, the methodological quality, which refers to type of blood pressure measurement technique and self-report anger questionnaire as well as appropriateness and generalizability of sample population under study, was relatively consistent between groups (where 'groups' refers to studies reporting a negative association vs. a positive 
association vs. no association). Exceptions to this were the negative association and positive association studies that studied more males than females. Only among the 7 no association studies was there more equal representation of males and females. Taken together, this trend may outline an interaction with sex/gender whereby a relationship between anger expression outward and blood pressure (be it positive or negative) is only found among males. Conflicting findings between the positive relationship and negative relationship studies remain to be clarified. Thus, at this time, no clear direction for the field can be discerned.

\subsubsection{Synthesizing the Evidence}

Overall, anger (as measured by its respective subcomponents) may be associated with blood pressure. However, this is not consistent across each subcomponent. For some subcomponents, the evidence is inconclusive. A summary of each anger construct and its relationship to blood pressure is presented below.

\section{State Anger}

State anger appears to be, at best, weakly positively associated with blood pressure. Research examining associations between state anger and blood pressure when subjects are in an angry state is necessary to determine whether an association exists with blood pressure. From a longitudinal perspective, it would also be of interest to determine whether repeated exposure to (or greater) state anger is associated with a significant increase in blood pressure over time.

\section{Trait Anger}

Trait anger appears to be positively associated with blood pressure. However, this relationship may be moderated by other covariates such as sex/gender and race/ethnicity. Associations between these 2 variables cannot be confirmed until prospective longitudinal cohort and experimental studies, particularly those that manipulate anger, are conducted on both sexes.

\section{Anger Control}

Two studies examining the association between anger control and blood pressure reported conflicting results. Both studied male-only sample populations. Much more research is required to clarify these findings. 


\section{Anger Expression (inward and outward)}

The literature is almost evenly divided with respect to either a positive association or no association between anger expressed inward and blood pressure. The literature on anger expressed outward is also divided but between all relationship types: negative, positive or no association. While there are a greater number of no association studies in the anger expressed outward research ( 7 studies vs. 6 and 4 studies in the other categories), the uneven distribution of male and female subjects between the categories outline a potential sex-bias. Therefore, the available anger expression evidence, both inward and outward, is inconclusive with respect to either supporting or refuting a significant association with blood pressure.

\section{Literature Gaps}

Several literature gaps emerge when considering the collective effects of anger on blood pressure.

First is the limited or inconsistent consideration of race/ethnicity and gender/sex as covariates in many research models. It is understood that elevated blood pressure is more common among males than females until females reach menopause ${ }^{111}$ and that elevated blood pressure more is common among African American populations. ${ }^{116}$ There is also evidence suggesting that African Americans and Caucasians do not score the same on validated anger questionnaires; a finding that interacts with sex/gender on some occasions. ${ }^{54-57,117}$ Based on this information, the consideration of sex/gender and race/ethnicity is necessary to better understand the relationship between anger and blood pressure. This view has been shared by many others. ${ }^{73,99,118-123}$

Second is the use of non-validated anger questionnaires and reliable blood pressure measurement techniques. The use of unreliable tools raises the question of reliability of results. Moreover, poor or differing methodology makes comparison of results between studies very difficult. With respect to blood pressure, it has been reported that compared to standardized blood pressure assessments, non-standardized manual blood pressure measurement techniques can change the hypertension-normotension classification of blood pressure in over $50 \%$ of persons. ${ }^{124,125}$ This evidence underscores the critical need for reliable, standardized measurement techniques, both for blood pressure (i.e., office blood pressure assessed with an automated device such as the BpTru) and psychosocial functioning (i.e., validated questionnaires). 
The third literature gap is the lack of prospective longitudinal cohort and experimental research; in particular, research that manipulates the independent psychosocial variables of interest. Currently, the majority of the literature has been based on cross-sectional research where measures are obtained at one point in time and with no manipulation of independent anger predictor variables of interest. These study designs do not provide the strongest evidence of causality. Prospective longitudinal cohort and experimental studies that manipulate anger and obtain measures at more than one time point would enable a more rigorous evaluation of these relationships. This lack of more robust evidence remains as a barrier to determining the existence and validity of a relationship between anger and blood pressure.

To summarize, literature gaps to the field of anger and blood pressure research include:

- A lack of consideration for potential racial/ethnic and sex/gender effects

- A lack of consistent use of validated and reliable self-report anger questionnaires

- A lack of consistent use of reliable blood pressure measurement techniques

- A lack of research based on prospective longitudinal cohort or experimental design; in particular, research that manipulates independent psychosocial variables of interest

These limitations must be addressed in future research in order to determine the existence and validity of a relationship between anger and blood pressure.

\section{Perceived Stress \& Blood Pressure}

\subsection{Historical Context \& Definitions}

The internalization of psychological stress has been argued as the most relevant for studying the psychobiological effects of stress on the body. ${ }^{126-129}$ More specifically, that "how an individual appraises stress, rather than the objective occurrence of stressful events, is what determines one's response to stressors". ${ }^{126,127,129}$ While external measures of stress are objective and easier to measure (i.e., a stressful event either occurred or did not occur) they cannot address the issue that stressful events elicit varying responses from individuals. For example, personality factors such as a strong commitment to self, an attitude of vigorousness toward the environment, a sense of meaningfulness and an internal locus of control (collectively referred to as "hardiness") have been identified as factors that predict illness onset due to stressful life events. ${ }^{130}$ This evidence 
outlines the existence of innate factors that trigger different internal stress responses and, moreover, that objective measures of stress could not have predicted the outcomes. For these reasons, perceived stress is an appropriate type of stress for investigating the contribution of stress to disease processes.

Richard Lazarus was the first to address appraised stress in 1966 using a conceptual psychological framework called the 'transactional model of stress.' ${ }^{126}$ Over time, through combined and sometimes competing efforts, Richard Lazarus and Sheldon Cohen contributed most significantly to our current understanding of appraised stress. ${ }^{127-129,131}$ Both teams developed tools to measure appraised stress including the Ways of Coping Checklist published in 1980 by the Lazarus team, ${ }^{132}$ the Hassles and Uplifts Scales published in 1981 by the Lazarus team ${ }^{133}$ and the Perceived Stress Scale (PSS) published in 1983 by the Cohen team. ${ }^{129}$ The Ways of Coping Checklist was a 68-item questionnaire, the Hassles Scale was a 117-item 'hassles' questionnaire, the Uplifts Scale was a 135-item 'uplifts' questionnaire and the PSS was a 4, 10 or 14-item perceived stress questionnaire (depending on the version). Together, the development of these questionnaires enabled researchers to begin measuring and understanding the contribution of appraised stress to different disease processes.

While all questionnaires measured some type of appraised stress and contributed to our current understanding of the concept, the PSS was unique in its brevity. The PSS was a 4, 10 or 14-item self-report questionnaire designed to measure the "degree to which situations in one's life appear[s] as stressful". ${ }^{129}$ More specifically, it was designed to measure how unpredictable, uncontrollable, and overloaded individuals appraised their lives to be. The PSS was sensitive to a variety of stressors, including "chronic stress deriving from ongoing life circumstances...stress from expectations concerning future events...stress from events not listed on a particular lifeevents scale, and to reactions to the specific events included on any scale". ${ }^{129}$ The questionnaire was brief, asked general questions and only required a junior high school education to be completed.

As the PSS reliably measured appraised stress using a brief, easily administrable questionnaire the PSS gave researchers the opportunity to more easily study the contribution of "nonspecific appraised stress [to] the etiology of disease and behavioural disorders" ${ }^{129}$ including hypertension and cardiovascular disease. Since its publication, the PSS has been used successfully in this 
regard, both for studying hypertension ${ }^{83,84,134,135}$ and cardiovascular disease. ${ }^{86,88}$ As a result of this work, perceived stress has been identified as an independent predictor of symptomatic coronary heart disease ${ }^{87}$ and has demonstrated association with blood pressure when studied alone ${ }^{83,84,134,135}$ and in conjunction with other measures of race-based discrimination. ${ }^{89}$ Thus, in addition to reliably measuring the "degree to which situations in one's life appear[ed] as stressful", ${ }^{129}$ the PSS enabled researchers to begin accurately studying the psychobiological effects of perceived stress on blood pressure.

Despite the brevity of the PSS questionnaire, the relationship between perceived stress and blood pressure has also been evaluated using non-validated perceived stress questionnaires. The decision to use non-validated perceived stress measurement tools over the validated PSS is rarely explained by authors. Non-validated perceived stress questionnaires are typically designed by researchers themselves, often consisting of a single question accompanied by a visual analog scale. Researchers may be inclined to use non-validated questionnaires due to easier administration and scoring. Support for these single-item stress questionnaires exists as several studies have cited positive associations between perceived stress and blood pressure ${ }^{78-82,85}$ The most famous stress study that has used this approach is the 2004 INTERHEART study. ${ }^{19}$ However, the contribution of these non-validated questionnaires has been both a benefit and a hindrance to the literature. The significant uptake and use of non-validated perceived stress questionnaires has increased research productivity in the field. However, the lack of theoretical background behind these non-validated questionnaires creates difficulty when attempting to assess what psychological construct these questionnaires are measuring. In summary, while the non-validated perceived stress literature supports an association between perceived stress and blood pressure, deriving clinical and scientific meaning from this literature is questionable.

Thus, to summarize, perceived stress addresses how an individual appraises the stress in his or her own environment. This type of psychological stress measurement is more appropriate than objective measures of stress as it considers the impact of the environment on an individual from the perspective of that individual. Research on appraised stress originated in 1966 with Richard Lazarus's 'transactional model of stress'. ${ }^{126}$ Together, Lazarus and Cohen developed the concept of appraised stress as well as several questionnaires to measure the construct. Among these was Cohen's Perceived Stress Scale. The combination of foundational research laid down by Lazarus and Cohen and the brevity/ease of administration of Cohen's Perceived Stress Scale equipped 
researchers with the necessary tools to effectively study the relationship between appraised stress and blood pressure. Both the PSS as well as non-validated perceived stress questionnaires have been used to evaluate the psychobiological effects of perceived stress on blood pressure. With half of the validated perceived stress literature and a majority of the non-validated perceived stress literature reporting a positive association with blood pressure, both questionnaire types have contributed to our understanding of the psychobiological effects of stress on the body and blood pressure.

\subsection{Evidence to Date on Perceived Stress \& Blood Pressure}

Overall, the available evidence has reported a positive association between perceived stress and blood pressure. Limitations to the perceived stress literature are similar to those reported in the anger literature. This includes mixed results, varying outcome measures, lack of evidence based on prospective longitudinal cohort or experimental studies that manipulate perceived stress and lack of pathophysiological mechanistic explanations. However, of most significant concern to the perceived stress field is the overwhelming use of non-validated psychometric questionnaires. In order to address this issue, the following literature review has been divided into studies that have used the validated perceived stress measures and those that have not. A summary of all perceived stress findings can be found in Table 6.

\subsubsection{Blood Pressure \& Validated Measures of Perceived Stress}

Since the development of Cohen's Perceived Stress Scale, 4 studies have examined the relationship between perceived stress and blood pressure. This has consisted of 3 cross-sectional studies $^{83,134,135}$ and 1 longitudinal cohort. ${ }^{84}$

\section{Validated Perceived Stress Measures: Positive Association}

Two of the 4 studies have reported a positive association between perceived stress as measured by the validated PSS and blood pressure. In 2011 Huang et al. evaluated PSS scores and nighttime blood pressure dipping pattern. ${ }^{83}$ Non-dipping is known as the absence of a normal nighttime decrease in blood pressure. Absence of this pattern has been associated with hypertensive target organ damage among both hypertensives and normotensives. ${ }^{136-139}$ Stratifying 307 Chinese essential hypertensives (52\% male) as either dippers or non-dippers, it was found that non-dippers were more likely to report a higher stress status. Similarly, decline in 
nighttime systolic or diastolic blood pressure was inversely related to PSS score. The second study reporting a positive association between PSS and blood pressure was the longitudinal Spokane Heart Study. ${ }^{84}$ One hundred and seventy male subjects asymptomatic for cardiovascular disease were evaluated at 2-year intervals for at least 6 years. The authors found that higher PSS scores were associated with higher levels of diastolic blood pressure at follow-up as measured by standardized manual blood pressure measurement. These 2 studies outline the current evidence in support of a positive association between perceived stress and blood pressure.

\section{Validated Perceived Stress Measures: No Association}

Two studies have found no association between PSS scores and blood pressure. In a recent crosssectional study of 102 Korean Americans (40\% male); PSS scores were not correlated with systolic or diastolic blood pressure when measured by an automated device. ${ }^{134}$ A second crosssectional study on 100 university students (38\% male), who participated in a mental arithmetic task, also reported no relationship between PSS scores and change in systolic or diastolic blood pressure. $^{135}$

\section{Validated Perceived Stress Measures: Summary}

Among the studies that used Cohen's validated PSS questionnaire, 2 studies reported a positive association and 2 studies reported no association. However, closer inspection of these studies suggests an overall positive association between perceived stress (as measured by the validated PSS) and blood pressure. This conclusion is based on larger sample sizes and use of a longitudinal cohort design in 1 of the 2 positive studies. A lack of generalizability from 1 of the

negative studies on American university students reinforces the above-mentioned conclusion. ${ }^{135}$ However, due to the low number of studies, much more research remains to be completed in order to determine the existence and validity of a relationship between perceived stress as measured by the PSS and blood pressure. Narrow racial/ethnic diversities also cannot rule out possible underlying racial/ethnic effects. Future efforts should focus on the inclusion of subjects from more diverse racial/ethnic backgrounds, as well as continued recruitment of male and female study participants. 


\subsubsection{Blood Pressure \& Non-Validated Measures of Perceived Stress}

Ten studies have investigated associations between perceived stress as measured by a nonvalidated perceived stress questionnaire and blood pressure. This includes 1 longitudinal ${ }^{85}$ and 9 cross-sectional studies. ${ }^{78-82,140-143}$

\section{Non-Validated Perceived Stress Measures: Positive Association}

Six studies have reported a positive association between perceived stress and blood pressure. This evidence consists of 1 longitudinal ${ }^{85}$ and 5 cross-sectional studies. ${ }^{78-82}$

Examining the 5 cross-sectional studies in more detail, it can be seen that 2 evaluated blood pressure as a continuous variable ${ }^{78,82}$ and 3 made perceived stress a categorical variable and studied blood pressure levels between 2 groups for their primary analyses. ${ }^{79-81}$ All studies assessed perceived stress using a 1 item questionnaire (i.e., $0=$ low stress to $10=$ high stress) and recruited large sample sizes across a wide range of subject demographics. All reported the use of reliable blood pressure measurement methods (4 studies used ambulatory and 1 used home blood pressure monitoring). One study assessed blood pressure and perceived stress simultaneously at 30-minute intervals using daytime ambulatory blood pressure monitoring. ${ }^{81}$ Based on this information, these 5 cross-sectional studies provide convincing evidence in support of a positive association between perceived stress (as measured by non-validated questionnaires) and blood pressure. At minimum, this evidence sets the stage for further investigation with the use of validated perceived stress questionnaires.

One longitudinal study also reported a positive association. Perceived stress and risk of hypertension development were studied using prospectively collected cohort data from the Copenhagen Heart Study. ${ }^{85}$ Following 7066 male and female subjects for approximately 10 years, the authors found that high perceived stress was associated with a greater risk of hypertension diagnosis at follow-up compared to low perceived stress. The authors measured perceived stress in terms of stress intensity and stress frequency using a 2-item questionnaire. Stress scores were then categorized into low, medium and high perceived stress. The large sample size and substantial follow-up time reinforce these results and suggest that non-validated 
perceived stress could play some role in increasing the long-term risk for hypertension development.

\section{Non-Validated Perceived Stress Measures: No Association}

Three cross-sectional studies have reported no association between perceived stress and blood

pressure. ${ }^{140,141,143}$ Two studies assessed perceived stress based on a single question ${ }^{140,141}$ and 1 study developed more elaborate questionnaires. ${ }^{143}$ All studies except 1 used a reliable method of blood pressure measurement. ${ }^{143}$ The sample populations under study are more heterogeneous compared to the positive association studies. Nonetheless, these 3 studies outline that not all of the non-validated perceived stress literature supports a positive association between perceived stress and blood pressure.

\section{Non-Validated Perceived Stress Measures: Negative Association}

One cross-sectional study reported a negative association between perceived stress and blood pressure. ${ }^{142}$ The authors measured perceived stress among 1601 Caucasian males using a 1-item questionnaire. While drawing conclusions based on 1 study is difficult, this evidence does outline the existence of different types of relationships between perceived stress as measured by non-validated questionnaires and blood pressure.

\section{Non-Validated Perceived Stress Measures: Summary}

Overall, more studies using non-validated measures of perceived stress reported positive associations between perceived stress and blood pressure ( 6 out of 10 studies, including 1 longitudinal study). The methodological quality of positive studies was generally higher than the negative or no association studies. However, the lack of a validated perceived stress questionnaire makes generalizing and comparing results very challenging. At a minimum, these studies have set the stage for further investigation with validated perceived stress questionnaires. Given the narrow racial/ethnic diversity of studies in this section (due in part to lack of reporting), it is possible there are underlying racial/ethnic effects mediating these relationships. Future efforts should focus on the inclusion and reporting of subjects from more diverse racial/ethnic backgrounds and continue recruitment of male and female study participants. 


\subsubsection{Synthesizing the Evidence}

Based on evidence from both the validated and non-validated perceived stress literature, it appears that perceived stress is positively associated with blood pressure in some capacity. This

conclusion aligns with what is observed in the cardiovascular literature. ${ }^{86-90}$ However, this conclusion has primarily been based on cross-sectional research where the independent variable (perceived stress) was not manipulated and variables were measured only once. Thus, these results must be interpreted accordingly. Prospective longitudinal cohort and experimental studies that employ more robust methodology and manipulate the independent psychosocial variables of interest are necessary to clarify this literature. The existence of underlying racial/ethnic effects is still unclear. Therefore, future efforts should also focus on the inclusion of subjects from diverse racial/ethnic backgrounds, as well as continued recruitment of male and female study participants.

\section{Literature Gaps}

Based on the evidence presented above, the major literature gaps facing the perceived stress and blood pressure field are:

- A lack of research based on validated perceived stress questionnaires

- A lack of research based on prospective longitudinal cohort or experimental design; in particular, research that manipulates independent psychosocial variables of interest

- A lack of reporting/enrolment of subjects from diverse racial/ethnic backgrounds

These limitations must be addressed in future research in order to confirm the existence and validity of a relationship between perceived stress and blood pressure

\section{Moving Forward: Prospective Longitudinal Cohort Designs}

The preceding sections have reviewed the literature on the relationships between anger, perceived stress and blood pressure. Almost all of this literature has been based on crosssectional data that has not manipulated independent psychosocial variables of interest or measured variables at more than one point in time. The major obstacle faced by the current literature is the inability to establish whether change in psychosocial functioning is indeed 
associated with change in blood pressure. More specifically, the lack of strong study designs and manipulation of psychosocial predictor variables make it difficult to provide strong evidence of causality. Thus, in addition to the overwhelming division reported in the literature, the restriction to cross-sectional observational studies further prevents the formulation of robust conclusions.

However, study designs such as prospective longitudinal cohort designs that manipulate independent psychosocial variables of interest, could be used to test these relationships more directly. More specifically, by manipulating anger or perceived stress it would be possible to evaluate whether changes in psychosocial variables are associated with changes in blood pressure. Therapeutic psychological interventions are one tool by which anger or stress could be manipulated in a prospective longitudinal cohort setting. Preliminary evidence from the literature has outlined the potential use of therapeutic psychological interventions for this purpose.

\subsection{Therapeutic Interventions}

\section{Therapeutic Interventions: Anger}

Preliminary evidence exists in support of using therapeutic psychological interventions to test the relationship between anger and blood pressure. Five studies that used anger management, relaxation or stress reduction therapies demonstrated that improvements in anger were associated with improvements in blood pressure. ${ }^{144-148}$

Among the anger management research, it was found that male and female subjects with mild essential hypertension who completed a 6-week anger management program demonstrated significantly lower casual diastolic blood pressure compared to no-treatment controls. ${ }^{147}$ Two other studies demonstrated that concurrent decreases in verbal and physical aggressive behaviours and increases in constructive anger verbal behaviour successfully reduced resting ambulatory blood pressure. ${ }^{144,146}$ Of note, one of these 2 studies was also directly interested in clarifying the relationship between psychosocial risk factors and blood pressure. ${ }^{144}$ Finally, through the use of stress and type-A management interventions, unmedicated mildly hypertensive male subjects demonstrated reductions in systolic blood pressure reactivity that were associated with reductions in measures of anger expression inward and expression outward. ${ }^{148}$ Together, this evidence outlines the utility of therapeutic psychological interventions such as anger management to study the relationship between anger and blood pressure. 
Relaxation therapy has also been used effectively to manipulate anger to evaluate its association with blood pressure. ${ }^{145}$ One study enrolled male subjects and allocated them to either relaxation or no-treatment control. Reductions in anger-related hostility were significantly associated with reductions in standing systolic blood pressure. Interestingly, these results were most significant for subjects high in norepinephrine, suggesting a possible pathophysiological link between relaxation, anger and blood pressure. This was also one of the few studies directly interested in clarifying the relationship between psychosocial risk factors and blood pressure. This relaxation intervention study provides further support for the use of therapeutic psychological interventions in a prospective longitudinal cohort setting to study the relationship between anger and blood pressure.

\section{Therapeutic Interventions: Perceived Stress}

One study has provided evidence supporting the use of therapeutic psychological interventions to study the relationship between perceived stress and blood pressure. In 2003, McCraty et al. evaluated the effect of a workplace stress-reduction program on emotional health and blood pressure among 38 hypertensive employees. ${ }^{149}$ The authors reported that decreases in stress symptoms were significantly correlated with reductions in systolic blood pressure as measured by an automated device. Although limited in evidence, this preliminary study provides support for using a psychological therapeutic intervention to test the relationship between perceived stress and blood pressure.

\section{Therapeutic Interventions: Summary}

Preliminary evidence has outlined the appropriateness of using a therapeutic psychological intervention as a tool to manipulate stress and anger in order to more directly test their association with blood pressure. Limitations to the current evidence include its preliminary nature, lack of consistent use of validated self-report questionnaires, lack of reliable blood pressure measurement techniques and lack of standardized therapies. Nonetheless, this information provides the necessary framework for further testing with more rigorous methodology. 


\subsection{Mindfulness-Based Stress Reduction}

Mindfulness-Based Stress Reduction (MBSR) is a standardized group stress reduction therapy that could be used as a tool to test the association between anger, stress and blood pressure.

In 1985, Jon Kabat-Zinn introduced MBSR to treat chronic pain. ${ }^{150}$ Today, MBSR is used to also treat anxiety, ${ }^{151-155}$ depression, ${ }^{151,154,156-159}$ fibromyalgia, ${ }^{159-161}$ binge-eating ${ }^{162}$ and psoriasis. ${ }^{163}$ Currently, MBSR can be found across Canada at major hospitals and local community health centers for the treatment of the abovementioned conditions. MBSR teaches individuals mindfulness "as an approach to adapt to stress, pain and illness". ${ }^{1}$ Improved mindfulness enables individuals to "more clearly see the full context of a situation, have access to a broader range of emotional responses and therefore cope with stressful situations more effectively"., 153 In addition to the above-mentioned conditions, MBSR has also demonstrated efficacy in improving components of anger ${ }^{164-166}$ and perceived stress ${ }^{167-171}$ when measured using validated questionnaires. The psychological construct known as "mindfulness" has been proposed as a mediating mechanism for improving both perceived stress ${ }^{170,172}$ and anger. ${ }^{173-175}$

MBSR can be summarized as an established, standardized therapy with evidence documenting its efficacy for improving anger and perceived stress. Combining this information with previously discussed evidence citing the success of other therapeutic psychological interventions illustrates that MBSR has potential to be used in a prospective longitudinal cohort setting to more directly test associations between anger, perceived stress and blood pressure.

\subsection{Summary}

To summarize, preliminary evidence exists in support of using therapeutic psychological interventions to test the relationship between anger, perceived stress and blood pressure. Mindfulness-based stress reduction is a potential tool that could be used to manipulate anger and perceived stress in a prospective longitudinal cohort setting. Using MBSR might clarify the existence and validity of a relationship between anger, perceived stress and blood pressure. 


\section{Rationale \& Hypothesis}

\subsection{Rationale}

Evidence exists suggesting an association between anger, perceived stress and blood pressure. However, much of this evidence is divided and has been based on cross-sectional research measured at one point in time and with no manipulation of independent psychosocial variables of interest. Prospective longitudinal cohort research that includes an intervention designed to impact anger and perceived stress would add more robust evidence to the literature and may help to clarify currently divided findings.

Therapeutic psychological interventions are one tool by which the relationship between anger, perceived stress and blood pressure can be tested more directly in a prospective longitudinal cohort setting. Preliminary evidence has outlined the appropriateness of this approach. MBSR is one intervention that could be used in a prospective longitudinal cohort study of this kind. MBSR was previously tested prospectively for its efficacy in blood pressure lowering therefore providing an opportunity to test the relationship between anger, perceived stress and blood pressure.

This study used MBSR as a tool to manipulate anger and perceived stress to evaluate their association with blood pressure. More specifically, the objective of this study was to determine the existence of an association between change in anger (measured by the State-Trait Anger Expression Inventory) and change in perceived stress (measured by Cohen's Perceived Stress Scale) with change in ambulatory blood pressure among unmedicated stage- 1 hypertensive individuals who underwent a standard 8-week MBSR program.

Findings from this prospective longitudinal cohort study will contribute to the current understandings of anger and perceived stress as suspected risk factors for hypertension development.

\subsection{Hypothesis \& Objectives}

The objective of this study was to establish the existence of an association between change in anger and perceived stress with change in ambulatory blood pressure among untreated stage-1 hypertensive subjects who underwent a standard 8-week MBSR protocol. 
It was expected that associations between psychosocial stressors and blood pressure would occur while subjects were awake, undergoing daily activities and managing psychosocial stressors. Therefore, daytime and 24-hour ambulatory blood pressures were selected as primary outcome measures in this study. The primary hypothesis tested was that change in psychosocial variables thought to be linked to blood pressure (trait anger, trait anger temperament, trait anger reaction, anger expressed in, anger expressed out and perceived stress) would demonstrate an association with change in daytime and 24-hour ambulatory blood pressure among untreated stage-1 hypertensive subjects who underwent a standard 8-week MBSR protocol.

Based on the possibility that blood pressure could be affected by anger or stress while subjects slept and subconsciously replayed the events of their day through their mind, the secondary hypothesis tested whether change in the above-mentioned psychosocial variables would demonstrate an association with change in nighttime blood pressure among untreated stage-1 hypertensive subjects who underwent a standard 8-week MBSR protocol.

Three anger constructs (anger control in, anger control out and the anger expression index) received less support from the literature. Thus, they were investigated for exploratory purposes. The specific exploratory hypotheses tested for the existence of an association between change in exploratory anger measures (anger control in, anger control out and the anger expression index) and change in daytime, 24-hour and nighttime blood pressure among untreated stage-1 hypertensive subjects who underwent a standard 8-week MBSR protocol.

Additional exploratory hypothesis tested for the existence of sex differences by re-testing all of the abovementioned hypotheses after stratifying by sex.

State anger was not investigated in this study due to the specific situational nature of the construct and the lack of support from the literature.

Test of the hypotheses are outlined in the Methods section in the next chapter. 


\section{Chapter 2 Methods}

\section{Study Design}

Subjects included in this thesis study were from the HARMONY trial (ㅍypertension $\underline{\text { Analysis of }}$

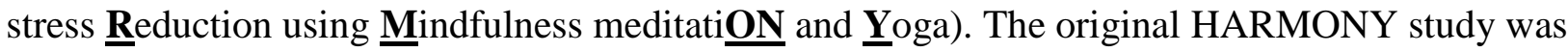
a prospective wait-list randomized controlled trial designed to test the use of mindfulness based stress reduction (MBSR) for blood pressure lowering among stage-1 unmedicated hypertensive subjects. The specific objective of HARMONY was to examine the effect of a standard 8-week MBSR therapy program on ambulatory blood pressure among stage- 1 unmedicated hypertensives, as has been described. ${ }^{1}$

Using a prospective longitudinal cohort within-group design with two time points (baseline and after MBSR), this thesis study used data from the HARMONY study to test the above-mentioned hypotheses. The specific objective of this study was to examine the existence of an association between change in anger and perceived stress with change ambulatory blood pressure among untreated stage-1 hypertensive subjects who underwent a standard 8-week MBSR protocol.

Based on the understanding that behavioural interventions often result in small to moderate effect sizes, and that all participants in the HARMONY study underwent all measurements and participated in the mindfulness intervention, the within-group design was selected to increase the sample size and thus the possibility of detecting an association. Thus while HARMONY was conducted as a wait-list randomized controlled trial, this thesis evaluated all subjects before and after MBSR.

\section{Participants}

Participants for the HAROMY study were recruited from referring physicians, advertisements in local newspapers and posters at local hospitals. Eligible subjects were between the ages of $20-$ 75 years of age. Main inclusion criteria included a diagnosis of stage- 1 hypertension as confirmed by ambulatory blood pressure monitoring. Specific ambulatory blood pressure eligibility criteria included daytime ambulatory blood pressure $\geq 135 / 85 \mathrm{mmHg}$ or 24-hour 
ambulatory blood pressure $\geq 130 / 80 \mathrm{mmHg}$. Subjects could qualify based on either their systolic or diastolic blood pressure.

In addition to a stage-1 diagnosis, subjects were required to be untreated (no antihypertensive medication) for at least 6 months prior to the baseline screening visit. Additional exclusion criteria included a diagnosis of diabetes, secondary hypertension or target organ damage. Labs and echocardiograms were collected to rule out target organ damage and diabetes. Study requirements also included a willingness and ability to participate in the MBSR program and attend all necessary study visits and safety blood pressure evaluations. More detailed study inclusion and exclusion criteria can be found in Table 7.

\section{Screening \& Blood Pressure Measurement}

All study visits took place at Sunnybrook Health Sciences Centre, Toronto, Ontario. All MBSR classes were conducted at Toronto General Hospital, Toronto, Ontario. Study subjects were not given incentive for participation in the study; however, they were reimbursed for parking expenses. All experimental protocols and informed consent processes were approved by Research Ethics Boards at both institutions and conducted according to the principles of Good Clinical Practice and the Declaration of Helsinki.

Office blood pressure measurements were performed by trained research staff using an automated blood pressure measurement device (BpTru Limited., Coquitlam, BC, Canada). Six blood pressure readings were taken at 2-minute intervals; the first reading was discarded with the average of the last 5 representing the average seated office automated blood pressure. Office automated blood pressure was used to screen for stage-1 hypertension. All other office blood pressure checks were for blood pressure safety monitoring purposes.

Ambulatory blood pressure monitoring determined study eligibility via either baseline daytime or 24-hour blood pressure results (Model 90207, Spacelabs Medical Inc., Redmond, WA). Subjects wore an ambulatory blood pressure monitor for 24 hours. Blood pressure readings were taken at 15-minute intervals during the day (07:00 - 11:00) and at 30-minute intervals at night (11:00 - 07:00). Daytime and nighttime ambulatory blood pressure readings were adjusted according to participant diaries. Research staff were trained on all proper blood pressure 
measurement protocols and regularly supervised. Only cuff sizes appropriate for subjects' arm circumferences were used.

\section{Study Flow}

At study entry all participants underwent anthropomorphic measurements and were administered Cohen's Perceived Stress Scale (PSS) and the State-Trait Anger Expression Inventory-2 (STAXI-2). All subjects were scheduled to begin MBSR within 4-12 weeks of the baseline visit. Participants received no mindfulness training during the wait-list period. All subjects returned after MBSR and underwent ambulatory blood pressure monitoring, office blood pressure measurements, anthropomorphic measurements and completed the PSS and STAXI-2. Following this, participants began a follow-up period and returned 12 weeks after MBSR for a final close out ambulatory blood pressure monitoring.

\section{Intervention}

The MBSR program implemented in the HARMONY study has been described elsewhere but is explained below. ${ }^{1}$ MBSR is a "multi-component group therapy that provides systematic training in mindfulness meditation as a self-regulation approach to stress reduction and emotion management. [It] provides training in formal meditation approaches with the primary goal of cultivating psychological resilience and resistance to stress. This is achieved through fostering the quality of "mindfulness", defined as the capacity to intentionally be in the present moment without judgment". 1, 176 The therapy "teaches attendees to approach stressful situations "mindfully"; allowing them to identify what is occurring in their bodies and minds and to step back from thoughts and feelings during stressful situations. This affords participants the opportunity to make wise choices with respect to managing stressful situations, such as choosing to avoid engaging in anxious worry that may otherwise escalate into a cycle of stress reactivity and contribute to more emotional distress".,1,150,176-178

In the study, MBSR was delivered over a 9 week period, consisting of one introductory session and 8 weekly 2.5 hour sessions. All MBSR therapy was administered at Toronto General Hospital. There was also a 6 hour silent retreat held on a Saturday or Sunday between the $6^{\text {th }}$ and $7^{\text {th }}$ week of the program. The MBSR program "incorporate[d] four major therapeutic elements: formal meditation, informal mindfulness practice (such as bringing mindfulness to daily 
activities), psycho-educational activities, and self-monitoring/reflection exercises". ${ }^{1}$ Each week participants learned new techniques to assist with cultivating the state of mindfulness.

Techniques included mindfulness meditation, mindful walking, mindful eating, loving-kindness meditation, yoga etc. In addition to physical activities, each class included group discussion. Progress through the MBSR program was captured via weekly homework logs which were faxed weekly from Toronto General Hospital to Sunnybrook Health Sciences Centre.

\section{Outcomes}

\subsection{Primary}

The primary outcome measure of this thesis study was post intervention 24-hour and daytime systolic and diastolic blood pressure as measured by 24-hour ambulatory blood pressure monitoring. Additional measures included post-intervention perceived stress and anger as measured by the PSS and STAXI-2. The primary objective of this study was to establish whether changes in primary predictor variables (post intervention trait anger, trait anger temperament, trait anger reaction, anger expression inwards, anger expression outwards and perceived stress) were related to changes in daytime or 24-hour blood pressure after participants completed an 8week MBSR program.

\subsection{Secondary}

The secondary outcome measure was post-intervention nighttime blood pressure as measured by 24-hour ambulatory blood pressure monitoring. Secondary objectives included establishing whether changes in primary predictor variables (post intervention trait anger, trait anger temperament, trait anger reaction, anger expression inwards, anger expression outwards and perceived stress) were related to changes in nighttime blood pressure after participants completed an 8-week MBSR program.

\subsection{Exploratory}

Post-intervention daytime, 24-hour and nighttime blood pressure were also used as outcome measures for the exploratory analyses. The exploratory objectives included establishing whether change in exploratory predictor variables (post intervention anger control inwards, anger control 
outwards and the anger expression index) were related to change daytime, 24-hour and nighttime blood pressure.

Additional exploratory objectives included establishing the existence of sex any differences.

\section{Measures}

\subsection{State Trait Anger Expression Inventory (STAXI)}

The STAXI is a validated self-report questionnaire that measures anger as outlined by Spielberger's theory: anger experience (state and trait anger) and anger expression (expression and control of anger). The STAXI is designed to provide "easily administered and objectively scored measures of the experience, expression and control of anger for adolescents and adults". ${ }^{2}$ Completion of the STAXI requires $12-15$ minutes and a $6^{\text {th }}$ grade reading level. The second version of the STAXI (the STAXI-2) was used in this study.

The STAXI-2 consists of 57 questions that use a 4-point Likert response format. The Likert response format ranges from Not at all (1) to Very much so (4) for the State Anger questions, and Almost never (1) to Almost always (4) for the Trait Anger, Anger Expression and Anger Control questions. Higher ratings reflect a greater intensity of angry feelings or higher frequencies to experience, express, suppress or control anger. In total, the questionnaire consists of 6 scales and 5 subscales (subscales are included in square brackets): State anger (S-Ang) [feeling angry (SAng/F), feel like expressing anger verbally (S-Ang/V), feel like expressing anger physically(SAng/P)], trait anger (T-Ang) [trait anger temperament (T-Ang/T), trait anger reaction(T-Ang/R)], anger expression in (AXI), anger expression out (AXO), anger control in (ACI), anger control out (ACO) and the anger expression index (AXX). A summary can be found in Table 8 .

The 15-item State Anger scale measures the intensity of angry feelings and the extent to which a person feels like expressing anger at a particular time. As the state-anger subscale was not investigated in this study it will not be described in further detail. The Trait Anger scale includes two subscales, Angry Temperament and Angry Reaction. Trait Anger scale consists of 10 questions that measure how often angry feelings are experienced over time. The Angry Temperament subscale consists of 4 questions that measure the disposition to experience angry feelings without provocation. The four-item Angry Reaction subscale measures the frequency 
that angry feelings are experienced in situations that involve frustration and/or negative evaluations. The STAXI also includes 8-question sections on anger expression in which measures how often angry feelings are experienced but not expressed, anger expression out which measures how often angry feelings are expressed using verbally or physically aggressive behaviour, anger control in which measures how often a person attempts to control angry feelings by calming down or cooling off, and anger control out which measures how often a person controls the outwards expression of angry feelings. Finally, the Anger Expression Index combines the anger expressed in, anger expressed out, anger control in and anger control out scales to yield an omnibus measure of how one reacts or behaves when angry.

The STAXI-2 and its subscales have been extensively studied on normative and psychiatric samples and have demonstrated good internal consistency and convergent validity. Test-retest reliability of the STAXI-2 scales have also received support. ${ }^{179,}{ }^{180}$ As reported by Spielberger, ${ }^{104}$ alpha coefficients (measures of internal consistency) for state and trait anger were high across all scales and subscales ( 0.84 or higher) except for trait anger reaction which was 0.76 and 0.73 for normal females and males, respectively. In terms of convergent validity, Trait Anger was found to be highly correlated with the Buss-Durkee Hostility inventory total score ${ }^{181}$ and the Hostility $^{182}$ and Overt Hostility ${ }^{183}$ scales of the Minnesota Multiphasic Personality Inventory. ${ }^{184}$ Both state anger and trait anger correlated with neuroticism and psychoticism scales from the Eysenck Personality Questionnaire ${ }^{185}$ and the state and trait anxiety and curiosity subscales of the State Trait Personality Inventory. ${ }^{186}$ Alpha coefficients for anger expression in, anger expression out, anger control in, anger control out and the anger expression index have also been reported to be adequate and uniform across all scales and subscales ( 0.73 or higher $).{ }^{2}$ In terms of convergent validity, the anger expression scales have demonstrated correlations with other anger measures (such the Harburg Anger-In/Anger-Out Scale) ${ }^{94}$ as well as other personality measures (such as the state and trait anxiety and curiosity subscales of the State Trait Personality Inventory). ${ }^{104}$ In terms of construct validity, Spielberger also demonstrated an association between anger expression scores and written responses to hypothetical anger scenarios. ${ }^{2}$

\subsection{Cohen's Perceived Stress Scale}

Perceived stress was measured using the Perceived Stress Scale (PSS) developed by Cohen, Kamarck, and Mermelstein. ${ }^{129}$ The PSS is the only empirically established index of general 
stress appraisal. In this study, the 14-item version was administered to assess the degree to which participants appraised their current situations as unpredictable, uncontrollable or overwhelming. The PSS includes easy-to-understand questions about participants' stressful thoughts or feelings related to situations in their lives within the last month. Each item is rated on a 5-point Likert scale ranging from Never (0) to Very Often (4). A total PSS score is calculated by reverse-scoring the seven positive items and then summing scores for all 14 items. Questions $4,5,6,7,9,10$, and 13 are the positively stated items. PSS scores can range from 0 to 56 . A junior high school education is required to take the PSS. As the PSS is not a diagnostic instrument there are no cut-offs; ${ }^{131}$ there can only be comparisons with other samples. ${ }^{129,187}$

The PSS has effectively assessed perceived stress among healthy ${ }^{129,188}$ and psychiatric populations ${ }^{189}$ and has been used in collaboration with external measures of stress such as cortisol. ${ }^{190,191}$ With internal consistency scores (alpha coefficients) ranging from 0.84 to 0.86 , the PSS has demonstrated high levels of reliability and validity. ${ }^{129,}{ }^{187}$ This finding has been supported by other studies. ${ }^{191-194}$ The PSS has demonstrated high convergent validity by correlating with specific life-event impact scores. ${ }^{129}$ High construct validity has also been demonstrated with studies on smoking-cessation. More specifically, PSS scores have been positively associated with health, health service utilization, health behaviours, life satisfaction and help seeking. ${ }^{187}$

\subsection{Ambulatory Blood Pressure Monitoring}

Twenty-four hour ambulatory blood pressure monitoring (ABPM) was used for the primary, secondary and exploratory outcome measures in this study. ABPM is referred to as the gold standard in blood pressure measurement for several reasons. First, is the reduction in measurement error due to the entirely automated measurement process. Second, is the elimination of observer bias as blood pressure is measured while subjects undergo their regular daily activities with no health care providers present. This type of blood pressure measurement reduces blood pressure variability and associated observer effects such as white coat and masked hypertension. Finally, by taking approximately 80 blood pressure measurements over 24 hours, ABPM reduces variability and distills a blood pressure average closer to an individual's 'true' blood pressure. Compared to other blood pressure measurement methods, ABPM results correlate much better with long term target organ damage and are better a predicting 
cardiovascular risk. ${ }^{195}$ ABPM results have also demonstrated association with various types of psychosocial functioning. ${ }^{109,} 196$

\section{Statistical Methods}

\subsection{Baseline Correlations}

Comparisons of baseline data between male and female study subjects were evaluated using unpaired t-tests or chi-squared tests. Baseline correlations between psychosocial variables and daytime, 24-hour and nighttime blood pressure were evaluated using Pearson product moment zero order correlation matrices. Spearman ranked correlation matrices were used to evaluate any correlations with trait anger temperament due to right skewed data. The same analysis approach was used for all primary, secondary and exploratory analyses. All analyses were repeated after stratifying by sex.

\subsection{Pre-Post Changes}

Paired t-tests were used to examine changes from pre to post intervention for blood pressure and the psychometric variables of interest. 'Pre' and 'post' referred to baseline measures and measures taken after the MBSR program, respectively. All analyses were repeated after stratifying by sex. A sign test was used to evaluate pre-post differences of trait anger temperament due to its non-normal baseline distribution.

Anger scores were also dichotomized according to those who scored $<75^{\text {th }}$ percentile and those who scored $\geq$ the $75^{\text {th }}$ percentile. According to work completed by Spielberger, "individuals with anger scores above the $75^{\text {th }}$ percentile experience and/or express angry feelings to a degree that may interfere with optimal functioning ${ }^{197}$... The anger of these individuals may contribute to difficulties in interpersonal relationships or dispose them to develop psychological or physical disorders". ${ }^{2}$ Furthermore, "high anger expression inwards scores, especially when associated with low anger expression outwards and high levels of anxiety, have been shown to be associated with elevated blood pressure and hypertension ${ }^{57,66,198} \ldots$ Very high anger scores (i.e., $\geq 90^{\text {th }}$ percentile) on both the [anger expressed out] and [anger expressed in] scores have been associated with vulnerability to coronary artery disease and heart attacks". ${ }^{2}$ Based on this information, anger scores were dichotomized to examine changes from pre to post intervention according to the $75^{\text {th }}$ percentile threshold. McNemar's test for paired proportions was used to test 
whether the proportion of those scoring $<$ or $\geq 75^{\text {th }}$ percentile on the anger measures changed from pre to post intervention. Cut-offs for the $75^{\text {th }}$ percentile or greater were taken from the STAXI-2 professional manual and applied to each category of analysis respectively (i.e., males and females, females only and males only). Exact values from the test have been reported. All analyses were repeated stratifying by sex.

\subsection{Psychometric Properties}

Cronbach's alpha was calculated to assess internal consistency. ${ }^{199}$ Standardized values have been reported.

\subsection{Change Correlations}

Change correlations were evaluated by incorporating pre to post change scores into Pearson product moment zero order correlation matrices. This consisted of all psychosocial variables with daytime, 24-hour and nighttime blood pressure. Spearman ranked correlation matrices were used to evaluate any change correlations with trait anger temperament due to right skewed change score data. The same analysis approach was used for all primary and secondary analyses. All analyses were repeated after stratifying by sex.

\subsection{Multivariate Linear Regressions}

Primary multivariate linear regression analyses were used to evaluate whether psychosocial functioning (post intervention trait anger, trait anger temperament, trait anger reaction, anger expression inwards, anger expression outwards and perceived stress) was associated with ambulatory blood pressure (24-hour and daytime blood pressure), after adjusting for baseline blood pressure and baseline psychosocial values and other covariates.

Based on previous work from our lab the following variables were selected as covariates and included in all models ${ }^{200-202}$ : age, sex (female), baseline body mass index (BMI), race (Caucasian=yes), family history of premature cardiovascular disease (yes), baseline drinking of $\geq$ 10 drinks/wk. (yes), education level > post-secondary (yes), regular practice of relaxation at baseline (yes) and regular exercise at baseline (yes). Dependent variables in the primary analysis included daytime and 24-hour systolic and diastolic blood pressure. Dependent variables in the secondary analysis included nighttime systolic and diastolic blood pressure. Dependent variables 
in the exploratory analysis included daytime, nighttime and 24-hour systolic and diastolic blood pressure. Baseline anger, perceived stress and blood pressure were included as covariates in all models to account for the Law of Initial Values. ${ }^{203}$

It should be noted that post intervention trait anger temperament scores were slightly skewed and not amenable to transformation. Therefore, a modified full main effects model was evaluated for exploratory purposes. In this modified model, post intervention trait anger temperament scores were stratified into quartiles and used as predictor variables in the model. This was done for exploratory purposes to compare findings with the main model.

Secondary multivariate linear regression analyses evaluated whether changes in primary psychosocial variables were associated with changes in nighttime blood pressure. Additional anger measures (specifically anger control inwards, anger control outwards and the anger expression index) were evaluated in the exploratory multivariate linear regression analyses to establish the existence of any associations with daytime, 24-hour or nighttime blood pressure. All analyses (primary, secondary and exploratory) were repeated after stratifying by sex. The same covariates were used in all analyses.

\subsection{Effect Sizes}

Standardized regression coefficients were generated to evaluate standardized effects of the primary predictor variables (trait anger, trait anger temperament, trait anger reaction, anger expression inwards, anger expression outwards and perceived stress) on the primary outcome variables (24-hour and daytime systolic and diastolic blood pressure). Standardized regression coefficients were also generated for any anger or stress predictor variables that emerged as statistically significant in the secondary or exploratory analyses.

\subsection{Post Hoc-Analyses}

Post-hoc analyses after study completion included evaluating changes in anthropomorphic measurements, drinking habits and exercise levels from pre to post intervention. This was accomplished using paired t-tests and McNemar's test for paired proportions.

\subsection{Software}

Statistical analyses were performed using SAS version 9.3 (SAS Institute Inc., Cary, NC). 


\section{Chapter 3 Results}

\section{Study Details}

\subsection{Participant Flow}

In total 101 subjects were included in the study. Four hundred and eighty-three individuals were screened for study eligibility and 254 were excluded by telephone. The most common reason for telephone exclusion was current or recent antihypertensive therapy. From the remainder, 51 declined to participate and 77 were not stage-1 hypertensive upon ambulatory blood pressure monitoring.

Of the 101 subjects enrolled in the study 99 completed a pre intervention ambulatory blood pressure monitoring, 96 completed a pre intervention STAXI questionnaire and 90 completed a pre intervention PSS. Eight-five subjects completed a post intervention ambulatory blood pressure monitoring, 76 subjects completed a post intervention STAXI questionnaire (Note: only 75 subjects completed the trait-anger reaction subscale due to missing information in one questionnaire) and 86 subjects completed a post intervention PSS questionnaire. All subjects who had complete pre-post datasets were included in this study.

\subsection{Recruitment}

Age-eligible participants were recruited from May 2008 to December 2010. After the baseline screening visit, participants returned for study visits every 12 weeks for 6 - 10 months.

\subsection{Antihypertensive Treatment}

Nine subjects were advised to start antihypertensive therapy during the study due to the development of stage-2 hypertension. Subjects prescribed therapy continued in the study. Blood pressure data from subjects initiated on antihypertensive therapy was censored from the point of antihypertensive initiation onward. 


\section{Baseline Data}

All subjects with available pre-post data were included in the analysis. All subjects were stage-1 hypertensive and untreated for at least 6 months prior to study entry. The mean 24-hour ambulatory blood pressure for all participants at baseline was $135 / 81 \mathrm{mmHg}$ (SD 7.5/5.6). Mean daytime ambulatory blood pressure was 141/86 $\mathrm{mmHg}$ (SD 7.5/6.0) and mean nighttime ambulatory blood pressure was $122 / 71 \mathrm{mmHg}$ (SD 10.31/7.0) at baseline. The sample was approximately two thirds female with $82 \%$ of subjects self-identifying as Caucasian. A summary of this information can be found in Table 9. Overall, the cohort was fairly healthy and highly educated. Baseline psychosocial data can be found in Table 10. Percentile ranking of STAXI-2 anger scores demonstrated that the cohort was average in terms of anger levels. Perceived stress levels were also comparable to PSS scores from the normative samples used to develop the PSS questionnaire. ${ }^{129}$

Details regarding MBSR class attendance and homework completion were also collected. Out of 8 MBSR classes (excluding an introductory session and silent retreat) subjects attended an average of 6 classes (SD 1.5) and completed 32 minutes (SD 15.2) of additional MBSR homework per day (Table 11).

\subsection{Baseline Correlations}

There were no significant correlations between baseline primary psychosocial variables (trait anger, trait anger temperament, trait anger reaction, anger expressed inwards, anger expressed outwards or perceived stress) and baseline daytime or 24-hour or systolic or diastolic blood pressure (See columns labelled 'All Subjects' in Table 12 and Table 13). There were also no significant correlations between baseline primary psychosocial variables and baseline nighttime blood pressure (See column labelled 'All Subjects' in Table 14).

Among the baseline exploratory psychosocial measures (anger control inwards, anger control outwards and the anger expression index) only baseline anger control inwards was positively correlated with baseline daytime systolic blood pressure $(\mathrm{p}=0.0429)$. A negative correlation between baseline anger expression index and baseline daytime systolic blood pressure approached statistical significance $(\mathrm{p}<0.10)$ (see column labelled 'All Subjects' in Table 15, Table 16 and Table 17). 


\section{Anger \& Stress}

\subsection{Pre-Post Changes}

Trait anger, trait anger temperament, trait anger reaction, anger expression inwards, anger expression outwards and perceived stress demonstrated statistically significant decreases from pre to post intervention (See rows labelled 'All Subjects' in Table 18). Change in the proportion of subjects scoring in the $75^{\text {th }}$ percentile or greater for respective anger subscales was also evaluated. All anger measures, except for anger expression outwards, demonstrated statistically significant decreases from pre to post intervention. Anger expression outwards decreased by a non-significant amount (p-value=0.0654) (See rows labelled 'All Subjects' in Table 19). Overall, participants improved their psychosocial functioning for the primary psychosocial variables from pre to post intervention.

Among the exploratory psychosocial measures, anger control inwards and anger control outwards demonstrated statistically significantly increases from pre to post intervention while the anger expression index demonstrated a statistically significant decrease (See rows labelled 'All Subjects' in Table 18). Changes in the proportion of subjects scoring in the $75^{\text {th }}$ percentile or greater were also evaluated. There was a significant increase in the proportion of subjects scoring in the $75^{\text {th }}$ percentile or greater for anger control inwards and a significant decrease for the anger expression index. The proportion of subjects scoring in the $75^{\text {th }}$ percentile or greater for anger control outwards also increased, but by a non-significant amount (p-value=0.1185) (See rows labelled 'All Subjects' in Table 19). Overall, participants improved their psychosocial functioning for the exploratory psychosocial variables from pre to post intervention.

\subsection{Psychometric Properties}

Evaluations of baseline and post intervention PSS and STAXI-2 scores revealed high internal consistency for trait anger, trait anger temperament, trait anger reaction, anger expression inwards, perceived stress, anger control inwards and anger control outwards, except for anger expression outward which demonstrated slightly lower post intervention internal consistency $(\alpha$ $=0.62$ ) (Table 20). Sufficient internal consistency was determined using Nunnally \& Bernstein's suggested value of $0.70 .^{204}$ 


\section{Blood Pressure}

\subsection{Pre-Post Changes}

Twenty four hour and daytime systolic blood pressure demonstrated small but statistically significant decreases from pre to post intervention $(-1.89 \mathrm{mmHg}$ (SD 6.83), $\mathrm{p}$-value $=0.0124$ and $-2.12 \mathrm{mmHg}$ (SD 7.09), p-value $=0.0072$, respectively). Twenty four hour diastolic, daytime diastolic and nighttime systolic and diastolic blood pressure did not demonstrate statistically significant changes from pre to post intervention (See rows labelled 'All Subjects' in Table 21).

\subsection{Change Correlations with Psychosocial Measures}

There were no significant correlations between change in primary psychosocial variables (trait anger, trait anger temperament, trait anger reaction, anger expression inwards, anger expression outwards or perceived stress) and change in daytime or 24-hour blood pressure from pre to post intervention (See column labelled 'All Subjects' in Table 22 and Table 23). There were also no significant correlations with change in nighttime blood pressure (See column labelled 'All Subjects' in Table 24). Finally, there were no significant correlations between change in exploratory psychosocial variables (anger control inwards, anger control outwards and the anger expression index) and change in 24-hour, daytime and nighttime blood pressure from pre to post intervention (See column labelled 'All Subjects' in Table 25, Table 26 and Table 27).

\subsection{Multivariate Linear Regressions}

Results in this section have been organized in order of primary, secondary and exploratory hypotheses. The primary hypotheses testing the existence of an association between change in primary psychosocial variables (trait anger, trait anger temperament, trait anger reaction, anger

expression inwards, anger expression outwards and perceived stress) and change in daytime and 24-hour blood pressure from pre to post intervention have been summarized in Tables 28 to Table 41 . The secondary hypotheses testing the existence of an association between change in primary psychosocial variables and change in nighttime blood pressure from pre to post intervention have been summarized in Tables 42 to Table 48 . The exploratory hypotheses testing the existence of an association between change in exploratory psychosocial variables (anger control inwards, anger control outwards and the anger expression index) and change in daytime, 
24-hour and nighttime blood pressure from pre to post intervention have been summarized in Tables 49 to Table 57.

\subsubsection{Primary Hypotheses: Primary Psychosocial Measures \& 24-hour and Daytime Blood Pressure}

After controlling for baseline blood pressure and baseline psychometric score the full main effects multivariate regression models revealed that post intervention trait anger, trait anger temperament, trait anger reaction, anger expression inward, anger expression outward and perceived stress were not significantly associated with post intervention 24-hour or daytime systolic or diastolic blood pressure, respectively (For trait anger, see column labelled 'All Subjects' in Table 28 and Table 29. For trait anger temperament, see column labelled 'All Subjects' in Table 30, Table 31, Table 32 and Table 33. For trait anger reaction see column labelled 'All Subjects' in Table 34 and Table 35. For anger expression inward see column labelled 'All Subjects' in Table 36 and Table 37. For anger expression outward see column labelled 'All Subjects' in Table 38 and Table 39. For perceived stress see column labelled 'All Subjects' in Table 40 and Table 41).

\subsubsection{Secondary Hypotheses: Primary Psychosocial Measures \& Nighttime Blood Pressure}

After controlling for baseline nighttime blood pressure and baseline psychometric score the full main effects multivariate regression models revealed that post intervention trait anger, trait anger temperament, trait anger reaction, anger expression inward, anger expression outward and perceived stress were not significantly associated with post intervention nighttime systolic or diastolic blood pressure, respectively (For trait anger see column labelled 'All Subjects' in Table 42. For trait anger temperament see column labelled 'All Subjects' in Table 43 and Table 44. For trait anger reaction see column labelled 'All Subjects' in Table 45. For anger expression inward see column labelled 'All Subjects' in Table 46. For anger expression outward see column labelled 'All Subjects' in Table 47. For perceived stress see column labelled 'All Subjects' in Table 48). 


\subsubsection{Exploratory Hypotheses: Exploratory Psychosocial Measures \& 24-hour, Daytime and Nighttime Blood Pressure}

After controlling for baseline blood pressure and baseline psychometric score, the full main effects multivariate regression models revealed that post intervention anger expression inwards, anger expression outwards and the anger expression index were not significantly associated with post intervention 24-hour, daytime or nighttime systolic or diastolic blood pressure, respectively (For anger control inwards see column labelled 'All Subjects' in Table 49, Table 50 and Table 51. For anger control outwards see column labelled 'All Subjects' in Table 52, Table 53 and Table 54. For anger expression index see column labelled 'All Subjects' in Table 55, Table 56 and Table 57).

\subsection{Effect Sizes}

Beta coefficients of primary anger and stress predictor variables (trait anger, trait anger temperament, trait anger reaction, anger expressed in, anger expressed out and perceived stress) from the multivariate linear regression analyses were standardized to further examine their effect on 24-hour and daytime systolic and diastolic blood pressure. Cohen's guidelines the interpretation of standardized effects are $>0.50=$ large, $0.50-0.30=$ moderate, $0.30-0.10=$ small and $<0.10=$ insignificant. ${ }^{205}$ Based on these guidelines, standardized effects from the primary psychosocial predictor variables ranged from insignificant to small (Table 58 and Table $59)$.

\subsection{Post-Hoc Analyses}

Post-hoc analyses included evaluating changes in anthropomorphic measurements, drinking habits and exercise levels from pre to post intervention. This included evaluating whether changes in drinking habits or exercise levels had taken place since baseline (yes or no) as well as changes in total number of drinks consumed per week and total number of times exercised per week.

Thirteen percent of respondents reported a change in drinking habits and $41 \%$ reported a change in exercise habits since baseline. Paired t-tests revealed that the total average number of drinks consumed per week decreased and almost reached statistical significance ( $p$-value $=0.0528$ ). 
Drinking habits did not demonstrate statistically significant changes when treated as a categorical variable (i.e., drink > 10 drinks per week, yes or no). Vigorous and moderate exercise levels (only available as categorical variables) did not demonstrate statistically significant changes from pre to post intervention. One anthropomorphic measurement, waist size measured at the iliac crest, demonstrated a statistically significant decrease from pre to post intervention (pvalue $=0.0441)$. BMI also decreased but by a non-significant amount.

\section{Exploratory Sex-Based Analyses}

\subsection{Baseline Data}

Mean baseline 24-hour ambulatory blood pressure for males was 134/82 $\mathrm{mmHg}$ (SD 7.3/4.7) and for females was 135/82 (SD 8.3/6.3). Mean daytime ambulatory blood pressure for males was 140/87 mmHg (SD 7.6/5.4) and for females was 141/87 (SD 7.9/6.8). Mean baseline nighttime ambulatory blood pressure for males was $119 / 70 \mathrm{mmHg}$ (SD 9.6/7.2) and for females was 123/72 (SD 11.6/7.6). A summary of this information can be found in Table 60. There were no significant differences for any baseline variables between males and females except for waist circumference $(\mathrm{p}=0.0011)$, nighttime systolic blood pressure $(\mathrm{p}=0.0400)$, proportion of males vs. females who drank >10 drinks per week $(\mathrm{p}=0.0076)$ and proportion of males vs. females who used relaxation techniques regularly $(\mathrm{p}=0.0040)$.

Percentile ranks for anger scores indicate that the cohort was average in terms of anger levels. Perceived stress scores were also comparable to those observed with normative samples used to generate the PSS questionnaire. ${ }^{129}$ A summary of this information can be found in Table 61. Male and female study subjects demonstrated similar psychometric scores except for anger expression inwards $($ men $=18(\operatorname{SD} 4.5)$ and women $=16(\operatorname{SD} 4.3), \mathrm{p}$-value $=0.0231)$, anger control inwards $($ men $=20(\operatorname{SD} 5.4)$ and women $=23($ SD 5.6), $\mathrm{p}$-value $=0.0186)$ and the anger expression index $(\operatorname{men}=38(\mathrm{SD} 13.0)$ and women $=31(\mathrm{SD} 14.6), \mathrm{p}$-value $=0.0136)$.

\subsection{Baseline Correlations}

There were no significant correlations between baseline primary psychosocial variables (trait anger, trait anger temperament, trait anger reaction, anger expressed inwards, anger expressed outwards or perceived stress) and baseline 24-hour and daytime blood pressure among female subjects (See rows labelled 'Female' in Table 12 and Table 13). Among male subjects, one 
significant negative correlation emerged between baseline daytime systolic blood pressure and baseline anger expression inwards (p-value $=0.0428)($ See rows labelled 'Male' in Table 12 and Table 13). There were no significant correlations between baseline primary psychosocial variables and baseline nighttime blood pressure for either sex (See columns labelled 'Female' and 'Male' respectively in Table 14). Only baseline perceived stress approached statistical significance for a positive correlation with baseline nighttime systolic blood pressure among females $(\mathrm{p}<0.10)$.

There were no significant correlations between baseline exploratory psychosocial variables (anger control inwards, anger control outwards and the anger expression index) and baseline daytime, 24-hour and nighttime blood pressure among female subjects (See columns labelled 'Female' in Table 15, Table 16 and Table 17). Among male subjects, one significant negative correlation emerged between baseline anger expression index and baseline daytime systolic blood pressure $(\mathrm{p}=0.0492)$. One positive correlation approached statistical significance between baseline anger control inwards and baseline daytime systolic blood pressure $(\mathrm{p}<0.10)$ (See columns labelled 'Male' in Table 15, Table 16 and Table 17).

\section{Anger \&Stress}

\subsection{Pre-Post Changes}

All primary psychosocial variables (trait anger, trait anger temperament, anger expressed inwards, anger expressed outwards and perceived stress) except for trait anger reaction demonstrated statistically significant decreases from pre to post intervention among female subjects (See rows labelled 'Female' in Table 18). Among males, all primary psychosocial variables except for trait anger temperament and anger expression outwards demonstrated statistically significant decreases from pre to post intervention (See rows labelled 'Male' in Table 18). Changes in the proportion of subjects scoring in the $75^{\text {th }}$ percentile or greater from pre to post intervention were also evaluated. While decreases were observed for all primary psychosocial variables for both sexes, only females demonstrated statistically significant decreases in the proportion of subjects scoring in the $75^{\text {th }}$ percentile or greater for trait anger temperament and anger expression inwards (p-values $=0.0078$ and 0.0013 , respectively) (See rows labelled 'Female' and 'Male' Table 19). Overall, females appeared to improve on more primary psychosocial variables from pre to post intervention than males in this study. 
Exploratory psychosocial measures (anger control inwards, anger control outwards and the anger expression index) demonstrated similar patterns of improvement in psychosocial functioning from pre to post intervention. For both males and females, anger control inwards and anger control outwards increased by a statistically significantly amount from pre to post intervention and the anger expression index decreased by a statistically significant amount from pre to post intervention. The only exception was anger control outwards among male subjects which increased by a non-significant amount (p-value=0.1507) (See rows labelled 'Female' and 'Male' in Table 18). Again, changes in the proportion of subjects scoring in the $75^{\text {th }}$ percentile or greater from pre to post intervention were also evaluated. Among female subjects, a statistically significant increase was observed for anger control inwards $(\mathrm{p}=0.0225)$ while a statistically significant decrease was observed for the anger expression index $(\mathrm{p}=0.0010)$. Anger control outwards also increased but by a non-significant amount. The same trends were observed for male subjects but the changes did not reach statistical significance (See rows labelled 'Female' and 'Male' in Table 19). Overall, females appeared to improve on more exploratory psychosocial variables than males.

\section{Blood Pressure}

\subsection{Pre-Post Changes}

Among females, 24-hour systolic and daytime systolic and diastolic blood pressure all decreased significantly from pre to post intervention $(-3.09 \mathrm{mmHg}$ (SD 6.26), p-value=0.0006, -3.47 $\mathrm{mmHg}$ (SD 6.16), p-value=0.0001, -1.31 mmHg (SD 4.70), p-value=0.0436, respectively). Twenty four hour diastolic and nighttime systolic and diastolic blood pressure demonstrated nonsignificant decreases (-1.11 mmHg (SD 4.52), p-value=0.0741, -2.49 (SD 9.81), p-value=0.0652 and -0.42 (SD 5.65), p-value=0.5851 respectively) (See rows labelled 'Female' in Table 21). No change in any ambulatory blood pressure parameter was observed for male subjects (See rows labelled 'Male' in Table 21).

\subsection{Change Correlations with Psychosocial Measures}

Despite improvements in psychosocial functioning and modest reductions in blood pressure from pre to post intervention, there were no significant correlations between change in primary psychosocial variables (trait anger, trait anger temperament, trait anger reaction, anger 
expression inwards, anger expression outwards or perceived stress) and change in daytime or 24hour blood pressure from pre to post intervention for male or female subjects (See columns labelled 'Female' and 'Male' in Table 22 and Table 23). Among females, change in anger expression inwards approached statistical significance for positive correlations with daytime and 24-hour systolic blood pressure ( $\mathrm{p}$ 0.10). Similar non-significance was reported for correlations between change in primary psychosocial variables and change in nighttime blood pressure (See columns labelled 'Female' and 'Male' in Table 24).

Statistically significant correlations emerged after stratifying by sex between changes in exploratory psychosocial measures (anger control inwards, anger control outwards and the anger expression index) and changes in 24-hour, daytime and nighttime blood pressure from pre to post intervention. Among female subjects, there was a significant positive correlation between change in the anger expression index and change in daytime systolic blood pressure $(\mathrm{p}=0.0319)$ (See column labelled 'Female' in Table 26). Three additional correlations approached statistical significance $(\mathrm{p}<0.10)$ : a positive correlation between change in anger expression index and change in 24-hour systolic blood pressure (See column labelled 'Female' in Table 25), a negative correlation between change in anger control inwards and change in daytime systolic blood pressure (See column labelled 'Female' in Table 26) and a negative correlation between change in anger control outwards and change in daytime systolic blood pressure (See column labelled 'Female' in Table 26). Among male subjects, a significant positive correlation was found between change in anger control inwards and change in daytime systolic and diastolic blood pressure ( $\mathrm{p}=0.0351$ and 0.0330, respectively) (See column labelled 'Male' in Table 26).

\subsection{Multivariate Linear Regressions}

Results from this sex-based analysis are entirely exploratory. However, for ease of reporting they have been organized in the same order as the main multivariate linear regression results. More specifically they have been organized in the same order as the main results (primary, secondary and exploratory hypotheses). To review, the primary hypotheses testing the existence of an association between change in primary psychosocial variables (trait anger, trait anger temperament, trait anger reaction, anger expression inwards, anger expression outwards and perceived stress) and change in daytime and 24-hour blood pressure from pre to post intervention have been summarized in Tables 28 to Table 41 . The secondary hypotheses testing the existence 
of an association between change in primary psychosocial variables and change in nighttime blood pressure from pre to post intervention have been summarized in Tables 42 to Table 48. The exploratory hypotheses testing the existence of an association between change in exploratory psychosocial variables (anger control inwards, anger control outwards and the anger expression index) and change in daytime, 24-hour and nighttime blood pressure from pre to post intervention have been summarized in Tables 49 to Table 57.

\subsubsection{Primary Hypotheses: Primary Psychosocial Measures \& 24-hour and Daytime Blood Pressure}

After controlling for baseline blood pressure and baseline psychometric score, the full main effects multivariate regression models revealed that post intervention trait anger, trait anger reaction, anger expression inward and anger expression outward were not significantly associated with post intervention 24-hour or daytime systolic or diastolic blood pressure, respectively for either male and female subjects (For trait anger see columns labelled 'Female' and 'Male' respectively in Table 28 and Table 29. For trait anger reaction see columns labelled 'Female' and 'Male' respectively in Table 34 and Table 35. For anger expression inward see columns labelled 'Female' and 'Male' respectively in Table 36 and Table 37. For anger expression outward see columns labelled 'Female' and 'Male' respectively in Table 38 and Table 39)

However, post intervention trait anger temperament and perceived stress were found to be significantly associated with blood pressure after stratification by sex.

\section{Trait Anger Temperament}

Post intervention trait anger temperament was significantly positively associated with post intervention 24-hour systolic blood pressure for female subjects $(\mathrm{p}=0.01)$ (See column labelled 'Female' Table 30). More specifically, every one point increase in trait anger temperament scale was associated with a $2.71 \mathrm{mmHg}$ increase in 24-hour systolic blood pressure. A positive association with daytime systolic blood pressure approached statistical significance $(\mathrm{p}=0.07$, see column labelled 'Female' in Table 31). When trait anger temperament was reevaluated for exploratory purposes as a multilevel categorical predictor variable, after adjustment for baseline blood pressure and baseline trait anger temperament, post intervention trait anger temperament 
was still significantly associated with 24-hour systolic blood pressure (See column labelled 'Female' in Table 32). More specifically, being in the lowest quartile compared to the upper quartile of trait anger temperament scores after the MBSR program was associated with lower blood pressure $(\mathrm{p}=0.02)$, as was being in the second quartile compared to the upper quartile $(\mathrm{p}=0.03)$. Scoring in the third quartile compared to the upper quartile for post intervention trait anger temperament scores almost reached statistical significance $(\mathrm{p}=0.07)$. The same positive association approached statistical significance for trait anger temperament and daytime blood pressure (See column labelled 'Female' in Table 33). No association between trait anger temperament and 24-hour or daytime blood pressure was reported for male subjects (See column labelled 'Male' in Table 30, Table 31, Table 32 and Table 33).

\section{Perceived Stress}

After controlling for baseline blood pressure and baseline perceived stress score, the full main effects model revealed that post intervention perceived stress was significantly negatively associated with post intervention 24-hour systolic blood pressure among females $(p=0.03)$ (See column labelled 'Female' in Table 40). More specifically, every one point increase in perceived stress was associated with a $0.35 \mathrm{mmHg}$ decrease in 24-hour blood pressure. However, among male subjects, post intervention perceived stress score was significantly positively associated with post intervention 24-hour and daytime systolic blood pressure $(\mathrm{p}=0.02$ and $\mathrm{p}=0.02$, respectively) (See columns labelled 'Male' in Table 40 and Table 41). Every one point increase in perceived stress was associated with a $0.83 \mathrm{mmHg}$ or $0.91 \mathrm{mmHg}$ increase in 24-hour or daytime systolic blood pressure, respectively.

\subsubsection{Secondary Hypotheses: Primary Psychosocial Measures \& Nighttime Blood Pressure}

After controlling for baseline nighttime blood pressure and baseline psychometric score the full main effects multivariate regression models revealed that post intervention trait anger, trait anger temperament, trait anger reaction, anger expression inward and anger expression outward were not significantly associated with post intervention nighttime blood pressure in either sex (For trait anger see column labelled 'Female' and 'Male' in Table 42. For trait anger temperament see column labelled 'Female' and 'Male' in Table 43 and Table 44. For trait anger reaction see column labelled 'Female' and 'Male' in Table 45. For anger expression inward see column 
labelled 'Female' and 'Male' in Table 46. For anger expression outward see column labelled 'Female' and 'Male' in Table 47).

However, post intervention perceived stress was found to be significantly associated with post intervention nighttime systolic blood pressure among both males and females.

\section{Perceived Stress}

After controlling for baseline blood pressure and baseline perceived stress score, the full main effects model containing female subjects revealed that post intervention perceived stress was significantly negatively associated with post intervention nighttime systolic blood pressure $(\mathrm{p}=0.02)$ (See column labelled 'Female' in Table 48). More specifically, every one point increase in perceived stress was associated with a $0.52 \mathrm{mmHg}$ decrease in nighttime systolic blood pressure. Among male subjects, post intervention perceived stress was significantly positively associated with post intervention nighttime systolic blood pressure $(\mathrm{p}=0.04)$ (See column labelled 'Male' in Table 48). Every one point increase in perceived stress was associated with a $0.61 \mathrm{mmHg}$ increase in nighttime systolic blood pressure.

\subsubsection{Exploratory Hypotheses: Exploratory Psychosocial Measures \& 24-hour, Daytime and Nighttime Blood Pressure}

After controlling for baseline blood pressure and baseline psychometric score, the full main effects multivariate regression models revealed that post intervention exploratory variables (anger expression inwards, anger expression outwards and the anger expression index) were not significantly associated with post intervention 24-hour, daytime or nighttime blood pressure among males or females (For anger control inwards see column labelled 'Female' and 'Male' in Table 49, Table 50 and Table 51. For anger control outwards see column labelled 'Female' and 'Male' in Table 52, Table 53 and Table 54. For the anger expression index see column labelled 'Female' and 'Male' in Table 55, Table 56 and Table 57).

\subsection{Effect Sizes}

Beta coefficients for anger or stress predictor variables that emerged as statistically significant in the exploratory sex-based multivariate linear regression analyses were standardized to further 
examine effects on blood pressure. To review, Cohen's guidelines the interpretation of standardized effects are $>0.50=$ large, $0.50-0.30=$ moderate, $0.30-0.10=$ small and $<0.10=$

insignificant. ${ }^{205}$ Based on these guidelines, trait anger temperament was observed to have a large effect on 24-hour systolic blood pressure among females (standardized $\beta=0.62$ (SE 0.22), Table 68). Perceived stress had moderate effects on 24-hour and nighttime systolic blood pressure among females (standardized $\beta=-0.30$ (SE 0.13) and -0.36 (SE 0.14), respectively, Table 69). Among males, perceived stress had moderate effects on 24-hour and nighttime systolic blood pressure (standardized $\beta=0.49$ (SE 0.19) and 0.33 (SE 0.15), respectively, Table 70) and a large effect on daytime systolic blood pressure (standardized $\beta=0.52$ (SE 0.21), Table 70).

\section{All Results Summarized}

All measures of anger and perceived stress demonstrated statistically significant improvements from pre to post intervention when all subjects were examined together (Table 18 and Table 19). Blood pressure also demonstrated modest but statistically significant decreases from pre to post intervention for 24-hour and daytime systolic blood pressure when subjects were examined together (See column labelled 'All Subjects' in Table 21).

Despite the significant above-mentioned changes from pre to post intervention, baseline correlations and change correlations revealed no significant associations between both primary and exploratory psychometric measures and any ambulatory blood pressure parameter. The only exception to this was an exploratory anger measure (baseline anger control inwards) which demonstrated a positive correlation with baseline daytime systolic blood pressure (See column labelled 'All Subjects' in Table 16). After adjusting for baseline blood pressures, baseline psychometric values and other covariates, the multivariate linear regression analyses revealed that no primary psychosocial measure (post intervention trait anger, trait anger temperament, trait anger reaction, anger expression inwards, anger expression outwards and perceived stress) nor exploratory psychosocial measures (post intervention anger control inwards, anger control outwards and the anger expression index) were associated with post intervention 24-hour, daytime or nighttime systolic or diastolic blood pressure (See column labelled 'All Subjects' in Tables 62 - 67). Effect sizes as measured by standardized regression coefficients ranged from insignificant to small. 
The exploratory sex-based analysis revealed interesting points of significance. First, female participants improved on more psychometric measures than males in the study. Second, only female participants decreased blood pressure from pre to post intervention in the study, reducing their 24-hour and daytime systolic blood pressure by $-3.09 \mathrm{mmHg}$ and $-3.47 \mathrm{mmHg}$, respectively. Male participants either did not change or marginally raised their blood pressure from pre to post intervention in the study.

Exploratory baseline and change correlation analyses revealed some correlations of significance; however, most significant were findings from the follow-up multivariate linear regression analyses. After adjusting for baseline blood pressures and baseline psychometric values, among female subjects post intervention trait anger temperament was significantly positively associated with post intervention 24-hour systolic blood pressure among females while post intervention perceived stress was significantly negatively associated with post intervention 24-hour and nighttime systolic blood pressure. Among males, perceived stress was significantly positively associated with 24-hour, daytime and nighttime systolic blood pressure (See column labelled 'Female' and 'Male' in Table 62, Table 63, Table 64). Effect sizes as measured by standardized regression coefficients ranged from moderate to large. 


\section{Chapter 4 Discussion}

\section{General Overview}

Previous research has suggested an association between anger, perceived stress and blood pressure. These assertions have largely been based on cross-sectional studies that collected data at one time point that did not manipulate independent psychosocial variables of interest. More rigorous prospective longitudinal cohort research was proposed to test these relationships more directly thus add more robust evidence to the literature.

This thesis study was interested in using a prospective longitudinal cohort design to test the relationship between anger, perceived stress and blood pressure. This design was implemented by using a psychological therapeutic intervention to manipulate anger and perceived stress. Changes in anger and perceived stress were in turn evaluated for association with changes in ambulatory blood pressure. In the present study, mindfulness-based stress reduction (MBSR) was used as the tool to manipulate anger and perceived stress.

Findings from this study failed to demonstrate an association between change in anger or perceived stress with change in daytime, 24-hour or nighttime blood pressure after participants completed a standard 8-week MBSR program. Corresponding standardized regression coefficients indicated small to insignificant effects on blood pressure. Thus, these results failed to support the hypothesis of an association between change in anger and perceived stress with change in blood pressure after participants completed a standard 8-week MBSR program. Exploratory analyses revealed significant associations after stratification by sex.

This study has helped to clarify the anger, perceived stress and blood pressure literature by producing results that have been based on a stronger study design. More specifically, this study has contributed results that have been based on a prospective longitudinal cohort design where an intervention was used to manipulate the independent psychosocial variables of interest. By comparison, the majority of anger, perceived stress and blood pressure literature has been based on single time point cross-sectional observational studies that did not manipulate independent psychosocial predictor variables of interest. Based on a stronger study design, the results of this 
thesis have helped to clarify the literature and our understanding of anger and perceived stress as suspected risk factors for hypertension development.

\section{Findings, Explanations and Implications}

\subsection{Primary Findings}

To review, the primary objective of this study was to establish the existence of an association between change in trait anger, trait anger temperament, trait anger reaction, anger expression inward, anger expression outward, perceived stress and change in 24-hour and daytime blood pressure among unmedicated stage-1 hypertensive subjects who completed a standard 8-week MBSR protocol. Secondary objectives included establishing associations between changes in these primary psychosocial variables and changes in nighttime blood pressure. Exploratory analyses included evaluating the existence of an association between change in anger control inwards, anger control outwards and the anger expression index with change in 24-hour, daytime and nighttime blood pressure. Additional exploratory analyses included evaluating the existence of any sex differences.

Participants enrolled in the study were physically healthy with the exception of elevated blood pressure. Baseline psychosocial data indicated that the group was average in terms of their anger profiles based on percentile rank information from the STAXI-2 professional manual.

Comparing mean baseline PSS scores from this study to the normative samples used during PSS validation in 1983, the mean score in this study was almost identical to scores obtained for the normative samples (normative sample $1=23$ (SD 7.3), normative sample $2=24$ (7.8), PSS average in this study $=24(8.2)) .{ }^{129}$ Compared to a separate probability sample collected in 1983 in the United States, the mean PSS score from this study was only slightly above average $($ probability sample mean $=20(\mathrm{SD} 7.5)) .{ }^{187}$ Based on this information it can be concluded that participants in this study were overall physically and mentally healthy.

Taking a collective and comprehensive approach to reviewing the results reveals that subjects benefitted psychologically (improved anger and stress) and physiologically (reduced blood pressure) from enrolling in MBSR. All measures of anger and perceived stress demonstrated statistically significant improvements from pre to post intervention. For anger, this was maintained when anger constructs were evaluated both as raw scores and clinically relevant 
binary variables dividing scores between the top $25 \%$ percentile and bottom $75 \%$ percentile (Table 18 and Table 19). The only exceptions were anger expression outwards and anger control outwards when evaluated as binary variables. However, both variables still demonstrated changes in the direction of therapeutic benefit that approached statistical significance ( $\mathrm{p}=0.0654$ and $\mathrm{p}=0.1185$, respectively). In addition to psychosocial functioning, 24-hour and daytime systolic blood pressure demonstrated statistically significant decreases from pre to post intervention. Taken together, this information outlines improved psychosocial and physiological profiles from pre to post intervention for the subjects enrolled in this study.

But despite these significant pre-post changes, baseline correlation analyses and pre-post change correlation analyses revealed no significant correlations between anger, perceived stress and any ambulatory blood pressure parameter. The only exception was a significant positive baseline correlation between an exploratory variable (anger control inwards) and daytime systolic blood pressure. However, after adjusting for baseline blood pressures, baseline psychometric values as well as other covariates, the multivariate linear regression analyses revealed that post intervention anger and perceived stress were not associated with post intervention 24-hour, daytime or nighttime blood pressure. In summary, pre-post data illustrated statistically significant improvements in psychosocial functioning and blood pressure. However, these variables failed to demonstrate association with one another in follow-up correlation and multivariate linear regression analyses.

Together these results highlight a significant feature: subjects improved anger and stress, as well as modestly improved their blood pressure. However, these changes were unassociated with one another in this study.

\section{Excluded Explanations}

A number of possible explanations for these findings can be considered unlikely and thus excluded. First and foremost is the possibility that predictor variables did not change sufficiently from pre to post intervention (i.e., that MBSR did not manipulate anger or stress by a sufficient amount to detect an association with blood pressure). Raw pre-post data suggests this is not the case. Statistically significant reductions for trait anger, trait anger temperament, anger expression inward, anger expression outward, perceived stress and the anger expression index were observed from pre to post intervention for all subjects. Correspondingly, there were statistically 
significant increases in anger control inward and anger control outward. According to Spielberger "the development of internal controls over the experience and expression of anger is generally seen in a positive light" particularly if they are accompanied by an improvement in anger expression. Thus, these reciprocal changes in anger expression (which was reduced) and anger control (which was improved) align with a therapeutic improvement in subjects' anger profile.

Further evidence of improved psychosocial functioning included statistically significant improvements in the proportion of subjects scoring in the $75^{\text {th }}$ percentile or greater on all of the anger subscales except for anger control outwards and anger expression outwards. Although anger control outwards and anger expression outwards did not demonstrate statistically significant changes, these two variables still demonstrated improvement trending in the direction of therapeutic benefit. According to Spielberger, anger scores in the $75^{\text {th }}$ percentile greater "have been shown to be associated with elevated blood pressure and hypertension". 2, 57, 66, 198 Considering this information, the improvement observed in the proportion of subjects scoring in the 75th percentile or greater is clinically significant and reinforces raw score data outlining that participants became less angry from pre to post intervention in this study. Taken together this information excludes the possible explanation that a lack of association in this study was due to lack of psychosocial improvement.

Measurement error and methodological inconsistencies are also unlikely explanations for this study's findings. This study was prospective and was rigorously executed, using only validated psychometric questionnaires and the gold standard in blood pressure measurement. While measurement error can never be entirely eliminated, poor methodology is an unlikely explanation for the non-significance reported in this study.

Lack of participant engagement is also an unlikely explanation for this study's findings. The HARMONY trial was advertised as a non-pharmacological treatment intervention for blood pressure lowering for individuals with untreated stage-1 hypertension. Anecdotally, many participants in the study had been informed of their elevated blood pressure by a health care provider and that pharmacological treatment may be required in the future. Many subjects enrolled in the HARMONY study were thus highly motivated to find non-drug alternatives for blood pressure lowering. 
Evidence of this motivation can be demonstrated by adherence to the MBSR program. This was quantified by the number of minutes spent per day on homework meditation practice. The average amount of homework completed was 32 minutes ( \pm 15 minutes) out of the required 45 minutes per day; approximately $71 \%$. Adherence or 'acceptable compliance' has been defined by pharmacological anti-hypertensive literature as taking $\geq 80 \%$ of prescribed antihypertensive medications. ${ }^{206,207}$ Using this guideline, acceptable compliance to the MBSR program would be 45 minutes $\mathrm{x} 80 \%=36$ minutes. Considering that average adherence to pharmacological therapy is estimated to be $50 \%-70 \%{ }^{208}$ adherence in the MBSR program was on par, if not slightly higher than the average adherence to pharmacological therapy. Moreover, some items on the homework logs did not measure number of minutes of homework practiced but still represented some form of mindfulness meditation. Therefore, this MBSR adherence estimate is likely a conservative estimate, with more participants engaging in the daily homework meditation practices than captured by homework logs. Taken together, it can be concluded that lack of participant engagement in the MBSR program is an unlikely explanation for the non-significant findings reported between stress, anger and blood pressure in this study.

To summarize, there are a number of explanations for these findings that are unlikely and can therefore be excluded: lack statistically significant improvement in the predictor variables (psychosocial functioning), poor measurement methodology and lack of participant engagement/low treatment adherence. The exclusion of these variables has been supported by evidence from the study.

\section{Potential Explanations}

In this study perceived stress, anger and blood pressure decreased by statistically significant amounts from pre to post intervention. However, these decreases failed to demonstrate an association with one another. One hypothesis to explain these findings may be that the relationship between anger, perceived stress and blood pressure is mediated by a third variable.

The existence of a third variable mediating the relationship between psychosocial variables and blood pressure could exist. Evidence from one study provides strong support for this hypothesis. ${ }^{209}$ In this study, information was collected on job stress, blood pressure, as well as adaptive and maladaptive stress coping behaviours. The authors found no association between work stress and blood pressure. However, the authors did find that "the ways [in which] 
individuals reported coping with stress were significantly related to blood pressure, with blood pressure elevation effects appearing to be mediated largely by dietary and drinking habits and physical inactivity". ${ }^{209}$ In another separate study, improved stress management was individually associated with improved hypertensive risk factors, such as weight. ${ }^{210}$ Taken together, this evidence outlines a possible model by which improved psychosocial functioning and blood pressure could be indirectly associated via health behaviours or stress coping mechanisms.

Post-hoc analyses of changes in anthropomorphic measurements, drinking habits and exercise levels from pre to post intervention revealed some support for this model. Indications of lifestyle and health behaviours known to be linked to blood pressure, specifically waist size ${ }^{211}$ and alcohol consumption, ${ }^{212-214}$ improved from pre to post intervention in this study. Based on the evidence that individuals respond to stress with different stress coping mechanisms that are linked to blood pressure (such as drinking habits and exercise ${ }^{209}$ ) and that stress management has been individually associated with improved hypertensive risk factors ${ }^{210}$ suggests that improved health behaviours/stress coping mechanisms may be the mechanism linking the association between psychosocial variables and blood pressure in this study. It should be mentioned that the post-hoc analysis does not claim anything other than indications of improved lifestyle. However, results from this analysis do align with a possible model whereby improved psychosocial functioning and hypertension are indirectly associated with one another via health behaviours or stress coping mechanisms. This model offers one possible explanation for the overall findings of this study.

Given that this was a neutral study other possible explanations for these findings exist. First is the possibility that baseline anger and perceived stress were too low to demonstrate an association with blood pressure. As stated earlier, high anger scores particularly those $\geq 75^{\text {th }}$ percentile "have been shown to be associated with elevated blood pressure and hypertension"., 57, 66, 198 This raises the possibility that associations between psychosocial variables and blood pressure may exist among those who suffer from severe anger or stress issues. If true, this could explain the lack of association observed in this thesis study. Future directions should include evaluating associations between stress, anger and blood pressure among those who suffer from severe stress or anger issues. 
Another possible explanation for these results includes the narrow racial/ethnic profile of participants in this study. Eighty two percent of participants in this study self-identified as Caucasian. As reviewed in the introduction, the existence of underlying racial/ethnic effects is still unclear; thus it is possible that different findings could emerge when studying participants from different racial/ethnic backgrounds.

Thus to summarize, a number of explanations exist that could explain the study's findings. First is the existence of a mediating variable such as a health related behaviour or stress coping mechanism that indirectly links psychosocial variables and blood pressure. Post-hoc analyses support this possible hypothesis. Other explanations include that associations between anger, perceived stress and blood pressure may only exist among those suffering from severe anger or stress issues. A final possible explanation includes that the relationship between psychosocial variables and blood pressure among participants of different racial/ethnic backgrounds. This list of possible explanations is by no means exhaustive. If contemplated, any future research should focus on these unexplored areas to clarify these possible explanations.

\section{Implications}

To the best of our knowledge, this is the first study to use a prospective longitudinal cohort design to test the relationships between perceived stress, anger and blood pressure by using validated questionnaires, a gold standard blood pressure measurement technique and a tool to manipulate the psychosocial predictor variables of interest. The results from this study have added clarity to the field by contributing findings that are rooted in a stronger study design than has been previously reported by the majority of the literature.

As covered in the first chapter, the literature was equivocal (but not entirely) in terms of an association between anger and blood pressure. The literature was more positive with respect to an association between perceived stress and blood pressure. Reasons for a divide in the anger literature are unclear, but proposed explanations include differences in race/ethnicity, sex/gender and varying blood pressure measurement techniques. Differing selection of covariates between studies has also been proposed as an explanation for varying findings. ${ }^{24}$ With respect to perceived stress, the majority of the literature reporting a positive association was based on nonvalidated measures of perceived stress. These divides in the literature, poor study designs and lack of robust measurement tools was the impetus behind this study. 
Results from this study failed to demonstrate an association between change in anger and perceived stress and change in ambulatory blood pressure among stage- 1 untreated hypertensive subjects who underwent an 8-week mindfulness based stress reduction program. The results from this study conform to approximately half of the anger and blood pressure literature and less than half of the perceived stress literature suggesting no association between these variables and blood pressure.

The conclusions of this thesis have a number of implications on the literature. Most important is the failure to support the hypothesis of an association between anger, perceived stress and blood pressure in a field of research that is highly divided on the issue. The implications of this rejection are particularly significant due to the robustness of the evidence provided by this study, which was achieved through the use of a stronger study design than has been previously reported by the majority of the literature

However, what has also emerged from this work is the possibility that a different model may link these variables. Psychosocial functioning and blood pressure improved from pre to post intervention but failed to demonstrate association with one another in the correlation and multivariate regression analyses. Based on this trend, it is possible that these variables are linked indirectly by another variable such as stress coping mechanisms or health behaviours. Evidence from the literature combined with results from the post-hoc analyses preformed in this study support this proposed model. This potential explanation has its own implications on the literature, particularly with regards to changing our current understanding of the relationship between psychosocial variables and blood pressure.

In summary, the implications of this study on the literature include clarifying our understanding of anger and perceived stress as suspected risk factors for hypertension, as well as a call for new research that focus on evaluating indirect associations between anger, perceived stress and blood pressure. More specifically, research should focus on the effects of stress/psychosocially driven coping mechanisms/health behaviours and their relationship to blood pressure and hypertension.

\subsection{Exploratory Sex-Based Findings}

The exploratory sex-based analysis revealed several interesting points of significance. First, female participants improved on more psychometric measures compared to male counterparts in 
the study. Second, only female participants had a fall in blood pressure from pre to post intervention in the study. Female participant's 24-hour and daytime systolic blood pressure fell by $-3.09 \mathrm{mmHg}$ and $-3.47 \mathrm{mmHg}$, respectively, while male participants increased their 24-hour and daytime systolic blood pressure by $0.30 \mathrm{mmHg}$ and $0.37 \mathrm{mmHg}$, respectively.

Baseline and change correlation analyses revealed some correlations of significance. However, follow-up multivariate linear regression analyses revealed specific associations of significance. After adjusting for baseline blood pressures and baseline psychometric values, post intervention trait anger temperament was found to be significantly positively associated with post intervention 24-hour systolic blood pressure among females (See column labelled 'Female' in Table 30 and Table 32), and post intervention perceived stress was negatively associated with post intervention 24-hour and nighttime systolic blood pressure among females and positively associated with 24-hour, daytime and nighttime systolic blood pressure among males (See column labelled 'Female' and 'Male' in Table 40, Table 41, Table 48).

These findings outline an interesting interaction between sex/gender, anger, perceived stress and blood pressure. It should be noted that this finding is exploratory and thus only hypothesis generating. Therefore, the following discussion regarding these findings is entirely speculative.

\section{Trait Anger Temperament}

Trait anger temperament measures the disposition to experience anger without specific provocation. In this study, higher trait anger temperament scores after the intervention were associated with higher 24-hour systolic blood pressure for female subjects. Every one point increase in trait anger temperament after the MBSR program (from total score range of 4 to 16 points) corresponded to an increase of $2.71 \mathrm{mmHg}$ for 24-hour systolic post-intervention blood pressure.

This finding aligns with the study's raw psychometric data where only females demonstrated statistically significant reductions in trait anger temperament from pre to post intervention. The literature review on trait anger did outline possible sex differences suggesting a stronger effect among females. ${ }^{67,69,73-75}$ Additional evidence also suggests that males and females respond differently to stress reduction interventions. ${ }^{215}$ Females have been cited to derive greater benefit from stress reduction, ${ }^{216}$ more often reporting cognitive benefits over physical/athletic benefits 
reported by males. ${ }^{217,218}$ Studies using MBSR to treat hostility among inmates have reported greater pre-post improvements on self-report measures of hostility among female subjects. ${ }^{219}$ Thus, this improved trait anger temperament finding among female subjects aligns with what is reported in the literature.

Only a handful of studies have examined trait anger temperament and blood pressure. ${ }^{55,56,66}$ These have been limited to studies on male adolescents or male war veterans and thus are limited in generalizability. Therefore this analysis of trait anger temperament is a novel contribution to the field.

\section{Perceived Stress}

The results for perceived stress reveal conflicting findings. Perceived stress was negatively associated with 24-hour and nighttime systolic blood pressure among females and positively associated with 24-hour, daytime and nighttime systolic blood pressure among males in the multivariate linear regression analyses.

The findings for the female participants are in opposition to all of the perceived stress literature which has only reported either no association or a positive association between perceived stress and blood pressure. Several steps were taken to evaluate the validity of these findings. A literature review was performed to determine whether stress reduction interventions had previously increased stress among women. No support could be found for this hypothesis. A review of the original data revealed no errors or outliers and reexamining the correlation matrices between post intervention perceived stress and post intervention blood pressure revealed no discernible pattern in the data (Figure 1 and Figure 2). Based on the contrast of this finding to the otherwise psychological and physiological beneficial outcomes observed for females in this study, the results of the literature review, the re-examination of the raw data and the high number of comparison procedures undertaken in these analyses it was determined that this result was most likely a chance finding.

Among males, results from the exploratory sex-based analysis revealed a positive association between perceived stress and blood pressure. This finding is consistent with approximately half of the literature that has studied perceived stress as measured by a validated questionnaire and blood pressure (Table 6). These results conform to an even greater number of studies from the 
non-validated perceived stress literature (Table 6). However, the exploratory sex-based analysis also revealed that male participants did not change their blood pressure in this study. Thus, the validity of these results from the multivariate linear regression analyses for male subjects is questionable. Due to the lack of blood pressure lowering and high number of comparison procedures undertaken in these analyses it was determined that the perceived stress results for male subjects were also likely chance findings.

\section{Exploratory Sex-Based Analyses: Summary}

Findings from this exploratory sex-based analysis revealed a positive association between trait anger temperament and 24-hour systolic blood pressure among female subjects. For reasons discussed above, results from the perceived stress analyses were determined to likely be chance findings.

In terms of reconciling these findings with the proposed model of an indirect association between psychosocial variables and blood pressure linked via stress coping mechanisms/health behaviours, the exploratory nature of the sex-based analysis is limited in its scope of impact. More research on an adequately sized female sample must be performed in order to determine both the existence and validity of the association between trait anger temperament and blood pressure. It should be noted that no other anger construct was linked to blood pressure for males or females. Thus, this single finding does not significantly challenge the earlier proposed model of an indirect association between anger, perceived stress and blood pressure.

In fact, the lack of effect observed among the remaining anger constructs, despite the greater psychosocial improvement and blood pressure lowering observed among females, reinforces the proposed hypothesis of indirect association between psychosocial functioning and blood pressure. Interestingly, data from the Canadian National Population Health Survey which studied gender differences in health found that "social structural and psychosocial determinants of health were more important for females" 220 over behavioural determinants. Sociological models such as the 'tend and befriend' stress response demonstrated by females and the 'fight or flight' stress response demonstrated by males ${ }^{221}$ further underscores the possibility MBSR provided female subjects with stress coping mechanisms that better matched their stress behaviours compared to male subjects. Taken together, this information aligns directly with a hypothesis that an 
intervention like MBSR could have a greater effect among females by possibly improving stress coping mechanisms and health behaviours linked to blood pressure.

In summary, findings from this exploratory sex-based analysis revealed a positive association between trait anger temperament and 24-hour systolic blood pressure among females. However, the exploratory nature of this analysis renders these findings hypothesis generating and explanations entirely speculative. The increased risk of type 1 error due to many comparison procedures challenges the strength of this finding. Therefore, while exciting, these results must be interpreted appropriately and confirmed with follow up research.

\section{Limitations and Considerations}

\section{Study Design}

The prospective longitudinal cohort within-group design is a limitation to this study. While this study was entirely neutral reporting no association between change in anger, perceived stress and blood pressure, only a randomized controlled trial design can best account for confounding and provide the most robust explanations for the study's findings. Moreover, despite the inclusion of baseline blood pressures and psychometric values in all regression analyses, without a randomly allocated placebo control group statistical measurement phenomena such as regression to the mean may also be exacerbated. ${ }^{222}$ Thus, the prospective longitudinal cohort within-group design used in this thesis carries some inherent limitations that must be considered when evaluating the results of this study.

\section{Participant Homogeneity}

Subjects were recruited from the Toronto and greater Toronto area using advertising in the Toronto Star (online but majority in print), posters in Toronto General Hospital and Sunnybrook Health Sciences Centre and on a referral basis from physicians. A significant proportion of the participants learned about study through printed advertisements in the Toronto Star. Due to funding restraints and participant travel considerations, only select areas of Toronto received the printed advertisements. This recruitment strategy invariably selected individuals with a certain level of reading ability and income, thus potentially influencing the demographic of individuals 
enrolled in study. It may also have impacted the racial/ethnic profile of the participants enrolled. Together this limits the generalizability of this study's findings.

\section{Participant Motivation}

Participants in this study were originally enrolled into the HARMONY trial, a study interested in evaluating a non-pharmacological treatment option for blood pressure lowering among untreated stage-1 hypertensives. Many participants in the trial had been informed by a health care provider that they may need pharmacotherapy in the future. Participants enrolling in the study were highly motivated to find non-drug alternatives for blood pressure lowering, as evidenced by their adherence to the MBSR program discussed earlier. These participants may not be an accurate reflection of the general population therefore also limiting the generalizability of these findings.

\section{Biased Analyses}

The analysis implemented in this study used complete pre-post datasets. Those with missing data were excluded. This approach inherently created a biased sample as only those participants who completed all required study visits were included in the analyses. Multiple imputation is one option to manage missing data; however, it is complex and computationally intensive. ${ }^{223}$ The $^{2}$ decision to use multiple imputation is based in part on why data are missing. In this study, the missing data was not missing at random. More specifically, differences between subjects who completed all visits and subjects who did not complete all visits could not be explained by the same reason (i.e., a broken blood pressure monitor). Reasons for missing data are unclear, but may include participant responses to the intervention (i.e., some participants may not have liked the program) as well as changes in personal commitments that restricted their participation in the study. When data is not missing at random, multiple imputation carries a risk of introducing its

own bias into a sample. ${ }^{223}$ Thus, based on both complexity of imputation and type of missing data in this study, multiple imputation was not used.

\section{Future Directions}

\section{Evaluation of Indirect Mechanisms}

Future directions should include evaluating the proposed model linking psychosocial health with blood pressure via stress coping mechanisms and health behaviours. More specifically, future 
directions should involve evaluating the effectiveness of programs that target improved psychosocial health in addition to heart healthy behavioural changes with the goal of evaluating blood pressure change or control. Using ambulatory blood pressure as an outcome, one type of future research study could use a randomized controlled trial format where subjects could be randomized to lifestyle recommendations (usual care) or lifestyle recommendations plus MBSR. This type of study would enable a better understanding of the existence and extent of association between psychosocial health, heart healthy behaviours and blood pressure. If a linkage was confirmed, and specific psychosocial features could be identified that would make some patients more or less adherent to heart healthy behaviours linked to blood pressure, then it would be possible to refer (or even develop) psychosocial programs that may assist patients with adhering to lifestyle changes.

Preliminary research in this area has already begun among coronary heart disease patients. Using a randomized controlled trial format, cognitive behavioural therapy was demonstrated to decrease the rate of fatal and non-fatal first recurrent cardiovascular events as well as the number of acute myocardial infarctions. ${ }^{224}$ Although not examining blood pressure directly, this study underscores the strong potential existence of an indirect linkage between psychosocial health and blood pressure, and the need for future research to better understand the interplay of this relationship as it is mediated by stress coping mechanisms and health behaviours.

\section{Angry/Stressed Populations}

The participants examined in this study were healthy middle-aged adults with the exception of mildly elevated blood pressure. Based on anger literature suggesting that those scoring in the $75^{\text {th }}$ percentile or greater have elevated blood pressure ${ }^{57,66,198}$ and that those scoring in the $90^{\text {th }}$ percentile or greater have an increased risk for coronary artery disease and heart attacks, ${ }^{197}$ it would be of interest to examine associations between perceived stress, anger and blood pressure among those suffering from more severe stress or anger issues. Thus, future directions should also include evaluating the linkages between psychosocial health, stress coping mechanisms/health behaviours and hypertension among those with more severe anger or stress issues.

\section{Different Racial/Ethnic Populations}


Majority of participants in this study self-identified as Caucasian (82\%). As explained in the introduction, racial/ethnic contributions are still unclear; thus, different results may have been obtained had this study been completed on subjects from different racial/ethnic backgrounds. Therefore, future research should evaluate direct associations between anger, perceived stress and blood pressure, as well as indirect associations via stress coping mechanisms and health behaviours among subjects from different racial/ethnic backgrounds.

\section{Physiological Measures of Stress}

Psychosocial testing provides valuable subjective accounts of psychosocial functioning. However, objective physiological measures of stress (such as cortisol or inflammatory markers) could enable a better understanding the relationship between psychosocial variables and blood pressure. Although no direct associations between anger, perceived stress and blood pressure were identified in this study, any future research studying indirect linkages, more stressed/angry populations and other racial/ethnic groups would be bolstered by the addition of objective physiological stress measures. Thus, future research in this area should consider the inclusion of external physiological measures of stress.

\section{Sex/Gender Based Analyses}

The results of this study have underscored the critical need for more sex-based analysis in the area of psychosocial risk factors, health behaviours and blood pressure. Future directions must focus on including and studying males and females (together and separately) to address the existence of possible underlying sex differences.

\section{Concluding Remarks}

Previous research suggested an association between anger, perceived stress and blood pressure. These assertions were based almost exclusively on cross-sectional data that collected information at one time point and did not manipulate independent psychosocial variables of interest. Using a prospective longitudinal cohort within-group design with an intervention designed to affect the predictor variables of anger and perceived stress, findings from this study failed to demonstrate that change in anger and perceived stress were associated with change in ambulatory blood pressure among untreated stage-1 hypertensive subjects who completed an 8-week mindfulness- 
based stress reduction program. Through the use of a stronger study design, this thesis has provided more robust evidence to a divided field and contributed to a better understanding of anger and perceived stress as suspected risk factors for hypertension development.

Anger, perceived stress and blood pressure improved from pre to post intervention but failed to demonstrate association with one another in the correlation and regression analyses. These results, combined with post-hoc analysis of study data and evidence from the literature, suggest a possible model whereby psychosocial variables are indirectly linked to blood pressure through stress coping mechanisms and health behaviours. Future efforts should focus on studying the effects of psychosocially driven stress coping mechanisms and health behaviours and their relationship to blood pressure and hypertension. 


\section{Tables}

Table 1. Studies examining state anger and blood pressure

\begin{tabular}{|c|c|c|c|c|c|c|c|}
\hline $\begin{array}{l}\text { Author } \\
\text { (year) }\end{array}$ & Study type & Population & $\begin{array}{l}\% \text { identify } \\
\text { as non- } \\
\text { Caucasian }\end{array}$ & $\begin{array}{l}\text { Sample } \\
\text { size } \\
\% \text { male }\end{array}$ & $\begin{array}{l}\text { Anger } \\
\text { assessment } \\
\text { tool }\end{array}$ & BPM type & Findings \\
\hline $\begin{array}{l}\text { Schneider } \\
(1986)^{58}\end{array}$ & $\begin{array}{l}\text { Cross- } \\
\text { sectional }\end{array}$ & $\begin{array}{l}\text { HTN \& WCH, } \\
\text { undergraduate } \\
\text { students }\end{array}$ & $10 \%$ & $\begin{array}{l}33 \\
91 \% \text { male }\end{array}$ & STAXI & $\begin{array}{l}\text { Automated } \\
\text { home }\end{array}$ & No difference between groups \\
\hline $\begin{array}{l}\text { Friedman } \\
(2001)^{99}\end{array}$ & $\begin{array}{l}\text { Cross- } \\
\text { sectional }\end{array}$ & $\begin{array}{l}\text { Mild HTN \& } \\
\text { NTN, middle } \\
\text { aged adults }\end{array}$ & $14 \%$ & $\begin{array}{l}283 \\
100 \% \text { male }\end{array}$ & STAXI & $\mathrm{ABPM}$ & No difference between groups \\
\hline $\begin{array}{l}\text { Johnson } \\
(1984)^{57}\end{array}$ & $\begin{array}{l}\text { Cross- } \\
\text { sectional }\end{array}$ & $\begin{array}{l}\text { NTN, high } \\
\text { school students }\end{array}$ & $42 \%$ & $\begin{array}{l}1060 \\
57 \% \text { male }\end{array}$ & STAXI & $\begin{array}{l}\text { Automated } \\
\text { office }\end{array}$ & No association \\
\hline \multicolumn{8}{|c|}{$\begin{array}{l}\text { Acronyms: Blood pressure (BP), Blood pressure measurement (BPM), hypertension (HTN), white coat hypertension }(\mathrm{WCH}), \\
\text { normotension (NTN), State-trait Anger Expression Inventory (STAXI), ambulatory blood pressure monitoring (ABPM), Harburg Anger- } \\
\text { In/Anger-Out Scale (HAX) }\end{array}$} \\
\hline
\end{tabular}


Table 2. Studies examining trait anger and blood pressure

\begin{tabular}{|c|c|c|c|c|c|c|c|}
\hline $\begin{array}{l}\text { Author } \\
\text { (year) }\end{array}$ & Study type & Population & $\begin{array}{l}\% \text { identify as } \\
\text { non- } \\
\text { Caucasian }\end{array}$ & $\begin{array}{l}\text { Sample } \\
\text { size } \\
\% \text { male }\end{array}$ & $\begin{array}{l}\text { Anger } \\
\text { assess- } \\
\text { ment tool } \\
\end{array}$ & BPM type & Findings \\
\hline $\begin{array}{l}\text { Schneider } \\
(1986)^{58}\end{array}$ & Cross-sectional & $\begin{array}{l}\text { HTN \& WCH, } \\
\text { undergraduate } \\
\text { students }\end{array}$ & $10 \%$ & $\begin{array}{l}33 \\
91 \% \text { male }\end{array}$ & STAXI & Automated home & $\begin{array}{l}\text { No difference } \\
\text { between groups }\end{array}$ \\
\hline $\begin{array}{l}\text { Friedman } \\
(2001)^{99}\end{array}$ & Cross-sectional & $\begin{array}{l}\text { Mild HTN \& } \\
\text { NTN, middle } \\
\text { aged adults }\end{array}$ & $14 \%$ & $\begin{array}{l}283 \\
100 \% \\
\text { male }\end{array}$ & STAXI & ABPM & $\begin{array}{l}\text { No difference } \\
\text { between groups }\end{array}$ \\
\hline $\begin{array}{l}\text { Lerman } \\
(1989)^{72}\end{array}$ & Cross-sectional & $\begin{array}{l}\mathrm{HTN} \& \mathrm{WCH} \\
\text { middle aged } \\
\text { adults }\end{array}$ & $25 \%$ & $\begin{array}{l}98 \\
57 \% \text { male }\end{array}$ & STAXI & $\begin{array}{l}\text { Non-standardized } \\
\text { manual \& ABPM }\end{array}$ & $\begin{array}{l}\text { Trait anger more } \\
\text { common among } \\
\text { hypertensives }\end{array}$ \\
\hline $\begin{array}{l}\text { Durel } \\
(1989)^{73}\end{array}$ & Cross-sectional & $\begin{array}{l}\text { NTN \& } \\
\text { unmedicated mild } \\
\text { HTN, middle } \\
\text { aged adults }\end{array}$ & $53 \%$ & $\begin{array}{l}135 \\
60 \% \text { male }\end{array}$ & STAXI & $\begin{array}{l}\text { ABPM \& } \\
\text { Automated office }\end{array}$ & $\begin{array}{l}\text { Positive } \\
\text { association } \\
\text { (females only) }\end{array}$ \\
\hline $\begin{array}{l}\text { Webb } \\
(2002)^{110}\end{array}$ & Cross-sectional & $\begin{array}{l}\text { NTN, medicated } \\
\text { HTN \& } \\
\text { unmedicated } \\
\text { HTN, middle } \\
\text { aged adults }\end{array}$ & $100 \%$ & $\begin{array}{l}90 \\
0 \% \text { male }\end{array}$ & STAXI & Automated office & $\begin{array}{l}\text { No difference } \\
\text { between groups }\end{array}$ \\
\hline $\begin{array}{l}\text { Laude } \\
(1997)^{68}\end{array}$ & Cross-sectional & $\begin{array}{l}\text { NTN \& mild } \\
\text { HTN, middle } \\
\text { aged adults }\end{array}$ & $0 \%$ & $\begin{array}{l}25 \\
20 \% \text { male }\end{array}$ & STAXI & $\begin{array}{l}\text { Continuous finger } \\
\text { BP }\end{array}$ & $\begin{array}{l}\text { High trait anger } \\
\text { subjects } \\
\text { demonstrated } \\
\text { significantly } \\
\text { higher BP }\end{array}$ \\
\hline
\end{tabular}




\begin{tabular}{|c|c|c|c|c|c|c|c|}
\hline & & & & & & & $\begin{array}{l}\text { compared to low } \\
\text { trait anger } \\
\text { subjects }\end{array}$ \\
\hline $\begin{array}{l}\text { Hosseini } \\
(2011)^{67}\end{array}$ & Cross-sectional & $\begin{array}{l}\text { HTN \& NTN, } \\
\text { middle aged } \\
\text { adults }\end{array}$ & $100 \%$ & $\begin{array}{l}300 \\
33 \% \text { male }\end{array}$ & STAXI & $\begin{array}{l}\text { Manual } \\
\text { (standardization } \\
\text { level unclear) }\end{array}$ & $\begin{array}{l}\text { Trait anger more } \\
\text { common among } \\
\text { hypertensives }\end{array}$ \\
\hline $\begin{array}{l}\text { Markovitz } \\
(1991)^{69}\end{array}$ & $\begin{array}{l}\text { Longitudinal } \\
\text { follow-up ( } 3 \\
\text { years) }\end{array}$ & $\begin{array}{l}\text { NTN at baseline, } \\
\text { middle aged } \\
\text { adults }\end{array}$ & $7 \%$ & $\begin{array}{l}468 \\
0 \% \text { male }\end{array}$ & STAXI & $\begin{array}{l}\text { Standardized } \\
\text { manual } \\
\text { measurement }\end{array}$ & $\begin{array}{l}\text { Positive } \\
\text { association }\end{array}$ \\
\hline $\begin{array}{l}\text { Player } \\
(2007)^{74}\end{array}$ & $\begin{array}{l}\text { Longitudinal } \\
\text { follow-up (4-8 } \\
\text { years) }\end{array}$ & $\begin{array}{l}\text { Pre HTN at } \\
\text { baseline, middle } \\
\text { aged adults }\end{array}$ & $\begin{array}{l}\text { Reported as: } \\
20 \% \text { African } \\
\text { American, } \\
80 \% \text { not } \\
\text { identifying as } \\
\text { African } \\
\text { American }\end{array}$ & $\begin{array}{l}2,334 \\
48 \% \text { male }\end{array}$ & STAXI & $\begin{array}{l}\text { HTN established } \\
\text { by: 1) manual BP } \\
\text { measurement at } \\
\text { F/U, 2) prescription } \\
\text { of anti-HTN drugs } \\
\text { during F/U or 3) } \\
\text { High BP identified } \\
\text { by physician during } \\
\text { F/U }\end{array}$ & $\begin{array}{l}\text { Trait anger } \\
\text { positively } \\
\text { associated with } \\
\text { progression from } \\
\text { pre-hypertension } \\
\text { to hypertension } \\
\text { (males only) }\end{array}$ \\
\hline $\begin{array}{l}\text { Rohrmann } \\
(2011)^{75}\end{array}$ & $\begin{array}{l}\text { Interventional } \\
\text { design }\end{array}$ & $\begin{array}{l}\text { HTN status not } \\
\text { reported, young to } \\
\text { middle aged } \\
\text { adults }\end{array}$ & $\begin{array}{l}\text { NOT } \\
\text { REPORTED }\end{array}$ & $\begin{array}{l}82 \\
0 \% \text { male }\end{array}$ & STAXI & Automated office & $\begin{array}{l}\text { Positive } \\
\text { association }\end{array}$ \\
\hline $\begin{array}{l}\text { Crane } \\
(1981)^{66}\end{array}$ & Cross-sectional & $\begin{array}{l}\text { HTN \& NTN, } \\
\text { middle aged } \\
\text { adults }\end{array}$ & $0 \%$ & $\begin{array}{l}135 \\
100 \% \\
\text { male }\end{array}$ & STAXI & $\begin{array}{l}\text { N/A (HTN status } \\
\text { established by } \\
\text { referring physician) }\end{array}$ & $\begin{array}{l}\text { Higher trait } \\
\text { anger and T- } \\
\text { Ang/R reported } \\
\text { for HTN group }\end{array}$ \\
\hline $\begin{array}{l}\text { Johnson } \\
(1984)^{57}\end{array}$ & Cross-sectional & $\begin{array}{l}\text { NTN, high school } \\
\text { students }\end{array}$ & $42 \%$ & $\begin{array}{l}1060 \\
57 \% \text { male }\end{array}$ & STAXI & Automated office & No association \\
\hline
\end{tabular}

Acronyms: Blood pressure (BP), Blood pressure measurement (BPM), hypertension (HTN), white coat hypertension (WCH),

normotension (NTN), State-trait Anger Expression Inventory (STAXI), ambulatory blood pressure monitoring (ABPM), blood pressure (BP), systolic blood pressure (SBP), diastolic blood pressure (DBP), follow-up (F/U), trait anger reaction (T-Ang/R). 
Table 3. Studies examining anger control and blood pressure

\begin{tabular}{|l|l|l|l|l|l|l|l|}
\hline $\begin{array}{l}\text { Author } \\
\text { (year) }\end{array}$ & Study type & Population & $\begin{array}{l}\text { \% identify } \\
\text { as non- } \\
\text { Caucasian }\end{array}$ & $\begin{array}{l}\text { Sample size } \\
\% \text { male }\end{array}$ & $\begin{array}{l}\text { Anger } \\
\text { assessment } \\
\text { tool }\end{array}$ & BPM type & Findings \\
\hline $\begin{array}{l}\text { Igna } \\
(2009)^{24}\end{array}$ & $\begin{array}{l}\text { Cross- } \\
\text { sectional }\end{array}$ & $\begin{array}{l}\text { NTN, middle } \\
\text { aged adults }\end{array}$ & $\begin{array}{l}\text { NOT } \\
\text { REPORTED }\end{array}$ & 705 & $\begin{array}{l}\text { AX subscale } \\
\text { from STAXI }\end{array}$ & $\begin{array}{l}\text { Standardized } \\
\text { manual }\end{array}$ & Positive association \\
\hline $\begin{array}{l}\text { Ohira } \\
(2000)^{115}\end{array}$ & $\begin{array}{l}\text { Cross- } \\
\text { sectional }\end{array}$ & $\begin{array}{l}\text { All BP levels } \\
\text { but primarily } \\
\text { NTN, } \\
\text { Middle aged } \\
\text { males }\end{array}$ & $\begin{array}{l}100 \%(\text { all } \\
\text { Japanese) }\end{array}$ & $\begin{array}{l}790 \\
100 \%\end{array}$ & $\begin{array}{l}\text { AX subscale } \\
\text { from STAXI }\end{array}$ & $\begin{array}{l}\text { Standardized } \\
\text { manual }\end{array}$ & No association \\
\hline $\begin{array}{l}\text { Acronyms: Blood pressure measurement (BPM), Anger expression (AX), State trait anger expression inventory (STAXI), blood pressure } \\
(B P), \text { systolic blood pressure (SBP), diastolic blood pressure (DBP), normotensive (NTN) }\end{array}$
\end{tabular}


Table 4. Methodological inconsistencies in the field of anger expression and blood pressure research

- Differing self-report anger measurement tools

- Unreliable or inconsistent blood pressure measurement tools

- Narrow subject populations

- For example, primarily white middle aged females ${ }^{69}$ or female university students ${ }^{75}$

- Varying outcome measures

- Treating blood pressure as a continuous versus dichotomous variable. For example, blood pressure change vs. proportion of anger expression between hypertensive and normotensive subjects.

- Varying anger constructs and subcomponents investigated

- For example, studying only anger expressed in and anger expressed out and not other anger subcomponents ${ }^{62}$. Or even more narrow, studying only one component of anger expression, such as anger expressed in ${ }^{50}$

- Studying only diastolic blood pressure (an issue comparing older studies to more recent works) ${ }^{52,53}$

- Incorrect use of anger definitions and categorizations as defined by Spielberger ${ }^{118,225}$

- Example: Porter et al. in 1999 conducted a study titled "Anger expression and ambulatory blood pressure: A comparison of state and trait measures". However, using Spielberger's categorization, state and trait anger are subcomponents of anger 
experience, not anger expression. State and trait anger expression do not exist in Spielberger's framework for understanding anger. The authors did reliably measure Anger Expression using the appropriate subscale of the STAXI. However, they subsequently combined those findings with other measures of state and trait anger creating "trait and state measures of anger expression", again a concept that does not exist in Spielberger's framework for understanding anger. This cherry picking approach to terminology, definitions and classifications creates considerable confusion.

- Example: Suls et al. published at meta-analysis in 1995 titled "Relationship of Trait Anger to Resting Blood Pressure - a meta analysis". Several different types of anger and hostility self-report questionnaires were included. To review, Spielberger's categories are:

Anger Experience

- State Anger

- Trait Anger

Anger Expression

- Anger Expressed In

- Anger Expressed Out

- Anger Control In

- Anger Control Out 
In Suls et al.'s meta-analysis, studies that examined Spielberger's anger expression out subscale were categorized under "Anger Expression measures" and those that examined Spielberger's anger expression in subscale were categorized under "Anger Experience measures". These two categories (anger experience and anger expression) were then grouped together as "Trait Anger Measures" (see Table 6 in Suls et al.). Clearly, the authors did not use terminology or categorizations as defined by the original authors of those terms and categories. In fact, upon closer inspection, this meta-analysis did not look at trait anger in any capacity as defined by Spielberger. Yet, in the abstract it was stated that "anger experience was correlated with elevated BP" and even the study was titled "Relationship of Trait Anger to Resting Blood Pressure...". Upon breaking down the findings and re-framing them using Spielberger's categories, what these authors did find was that anger expressed in (what the authors defined as anger experience filed under the broad category of 'trait anger') was correlated to higher blood pressure. While one can appreciate the authors' attempts to add clarity to the literature, the misuse of definitions and categorizations makes interpreting findings extremely difficult. 
Table 5. Studies examining anger expression and blood pressure

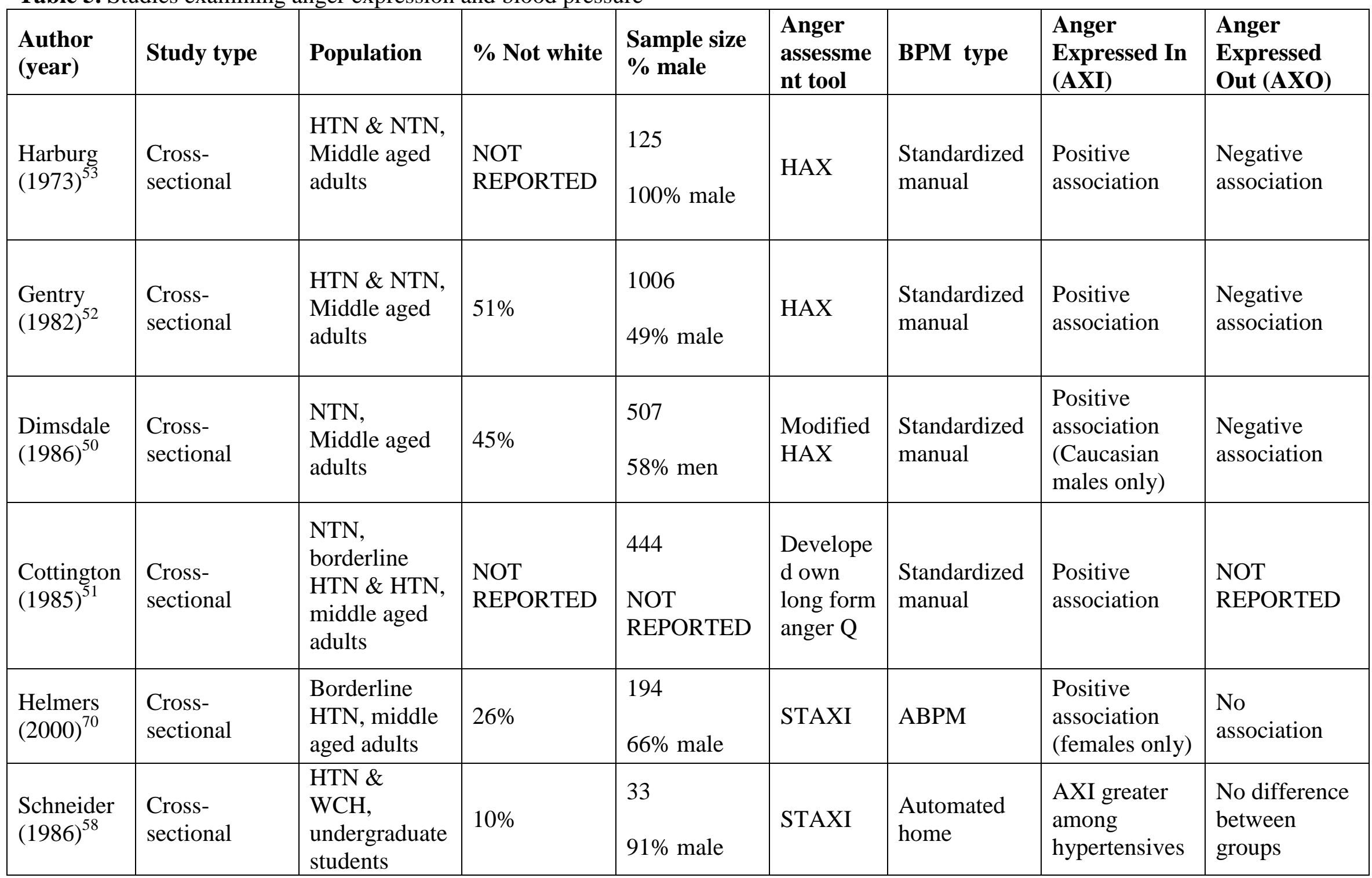




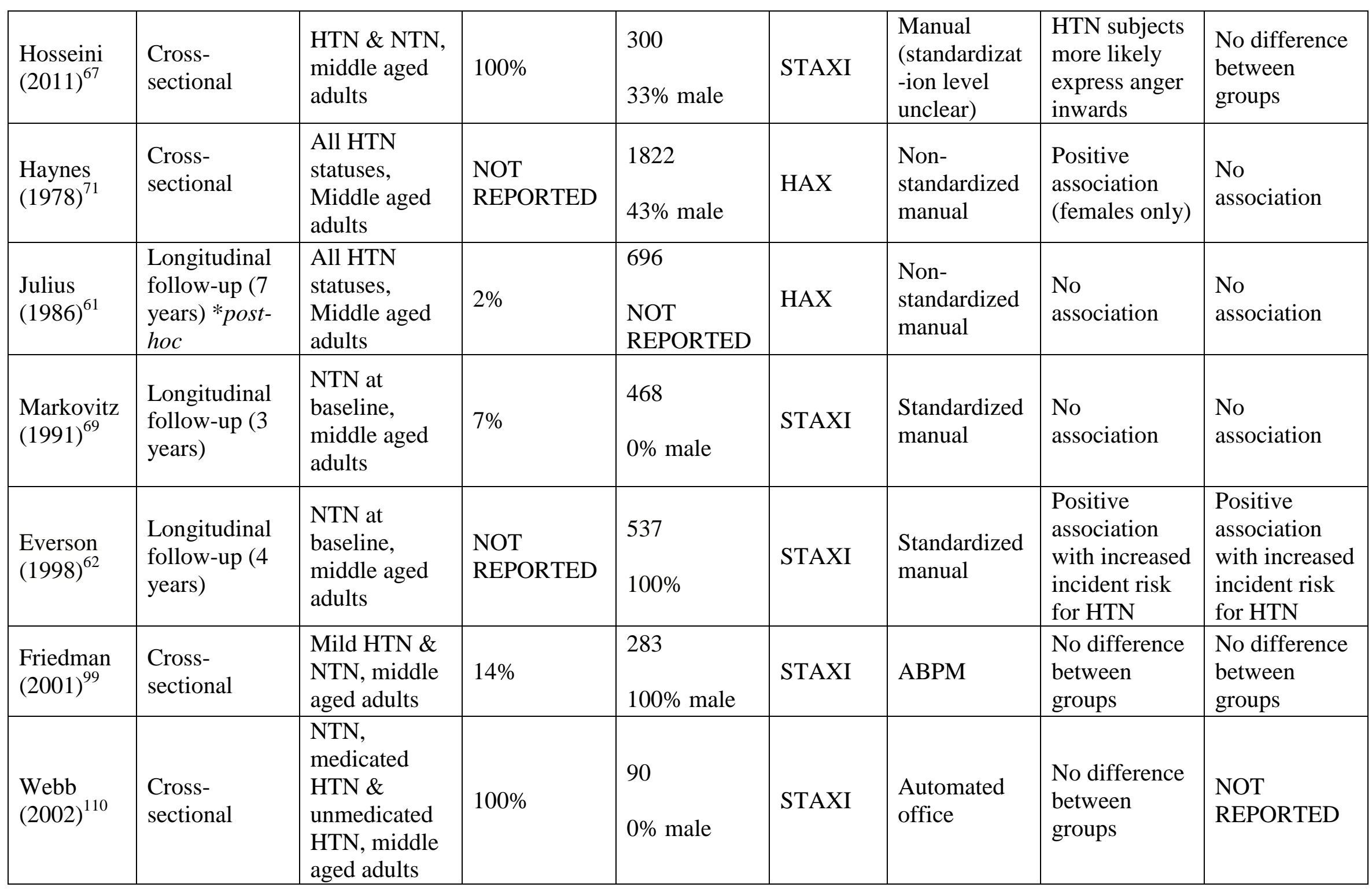




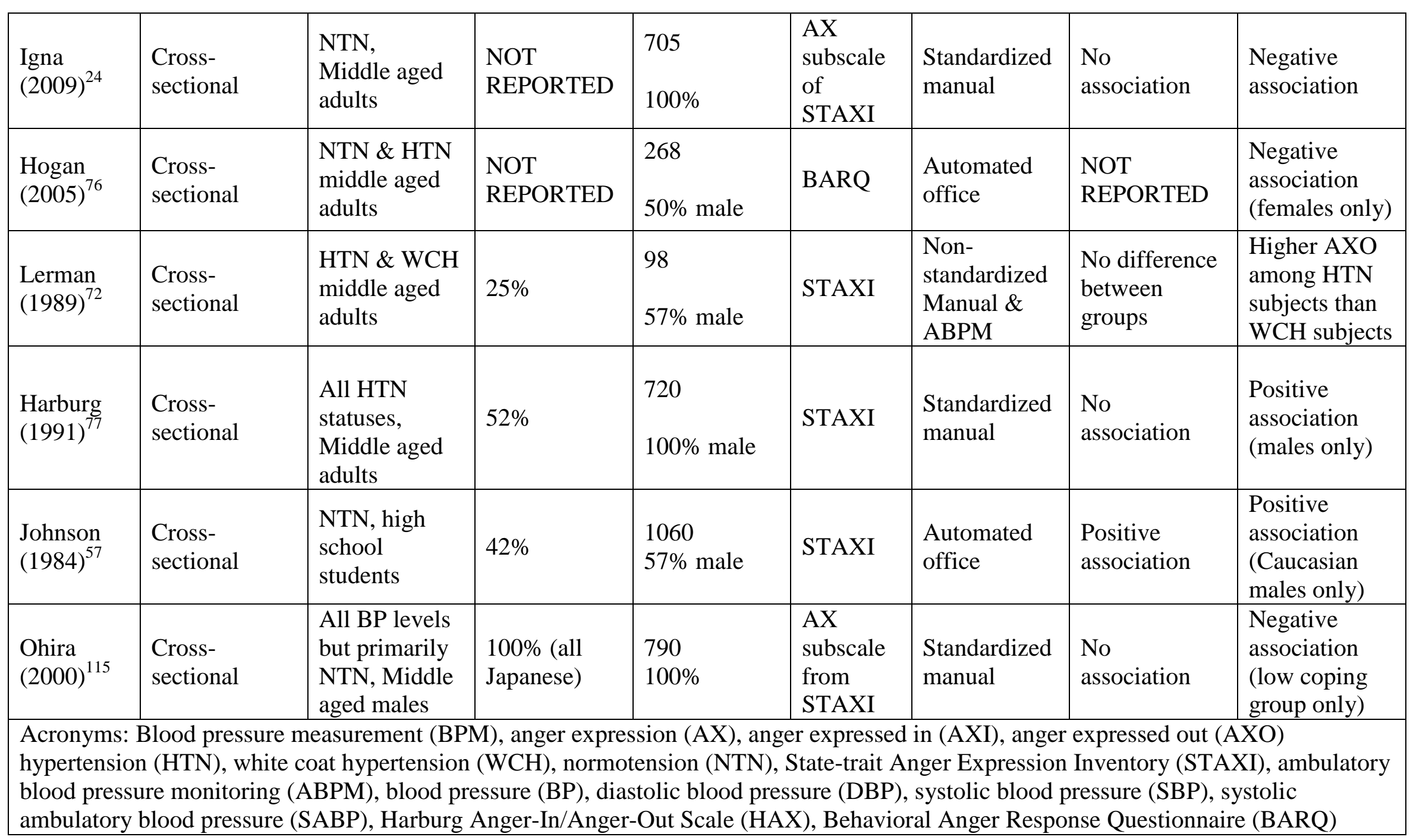


Table 6. Studies examining perceived stress and blood pressure

\begin{tabular}{|c|c|c|c|c|c|c|c|}
\hline $\begin{array}{l}\text { Author } \\
\text { (year) }\end{array}$ & Study type & Population & $\begin{array}{l}\% \text { Not } \\
\text { white }\end{array}$ & $\begin{array}{l}\text { Sample size } \\
\% \text { male }\end{array}$ & $\begin{array}{l}\text { Stress assessment } \\
\text { tool }\end{array}$ & BPM type & Finding \\
\hline $\begin{array}{l}\text { Huang } \\
(2011)^{83}\end{array}$ & $\begin{array}{l}\text { Cross- } \\
\text { sectional }\end{array}$ & $\begin{array}{l}\text { HTN, middle } \\
\text { aged adults }\end{array}$ & $100 \%$ & $\begin{array}{l}307 \\
52 \% \text { male }\end{array}$ & Cohen's PSS & ABPM & $\begin{array}{l}\text { Positive } \\
\text { association }\end{array}$ \\
\hline $\begin{array}{l}\text { Wright } \\
(2011)^{84}\end{array}$ & $\begin{array}{l}\text { Longitudinal } \\
\text { follow-up ( } 6 \\
\text { years) }\end{array}$ & $\begin{array}{l}\text { NTN, middle } \\
\text { aged adults }\end{array}$ & $\begin{array}{l}\text { NOT } \\
\text { REPORTED }\end{array}$ & $\begin{array}{l}170 \\
100 \% \text { male }\end{array}$ & Cohen's PSS & $\begin{array}{l}\text { Standardized } \\
\text { manual }\end{array}$ & $\begin{array}{l}\text { Positive } \\
\text { association }\end{array}$ \\
\hline $\begin{array}{l}\text { Logan } \\
(2012)^{134}\end{array}$ & $\begin{array}{l}\text { Cross- } \\
\text { sectional }\end{array}$ & $\begin{array}{l}\text { NTN, middle } \\
\text { aged adults }\end{array}$ & $100 \%$ & $\begin{array}{l}102 \\
40 \% \text { male }\end{array}$ & Cohen's PSS & $\begin{array}{l}\text { Automated } \\
\text { office }\end{array}$ & No association \\
\hline $\begin{array}{l}\text { Rod } \\
(2009)^{85}\end{array}$ & $\begin{array}{l}\text { Longitudinal } \\
\text { follow-up } \\
\text { (10 years) }\end{array}$ & $\begin{array}{l}\text { HTN status not } \\
\text { reported, } \\
\text { middle aged } \\
\text { adults }\end{array}$ & $\begin{array}{l}\text { NOT } \\
\text { REPORTED }\end{array}$ & $\begin{array}{l}7066 \\
42 \% \text { male }\end{array}$ & $\begin{array}{l}\text { Non validated PSS } \\
2 \text { item Q that } \\
\text { addressed level of PS } \\
\& \text { frequency of PS }\end{array}$ & $\begin{array}{l}\text { N/A (authors } \\
\text { measured } \\
\text { change in } \\
\text { HTN status) }\end{array}$ & $\begin{array}{l}\text { High PS } \\
\text { association with } \\
\text { HTN at F/U }\end{array}$ \\
\hline
\end{tabular}




\begin{tabular}{|c|c|c|c|c|c|c|c|}
\hline $\begin{array}{l}\text { Pollard } \\
(2007)^{78}\end{array}$ & $\begin{array}{l}\text { Cross- } \\
\text { sectional }\end{array}$ & $\begin{array}{l}\text { NTN, middle } \\
\text { aged adults }\end{array}$ & $\begin{array}{l}\text { NOT } \\
\text { REPORTED }\end{array}$ & $\begin{array}{l}33 \\
0 \% \text { male }\end{array}$ & $\begin{array}{l}\text { Non validated PSS } \\
1 \text { item } \mathrm{Q} \text { on PS, } 1= \\
\text { not at all stressed to } 7 \\
=\text { very stressed. }\end{array}$ & $\begin{array}{l}\text { Automated } \\
\text { home }\end{array}$ & $\begin{array}{l}\text { Positive } \\
\text { association }\end{array}$ \\
\hline $\begin{array}{l}\text { Kario } \\
(2002)^{79}\end{array}$ & $\begin{array}{l}\text { Cross- } \\
\text { sectional }\end{array}$ & $\begin{array}{l}\text { NTN, middle } \\
\text { aged adults, PS } \\
\text { at work vs. PS } \\
\text { at home }\end{array}$ & $100 \%$ & $\begin{array}{l}135 \\
0 \% \text { male }\end{array}$ & $\begin{array}{l}\text { Non validated PSS } \\
1 \text { item } Q \text { on PS, } 0= \\
\text { low stress to } 10=\text { high } \\
\text { stress }\end{array}$ & ABPM & $\begin{array}{l}\text { Higher work BP \& } \\
\text { sleep SBP among } \\
\text { subjects reporting } \\
\text { high PS at work } \\
\text { vs. high PS at } \\
\text { home }\end{array}$ \\
\hline $\begin{array}{l}\text { James } \\
(2001)^{80}\end{array}$ & $\begin{array}{l}\text { Cross- } \\
\text { sectional }\end{array}$ & $\begin{array}{l}\text { NTN, young - } \\
\text { middle aged } \\
\text { adults, PS at } \\
\text { work vs. PS at } \\
\text { home }\end{array}$ & $61.5 \%$ & $\begin{array}{l}91 \\
0 \% \text { male }\end{array}$ & $\begin{array}{l}\text { Non validated PSS } \\
1 \text { item } Q \text { on PS, } 0= \\
\text { low stress to } 10=\text { high } \\
\text { stress }\end{array}$ & ABPM & $\begin{array}{l}\text { Work-stressed } \\
\text { subjects had } \\
\text { higher BP than } \\
\text { home-stressed } \\
\text { subjects }\end{array}$ \\
\hline $\begin{array}{l}\text { Langewitz } \\
(1987)^{82}\end{array}$ & $\begin{array}{l}\text { Cross- } \\
\text { sectional }\end{array}$ & $\begin{array}{l}\text { NTN, NOT } \\
\text { REPORTED }\end{array}$ & $\begin{array}{l}\text { NOT } \\
\text { REPORTED }\end{array}$ & $\begin{array}{l}34 \\
\text { NOT } \\
\text { REPORTED }\end{array}$ & $\begin{array}{l}\text { Non validated PSS } \\
1 \text { item Q on PS, } 1= \\
\text { completely relaxed to } \\
5=\text { extremely stressed }\end{array}$ & ABPM & $\begin{array}{l}\text { Positive } \\
\text { association }\end{array}$ \\
\hline
\end{tabular}




\begin{tabular}{|c|c|c|c|c|c|c|c|}
\hline $\begin{array}{l}\text { Moya- } \\
\text { Albiol } \\
(2010)^{140}\end{array}$ & $\begin{array}{l}\text { Cross- } \\
\text { sectional }\end{array}$ & $\begin{array}{l}\text { NTN, Middle } \\
\text { aged adults }\end{array}$ & $\begin{array}{l}\text { NOT } \\
\text { REPORTED }\end{array}$ & $\begin{array}{l}64 \\
25 \% \text { male }\end{array}$ & $\begin{array}{l}\text { Non validated PSS } \\
\text { Multiple item Q on } \\
\text { PS, more elaborate, PS } \\
\text { evaluated using Likert } \\
\text { four point scale }\end{array}$ & $\begin{array}{l}\text { Automated } \\
\text { office }\end{array}$ & No association \\
\hline $\begin{array}{l}\text { Strayhorn } \\
(1980)^{143}\end{array}$ & $\begin{array}{l}\text { Cross- } \\
\text { sectional }\end{array}$ & $\begin{array}{l}\text { NTN, } \\
\text { university } \\
\text { students }\end{array}$ & $18 \%$ & $\begin{array}{l}104 \\
66 \% \text { male }\end{array}$ & $\begin{array}{l}\text { Non validated PSS } \\
\text { Multiple item Q on } \\
\text { PS, more elaborate, } \\
\text { derived from literature } \\
\text { on stress } \\
\text { in medical school }\end{array}$ & $\begin{array}{l}\text { Non- } \\
\text { standardized } \\
\text { manual }\end{array}$ & $\begin{array}{l}\text { No differences in } \\
\text { BP between low } \\
\text { and high stress } \\
\text { groups }\end{array}$ \\
\hline $\begin{array}{l}\text { Suter } \\
(1997)^{142}\end{array}$ & $\begin{array}{l}\text { Cross- } \\
\text { sectional }\end{array}$ & $\begin{array}{l}\text { All HTN } \\
\text { statuses, } \\
\text { Middle aged } \\
\text { adults }\end{array}$ & $0 \%$ & $\begin{array}{l}1601 \\
69 \% \text { male }\end{array}$ & $\begin{array}{l}\text { Non validated PSS } \\
1 \text { item Q on PS, } \\
\text { measured using a } \\
\text { single visual analog } \\
\text { scale }\end{array}$ & $\begin{array}{l}\text { Automated } \\
\text { office }\end{array}$ & $\begin{array}{l}\text { Negative } \\
\text { association }\end{array}$ \\
\hline
\end{tabular}


Table 7. Study inclusion and exclusion criteria. Adapted from the HARMONY protocol manuscript $^{1}$

\section{Inclusion criteria:}

1. Age 20 to 75 years.

2. Stage- 1 hypertensive as determined by ABPM at baseline (daytime $\geq 135 / 85 \mathrm{mmHg}$ or 24-hour $\mathrm{ABPM} \geq 130 / 80 \mathrm{mmHg}$ ).

3. Willing and able to participate in the MBSR program.

4. Willing to be followed for safety BP checks if necessary.

5. Willing to accept a possible waiting period for MBSR.

6. Written informed consent.

7. Able to participate in mindfulness meditation program.

\section{Exclusion criteria:}

1. Use of antihypertensive medication within 6 months of the screening ABPM.

2. Screening office $B P>180 / 100 \mathrm{mmHg}$ and $A B P M \geq 160 / 100 \mathrm{mmHg}$.

3. Diabetes.

4. Secondary hypertension.

5. Renal disease (glomerular filtration rate $<60 \mathrm{ml} / \mathrm{min}$ or overt nephropathy).

6. History of heart attack.

7. Stroke or transient ischemic attack.

8. Re-vascularization procedure.

9. Active malignant disease (except non-melanoma skin cancer).

10. Epileptic seizure 6 months before the screening visit.

11. Congestive heart failure.

12. Severe liver disease.

13. Pregnancy or lactation period.

14. Participation in a clinical trail or receipt of investigational compound or treatment in the 3 months prior to the initial screening visit.

15. Planned elective surgery during the study period except for cataract surgery.

16. Inability or unwillingness to perform ABPMs.

$\mathrm{ABPM}=$ ambulatory blood pressure monitoring, $\mathrm{MBSR}=$ mindfulness-based stress reduction, $\mathrm{BP}=$ blood pressure. 
Table 8. Brief overview of the STAXI-2 scales and subscales. Adapted from STAXI-2 manual ${ }^{2}$

\begin{tabular}{|c|c|c|c|}
\hline $\begin{array}{l}\text { STAXI-2 } \\
\text { scale/subscale }\end{array}$ & $\begin{array}{c}\text { Number of } \\
\text { questions }\end{array}$ & $\begin{array}{c}\text { Scale/subscale } \\
\text { range }\end{array}$ & Description of scale/subscale \\
\hline State Anger (S-Ang) & 15 & $15-60$ & $\begin{array}{l}\text { Measures the intensity of angry } \\
\text { feelings and the extent to which a } \\
\text { person feels like expressing anger at a } \\
\text { particular time }\end{array}$ \\
\hline $\begin{array}{l}\text { Feeling Angry } \\
(\mathrm{S}-\mathrm{Ang} / \mathrm{F})\end{array}$ & 5 & $5-20$ & $\begin{array}{l}\text { Measures the intensity of the angry } \\
\text { feelings the person is currently } \\
\text { experiencing }\end{array}$ \\
\hline $\begin{array}{l}\text { Feel Like } \\
\text { Expressing } \\
\text { Anger Verbally } \\
(\mathrm{S}-\text { Ang/F) }\end{array}$ & 5 & $5-20$ & $\begin{array}{l}\text { Measures the intensity of current } \\
\text { feelings related to the verbal } \\
\text { expression of anger }\end{array}$ \\
\hline $\begin{array}{l}\text { Feel Like } \\
\text { Expressing } \\
\text { Anger } \\
\text { Physically (S- } \\
\text { Ang/F) }\end{array}$ & 5 & $5-20$ & $\begin{array}{l}\text { Measures the intensity of current } \\
\text { feelings related to the physical } \\
\text { expression of anger }\end{array}$ \\
\hline Trait Anger (T-Ang) & 10 & $10-40$ & $\begin{array}{l}\text { Measures how often angry feelings are } \\
\text { experienced over time }\end{array}$ \\
\hline $\begin{array}{l}\text { Angry } \\
\text { Temperament } \\
(\mathrm{T}-\text { Ang/T) } \\
\end{array}$ & 4 & $4-16$ & $\begin{array}{l}\text { Measures the disposition to experience } \\
\text { angry without specific provocation }\end{array}$ \\
\hline $\begin{array}{l}\text { Angry Reaction } \\
(\mathrm{T}-\text { Ang/R) }\end{array}$ & 4 & $4-16$ & $\begin{array}{l}\text { Measures the frequency that angry } \\
\text { feelings are experienced in situations } \\
\text { that involve frustration and/or negative } \\
\text { evaluations. }\end{array}$ \\
\hline $\begin{array}{l}\text { Anger Expression In } \\
\text { (AXI) }\end{array}$ & 8 & $8-32$ & $\begin{array}{l}\text { Measures how often angry feelings are } \\
\text { experienced but not expressed } \\
\text { (suppressed) }\end{array}$ \\
\hline $\begin{array}{l}\text { Anger Expression } \\
\text { Out (AXO) }\end{array}$ & 8 & $8-32$ & $\begin{array}{l}\text { Measures how often angry feelings are } \\
\text { expressed in verbally or physically } \\
\text { aggressive behaviour }\end{array}$ \\
\hline $\begin{array}{l}\text { Anger Control In } \\
(\mathrm{ACI})\end{array}$ & 8 & $8-32$ & $\begin{array}{l}\text { Measures how often a person attempts } \\
\text { to control angry feelings by calming } \\
\text { down or cooling off }\end{array}$ \\
\hline $\begin{array}{l}\text { Anger Control Out } \\
(\mathrm{ACO})\end{array}$ & 8 & $8-32$ & $\begin{array}{l}\text { Measures how often a person controls } \\
\text { the outwards expression of angry } \\
\text { feelings }\end{array}$ \\
\hline $\begin{array}{l}\text { Anger Expression } \\
\text { Index }(\mathrm{AXX})\end{array}$ & 32 & $0-96$ & $\begin{array}{l}\text { Provides a general index of anger } \\
\text { expression based on responses to the } \\
\text { AXI, AXO, ACI and ACO items. }\end{array}$ \\
\hline
\end{tabular}


Table 9. Baseline demographic data

\begin{tabular}{|lc|}
\hline Characteristics & Result \\
\hline Sex (\% male) & $37 \%$ \\
Age (years) & $56(11.4)$ \\
Waist circ. (cm) & $90(11.5)$ \\
BMI (kg/m^2) & $28(4.8)$ \\
Office SBP & $136(13.0)$ \\
Office DBP & $86(7.2)$ \\
24 HR SBP & $135(7.5)$ \\
24 HR DBP & $81(5.6)$ \\
Daytime SBP & $141(7.5)$ \\
Daytime DBP & $86(6.0)$ \\
Nighttime SBP & $122(10.3)$ \\
Nighttime DBP & $71(7.0)$ \\
Family History of Hypertension (\%) & $62 \%$ \\
Family history of premature coronary heart & \\
disease & $28 \%$ \\
$\geq$ Post-Secondary Education (\% yes) & $86 \%$ \\
Working (\%) & $80 \%$ \\
Drinks $>$ 10/week & $20 \%$ \\
Use relaxation techniques on a regular basis & $17 \%$ \\
Vigorous exercise & $64 \%$ \\
Moderate exercise & $91 \%$ \\
Self-identify as Caucasian & minutes. \\
\hline Data is reported in mean (SD) for continuous variables. SBP systolic \\
blood pressure, DBP = diastolic blood pressure. All BP \\
mmHg. Vigorous exercise = participation in any weekly vigorous \\
aerobic exercise lasting 20 $\geq$ minutes. Moderate exercise $=$ \\
participation in any weekly moderate exercise lasting \\
\hline
\end{tabular}


Table 10. Baseline psychosocial data

\begin{tabular}{|lcc|}
\hline Psychometric Scale & Score & \%ile Normal Adults* \\
\hline Trait Anger & $17(5)$ & $45^{\text {th }}$ \\
$\quad$ Angry Temperament & $7(3)$ & $65^{\text {th }}$ \\
Angry Reaction & $8(3)$ & $35^{\text {th }}$ \\
Anger Expression In & $17(4)$ & $60^{\text {th }}$ \\
Anger Expression Out & $14(3)$ & $40^{\text {th }}$ \\
Anger Control In & $22(6)$ & $45^{\text {th }}$ \\
Anger Control Out & $23(5)$ & $45^{\text {th }}$ \\
Anger Expression Index (AX & $34(14)$ & $50^{\text {th }}$ \\
index) & $24(8)$ & \\
\hline Perceived Stress Scale & & \\
Data is reported in mean (SD).Percentile ranks were obtained from the State-Trait Anger \\
Expression Inventory-2 Professional Manual. No such percentile ranking exists for the Perceived \\
Stress Scale.
\end{tabular}

Table 11. MBSR class attendance and homework completion

\begin{tabular}{|l|l|}
\hline $\begin{array}{l}\text { Average No. MBSR sessions attended out of } 8 \\
\text { (classes) }\end{array}$ & $6(1)$ \\
\hline $\begin{array}{l}\text { Average \# minutes of MBSR homework/day } \\
\text { (minutes) }\end{array}$ & $32(15.2)$ \\
\hline Data is reported in mean $(S D)$. & \\
\hline
\end{tabular}


Table 12. Correlations between baseline trait anger, trait anger temperament, trait anger reaction, anger expression inwards, anger expression outwards, perceived stress and baseline 24-hour blood pressure

\begin{tabular}{|c|c|c|c|c|c|c|}
\hline \multirow[b]{2}{*}{ Anger Measure } & \multicolumn{2}{|c|}{ All Subjects } & \multicolumn{2}{|l|}{ Female } & \multicolumn{2}{|l|}{ Male } \\
\hline & Systolic & Diastolic & Systolic & Diastolic & Systolic & Diastolic \\
\hline T-Ang & -0.018 & -0.043 & -0.040 & 0.017 & 0.040 & -0.139 \\
\hline T-Ang/T & -0.027 & -0.052 & -0.087 & -0.030 & 0.078 & -0.139 \\
\hline T-Ang/R & -0.064 & -0.002 & -0.030 & 0.065 & -0.096 & -0.118 \\
\hline AXI & -0.042 & 0.046 & 0.098 & -0.002 & -0.223 & 0.183 \\
\hline $\mathrm{AXO}$ & -0.120 & -0.104 & -0.079 & -0.099 & -0.163 & -0.117 \\
\hline PS & 0.081 & 0.098 & 0.203 & 0.155 & -0.121 & -0.035 \\
\hline
\end{tabular}

Table 13. Correlations between baseline trait anger, trait anger temperament, trait anger reaction, anger expression inwards, anger expression outwards, perceived stress and baseline daytime blood pressure

\begin{tabular}{|c|c|c|c|c|c|c|}
\hline \multirow[b]{2}{*}{$\begin{array}{l}\text { Anger } \\
\text { Measure }\end{array}$} & \multicolumn{2}{|c|}{ All Subjects } & \multicolumn{2}{|l|}{ Female } & \multicolumn{2}{|l|}{ Male } \\
\hline & Systolic & Diastolic & Systolic & Diastolic & Systolic & Diastolic \\
\hline T-Ang & -0.050 & -0.048 & -0.093 & 0.032 & 0.015 & -0.172 \\
\hline T-Ang/T & -0.082 & -0.051 & -0.142 & 0.002 & -0.008 & -0.148 \\
\hline T-Ang/R & -0.084 & 0.002 & -0.040 & 0.107 & -0.133 & -0.171 \\
\hline AXI & -0.137 & -0.032 & 0.004 & -0.076 & $-0.340 * *$ & 0.072 \\
\hline $\mathrm{AXO}$ & -0.160 & -0.115 & -0.177 & -0.136 & -0.129 & -0.081 \\
\hline PS & 0.022 & 0.065 & 0.143 & 0.142 & -0.174 & -0.106 \\
\hline \multicolumn{7}{|c|}{$\begin{array}{l}\text { Note. }{ }^{*} \mathrm{p}<0.10,{ }^{*} \mathrm{p}<0.05 . \mathrm{T}-\mathrm{Ang}=\text { Trait Anger, } \mathrm{T}-\mathrm{Ang} / \mathrm{T}=\text { Trait Anger Temperament, T-Ang/R } \\
=\text { Trait Anger Reaction, AXI = Anger Expression Inwards, AXO = Anger Expression Outwards, } \\
\text { PS = Perceived Stress. }\end{array}$} \\
\hline
\end{tabular}


Table 14. Correlations between baseline trait anger, trait anger temperament, trait anger reaction, anger expression inwards, anger expression outwards, perceived stress and baseline nighttime blood pressure

\begin{tabular}{|c|c|c|c|c|c|c|}
\hline \multirow[b]{2}{*}{ Anger Measure } & \multicolumn{2}{|c|}{ All Subjects } & \multicolumn{2}{|l|}{ Female } & \multicolumn{2}{|l|}{ Male } \\
\hline & Systolic & Diastolic & Systolic & Diastolic & Systolic & Diastolic \\
\hline T-Ang & 0.008 & -0.047 & 0.014 & -0.044 & 0.077 & -0.020 \\
\hline T-Ang/T & 0.037 & -0.021 & -0.016 & -0.037 & 0.203 & -0.023 \\
\hline T-Ang/R & -0.066 & -0.041 & -0.055 & -0.051 & -0.043 & -0.007 \\
\hline AXI & 0.059 & 0.116 & 0.180 & 0.126 & 0.006 & 0.188 \\
\hline $\mathrm{AXO}$ & 0.003 & -0.019 & 0.092 & 0.015 & -0.084 & -0.043 \\
\hline PS & 0.104 & 0.087 & $0.224 *$ & 0.122 & -0.058 & 0.060 \\
\hline \multicolumn{7}{|c|}{$\begin{array}{l}\text { Note. }{ }^{*} \mathrm{p}<0.10,{ }^{*} \mathrm{p}<0.05 . \mathrm{T}-\mathrm{Ang}=\text { Trait Anger, } \mathrm{T}-\mathrm{Ang} / \mathrm{T}=\text { Trait Anger Temperament, T-Ang/R } \\
=\text { Trait Anger Reaction, AXI = Anger Expression Inwards, AXO = Anger Expression Outwards, } \\
\text { PS = Perceived Stress. }\end{array}$} \\
\hline
\end{tabular}


Table 15. Correlations between baseline anger control inwards, anger control outwards, anger expression index and baseline 24-hour blood pressure

\begin{tabular}{|lllllll|}
\hline & \multicolumn{2}{l}{ All Subjects } & Female & \multicolumn{3}{c|}{ Male } \\
\cline { 2 - 6 } Anger Measure & Systolic & Diastolic & Systolic & Diastolic & Systolic & Diastolic \\
\hline ACI & 0.158 & 0.010 & 0.117 & 0.067 & 0.180 & -0.151 \\
ACO & 0.048 & 0.092 & -0.019 & 0.086 & 0.136 & 0.094 \\
AXX & -0.120 & -0.046 & -0.025 & -0.078 & -0.248 & 0.059 \\
& & & & & & \\
Note. $*$ p $<0.10, * * p<0.05$. ACI $=$ Anger Control Inwards, ACO = Anger Control Outwards, \\
AXX = Anger Expression Index.
\end{tabular}

Table 16. Correlations between baseline anger control inwards, anger control outwards, anger expression index and baseline daytime blood pressure

\begin{tabular}{|c|c|c|c|c|c|c|}
\hline \multirow[b]{2}{*}{ Anger Measure } & \multicolumn{2}{|c|}{ All Subjects } & \multicolumn{2}{|l|}{ Female } & \multicolumn{2}{|l|}{ Male } \\
\hline & Systolic & Diastolic & Systolic & Diastolic & Systolic & Diastolic \\
\hline ACI & $0.207 * *$ & 0.022 & 0.154 & 0.030 & $0.282 *$ & -0.015 \\
\hline $\mathrm{ACO}$ & 0.103 & 0.120 & 0.061 & 0.104 & 0.161 & 0.152 \\
\hline $\mathrm{AXX}$ & $-0.198 *$ & -0.088 & -0.117 & -0.100 & $-0.330 * *$ & -0.047 \\
\hline
\end{tabular}

Table 17. Correlations between baseline anger control inwards, anger control outwards, anger expression index and baseline nighttime blood pressure

\begin{tabular}{|c|c|c|c|c|c|c|}
\hline \multirow[b]{2}{*}{ Anger Measure } & \multicolumn{2}{|c|}{ All Subjects } & \multicolumn{2}{|l|}{ Female } & \multicolumn{2}{|l|}{ Male } \\
\hline & Systolic & Diastolic & Systolic & Diastolic & Systolic & Diastolic \\
\hline $\mathrm{ACI}$ & 0.088 & 0.030 & 0.049 & 0.128 & 0.007 & -0.235 \\
\hline $\mathrm{ACO}$ & -0.005 & 0.042 & -0.093 & 0.062 & 0.052 & -0.061 \\
\hline $\mathrm{AXX}$ & -0.014 & 0.005 & 0.087 & -0.032 & -0.043 & 0.173 \\
\hline
\end{tabular}


Table 18. Changes in trait anger, trait anger temperament, trait anger reaction, anger expressed inwards, anger expressed outwards, perceived stress, anger control inwards, anger control outwards and the anger expression index from pre to post intervention

\begin{tabular}{|c|c|c|c|c|}
\hline & Pre & Post & $\begin{array}{l}\text { Average } \\
\text { Change }\end{array}$ & Change p-value \\
\hline \multicolumn{5}{|l|}{ Trait anger } \\
\hline All Subjects & $17.03(5.26)$ & $15.56(4.00)$ & $-1.47(3.30)$ & $0.0003 *$ \\
\hline Female & $16.82(4.83)$ & $15.55(3.80)$ & $-1.27(3.18)$ & $0.0076 *$ \\
\hline Male & $17.42(6.05)$ & $15.58(4.44)$ & $-1.85(3.55)$ & $0.0137 *$ \\
\hline \multicolumn{5}{|c|}{ Trait anger temperament } \\
\hline All Subjects & $6.53(2.67)$ & $5.73(1.91)$ & $-0.80(1.60)$ & $0.0025 *$ \\
\hline Female & $6.37(2.44)$ & $5.57(1.77)$ & $-0.80(1.62)$ & $0.0241 *$ \\
\hline Male & $6.85(3.08)$ & $6.04(2.14)$ & $-0.81(1.60)$ & 0.0768 \\
\hline \multicolumn{5}{|c|}{ Trait anger reaction } \\
\hline All Subjects & $7.71(2.57)$ & $7.22(2.28)$ & $-0.50(1.79)$ & $0.0190 *$ \\
\hline Female & $7.73(2.41)$ & $7.42(2.11)$ & $-0.33(1.75)$ & 0.1944 \\
\hline Male & $7.65(2.90)$ & $6.85(2.57)$ & $-0.81(1.86)$ & $0.0357 *$ \\
\hline \multicolumn{5}{|c|}{ Anger expression inwards } \\
\hline All Subjects & $16.73(4.43)$ & $14.84(3.77)$ & $-1.89(4.20)$ & $0.0002 *$ \\
\hline Female & $16.10(4.40)$ & $14.45(3.52)$ & $-1.65(3.55)$ & $0.0021 *$ \\
\hline Male & $17.92(4.34)$ & $15.58(4.18)$ & $-2.35(5.26)$ & $0.0318 *$ \\
\hline \multicolumn{5}{|c|}{ Anger expression outwards } \\
\hline All Subjects & $13.97(3.18)$ & $13.15(2.60)$ & $-0.83(2.93)$ & $0.0171 *$ \\
\hline Female & $13.82(3.15)$ & $12.80(2.50)$ & $-1.02(2.87)$ & $0.0163 *$ \\
\hline Male & $14.27(3.28)$ & $13.81(2.71)$ & $-0.46(3.08)$ & 0.4513 \\
\hline \multicolumn{5}{|c|}{ Perceived Stress } \\
\hline All Subjects & $24.04(8.18)$ & $20.77(6.34)$ & $-3.21(7.24)$ & $0.0001 *$ \\
\hline Female & $23.60(8.51)$ & $20.50(6.58)$ & $-2.98(7.09)$ & $0.0038 *$ \\
\hline Male & $24.77(7.66)$ & $21.24(5.98)$ & $-3.62(7.60)$ & $0.0160 *$ \\
\hline \multicolumn{5}{|c|}{ Anger control inwards } \\
\hline All Subjects & $21.67(5.82)$ & $24.76(4.68)$ & $3.09(4.45)$ & $<.0001 *$ \\
\hline Female & $22.47(5.83)$ & $25.45(4.87)$ & $2.98(4.30)$ & $<.0001 *$ \\
\hline Male & $20.15(5.60)$ & $23.46(4.09)$ & $3.31(4.81)$ & $0.0017 *$ \\
\hline \multicolumn{5}{|c|}{ Anger control outwards } \\
\hline All Subjects & $23.31(5.42)$ & $24.87(5.01)$ & $1.56(3.59)$ & $0.0003 *$ \\
\hline Female & $23.76(5.07)$ & $25.59(4.81)$ & $1.84(3.60)$ & $0.0008 *$ \\
\hline Male & $22.46(4.83)$ & $25.30(5.18)$ & $1.04(3.57)$ & 0.1507 \\
\hline \multicolumn{5}{|c|}{ Anger expression index } \\
\hline All Subjects & $33.73(14.94)$ & $26.36(12.20)$ & $-7.37(10.66)$ & $<.0001 *$ \\
\hline Female & $31.69(15.54)$ & $24.20(12.21)$ & $-7.49(9.86)$ & $<.0001 *$ \\
\hline Male & $37.58(13.16)$ & $30.42(11.31)$ & $-7.15(12.24)$ & $0.0063 *$ \\
\hline
\end{tabular}


Table 19. Changes in the proportion of those scoring greater than or equal to the $75^{\text {th }}$ percentile in trait anger, trait anger temperament, trait anger reaction, anger expressed inwards, anger expressed outwards, anger control inwards, anger control outwards and anger expression from pre to post intervention

\begin{tabular}{|c|c|c|c|}
\hline & $\begin{array}{l}\text { Proportion } \geq 75^{\text {th }} \\
\text { percentile }(\mathbf{P R E})\end{array}$ & $\begin{array}{l}\text { Proportion } \geq 75^{\text {th }} \text { percentile } \\
(\text { POST) }\end{array}$ & p-value \\
\hline \multicolumn{4}{|l|}{ Trait Anger } \\
\hline All Subjects & $24 \%$ & $13 \%$ & $0.0215^{*}$ \\
\hline Female & $22 \%$ & $12 \%$ & 0.1250 \\
\hline Male & $31 \%$ & $19 \%$ & 0.2500 \\
\hline \multicolumn{4}{|c|}{ Trait Anger Temperament } \\
\hline All Subjects & $32 \%$ & $23 \%$ & $0.0391 *$ \\
\hline Female & $37 \%$ & $20 \%$ & $0.0078 *$ \\
\hline Male & $35 \%$ & $31 \%$ & 1.0000 \\
\hline \multicolumn{4}{|c|}{ Trait Anger Reaction } \\
\hline All Subjects & $16 \%$ & $7 \%$ & $0.0391 *$ \\
\hline Female & $15 \%$ & $6 \%$ & 0.2188 \\
\hline Male & $19 \%$ & $15 \%$ & 1.0000 \\
\hline \multicolumn{4}{|c|}{ Anger Expression Inwards } \\
\hline All Subjects & $32 \%$ & $12 \%$ & $0.0015^{*}$ \\
\hline Female & $41 \%$ & $12 \%$ & $0.0013 *$ \\
\hline Male & $54 \%$ & $27 \%$ & 0.0654 \\
\hline \multicolumn{4}{|c|}{ Anger Expression Outwards } \\
\hline All Subjects & $17 \%$ & $8 \%$ & 0.0654 \\
\hline Female & $24 \%$ & $16 \%$ & 0.3438 \\
\hline Male & $23 \%$ & $15 \%$ & 0.6250 \\
\hline \multicolumn{4}{|c|}{ Anger Control Inwards } \\
\hline All Subjects & $19 \%$ & $35 \%$ & $0.0042 *$ \\
\hline Female & $18 \%$ & $27 \%$ & $0.0225^{*}$ \\
\hline Male & $12 \%$ & $19 \%$ & 0.6250 \\
\hline \multicolumn{4}{|c|}{ Anger Control Outwards } \\
\hline All Subjects & $27 \%$ & $36 \%$ & 0.1185 \\
\hline Female & $29 \%$ & $41 \%$ & 0.1094 \\
\hline Male & $12 \%$ & $15 \%$ & 1.0000 \\
\hline \multicolumn{4}{|c|}{ Anger Expression Index } \\
\hline All Subjects & $33 \%$ & $12 \%$ & $0.0004 *$ \\
\hline Female & $35 \%$ & $12 \%$ & $0.0010^{*}$ \\
\hline Male & $42 \%$ & $31 \%$ & 0.3750 \\
\hline \multicolumn{4}{|c|}{$\begin{array}{l}* \text { indicates significant difference from baseline to after the intervention }(\mathrm{p}<0.05) \\
\text { Note: According to the STAXI manual, scores differentiating }<\text { or } \geq 75^{\text {th }} \text { percentile differ } \\
\text { depending on the sex profile of the group under study (i.e., males and females together, females } \\
\text { only or males only). Appropriate score cut-offs were used for each category in this table. As the } \\
\text { cut-off values vary depending on sex-profile of the group, the group proportion of those scoring } \\
\geq 75^{\text {th }} \text { percentile will not be the average of male and female proportions. }\end{array}$} \\
\hline
\end{tabular}


Table 20. Internal consistency measures using Cronbach's $\alpha$ at baseline and post intervention for trait anger, trait anger temperament, trait anger reaction, anger expression inwards, anger expression outwards, perceived stress, anger control inwards and anger control outwards

\section{Baseline $\alpha$ Coefficient Post intervention $\alpha$ Coefficient}

\begin{tabular}{|lll|}
\hline Trait Anger & 0.88 & 0.85 \\
Trait Anger & 0.89 & 0.85 \\
Temperament & 0.78 & 0.78 \\
Trait Anger Reaction & 0.79 & 0.76 \\
Anger Expression In & 0.74 & 0.62 \\
Anger Expression Out & 0.90 & 0.86 \\
Perceived Stress & 0.92 & 0.89 \\
Anger Control In & 0.87 & 0.89 \\
Anger Control Out & &
\end{tabular}

Only standardized scores for Cronbach's $\alpha$ have been reported. Note: in accordance with Nunnally \& Bernstein's suggested value of 0.70 indicating sufficient internal consistency, no $\mathrm{p}$-values have been assigned to $\alpha$ coefficients. 
Table 21. Changes in 24-hour, daytime and nighttime blood pressure from pre to post intervention

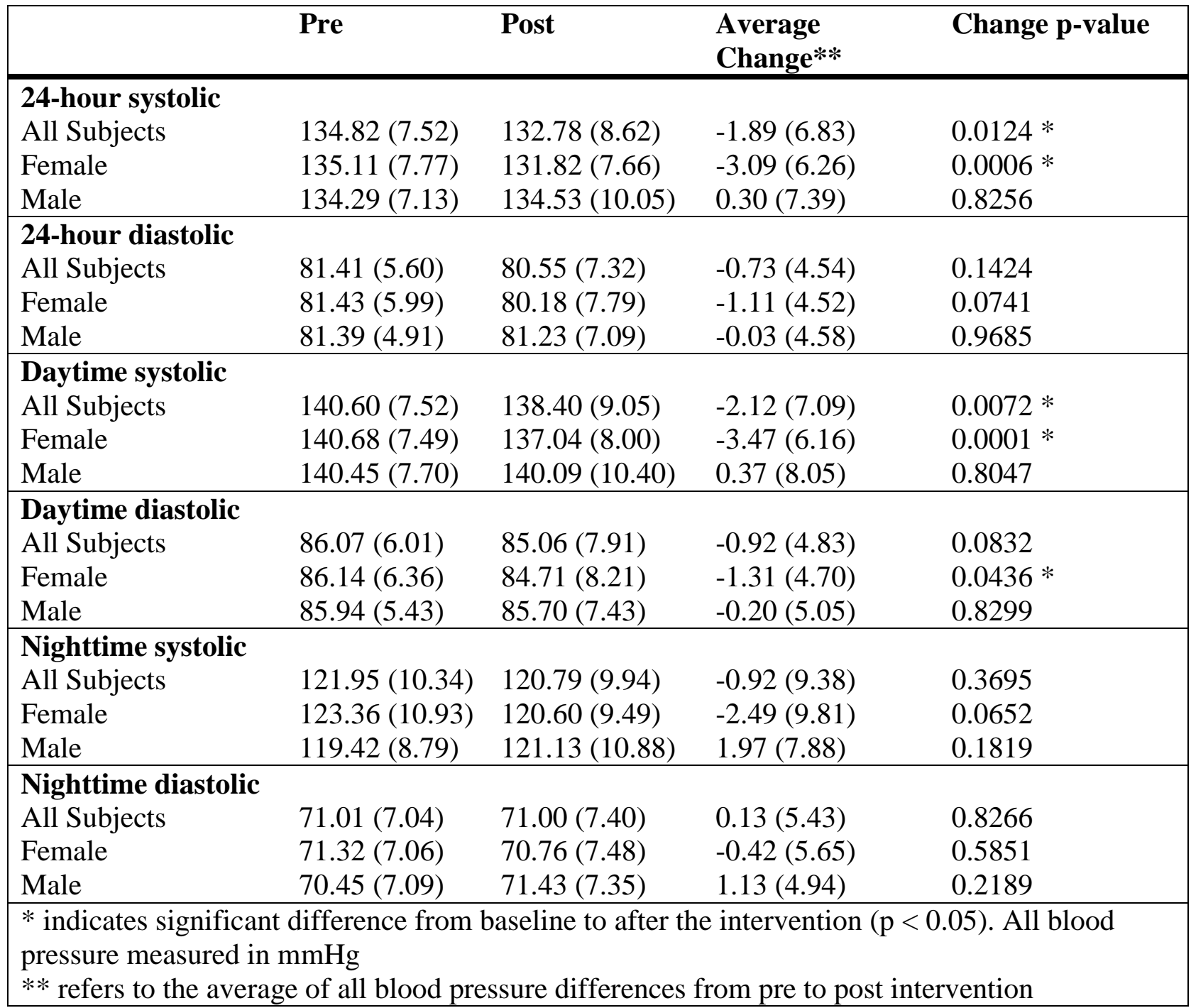


Table 22. Correlations between changes in trait anger, trait anger temperament, trait anger reaction, anger expression inwards, anger expression outwards, perceived stress and change in 24-hour blood pressure from pre to post intervention

\begin{tabular}{|c|c|c|c|c|c|c|}
\hline \multirow[b]{2}{*}{ Anger Measure } & \multicolumn{2}{|c|}{ All Subjects } & \multicolumn{2}{|l|}{ Female } & \multicolumn{2}{|l|}{ Male } \\
\hline & Systolic & Diastolic & Systolic & Diastolic & Systolic & Diastolic \\
\hline T-Ang & -0.043 & -0.008 & 0.043 & 0.010 & -0.088 & -0.001 \\
\hline T-Ang/T & 0.135 & 0.093 & 0.195 & 0.103 & 0.040 & 0.082 \\
\hline T-Ang/R & -0.148 & -0.058 & -0.150 & -0.055 & -0.075 & -0.023 \\
\hline AXI & 0.042 & 0.147 & 0.137 & $0.257 *$ & -0.018 & 0.038 \\
\hline $\mathrm{AXO}$ & 0.041 & 0.021 & 0.152 & -0.002 & -0.156 & 0.037 \\
\hline PS & -0.031 & 0.020 & -0.173 & -0.049 & 0.197 & 0.155 \\
\hline $\begin{array}{l}\text { Note. }{ }^{*} \mathrm{p}<0.10, \\
=\text { Trait Anger } \\
\mathrm{PS}=\text { Perceived }\end{array}$ & $\begin{array}{l}.05 . \mathrm{T}- \\
\mathrm{n}, \mathrm{AXI}\end{array}$ & $\begin{array}{l}\text { Trait A } \\
\text { er Exp }\end{array}$ & T-Ang & $\begin{array}{l}\text { rait An } \\
\mathrm{XO}=\end{array}$ & $\begin{array}{l}\text { emper } \\
\text { Expre }\end{array}$ & $\begin{array}{l}\text { t, T-Ang/R } \\
\text { Outwards, }\end{array}$ \\
\hline
\end{tabular}

Table 23. Correlations between changes in trait anger, trait anger temperament, trait anger reaction, anger expression inwards, anger expression outwards, perceived stress and change in daytime blood pressure from pre to post intervention

\begin{tabular}{|c|c|c|c|c|c|c|}
\hline \multirow[b]{2}{*}{$\begin{array}{l}\text { Anger } \\
\text { Measure }\end{array}$} & \multicolumn{2}{|c|}{ All Subjects } & \multicolumn{2}{|l|}{ Female } & \multicolumn{2}{|l|}{ Male } \\
\hline & Systolic & Diastolic & Systolic & Diastolic & Systolic & Diastolic \\
\hline T-Ang & -0.091 & -0.024 & -0.020 & -0.001 & -0.111 & -0.027 \\
\hline T-Ang/T & 0.148 & 0.073 & 0.220 & 0.072 & 0.047 & 0.024 \\
\hline T-Ang/R & -0.164 & -0.066 & -0.168 & -0.084 & -0.087 & -0.006 \\
\hline AXI & 0.004 & 0.099 & 0.183 & $0.279 *$ & -0.123 & -0.088 \\
\hline $\mathrm{AXO}$ & -0.019 & 0.005 & 0.124 & 0.029 & -0.251 & -0.053 \\
\hline PS & -0.039 & 0.004 & -0.139 & -0.007 & 0.113 & 0.037 \\
\hline
\end{tabular}


Table 24. Correlations between changes in trait anger, trait anger temperament, trait anger reaction, anger expression inwards, anger expression outwards, perceived stress and change in nighttime blood pressure from pre to post intervention

\begin{tabular}{|c|c|c|c|c|c|c|}
\hline \multirow[b]{2}{*}{ Anger Measure } & \multicolumn{2}{|c|}{ All Subjects } & \multicolumn{2}{|l|}{ Female } & \multicolumn{2}{|l|}{ Male } \\
\hline & Systolic & Diastolic & Systolic & Diastolic & Systolic & Diastolic \\
\hline T-Ang & -0.051 & -0.014 & -0.016 & 0.033 & -0.046 & -0.046 \\
\hline T-Ang/T & 0.102 & 0.073 & 0.145 & 0.091 & 0.004 & 0.050 \\
\hline T-Ang/R & -0.176 & -0.065 & -0.217 & -0.023 & -0.013 & -0.077 \\
\hline AXI & 0.061 & 0.141 & -0.009 & 0.126 & 0.221 & 0.208 \\
\hline AXO & 0.093 & -0.007 & 0.132 & -0.041 & -0.035 & 0.026 \\
\hline PS & -0.036 & 0.048 & -0.170 & -0.025 & 0.299 & 0.224 \\
\hline
\end{tabular}


Table 25. Correlations between changes in anger control inwards, anger control outwards, anger expression index and change in 24-hour blood pressure from pre to post intervention

\begin{tabular}{|lllllll|}
\hline & \multicolumn{3}{l}{ All Subjects } & Female & \multicolumn{3}{l|}{ Male } \\
\cline { 2 - 7 } Anger Measure & Systolic & Diastolic & Systolic & Diastolic & Systolic & Diastolic \\
\hline ACI & 0.011 & 0.027 & -0.240 & -0.168 & 0.328 & 0.330 \\
ACO & -0.112 & -0.104 & -0.227 & -0.171 & 0.118 & 0.050 \\
AXX & 0.061 & 0.087 & $0.282 *$ & 0.228 & -0.210 & -0.119 \\
\hline $\begin{array}{l}\text { Note. } \\
\text { AXX }=\text { p Anger Expression Index. }\end{array}$ & & & & & \\
\hline
\end{tabular}

Table 26. Correlations between changes in anger control inwards, anger control outwards, anger expression index and change in daytime blood pressure from pre to post intervention

\begin{tabular}{|lllllll|}
\hline & \multicolumn{3}{l}{ All Subjects } & Female & \multicolumn{3}{l|}{ Male } \\
\cline { 2 - 7 } Anger Measure & Systolic & Diastolic & Systolic & Diastolic & Systolic & Diastolic \\
\hline ACI & 0.051 & 0.073 & $-0.272 *$ & -0.165 & $0.423 * *$ & $0.428 * *$ \\
ACO & -0.113 & -0.071 & $-0.242 *$ & -0.159 & 0.120 & 0.110 \\
AXX & 0.013 & 0.034 & $0.310 * *$ & 0.239 & -0.316 & -0.251 \\
\hline $\begin{array}{l}\text { Note. } \\
\text { AXX }=\text { p Anger Expression Index. }\end{array}$ & & & & & \\
\hline
\end{tabular}

Table 27. Correlations between changes in anger control inwards, anger control outwards, anger expression index and change in nighttime blood pressure from pre to post intervention

\begin{tabular}{|c|c|c|c|c|c|c|}
\hline \multirow[b]{2}{*}{ Anger Measure } & \multicolumn{2}{|c|}{ All Subjects } & \multicolumn{2}{|l|}{ Female } & \multicolumn{2}{|l|}{ Male } \\
\hline & Systolic & Diastolic & Systolic & Diastolic & Systolic & Diastolic \\
\hline ACI & -0.168 & -0.116 & -0.213 & -0.155 & -0.122 & -0.062 \\
\hline $\mathrm{ACO}$ & -0.151 & -0.149 & -0.149 & -0.106 & -0.092 & -0.200 \\
\hline $\mathrm{AXX}$ & 0.171 & 0.152 & 0.183 & 0.140 & 0.160 & 0.178 \\
\hline
\end{tabular}


Table 28. Effects of post intervention trait anger on post intervention 24-hour blood pressure after adjustment for baseline blood pressure, baseline psychosocial functioning and other covariates

\begin{tabular}{|c|c|c|c|c|c|c|c|c|c|c|c|c|}
\hline \multirow[b]{3}{*}{ Predictors } & \multicolumn{4}{|l|}{ All Subjects } & \multicolumn{4}{|l|}{ Females } & \multicolumn{4}{|l|}{ Males } \\
\hline & Systolic & & Diastolic & & Systolic & & Diastolic & & Systolic & & Diastolic & \\
\hline & $\beta(\mathbf{S E})$ & p-value & $\beta(\mathbf{S E})$ & p-value & $\beta$ (SE) & p-value & $\beta$ (SE) & p-value & $\beta$ (SE) & p-value & $\beta$ (SE) & p-value \\
\hline Pre MBSR 24-hour BP** & $0.87(0.13)$ & $<.0001$ & $1.02(0.12)$ & $<.0001$ & $0.97(0.17)$ & $<.0001$ & $0.92(0.14)$ & $<.0001$ & & & $1.26(0.28)$ & 0.001 \\
\hline Pre MBSR T-Ang* & $-0.10(0.27)$ & 0.71 & $-0.08(0.18)$ & 0.64 & $-0.26(0.33)$ & 0.44 & $-0.19(0.23)$ & 0.42 & & & $0.01(0.53)$ & 0.98 \\
\hline Post MBSR T-Ang & $0.11(0.39)$ & 0.78 & $-0.01(0.26)$ & 0.97 & $0.08(0.48)$ & 0.86 & $-0.27(0.34)$ & 0.43 & & & $0.17(0.85)$ & 0.85 \\
\hline Age & $-0.07(0.08)$ & 0.41 & $-0.11(0.05)$ & 0.04 & $-0.22(0.10)$ & 0.04 & $-0.19(0.07)$ & 0.01 & & & $-0.05(0.11)$ & 0.65 \\
\hline BMI* & $0.03(0.18)$ & 0.85 & $-0.14(0.12)$ & 0.23 & $-0.14(0.17)$ & 0.42 & $-0.17(0.11)$ & 0.14 & & & $0.16(0.50)$ & 0.75 \\
\hline Sex (female) & $-2.67(1.95)$ & 0.18 & $-0.28(1.31)$ & 0.83 & & & & & & & & \\
\hline FHx premature CVD (Yes) & $-3.13(1.98)$ & 0.12 & $-3.32(1.33)$ & 0.02 & $-2.88(2.30)$ & 0.22 & $-3.03(1.62)$ & 0.07 & & & $-4.18(3.19)$ & 0.21 \\
\hline Drink $\geq 10$ drinks/wk (Yes)* & $3.43(2.27)$ & 0.14 & $3.28(1.52)$ & 0.04 & $4.73(2.69)$ & 0.09 & $6.02(1.91)$ & 0.004 & & & $-0.10(3.05)$ & 0.97 \\
\hline Relaxation regularly (Yes)* & $-0.82(2.70)$ & 0.76 & $-1.57(1.81)$ & 0.39 & $-1.50(2.33)$ & 0.52 & $-2.78(1.65)$ & 0.10 & & & 0.00 & \\
\hline Race (Causasian) & $-1.18(2.63)$ & 0.66 & $-2.96(1.76)$ & 0.10 & $1.21(2.61)$ & 0.65 & $-2.86(1.85)$ & 0.13 & & & $-1.85(4.60)$ & 0.69 \\
\hline Education (> high school) & $3.15(2.58)$ & 0.23 & $0.94(1.72)$ & 0.59 & $2.60(2.62)$ & 0.33 & $1.61(1.85)$ & 0.39 & & & $3.75(7.67)$ & 0.63 \\
\hline Regular Exercise (Yes)* & $-5.41(2.94)$ & 0.07 & $-2.83(1.96)$ & 0.16 & $-2.02(2.98)$ & 0.50 & $-1.37(2.07)$ & 0.51 & & & $-6.67(5.62)$ & 0.26 \\
\hline
\end{tabular}

* Baseline covariates. NOTE1: Grey fill indicates an overall null model. NOTE2: only 1 male subject regularly practiced relaxation, thus, that $\beta$ coefficient is zero for males. Family history (FHx), cardiovascular disease (CVD).

Table 29. Effects of post intervention trait anger on post intervention daytime blood pressure after adjustment for baseline blood pressure, baseline psychosocial functioning and other covariates

\begin{tabular}{|c|c|c|c|c|c|c|c|c|c|c|c|}
\hline \multirow[b]{3}{*}{ Predictors } & \multicolumn{4}{|l|}{ All Subjects } & \multicolumn{4}{|l|}{ Females } & \multicolumn{3}{|l|}{ Males } \\
\hline & Systolic & & Diastolic & & Systolic & & Diastolic & & Systolic & & Diastolic \\
\hline & $\beta(\mathbf{S E})$ & p-value & $\beta(\mathbf{S E})$ & p-value & $\beta(\mathbf{S E})$ & p-value & $\beta(\mathbf{S E})$ & p-value & $\beta$ (SE) & p-value & p-value \\
\hline Pre MBSR daytime BP* & $0.78(0.13)$ & $<.0001$ & $0.89(0.11)$ & $<.0001$ & $0.90(0.16)$ & $<.0001$ & $0.83(0.12)$ & $<.0001$ & & & \\
\hline Pre MBSR T-Ang* & $-0.03(0.28)$ & 0.93 & $-0.06(0.19)$ & 0.75 & $-0.12(0.35)$ & 0.74 & $-0.10(0.24)$ & 0.67 & & & \\
\hline Post MBSR T-Ang & $-0.03(0.41)$ & 0.93 & $-0.10(0.28)$ & 0.73 & $-0.15(0.51)$ & 0.76 & $-0.53(0.35)$ & 0.15 & & & \\
\hline Age & $-0.09(0.09)$ & 0.30 & $-0.15(0.06)$ & 0.01 & $-0.23(0.11)$ & 0.04 & $-0.24(0.07)$ & 0.002 & & & \\
\hline BMI* & $0.06(0.18)$ & 0.73 & $-0.11(0.13)$ & 0.40 & $-0.06(0.17)$ & 0.73 & $-0.12(0.12)$ & 0.30 & & & \\
\hline Sex (female) & $-4.52(2.03)$ & 0.03 & $-1.21(1.39)$ & 0.39 & & & & & & & \\
\hline FHx premature CVD (Yes) & $-2.77(2.07)$ & 0.19 & $-3.01(1.42)$ & 0.04 & $-3.94(2.46)$ & 0.12 & $-3.93(1.68)$ & 0.03 & & & \\
\hline Drink $\geq 10$ drinks/wk (Yes)* & $2.98(2.37)$ & 0.21 & $3.19(1.62)$ & 0.055 & $2.52(2.89)$ & 0.39 & $4.71(2.00)$ & 0.03 & & & \\
\hline Relaxation regularly (Yes)* & $1.55(2.82)$ & 0.58 & $-0.10(1.94)$ & 0.96 & $1.85(2.48)$ & 0.46 & $-0.85(1.71)$ & 0.62 & & & \\
\hline Race (Causasian) & $-2.03(2.74)$ & 0.46 & $-3.05(1.89)$ & 0.11 & $0.82(2.76)$ & 0.77 & $-2.43(1.91)$ & 0.21 & & & \\
\hline Education (> high school) & $2.09(2.69)$ & 0.44 & $1.03(1.83)$ & 0.58 & $2.60(2.79)$ & 0.36 & $2.41(1.92)$ & 0.22 & & & \\
\hline Regular Exercise (Yes)* & $-5.37(3.08)$ & 0.09 & $-3.58(2.10)$ & 0.10 & $-1.49(3.12)$ & 0.64 & $-1.42(2.17)$ & 0.52 & & & \\
\hline
\end{tabular}

* Baseline covariates. NOTE1: Grey fill indicates an overall null model. Family history (FHx), cardiovascular disease (CVD). 
Table 30. Effects of post intervention trait anger temperament on post intervention 24-hour blood pressure after adjustment for baseline blood pressure, baseline psychosocial functioning and other covariates

\begin{tabular}{|c|c|c|c|c|c|c|c|c|c|c|c|}
\hline \multirow[b]{3}{*}{ Predictors } & \multicolumn{4}{|l|}{ All Subjects } & \multicolumn{4}{|l|}{ Females } & \multicolumn{3}{|l|}{ Males } \\
\hline & Systolic & & Diastolic & & Systolic & & Diastolic & & Systolic & Diastolic & \\
\hline & $\beta(\mathbf{S E})$ & p-value & $\beta(\mathbf{S E})$ & p-value & $\beta(\mathbf{S E})$ & p-value & $\beta(\mathbf{S E})$ & p-value & p-value & $\beta(\mathbf{S E})$ & p-value \\
\hline Pre MBSR 24-hour BP* & $0.89(0.13)$ & $<.0001$ & $1.00(0.12)$ & $<.0001$ & $1.05(0.16)$ & $<.0001$ & $0.87(0.15)$ & $<.0001$ & & $1.22(0.28)$ & 0.001 \\
\hline Pre MBSR T-Ang/T* & $-0.71(0.55)$ & 0.21 & $-0.45(0.38)$ & 0.24 & $-1.76(0.62)$ & 0.01 & $-0.77(0.52)$ & 0.15 & & $-0.66(0.93)$ & 0.50 \\
\hline Post MBSR T-Ang/T & $1.14(0.82)$ & 0.17 & $0.40(0.56)$ & 0.47 & $2.71(0.95)$ & 0.01 & $0.47(0.80)$ & 0.56 & & $1.33(1.56)$ & 0.41 \\
\hline Age & $-0.08(0.08)$ & 0.34 & $-0.11(0.05)$ & 0.04 & $-0.29(0.10)$ & 0.01 & $-0.19(0.07)$ & 0.01 & & $-0.04(0.11)$ & 0.73 \\
\hline BMI* & $0.04(0.17)$ & 0.81 & $-0.13(0.12)$ & 0.26 & $-0.21(0.15)$ & 0.17 & $-0.19(0.12)$ & 0.11 & & $0.18(0.40)$ & 0.66 \\
\hline Sex (female) & $-2.10(1.95)$ & 0.29 & $-0.12(1.32)$ & 0.93 & & & & & & & \\
\hline FHx premature CVD (Yes) & $-3.05(1.93)$ & 0.12 & $-3.24(1.31)$ & 0.02 & $-1.63(2.00)$ & 0.42 & $-2.33(1.64)$ & 0.17 & & $-3.91(3.03)$ & 0.22 \\
\hline Drink $\geq 10$ drinks/wk $(Y e s)^{*}$ & $3.64(2.26)$ & 0.11 & $3.52(1.53)$ & 0.03 & $5.32(2.36)$ & 0.03 & $5.97(1.95)$ & 0.005 & & $-0.57(3.17)$ & 0.86 \\
\hline Relaxation regularly (Yes)* & $-1.38(2.69)$ & 0.61 & $-1.86(1.82)$ & 0.31 & $-2.97(2.15)$ & 0.18 & $-2.98(1.77)$ & 0.10 & & 0.00 & \\
\hline Race (Causasian) & $-1.82(2.60)$ & 0.49 & $-3.33(1.76)$ & 0.06 & $-0.05(2.29)$ & 0.98 & $-3.30(1.88)$ & 0.09 & & $-2.59(4.55)$ & 0.58 \\
\hline Education (> high school) & $2.84(2.56)$ & 0.27 & $0.66(1.72)$ & 0.70 & $-1.34(2.58)$ & 0.61 & $-0.19(2.13)$ & 0.93 & & $6.11(6.90)$ & 0.39 \\
\hline Regular Exercise (Yes)* & $-5.35(2.88)$ & 0.07 & $-2.89(1.95)$ & 0.14 & $-3.23(2.67)$ & 0.24 & $-1.58(2.16)$ & 0.47 & & $-6.93(5.09)$ & 0.20 \\
\hline
\end{tabular}

* Baseline covariates. NOTE1: Grey fill indicates an overall null model. NOTE2: only 1 male subject regularly practiced relaxation, thus, that $\beta$ coefficient is zero for males. Family history (FHx), cardiovascular disease (CVD).

Table 31. Effects of post intervention trait anger temperament on post intervention daytime blood pressure after adjustment for baseline blood pressure, baseline psychosocial functioning and other covariates

\begin{tabular}{|c|c|c|c|c|c|c|c|c|c|c|c|c|}
\hline \multirow[b]{3}{*}{ Predictors } & \multicolumn{4}{|l|}{ All Subjects } & \multicolumn{4}{|l|}{ Females } & \multicolumn{4}{|l|}{ Males } \\
\hline & Systolic & & Diastolic & & Systolic & & Diastolic & & Systolic & & Diastolic & \\
\hline & $\beta(\mathbf{S E})$ & p-value & $\beta(\mathbf{S E})$ & p-value & $\beta(\mathbf{S E})$ & p-value & $\beta(\mathrm{SE})$ & p-value & $\beta(\mathbf{S E})$ & p-value & $\beta(\mathbf{S E})$ & p-value \\
\hline Pre MBSR daytime BP* & $0.79(0.13)$ & $<.0001$ & $0.87(0.11)$ & $<.0001$ & $0.95(0.16)$ & $<.0001$ & $0.80(0.14)$ & $<.0001$ & & & & \\
\hline Pre MBSR T-Ang/T** & $-0.56(0.58)$ & 0.34 & $-0.42(0.41)$ & 0.30 & $-1.47(0.70)$ & 0.04 & $-0.62(0.56)$ & 0.28 & & & & \\
\hline Post MBSR T-Ang/T & $0.77(0.86)$ & 0.38 & $0.31(0.60)$ & 0.61 & $2.05(1.07)$ & 0.07 & $0.04(0.85)$ & 0.96 & & & & \\
\hline Age & $-0.09(0.09)$ & 0.28 & $-0.14(0.06)$ & 0.02 & $-0.28(0.10)$ & 0.01 & $-0.23(0.08)$ & 0.01 & & & & \\
\hline BMI* & $0.07(0.18)$ & 0.70 & $-0.10(0.13)$ & 0.44 & $-0.12(0.16)$ & 0.48 & $-0.15(0.13)$ & 0.26 & & & & \\
\hline Sex (female) & $-4.15(2.06)$ & 0.049 & $-1.08(1.41)$ & 0.45 & & & & & & & & \\
\hline FHx premature CVD (Yes) & $-2.63(2.04)$ & 0.20 & $-2.91(1.40)$ & 0.04 & $-2.58(2.27)$ & 0.27 & $-3.06(1.77)$ & 0.09 & & & & \\
\hline Drink $\geq 10$ drinks/wk (Yes)* & $3.27(2.39)$ & 0.18 & $3.46(1.64)$ & 0.04 & $3.10(2.68)$ & 0.26 & $4.73(2.12)$ & 0.03 & & & & \\
\hline Relaxation regularly (Yes)* & $1.12(2.83)$ & 0.69 & $-0.34(1.97)$ & 0.86 & $0.64(2.42)$ & 0.79 & $-0.93(1.92)$ & 0.63 & & & & \\
\hline Race (Causasian) & $-2.60(2.73)$ & 0.35 & $-3.48(1.90)$ & 0.07 & $-0.42(2.56)$ & 0.87 & $-3.11(2.01)$ & 0.13 & & & & \\
\hline Education (> high school) & $1.80(2.70)$ & 0.51 & $0.77(1.84)$ & 0.68 & $-1.06(2.92)$ & 0.72 & $0.58(2.30)$ & 0.80 & & & & \\
\hline Regular Exercise (Yes)* & $-5.41(3.05)$ & 0.08 & $-3.66(2.09)$ & 0.09 & $-2.42(2.97)$ & 0.42 & $-1.62(2.35)$ & 0.50 & & & & \\
\hline
\end{tabular}

* Baseline covariates. NOTE1: Grey fill indicates an overall null model. Family history (FHx), cardiovascular disease (CVD). 
Table 32. Effects of post intervention trait anger temperament as a multilevel categorical predictor variable divided into quartiles on post intervention 24-hour blood pressure after adjustment for baseline blood pressure, baseline psychosocial functioning and other covariates

\begin{tabular}{|c|c|c|c|c|c|c|c|c|c|c|c|}
\hline \multirow[b]{3}{*}{ Predictors } & \multicolumn{4}{|l|}{ All Subjects } & \multicolumn{4}{|l|}{ Females } & \multicolumn{3}{|l|}{ Males } \\
\hline & Systolic & & Diastolic & & Systolic & & Diastolic & & Systolic & Diastolic & \\
\hline & $\beta$ (SE) & p-value & $\beta(\mathbf{S E})$ & p-value & $\beta$ (SE) & p-value & $\beta$ (SE) & p-value & p-value & $\beta(\mathbf{S E})$ & p-value \\
\hline Pre MBSR 24-hour BP* & $0.89(0.13)$ & $<.0001$ & $1.00(0.12)$ & $<.0001$ & $1.01(0.17)$ & $<.0001$ & $0.92(0.16)$ & $<.0001$ & & $1.67(0.28)$ & 0.0001 \\
\hline Pre MBSR T-Ang/T* & $-0.64(0.58)$ & 0.28 & $-0.40(0.39)$ & 0.31 & $-1.57(0.62)$ & 0.02 & $-0.83(0.49)$ & 0.11 & & $0.67(0.85)$ & 0.45 \\
\hline \multicolumn{12}{|l|}{ Post MBSR T-Ang/T } \\
\hline Q1 vs Q4 & $-5.11(3.78)$ & 0.18 & $-1.81(2.54)$ & 0.48 & $-9.81(3.89)$ & 0.02 & $-2.16(3.09)$ & 0.49 & & $0.26(6.77)$ & 0.97 \\
\hline Q2 vs Q4 & $-2.89(4.01)$ & 0.48 & $-1.15(2.71)$ & 0.67 & $-9.69(4.27)$ & 0.03 & $-3.55(3.38)$ & 0.30 & & $10.17(6.64)$ & 0.16 \\
\hline Q3 vs Q4 & $-2.64(3.24)$ & 0.42 & $-2.06(2.19)$ & 0.35 & $-5.81(3.08)$ & 0.07 & $-2.31(2.42)$ & 0.35 & & $0.96(6.08)$ & 0.88 \\
\hline Age & $-0.09(0.08)$ & 0.30 & $-0.11(0.05)$ & 0.04 & $-0.25(0.10)$ & 0.02 & $-0.19(0.07)$ & 0.02 & & $-0.10(0.10)$ & 0.32 \\
\hline BMI* & $0.03(0.18)$ & 0.88 & $-0.15(0.12)$ & 0.23 & $-0.18(0.16)$ & 0.28 & $-0.20(0.12)$ & 0.12 & & $0.35(0.39)$ & 0.40 \\
\hline Sex (female) & $-2.31(1.99)$ & 0.25 & $-0.06(1.35)$ & 0.96 & & & & & & & \\
\hline FHx premature CVD (Yes) & $-3.10(1.99)$ & 0.13 & $-3.43(1.35)$ & 0.01 & $-2.07(2.14)$ & 0.34 & $-2.66(1.68)$ & 0.13 & & $-6.77(2.75)$ & 0.03 \\
\hline Drink $\geq 10$ drinks/wk (Yes)* & $3.49(2.53)$ & 0.17 & $3.15(1.71)$ & 0.07 & $6.77(2.89)$ & 0.03 & $6.44(2.30)$ & 0.01 & & $-2.33(3.49)$ & 0.52 \\
\hline Relaxation regularly (Yes)* & $-1.05(2.85)$ & 0.71 & $-1.55(1.92)$ & 0.42 & $-3.11(2.37)$ & 0.20 & $-3.20(1.89)$ & 0.10 & & 0.00 & \\
\hline Race (Causasian) & $-1.37(2.85)$ & 0.63 & $-2.92(1.93)$ & 0.14 & $-0.32(2.59)$ & 0.90 & $-3.66(2.09)$ & 0.09 & & $7.10(5.04)$ & 0.19 \\
\hline Education (> high school) & $2.48(2.67)$ & 0.36 & $0.76(1.79)$ & 0.68 & $0.04(2.57)$ & 0.99 & $0.13(2.02)$ & 0.95 & & $3.79(5.13)$ & 0.48 \\
\hline Regular Exercise (Yes)* & $-5.23(2.99)$ & 0.09 & $-2.85(2.01)$ & 0.16 & $-4.02(3.02)$ & 0.20 & $-2.25(2.33)$ & 0.34 & & $-7.82(4.29)$ & 0.10 \\
\hline
\end{tabular}

* Baseline covariates. NOTE1: Grey fill indicates an overall null model. NOTE2: only 1 male subject regularly practiced relaxation, thus, that $\beta$ coefficient is zero for males. Family history (FHx), cardiovascular disease (CVD). Q1= bottom $25^{\text {th }}$ percent quartile, Q2 $=$ second quartile, $\mathrm{Q} 3=$ third quartile, $\mathrm{Q} 4=$ top $25^{\text {th }}$ percent quartile. 
Table 33. Effects of post intervention trait anger temperament as a multilevel categorical predictor variable divided into quartiles on post intervention daytime blood pressure after adjustment for baseline blood pressure, baseline psychosocial functioning and other covariates

\begin{tabular}{|c|c|c|c|c|c|c|c|c|c|c|c|}
\hline \multirow[b]{3}{*}{ Predictors } & \multicolumn{4}{|l|}{ All Subjects } & \multicolumn{4}{|l|}{ Females } & \multicolumn{3}{|l|}{ Males } \\
\hline & Systolic & & Diastolic & & Systolic & & Diastolic & & Systolic & Diastolic & \\
\hline & $\beta(\mathbf{S E})$ & p-value & $\beta(\mathbf{S E})$ & p-value & $\beta(\mathbf{S E})$ & p-value & $\beta(\mathbf{S E})$ & p-value & p-value & $\beta(\mathbf{S E})$ & p-value \\
\hline Pre MBSR daytime BP* & $0.79(0.13)$ & $<.0001$ & $0.87(0.11)$ & $<.0001$ & $0.94(0.16)$ & $<.0001$ & $0.86(0.14)$ & $<.0001$ & & $1.59(0.26)$ & 0.0001 \\
\hline Pre MBSR T-Ang/T* & $-0.40(0.61)$ & 0.51 & $-0.41(0.42)$ & 0.34 & $-1.48(0.68)$ & 0.04 & $-0.91(0.52)$ & 0.09 & & $1.01(0.90)$ & 0.29 \\
\hline \multicolumn{12}{|l|}{ Post MBSR T-Ang/T } \\
\hline Q1 vs Q4 & $-2.95(3.95)$ & 0.46 & $-1.52(2.74)$ & 0.58 & $-8.11(4.27)$ & 0.07 & $-1.77(3.25)$ & 0.59 & & $0.43(7.02)$ & 0.95 \\
\hline Q2 vs Q4 & $-0.15(4.21)$ & 0.97 & $-0.97(2.91)$ & 0.74 & $-9.06(4.75)$ & 0.07 & $-4.40(3.59)$ & 0.23 & & $13.40(7.02)$ & 0.09 \\
\hline Q3 vs Q4 & $-0.59(3.40)$ & 0.86 & $-1.54(2.36)$ & 0.52 & $-4.17(3.39)$ & 0.23 & $-2.37(2.55)$ & 0.36 & & $-0.68(6.29)$ & 0.92 \\
\hline Age & $-0.10(0.09)$ & 0.27 & $-0.15(0.06)$ & 0.02 & $-0.26(0.10)$ & 0.02 & $-0.22(0.08)$ & 0.01 & & $-0.12(0.10)$ & 0.27 \\
\hline BMI* & $0.06(0.19)$ & 0.73 & $-0.11(0.13)$ & 0.41 & $-0.08(0.17)$ & 0.66 & $-0.15(0.13)$ & 0.27 & & $0.53(0.42)$ & 0.23 \\
\hline Sex (female) & $-4.49(2.09)$ & 0.04 & $-1.04(1.44)$ & 0.47 & & & & & & & \\
\hline FHx premature CVD (Yes) & $-2.54(2.09)$ & 0.23 & $-3.04(1.45)$ & 0.04 & $-2.86(2.37)$ & 0.24 & $-3.32(1.77)$ & 0.07 & & $-6.01(2.84)$ & 0.06 \\
\hline Drink $>10$ drinks/wk (Yes)* & $2.98(2.66)$ & 0.27 & $3.21(1.84)$ & 0.09 & $5.12(3.18)$ & 0.12 & $5.52(2.42)$ & 0.03 & & $-3.22(3.72)$ & 0.41 \\
\hline Relaxation regularly (Yes)* & $1.60(2.99)$ & 0.59 & $-0.13(2.08)$ & 0.95 & $0.01(2.59)$ & 1.00 & $-1.43(1.98)$ & 0.48 & & 0.00 & \\
\hline Race (Causasian) & $-1.92(2.99)$ & 0.52 & $-3.22(2.08)$ & 0.13 & $-1.32(2.83)$ & 0.64 & $-3.86(2.17)$ & 0.09 & & $8.26(5.40)$ & 0.16 \\
\hline Education (> high school) & $1.44(2.80)$ & 0.61 & $0.81(1.93)$ & 0.67 & $-0.17(2.81)$ & 0.95 & $0.45(2.13)$ & 0.83 & & $4.24(5.38)$ & 0.45 \\
\hline Regular Exercise (Yes)* & $-4.99(3.16)$ & 0.12 & $-3.63(2.17)$ & 0.10 & $-3.67(3.30)$ & 0.28 & $-2.70(2.46)$ & 0.28 & & $-8.11(4.43)$ & 0.10 \\
\hline
\end{tabular}

* Baseline covariates. NOTE1: Grey fill indicates an overall null model. NOTE2: only 1 male subject regularly practiced relaxation, thus, that $\beta$ coefficient is zero for males. Family history $(\mathrm{FHx})$, cardiovascular disease (CVD). Q1 $=$ bottom $25^{\text {th }}$ percent quartile, Q2 $=$ second quartile, $\mathrm{Q} 3=$ third quartile, $\mathrm{Q} 4=$ top $25^{\text {th }}$ percent quartile. 
Table 34. Effects of post intervention trait anger reaction on post intervention 24-hour blood pressure after adjustment for baseline blood pressure, baseline psychosocial functioning and other covariates

\begin{tabular}{|c|c|c|c|c|c|c|c|c|c|c|c|}
\hline \multirow[b]{3}{*}{ Predictors } & \multicolumn{4}{|l|}{ All Subjects } & \multicolumn{4}{|l|}{ Females } & \multicolumn{3}{|l|}{ Males } \\
\hline & Systolic & & Diastolic & & Systolic & & Diastolic & & Systolic & Diastolic & \\
\hline & $\beta(\mathrm{SE})$ & p-value & $\beta(\mathbf{S E})$ & p-value & $\beta(\mathrm{SE})$ & p-value & $\beta(\mathbf{S E})$ & p-value & p-value & $\beta$ (SE) & p-value \\
\hline Pre MBSR 24-hour BP* & $0.86(0.13)$ & $<.0001$ & $1.02(0.12)$ & $<.0001$ & $1.01(0.18)$ & $<.0001$ & $0.91(0.15)$ & $<.0001$ & & $1.33(0.27)$ & 0.0004 \\
\hline Pre MBSR T-Ang/R* & $-0.08(0.51)$ & 0.88 & $-0.11(0.34)$ & 0.75 & $-0.19(0.52)$ & 0.71 & $-0.45(0.38)$ & 0.24 & & $1.01(1.03)$ & 0.35 \\
\hline Post MBSR T-Ang/R & $0.09(0.61)$ & 0.88 & $0.04(0.41)$ & 0.92 & $-0.58(0.64)$ & 0.37 & $-0.36(0.46)$ & 0.44 & & $-0.78(1.28)$ & 0.55 \\
\hline Age & $-0.07(0.09)$ & 0.44 & $-0.11(0.06)$ & 0.06 & $-0.25(0.11)$ & 0.03 & $-0.19(0.07)$ & 0.01 & & $-0.06(0.11)$ & 0.57 \\
\hline BMI* & $0.06(0.20)$ & 0.76 & $-0.12(0.13)$ & 0.35 & $-0.15(0.19)$ & 0.46 & $-0.13(0.13)$ & 0.33 & & $0.49(0.54)$ & 0.38 \\
\hline Sex (female) & $-2.68(1.99)$ & 0.18 & $-0.23(1.34)$ & 0.86 & & & & & & & \\
\hline FHx premature CVD (Yes) & $-3.27(2.01)$ & 0.11 & $-3.42(1.35)$ & 0.01 & $-3.70(2.26)$ & 0.11 & $-3.39(1.63)$ & 0.048 & & $-3.88(3.11)$ & 0.24 \\
\hline Drink $\geq 10$ drinks/wk $(Y e s)^{*}$ & $3.25(2.27)$ & 0.16 & $3.06(1.53)$ & 0.052 & $4.74(2.68)$ & 0.09 & $6.17(1.95)$ & 0.004 & & $-0.11(2.96)$ & 0.97 \\
\hline Relaxation regularly (Yes)* & $-0.67(2.73)$ & 0.81 & $-1.37(1.83)$ & 0.46 & $-1.58(2.30)$ & 0.50 & $-2.47(1.67)$ & 0.15 & & 0.00 & \\
\hline Race (Causasian) & $-1.07(2.63)$ & 0.69 & $-2.95(1.77)$ & 0.10 & $1.81(2.53)$ & 0.48 & $-2.70(1.86)$ & 0.16 & & $-3.15(4.70)$ & 0.52 \\
\hline Education (> high school) & $3.34(2.66)$ & 0.22 & $1.18(1.79)$ & 0.51 & $3.78(2.61)$ & 0.16 & $2.39(1.90)$ & 0.22 & & $0.63(6.13)$ & 0.92 \\
\hline Regular Exercise (Yes)* & $-5.28(2.97)$ & 0.08 & $-2.69(1.99)$ & 0.18 & $-2.32(2.98)$ & 0.44 & $-1.80(2.11)$ & 0.40 & & $-5.07(5.20)$ & 0.35 \\
\hline
\end{tabular}

* Baseline covariates. NOTE1: Grey fill indicates an overall null model. NOTE2: only 1 male subject regularly practiced relaxation, thus, that $\beta$ coefficient is zero for males. Family history (FHx), cardiovascular disease (CVD).

Table 35. Effects of post intervention trait anger reaction on post intervention daytime blood pressure after adjustment for baseline blood pressure, baseline psychosocial functioning and other covariates

\begin{tabular}{|c|c|c|c|c|c|c|c|c|c|c|c|}
\hline \multirow[b]{3}{*}{ Predictors } & \multicolumn{4}{|l|}{ All Subjects } & \multicolumn{4}{|l|}{ Females } & \multicolumn{3}{|l|}{ Males } \\
\hline & Systolic & & Diastolic & & Systolic & & Diastolic & & Systolic & Diastolic & \\
\hline & $\beta$ (SE) & p-value & $\beta(\mathbf{S E})$ & p-value & $\beta(\mathrm{SE})$ & p-value & $\beta(\mathbf{S E})$ & p-value & p-value & $\beta(\mathbf{S E})$ & p-value \\
\hline Pre MBSR daytime BP* & $0.77(0.13)$ & $<.0001$ & $0.90(0.11)$ & $<.0001$ & $0.95(0.16)$ & $<.0001$ & $0.82(0.13)$ & $<.0001$ & & $1.14(0.27)$ & 0.001 \\
\hline Pre MBSR T-Ang/R* & $-0.01(0.53)$ & 0.98 & $-0.10(0.36)$ & 0.78 & $-0.09(0.55)$ & 0.87 & $-0.40(0.40)$ & 0.32 & & $1.08(1.19)$ & 0.38 \\
\hline Post MBSR T-Ang/R & $0.08(0.63)$ & 0.90 & $-0.04(0.44)$ & 0.92 & $-0.71(0.68)$ & 0.31 & $-0.62(0.48)$ & 0.21 & & $-0.86(1.47)$ & 0.57 \\
\hline Age & $-0.08(0.09)$ & 0.38 & $-0.14(0.06)$ & 0.02 & $-0.26(0.11)$ & 0.03 & $-0.23(0.07)$ & 0.003 & & $-0.08(0.12)$ & 0.50 \\
\hline BMI* & $0.12(0.20)$ & 0.57 & $-0.08(0.14)$ & 0.58 & $-0.01(0.20)$ & 0.95 & $-0.07(0.14)$ & 0.63 & & $0.53(0.62)$ & 0.41 \\
\hline Sex (female) & $-4.48(2.07)$ & 0.04 & $-1.10(1.42)$ & 0.44 & & & & & & & \\
\hline FHx premature CVD (Yes) & $-2.94(2.09)$ & 0.17 & $-3.11(1.44)$ & 0.04 & $-4.80(2.40)$ & 0.06 & $-4.23(1.71)$ & 0.02 & & $-2.36(3.45)$ & 0.51 \\
\hline Drink $\geq 10$ drinks/wk (Yes)* & $2.76(2.37)$ & 0.25 & $2.93(1.63)$ & 0.08 & $2.50(2.84)$ & 0.39 & $4.99(2.05)$ & 0.02 & & $0.75(3.35)$ & 0.83 \\
\hline Relaxation regularly (Yes)* & $1.78(2.84)$ & 0.53 & $0.11(1.97)$ & 0.96 & $1.83(2.41)$ & 0.46 & $-0.61(1.74)$ & 0.73 & & 0.00 & . \\
\hline Race (Causasian) & $-2.09(2.73)$ & 0.45 & $-3.09(1.89)$ & 0.11 & $1.19(2.66)$ & 0.66 & $-2.45(1.92)$ & 0.21 & & $-5.20(5.17)$ & 0.33 \\
\hline Education ( $>$ high school) & $2.46(2.76)$ & 0.38 & $1.34(1.91)$ & 0.49 & $3.77(2.74)$ & 0.18 & $3.06(1.98)$ & 0.13 & & $-0.21(6.98)$ & 0.98 \\
\hline Regular Exercise (Yes)* & $-5.17(3.10)$ & 0.10 & $-3.42(2.13)$ & 0.12 & $-1.75(3.10)$ & 0.58 & $-1.98(2.24)$ & 0.38 & & $-5.52(5.88)$ & 0.37 \\
\hline
\end{tabular}

* Baseline covariates. NOTE1: Grey fill indicates an overall null model. NOTE2: only 1 male subject regularly practiced relaxation, thus,

that $\beta$ coefficient is zero for males. Family history (FHx), cardiovascular disease (CVD). 
Table 36. Effects of post intervention anger expressed inward on post intervention 24-hour blood pressure after adjustment for baseline blood pressure, baseline psychosocial functioning and other covariates

\begin{tabular}{|c|c|c|c|c|c|c|c|c|c|c|c|}
\hline \multirow[b]{3}{*}{ Predictors } & \multicolumn{4}{|l|}{ All Subjects } & \multicolumn{4}{|l|}{ Females } & \multicolumn{3}{|l|}{ Males } \\
\hline & Systolic & & Diastolic & & Systolic & & Diastolic & & Systolic & Diastolic & \\
\hline & $\beta$ (SE) & p-value & $\beta$ (SE) & p-value & $\beta$ (SE) & p-value & $\beta$ (SE) & p-value & p-value & $\beta$ (SE) & p-value \\
\hline Pre MBSR 24-hour BP* & $0.87(0.13)$ & $<.0001$ & $1.01(0.12)$ & $<.0001$ & 0.92 & $<.0001$ & $0.92(0.15)$ & $<.0001$ & & $1.25(0.27)$ & 0.001 \\
\hline Pre MBSR AXI* & $0.12(0.22)$ & 0.58 & $-0.02(0.15)$ & 0.90 & 0.11 & 0.67 & $-0.01(0.19)$ & 0.94 & & $0.21(0.35)$ & 0.57 \\
\hline Post MBSR AXI & $0.30(0.24)$ & 0.23 & $0.21(0.17)$ & 0.21 & 0.37 & 0.21 & $0.36(0.22)$ & 0.11 & & $-0.17(0.35)$ & 0.64 \\
\hline Age & $-0.05(0.08)$ & 0.52 & $-0.10(0.05)$ & 0.07 & -0.15 & 0.14 & $-0.12(0.07)$ & 0.10 & & $-0.03(0.11)$ & 0.77 \\
\hline BMI* & $0.04(0.17)$ & 0.80 & $-0.13(0.12)$ & 0.26 & -0.09 & 0.60 & $-0.14(0.12)$ & 0.23 & & $0.26(0.42)$ & 0.55 \\
\hline Sex (female) & $-2.36(1.90)$ & 0.22 & $-0.07(1.30)$ & 0.96 & & & & & & & \\
\hline FHx premature CVD (Yes) & $-3.65(1.95)$ & 0.07 & $-3.50(1.33)$ & 0.01 & -3.89 & 0.08 & $-3.30(1.64)$ & 0.054 & & $-4.04(3.07)$ & 0.21 \\
\hline Drink $\geq 10$ drinks/wk (Yes)* & $2.81(2.20)$ & 0.21 & $2.93(1.51)$ & 0.06 & 3.84 & 0.13 & $5.02(1.91)$ & 0.01 & & $-1.02(3.69)$ & 0.79 \\
\hline Relaxation regularly (Yes)* & $0.40(2.74)$ & 0.88 & $-0.99(1.87)$ & 0.60 & 0.37 & 0.88 & $-1.22(1.78)$ & 0.50 & & 0.00 & \\
\hline Race (Causasian) & $-0.40(2.56)$ & 0.88 & $-2.60(1.74)$ & 0.14 & 1.17 & 0.63 & $-3.07(1.87)$ & 0.11 & & $-2.03(5.42)$ & 0.71 \\
\hline Education (> high school) & $2.75(2.53)$ & 0.28 & $0.89(1.71)$ & 0.60 & 2.23 & 0.35 & $0.82(1.79)$ & 0.65 & & $1.94(4.97)$ & 0.70 \\
\hline Regular Exercise (Yes)* & $-5.00(2.84)$ & 0.08 & $-2.42(1.93)$ & 0.22 & -1.06 & 0.72 & $-1.13(2.16)$ & 0.60 & & $-7.62(5.30)$ & 0.18 \\
\hline
\end{tabular}

* Baseline covariates. NOTE1: Grey fill indicates an overall null model. NOTE2: only 1 male subject regularly practiced relaxation, thus, that $\beta$ coefficient is zero for males. Family history (FHx), cardiovascular disease (CVD).

Table 37. Effects of post intervention anger expressed inward on post intervention daytime blood pressure after adjustment for baseline blood pressure, baseline psychosocial functioning and other covariates

\begin{tabular}{|c|c|c|c|c|c|c|c|c|c|c|c|}
\hline \multirow[b]{3}{*}{ Predictors } & \multicolumn{4}{|l|}{ All Subjects } & \multicolumn{4}{|l|}{ Females } & \multicolumn{3}{|l|}{ Males } \\
\hline & Systolic & & Diastolic & & Systolic & & Diastolic & & Systolic & Diastolic & \\
\hline & $\beta(\mathrm{SE})$ & p-value & $\beta(\mathbf{S E})$ & p-value & $\beta(\mathbf{S E})$ & p-value & $\beta(\mathrm{SE})$ & p-value & p-value & $\beta(\mathbf{S E})$ & p-value \\
\hline Pre MBSR daytime BP* & $0.80(0.13)$ & $<.0001$ & $0.90(0.11)$ & $<.0001$ & $0.92(0.16)$ & $<.0001$ & $0.85(0.14)$ & $<.0001$ & & $1.09(0.25)$ & 0.001 \\
\hline Pre MBSR AXI* & $0.19(0.23)$ & 0.43 & $0.03(0.16)$ & 0.85 & $0.12(0.27)$ & 0.66 & $-0.08(0.21)$ & 0.73 & & $0.35(0.38)$ & 0.37 \\
\hline Post MBSR AXI & $0.12(0.26)$ & 0.63 & $0.11(0.18)$ & 0.54 & $0.29(0.31)$ & 0.36 & $0.33(0.25)$ & 0.19 & & $-0.36(0.38)$ & 0.36 \\
\hline Age & $-0.08(0.09)$ & 0.35 & $-0.13(0.06)$ & 0.03 & $-0.18(0.10)$ & 0.08 & $-0.16(0.08)$ & 0.052 & & $-0.05(0.12)$ & 0.70 \\
\hline BMI* & $0.06(0.18)$ & 0.76 & $-0.10(0.13)$ & 0.44 & $-0.04(0.17)$ & 0.82 & $-0.11(0.13)$ & 0.43 & & $0.34(0.45)$ & 0.47 \\
\hline Sex (female) & $-4.29(2.01)$ & 0.04 & $-1.05(1.40)$ & 0.45 & & & & & & & \\
\hline FHx premature CVD (Yes) & $-3.27(2.07)$ & 0.12 & $-3.21(1.44)$ & 0.03 & $-4.64(2.35)$ & 0.06 & $-3.61(1.83)$ & 0.06 & & $-2.65(3.22)$ & 0.43 \\
\hline Drink $\geq 10$ drinks/wk (Yes)* & $2.39(2.34)$ & 0.31 & $2.81(1.63)$ & 0.09 & $1.89(2.72)$ & 0.49 & $3.72(2.16)$ & 0.10 & & $-0.80(3.95)$ & 0.84 \\
\hline Relaxation regularly (Yes)* & $2.77(2.91)$ & 0.35 & $0.60(2.03)$ & 0.77 & $3.49(2.51)$ & 0.18 & $0.51(2.00)$ & 0.80 & & 0.00 & \\
\hline Race (Causasian) & $-1.71(2.70)$ & 0.53 & $-2.92(1.89)$ & 0.13 & $0.36(2.62)$ & 0.89 & $-2.81(2.07)$ & 0.19 & & $-4.33(5.69)$ & 0.46 \\
\hline Education (> high school) & $1.79(2.67)$ & 0.51 & $0.98(1.86)$ & 0.60 & $1.93(2.53)$ & 0.45 & $1.16(2.00)$ & 0.57 & & $1.21(5.33)$ & 0.83 \\
\hline Regular Exercise (Yes)* & $-5.07(3.02)$ & 0.10 & $-3.22(2.10)$ & 0.13 & $-1.00(3.08)$ & 0.75 & $-1.46(2.45)$ & 0.56 & & $-9.15(5.69)$ & 0.13 \\
\hline
\end{tabular}

* Baseline covariates. NOTE1: Grey fill indicates an overall null model. NOTE2: only 1 male subject regularly practiced relaxation, thus, that $\beta$ coefficient is zero for males. Family history (FHx), cardiovascular disease (CVD). 
Table 38. Effects of post intervention anger expressed outward on post intervention 24-hour blood pressure after adjustment for baseline blood pressure, baseline psychosocial functioning and other covariates

\begin{tabular}{|c|c|c|c|c|c|c|c|c|c|c|c|}
\hline \multirow[b]{3}{*}{ Predictors } & \multicolumn{4}{|l|}{ All Subjects } & \multicolumn{4}{|l|}{ Females } & \multicolumn{3}{|l|}{ Males } \\
\hline & Systolic & & Diastolic & & Systolic & & Diastolic & & Systolic & Diastolic & \\
\hline & $\beta$ (SE) & p-value & $\beta$ (SE) & p-value & $\beta$ (SE) & p-value & $\beta$ (SE) & p-value & p-value & $\beta$ (SE) & p-value \\
\hline Pre MBSR 24-hour BP** & $0.85(0.14)$ & $<.0001$ & $1.02(0.12)$ & $<.0001$ & $0.95(0.18)$ & $<.0001$ & $0.93(0.16)$ & $<.0001$ & & $1.28(0.29)$ & 0.001 \\
\hline Pre MBSR AXO** & $-0.11(0.33)$ & 0.74 & $-0.03(0.21)$ & 0.88 & $-0.24(0.34)$ & 0.49 & $-0.14(0.27)$ & 0.59 & & $0.19(0.47)$ & 0.70 \\
\hline Post MBSR AXO & $0.23(0.40)$ & 0.57 & $0.00(0.27)$ & 0.99 & $0.41(0.44)$ & 0.36 & $-0.18(0.34)$ & 0.59 & & $0.09(0.57)$ & 0.88 \\
\hline Age & $-0.06(0.09)$ & 0.50 & $-0.11(0.06)$ & 0.06 & $-0.17(0.10)$ & 0.11 & $-0.16(0.07)$ & 0.04 & & $-0.06(0.12)$ & 0.64 \\
\hline BMI* & $0.06(0.19)$ & 0.74 & $-0.14(0.12)$ & 0.27 & $-0.09(0.18)$ & 0.63 & $-0.17(0.13)$ & 0.21 & & $0.15(0.43)$ & 0.73 \\
\hline Sex (female) & $-2.48(1.97)$ & 0.21 & $-0.25(1.34)$ & 0.85 & & & & & & & \\
\hline FHx premature CVD (Yes) & $-3.07(1.98)$ & 0.13 & $-3.41(1.34)$ & 0.01 & $-2.97(2.19)$ & 0.19 & $-2.90(1.66)$ & 0.09 & & $-4.03(3.37)$ & 0.25 \\
\hline Drink $\geq 10$ drinks/wk (Yes)* & $3.51(2.27)$ & 0.13 & $3.10(1.54)$ & 0.0495 & $4.89(2.78)$ & 0.09 & $5.41(2.14)$ & 0.02 & & $0.13(3.02)$ & 0.97 \\
\hline Relaxation regularly (Yes)* & $-0.79(2.74)$ & 0.78 & $-1.40(1.85)$ & 0.45 & $-1.03(2.39)$ & 0.67 & $-2.43(1.86)$ & 0.20 & & 0.00 & \\
\hline Race (Causasian) & $-1.35(2.64)$ & 0.61 & $-3.01(1.79)$ & 0.10 & $1.14(2.57)$ & 0.66 & $-2.97(2.01)$ & 0.15 & & $-1.71(4.90)$ & 0.73 \\
\hline Education (> high school) & $3.09(2.59)$ & 0.24 & $0.96(1.73)$ & 0.58 & $2.51(2.47)$ & 0.32 & $1.25(1.87)$ & 0.51 & & $3.36(5.49)$ & 0.55 \\
\hline Regular Exercise (Yes)* & $-5.69(3.02)$ & 0.07 & $-2.57(2.04)$ & 0.21 & $-2.87(3.25)$ & 0.39 & $-0.57(2.50)$ & 0.82 & & $-7.37(5.30)$ & 0.19 \\
\hline
\end{tabular}

* Baseline covariates. NOTE1: Grey fill indicates an overall null model. NOTE2: only 1 male subject regularly practiced relaxation, thus, that $\beta$ coefficient is zero for males. Family history (FHx), cardiovascular disease (CVD).

Table 39. Effects of post intervention anger expressed outward on post intervention daytime blood pressure after adjustment for baseline blood pressure, baseline psychosocial functioning and other covariates

\begin{tabular}{|c|c|c|c|c|c|c|c|c|c|c|c|}
\hline \multirow[b]{3}{*}{ Predictors } & \multicolumn{4}{|l|}{ All Subjects } & \multicolumn{4}{|l|}{ Females } & \multicolumn{3}{|l|}{ Males } \\
\hline & Systolic & & Diastolic & & Systolic & & Diastolic & & Systolic & & Diastolic \\
\hline & $\beta(\mathbf{S E})$ & p-value & $\beta(\mathbf{S E})$ & p-value & $\beta(\mathbf{S E})$ & p-value & $\beta(\mathbf{S E})$ & p-value & $\beta$ (SE) & p-value & p-value \\
\hline Pre MBSR daytime BP* & $0.77(0.14)$ & $<.0001$ & $0.90(0.11)$ & $<.0001$ & $0.91(0.16)$ & $<.0001$ & $0.81(0.14)$ & $<.0001$ & & & \\
\hline Pre MBSR AXO** & $-0.11(0.35)$ & 0.75 & $-0.03(0.23)$ & 0.90 & $-0.26(0.37)$ & 0.48 & $-0.20(0.28)$ & 0.49 & & & \\
\hline Post MBSR AXO & $0.06(0.42)$ & 0.89 & $-0.05(0.28)$ & 0.86 & $0.36(0.47)$ & 0.45 & $-0.24(0.36)$ & 0.51 & & & \\
\hline Age & $-0.08(0.09)$ & 0.35 & $-0.14(0.06)$ & 0.02 & $-0.19(0.11)$ & 0.08 & $-0.20(0.08)$ & 0.01 & & & \\
\hline BMI* & $0.08(0.19)$ & 0.68 & $-0.10(0.13)$ & 0.45 & $-0.02(0.18)$ & 0.91 & $-0.12(0.14)$ & 0.39 & & & \\
\hline Sex (female) & $-4.48(2.06)$ & 0.03 & $-1.22(1.43)$ & 0.40 & & & & & & & \\
\hline FHx premature CVD (Yes) & $-2.72(2.07)$ & 0.20 & $-3.10(1.43)$ & 0.03 & $-3.69(2.34)$ & 0.13 & $-3.44(1.77)$ & 0.06 & & & \\
\hline Drink $\geq 10$ drinks/wk (Yes)* & $3.05(2.39)$ & 0.21 & $2.96(1.64)$ & 0.08 & $2.93(3.00)$ & 0.34 & $4.27(2.29)$ & 0.07 & & & \\
\hline Relaxation regularly (Yes)* & $1.46(2.87)$ & 0.61 & $0.13(1.99)$ & 0.95 & $2.10(2.56)$ & 0.42 & $-0.62(1.96)$ & 0.76 & & & \\
\hline Race (Causasian) & $-2.22(2.76)$ & 0.43 & $-3.15(1.92)$ & 0.11 & $0.33(2.73)$ & 0.90 & $-2.89(2.11)$ & 0.18 & & & \\
\hline Education (> high school) & $2.04(2.70)$ & 0.45 & $1.07(1.85)$ & 0.57 & $2.10(2.61)$ & 0.43 & $1.60(2.00)$ & 0.43 & & & \\
\hline Regular Exercise (Yes)* & $-5.28(3.17)$ & 0.10 & $-3.17(2.18)$ & 0.15 & $-2.41(3.47)$ & 0.49 & $-0.57(2.67)$ & 0.83 & & & \\
\hline
\end{tabular}

* Baseline covariates. NOTE1: Grey fill indicates an overall null model. Family history (FHx), cardiovascular disease (CVD). 
Table 40. Effects of post intervention perceived stress on post intervention 24-hour blood pressure after adjustment for baseline blood pressure, baseline psychosocial functioning and other covariates

\begin{tabular}{|c|c|c|c|c|c|c|c|c|c|c|c|c|}
\hline \multirow[b]{3}{*}{ Predictors } & \multicolumn{4}{|l|}{ All Subjects } & \multicolumn{4}{|l|}{ Females } & \multicolumn{4}{|l|}{ Males } \\
\hline & Systolic & & Diastolic & & Systolic & & Diastolic & & Systolic & & Diastolic & \\
\hline & $\beta(\mathbf{S E})$ & p-value & $\beta(\mathbf{S E})$ & p-value & $\beta(\mathrm{SE})$ & p-value & $\beta(\mathbf{S E})$ & p-value & $\beta(\mathrm{SE})$ & p-value & $\beta(\mathrm{SE})$ & p-value \\
\hline Pre MBSR 24-hour BP** & $0.85(0.11)$ & $<.0001$ & $1.00(0.10)$ & $<.0001$ & $0.81(0.12)$ & $<.0001$ & $0.94(0.12)$ & $<.0001$ & $0.40(0.26)$ & 0.13 & $1.17(0.23)$ & 0.0001 \\
\hline Pre MBSR PS* & $0.11(0.12)$ & 0.36 & $0.07(0.08)$ & 0.40 & $0.32(0.12)$ & 0.01 & $0.14(0.09)$ & 0.15 & $-0.05(0.22)$ & 0.81 & $0.14(0.17)$ & 0.43 \\
\hline Post MBSR PS & $-0.03(0.16)$ & 0.84 & $-0.06(0.11)$ & 0.60 & $-0.35(0.15)$ & 0.03 & $-0.25(0.13)$ & 0.07 & $0.83(0.32)$ & 0.02 & $0.18(0.20)$ & 0.38 \\
\hline Age & $-0.09(0.08)$ & 0.29 & $-0.12(0.05)$ & 0.02 & $-0.22(0.09)$ & 0.02 & $-0.19(0.07)$ & 0.01 & $0.26(0.13)$ & 0.07 & $0.04(0.10)$ & 0.73 \\
\hline BMI* & $0.05(0.17)$ & 0.76 & $-0.16(0.12)$ & 0.16 & $-0.08(0.15)$ & 0.62 & $-0.19(0.12)$ & 0.12 & $0.18(0.46)$ & 0.70 & $0.05(0.32)$ & 0.88 \\
\hline Sex (female) & $-3.44(1.70)$ & 0.048 & $-1.12(1.16)$ & 0.34 & & & & & & & & \\
\hline FHx premature CVD (Yes) & $-3.16(1.89)$ & 0.10 & $-3.63(1.30)$ & 0.01 & $-2.50(2.03)$ & 0.23 & $-2.86(1.66)$ & 0.10 & $-6.01(3.34)$ & 0.09 & $-5.65(2.61)$ & 0.047 \\
\hline Drink $\geq 10$ drinks/wk (Yes)* & $3.29(2.15)$ & 0.13 & $2.86(1.47)$ & 0.06 & $3.37(2.44)$ & 0.18 & $5.06(2.00)$ & 0.02 & $3.76(3.60)$ & 0.31 & $0.87(2.58)$ & 0.74 \\
\hline Relaxation regularly (Yes)* & $0.43(2.55)$ & 0.87 & $-0.47(1.74)$ & 0.79 & $-0.90(2.15)$ & 0.68 & $-1.91(1.76)$ & 0.29 & 0.00 & & 0.00 & \\
\hline Race (Causasian) & $-0.85(2.35)$ & 0.72 & $-2.73(1.61)$ & 0.10 & $1.10(2.20)$ & 0.62 & $-3.24(1.81)$ & 0.08 & $-5.77(4.37)$ & 0.21 & $-2.01(3.65)$ & 0.59 \\
\hline Education (> high school) & $-0.31(2.31)$ & 0.89 & $-0.81(1.57)$ & 0.61 & $-0.07(2.22)$ & 0.97 & $-0.10(1.80)$ & 0.96 & $3.23(5.83)$ & 0.59 & $-0.55(3.88)$ & 0.89 \\
\hline Regular Exercise (Yes)* & $-3.73(2.80)$ & 0.19 & $-1.99(1.92)$ & 0.30 & $-0.49(2.80)$ & 0.86 & $-1.38(2.22)$ & 0.54 & $-17.19(7.16)$ & 0.03 & $-4.98(4.11)$ & 0.24 \\
\hline
\end{tabular}

* Baseline covariates. NOTE1: only 1 male subject regularly practiced relaxation, thus, that $\beta$ coefficient is zero for males. Family history (FHx), cardiovascular disease (CVD).

Table 41. Effects of post intervention perceived stress on post intervention daytime blood pressure after adjustment for baseline blood pressure, baseline psychosocial functioning and other covariates

\begin{tabular}{|c|c|c|c|c|c|c|c|c|c|c|c|c|}
\hline \multirow[b]{3}{*}{ Predictors } & \multicolumn{4}{|l|}{ All Subjects } & \multicolumn{4}{|l|}{ Females } & \multicolumn{4}{|l|}{ Males } \\
\hline & Systolic & & Diastolic & & Systolic & & Diastolic & & Systolic & & Diastolic & \\
\hline & $\beta(\mathrm{SE})$ & p-value & $\beta(\mathbf{S E})$ & p-value & $\beta(\mathrm{SE})$ & p-value & $\beta(\mathbf{S E})$ & p-value & $\beta(\mathrm{SE})$ & p-value & $\beta(\mathbf{S E})$ & p-value \\
\hline Pre MBSR daytime BP* & $0.86(0.11)$ & $<.0001$ & $0.96(0.10)$ & $<.0001$ & $0.89(0.13)$ & $<.0001$ & $0.95(0.12)$ & $<.0001$ & $0.30(0.28)$ & 0.30 & $1.08(0.23)$ & 0.0003 \\
\hline Pre MBSR PS* & $0.16(0.12)$ & 0.19 & $0.10(0.09)$ & 0.26 & $0.32(0.12)$ & 0.02 & $0.12(0.10)$ & 0.23 & $-0.05(0.27)$ & 0.86 & $0.29(0.20)$ & 0.16 \\
\hline Post MBSR PS & $-0.05(0.17)$ & 0.74 & $-0.10(0.12)$ & 0.38 & $-0.33(0.17)$ & 0.055 & $-0.27(0.14)$ & 0.063 & $0.91(0.36)$ & 0.02 & $0.15(0.23)$ & 0.52 \\
\hline Age & $-0.11(0.09)$ & 0.22 & $-0.14(0.06)$ & 0.01 & $-0.27(0.09)$ & 0.01 & $-0.22(0.07)$ & 0.01 & $0.27(0.15)$ & 0.10 & $0.05(0.11)$ & 0.67 \\
\hline BMI* & $0.07(0.18)$ & 0.71 & $-0.09(0.12)$ & 0.45 & $-0.08(0.16)$ & 0.61 & $-0.14(0.13)$ & 0.29 & $0.16(0.51)$ & 0.76 & $0.21(0.36)$ & 0.56 \\
\hline Sex (female) & $-5.27(1.81)$ & 0.01 & $-1.97(1.24)$ & 0.12 & & & & & & & & \\
\hline FHx premature CVD (Yes) & $-3.17(2.02)$ & 0.12 & $-3.50(1.38)$ & 0.01 & $-3.63(2.19)$ & 0.11 & $-3.42(1.76)$ & 0.06 & $-5.02(3.72)$ & 0.20 & $-5.06(2.95)$ & 0.11 \\
\hline Drink $\geq 10$ drinks/wk (Yes)* & $2.33(2.29)$ & 0.31 & $2.31(1.56)$ & 0.15 & $1.08(2.61)$ & 0.68 & $3.62(2.11)$ & 0.10 & $3.35(4.02)$ & 0.42 & $0.12(2.90)$ & 0.97 \\
\hline Relaxation regularly (Yes)* & $3.37(2.71)$ & 0.22 & $1.08(1.85)$ & 0.56 & $2.70(2.30)$ & 0.25 & $-0.03(1.86)$ & 0.99 & 0.00 & & 0.00 & \\
\hline Race (Causasian) & $-1.42(2.50)$ & 0.57 & $-2.79(1.72)$ & 0.11 & $0.88(2.38)$ & 0.71 & $-3.30(1.92)$ & 0.10 & $-6.27(4.94)$ & 0.22 & $-2.68(4.03)$ & 0.52 \\
\hline Education (> high school) & $-0.35(2.46)$ & 0.89 & $-0.33(1.67)$ & 0.84 & $-0.22(2.39)$ & 0.93 & $0.16(1.91)$ & 0.93 & $4.01(6.19)$ & 0.53 & $2.32(4.43)$ & 0.61 \\
\hline Regular Exercise (Yes)* & $-3.58(2.98)$ & 0.23 & $-2.69(2.03)$ & 0.19 & $-0.69(2.97)$ & 0.82 & $-1.26(2.35)$ & 0.60 & $-19.30(8.13)$ & 0.03 & $-7.24(4.64)$ & 0.14 \\
\hline
\end{tabular}

* Baseline covariates. NOTE1: only 1 male subject regularly practiced relaxation, thus, that $\beta$ coefficient is zero for males. Family history

(FHx), cardiovascular disease (CVD). 
Table 42. Effects of post intervention trait anger on post intervention nighttime blood pressure after adjustment for baseline blood pressure, baseline psychosocial functioning and other covariates

\begin{tabular}{|c|c|c|c|c|c|c|c|c|c|c|c|c|}
\hline \multirow[b]{3}{*}{ Predictors } & \multicolumn{4}{|l|}{ All Subjects } & \multicolumn{4}{|l|}{ Females } & \multicolumn{4}{|l|}{ Males } \\
\hline & Systolic & & Diastolic & & Systolic & & Diastolic & & Systolic & & Diastolic & \\
\hline & $\beta$ (SE) & p-value & $\beta$ (SE) & p-value & $\beta$ (SE) & p-value & $\beta$ (SE) & p-value & $\beta(\mathbf{S E})$ & p-value & $\beta$ (SE) & p-value \\
\hline Pre MBSR nighttime BP* & $0.85(0.12)$ & $<.0001$ & $0.83(0.09)$ & $<.0001$ & $0.95(0.17)$ & $<.0001$ & $0.81(0.13)$ & $<.0001$ & $0.84(0.21)$ & 0.002 & $0.89(0.18)$ & 0.0003 \\
\hline Pre MBSR T-Ang* & $-0.02(0.30)$ & 0.94 & $-0.15(0.19)$ & 0.44 & $0.20(0.42)$ & 0.64 & $-0.24(0.28)$ & 0.39 & $-0.42(0.72)$ & 0.57 & $-0.35(0.49)$ & 0.48 \\
\hline Post MBSR T-Ang & $0.14(0.44)$ & 0.76 & $0.35(0.28)$ & 0.22 & $-0.37(0.61)$ & 0.56 & $0.25(0.41)$ & 0.55 & $0.92(1.17)$ & 0.44 & $1.00(0.81)$ & 0.24 \\
\hline Age & $0.02(0.09)$ & 0.83 & $-0.03(0.06)$ & 0.61 & $-0.18(0.13)$ & 0.17 & $-0.10(0.08)$ & 0.23 & $0.29(0.15)$ & 0.07 & $0.08(0.11)$ & 0.46 \\
\hline BMI* & $0.06(0.20)$ & 0.77 & $-0.13(0.12)$ & 0.29 & $-0.24(0.22)$ & 0.28 & $-0.19(0.14)$ & 0.18 & $1.07(0.66)$ & 0.13 & $0.23(0.47)$ & 0.64 \\
\hline Sex (female) & $-1.56(2.13)$ & 0.47 & $-0.06(1.39)$ & 0.97 & & & & & & & & \\
\hline FHx premature CVD (Yes) & $-1.93(2.18)$ & 0.38 & $-2.47(1.40)$ & 0.08 & $-3.91(2.94)$ & 0.20 & $-2.21(1.96)$ & 0.27 & $1.35(4.01)$ & 0.74 & $-1.05(2.96)$ & 0.73 \\
\hline Drink $\geq 10$ drinks/wk (Yes)* & $4.58(2.52)$ & 0.08 & $3.00(1.65)$ & 0.08 & $3.51(3.54)$ & 0.33 & $5.99(2.43)$ & 0.02 & $-0.57(3.99)$ & 0.89 & $-1.39(2.86)$ & 0.64 \\
\hline Relaxation regularly (Yes)* & $-3.97(3.02)$ & 0.19 & $-3.83(1.94)$ & 0.054 & $-3.98(3.08)$ & 0.21 & $-4.55(2.11)$ & 0.04 & 0.00 & & 0.00 & \\
\hline Race (Causasian) & $1.83(2.91)$ & 0.53 & $-2.81(1.85)$ & 0.14 & $3.78(3.44)$ & 0.28 & $-2.45(2.31)$ & 0.30 & $-2.51(5.42)$ & 0.65 & $-3.56(4.15)$ & 0.41 \\
\hline Education (> high school) & $3.30(2.85)$ & 0.25 & $0.80(1.81)$ & 0.66 & $1.67(3.37)$ & 0.62 & $-0.17(2.23)$ & 0.94 & $13.94(10.18)$ & 0.20 & $8.46(7.43)$ & 0.28 \\
\hline Regular Exercise (Yes)* & $-6.10(3.23)$ & 0.07 & $-0.65(2.07)$ & 0.76 & $-1.90(3.86)$ & 0.63 & $-1.89(2.49)$ & 0.45 & $-13.52(7.82)$ & 0.11 & $0.53(5.21)$ & 0.92 \\
\hline
\end{tabular}

* Baseline covariates. NOTE1: only 1 male subject regularly practiced relaxation, thus, that $\beta$ coefficient is zero for males. Family history (FHx), cardiovascular disease (CVD).

Table 43. Effects of post intervention trait anger temperament on post intervention nighttime blood pressure after adjustment for baseline blood pressure, baseline psychosocial functioning and other covariates

\begin{tabular}{|c|c|c|c|c|c|c|c|c|c|c|c|c|}
\hline \multirow[b]{3}{*}{ Predictors } & \multicolumn{4}{|l|}{ All Subjects } & \multicolumn{4}{|l|}{ Females } & \multicolumn{4}{|l|}{ Males } \\
\hline & Systolic & & Diastolic & & Systolic & & Diastolic & & Systolic & & Diastolic & \\
\hline & $\beta(\mathbf{S E})$ & p-value & $\beta(\mathbf{S E})$ & p-value & $\beta(\mathbf{S E})$ & p-value & $\beta(\mathbf{S E})$ & p-value & $\beta(\mathbf{S E})$ & p-value & $\beta(\mathbf{S E})$ & p-value \\
\hline Pre MBSR nighttime BP* & $0.85(0.12)$ & $<.0001$ & $0.82(0.10)$ & $<.0001$ & $0.96(0.17)$ & $<.0001$ & $0.77(0.13)$ & $<.0001$ & $0.85(0.21)$ & 0.002 & $0.82(0.18)$ & 0.001 \\
\hline Pre MBSR T-Ang/T** & $-0.26(0.62)$ & 0.67 & $-0.31(0.40)$ & 0.44 & $-0.90(0.86)$ & 0.31 & $-0.71(0.60)$ & 0.24 & $-1.10(1.30)$ & 0.41 & $-0.61(0.92)$ & 0.52 \\
\hline Post MBSR T-Ang/T & $0.81(0.91)$ & 0.37 & $0.45(0.60)$ & 0.46 & $1.95(1.30)$ & 0.15 & $1.02(0.92)$ & 0.28 & $1.94(2.14)$ & 0.38 & $1.40(1.56)$ & 0.39 \\
\hline Age & $0.01(0.09)$ & 0.87 & $-0.05(0.06)$ & 0.43 & $-0.21(0.12)$ & 0.11 & $-0.12(0.08)$ & 0.15 & $0.30(0.15)$ & 0.06 & $0.10(0.12)$ & 0.42 \\
\hline BMI* & $0.06(0.20)$ & 0.75 & $-0.13(0.13)$ & 0.32 & $-0.29(0.21)$ & 0.18 & $-0.20(0.13)$ & 0.15 & $1.34(0.56)$ & 0.03 & $0.50(0.41)$ & 0.24 \\
\hline Sex (female) & $-1.19(2.16)$ & 0.58 & $0.10(1.42)$ & 0.95 & & & & & & & & \\
\hline FHx premature CVD (Yes) & $-1.90(2.15)$ & 0.38 & $-2.53(1.41)$ & 0.08 & $-2.29(2.79)$ & 0.42 & 1.89) & 0.28 & $2.25(3.88)$ & 0.57 & $0.29(2.94)$ & 0.92 \\
\hline Drink $\geq 10$ drinks/wk (Yes)* & $4.46(2.54)$ & 0.09 & $3.12(1.69)$ & 0.07 & $4.38(3.36)$ & 0.20 & $5.76(2.34)$ & 0.02 & $-1.13(4.22)$ & 0.79 & $-2.12(3.21)$ & 0.52 \\
\hline Relaxation regularly (Yes)* & $-4.18(3.04)$ & 0.18 & $-4.27(1.98)$ & 0.04 & $-4.93(3.07)$ & 0.12 & $-4.80(2.12)$ & 0.03 & 0.00 & & 0.00 & \\
\hline Race (Causasian) & $1.57(2.90)$ & 0.59 & $-2.73(1.88)$ & 0.15 & $2.37(3.25)$ & 0.47 & $-2.34(2.23)$ & 0.30 & $-3.95(5.26)$ & 0.47 & $-5.26(4.22)$ & 0.24 \\
\hline Education (> high school) & $3.23(2.85)$ & 0.26 & $0.54(1.85)$ & 0.77 & $-1.71(3.64)$ & 0.64 & $-1.25(2.46)$ & 0.61 & $12.73(9.19)$ & 0.19 & $4.94(6.94)$ & 0.49 \\
\hline Regular Exercise (Yes)* & $-5.97(3.20)$ & 0.07 & $-0.73(2.09)$ & 0.73 & $-3.09(3.73)$ & 0.41 & $-1.96(2.46)$ & 0.43 & $-12.02(7.45)$ & 0.13 & $2.38(5.05)$ & 0.65 \\
\hline
\end{tabular}

* Baseline covariates. NOTE1: only 1 male subject regularly practiced relaxation, thus, that $\beta$ coefficient is zero for males. Family history

(FHx), cardiovascular disease (CVD). 
Table 44. Effects of post intervention trait anger temperament as a multilevel categorical predictor variable divided into quartiles on post intervention nighttime blood pressure after adjustment for baseline blood pressure, baseline psychosocial functioning and other covariates

\begin{tabular}{|c|c|c|c|c|c|c|c|c|c|c|c|}
\hline \multirow[b]{3}{*}{ Predictors } & \multicolumn{4}{|l|}{ All Subjects } & \multicolumn{4}{|l|}{ Females } & \multicolumn{3}{|l|}{ Males } \\
\hline & \multirow{2}{*}{$\begin{array}{l}\text { Systolic } \\
\beta \text { (SE) }\end{array}$} & \multicolumn{2}{|r|}{ Diastolic } & \multicolumn{2}{|r|}{ Systolic } & \multicolumn{2}{|r|}{ Diastolic } & \multicolumn{2}{|r|}{ Systolic } & \multicolumn{2}{|r|}{ Diastolic } \\
\hline & & p-value & $\beta(\mathbf{S E})$ & p-value & $\beta(\mathrm{SE})$ & p-value & $\beta(\mathrm{SE})$ & p-value & $\beta(\mathrm{SE})$ & p-value & p-value \\
\hline Pre MBSR nighttime BP* & $0.83(0.12)$ & $<.0001$ & $0.83(0.10)$ & $<.0001$ & $0.89(0.18)$ & $<.0001$ & $0.80(0.14)$ & $<.0001$ & $0.74(0.23)$ & 0.01 & \\
\hline Pre MBSR T-Ang/T* & $-0.54(0.63)$ & 0.40 & $-0.24(0.42)$ & 0.57 & $-0.62(0.86)$ & 0.48 & $-0.31(0.60)$ & 0.61 & $-0.53(1.39)$ & 0.71 & \\
\hline Post MBSR T-Ang/T & & & & & & & & & & & \\
\hline Q1 vs Q4 & $-4.69(4.10)$ & 0.26 & $-1.49(2.76)$ & 0.59 & $-5.29(5.33)$ & 0.33 & $-1.11(3.75)$ & 0.77 & $-6.29(11.34)$ & 0.59 & \\
\hline Q2 vs Q4 & $-7.50(4.37)$ & 0.09 & $-1.46(2.94)$ & 0.62 & $-8.61(5.95)$ & 0.16 & $-1.61(4.13)$ & 0.70 & $-4.26(10.37)$ & 0.69 & \\
\hline Q3 vs Q4 & $-4.00(3.54)$ & 0.26 & $-1.51(2.38)$ & 0.53 & $-4.73(4.24)$ & 0.28 & $-0.78(2.96)$ & 0.80 & $-11.62(10.24)$ & 0.28 & \\
\hline Age & $0.01(0.09)$ & 0.92 & $-0.05(0.06)$ & 0.42 & $-0.17(0.13)$ & 0.20 & $-0.10(0.08)$ & 0.24 & $0.32(0.16)$ & 0.07 & \\
\hline BMI* & $0.05(0.20)$ & 0.79 & $-0.13(0.13)$ & 0.30 & $-0.23(0.22)$ & 0.31 & $-0.18(0.15)$ & 0.23 & $1.65(0.66)$ & 0.03 & \\
\hline Sex (female) & $-0.88(2.16)$ & 0.69 & $0.11(1.46)$ & 0.94 & & & & & & & \\
\hline FHx premature CVD (Yes) & $-2.16(2.17)$ & 0.32 & $-2.68(1.46)$ & 0.07 & $-3.33(2.90)$ & 0.26 & $-2.59(2.02)$ & 0.21 & $0.75(4.31)$ & 0.87 & \\
\hline Drink $\geq 10$ drinks/wk $(Y e s)^{*}$ & $5.36(2.79)$ & 0.06 & $3.04(1.89)$ & 0.12 & $5.34(3.99)$ & 0.19 & $5.96(2.84)$ & 0.046 & $-4.99(5.98)$ & 0.42 & \\
\hline Relaxation regularly (Yes)* & $-4.93(3.15)$ & 0.12 & $-4.17(2.10)$ & 0.054 & $-4.79(3.28)$ & 0.16 & $-4.62(2.35)$ & 0.06 & 0.00 & & \\
\hline Race (Causasian) & $0.38(3.12)$ & 0.90 & $-2.59(2.08)$ & 0.22 & $2.19(3.58)$ & 0.55 & $-2.29(2.53)$ & 0.37 & $-0.90(6.53)$ & 0.89 & \\
\hline Education (> high school) & $3.10(2.90)$ & 0.29 & $0.61(1.94)$ & 0.75 & $0.09(3.53)$ & 0.98 & $-0.03(2.43)$ & 0.99 & $12.02(8.75)$ & 0.20 & \\
\hline Regular Exercise (Yes)* & $-6.93(3.25)$ & 0.04 & $-0.82(2.18)$ & 0.71 & $-4.18(4.06)$ & 0.31 & $-2.01(2.79)$ & 0.48 & $-11.66(7.90)$ & 0.17 & \\
\hline
\end{tabular}

* Baseline covariates. NOTE1: Grey fill indicate s an overall null model. NOTE2: only 1 male subject regularly practiced relaxation, thus, that $\beta$ coefficient is zero for males. Family history (FHx), cardiovascular disease (CVD). Q1= bottom $25^{\text {th }}$ percent quartile, Q2=second quartile, $\mathrm{Q} 3=$ third quartile, $\mathrm{Q} 4=$ top $25^{\text {th }}$ percent quartile. 
Table 45. Effects of post intervention trait anger reaction on post intervention nighttime blood pressure after adjustment for baseline blood pressure, baseline psychosocial functioning and other covariates

\begin{tabular}{|c|c|c|c|c|c|c|c|c|c|c|c|c|}
\hline \multirow[b]{3}{*}{ Predictors } & \multicolumn{4}{|l|}{ All Subjects } & \multicolumn{4}{|l|}{ Females } & \multicolumn{4}{|l|}{ Males } \\
\hline & Systolic & & Diastolic & & Systolic & & Diastolic & & Systolic & & Diastolic & \\
\hline & $\beta(\mathbf{S E})$ & p-value & $\beta(\mathbf{S E})$ & p-value & $\beta$ (SE) & p-value & $\beta$ (SE) & p-value & $\beta$ (SE) & p-value & $\beta$ (SE) & p-value \\
\hline Pre MBSR nighttime $\mathrm{BP}^{*}$ & $0.85(0.12)$ & $<.0001$ & $0.84(0.09)$ & $<.0001$ & $0.95(0.17)$ & $<.0001$ & $0.85(0.13)$ & $<.0001$ & $0.80(0.20)$ & 0.002 & $0.86(0.18)$ & 0.0004 \\
\hline Pre MBSR T-Ang/R* & $-0.03(0.56)$ & 0.96 & $-0.22(0.35)$ & 0.54 & $0.49(0.64)$ & 0.45 & $-0.39(0.44)$ & 0.38 & $-0.10(1.38)$ & 0.95 & $0.14(0.97)$ & 0.89 \\
\hline Post MBSR T-Ang/R & $0.05(0.67)$ & 0.94 & $0.60(0.42)$ & 0.16 & $-1.10(0.79)$ & 0.17 & $0.20(0.54)$ & 0.72 & $0.59(1.73)$ & 0.74 & $0.63(1.23)$ & 0.62 \\
\hline Age & $0.01(0.09)$ & 0.91 & $-0.03(0.06)$ & 0.65 & $-0.23(0.12)$ & 0.08 & $-0.10(0.08)$ & 0.23 & $0.28(0.15)$ & 0.08 & $0.07(0.11)$ & 0.52 \\
\hline BMI* & $0.02(0.22)$ & 0.93 & $-0.16(0.14)$ & 0.24 & $-0.38(0.24)$ & 0.12 & $-0.25(0.16)$ & 0.13 & $1.22(0.75)$ & 0.13 & $0.44(0.52)$ & 0.41 \\
\hline Sex (female) & $-1.67(2.18)$ & 0.45 & $-0.39(1.40)$ & 0.78 & & & & & & & & \\
\hline FHx premature CVD (Yes) & $-1.83(2.21)$ & 0.41 & $-2.49(1.40)$ & 0.08 & $-3.33(2.79)$ & 0.24 & $-2.13(1.94)$ & 0.28 & $1.42(4.24)$ & 0.74 & $-0.84(3.04)$ & 0.79 \\
\hline Drink $\geq 10$ drinks/wk (Yes)* & $4.75(2.52)$ & 0.07 & $3.17(1.64)$ & 0.06 & $3.98(3.40)$ & 0.25 & $6.32(2.43)$ & 0.02 & $-0.04(4.11)$ & 0.99 & $-0.70(2.94)$ & 0.81 \\
\hline Relaxation regularly (Yes)* & $-4.10(3.04)$ & 0.18 & $-3.55(1.93)$ & 0.07 & $-4.92(2.92)$ & 0.10 & $-4.66(2.08)$ & 0.03 & 0.00 & & 0.00 & \\
\hline Race (Causasian) & $1.98(2.90)$ & 0.50 & $-2.65(1.83)$ & 0.15 & $4.04(3.22)$ & 0.22 & $-2.31(2.26)$ & 0.32 & $-2.68(6.14)$ & 0.67 & $-4.09(4.65)$ & 0.40 \\
\hline Education (> high school) & $3.00(2.95)$ & 0.31 & $0.68(1.85)$ & 0.72 & $0.52(3.24)$ & 0.87 & $-0.05(2.24)$ & 0.98 & $9.72(8.56)$ & 0.28 & $3.51(6.13)$ & 0.58 \\
\hline Regular Exercise (Yes)* & $-6.25(3.27)$ & 0.06 & $-0.58(2.07)$ & 0.78 & $-2.36(3.71)$ & 0.53 & $-2.14(2.49)$ & 0.40 & $-12.49(8.10)$ & 0.15 & $2.29(5.05)$ & 0.66 \\
\hline
\end{tabular}

* Baseline covariates. NOTE1: only 1 male subject regularly practiced relaxation, thus, that $\beta$ coefficient is zero for males. Family history

(FHx), cardiovascular disease (CVD).

Table 46. Effects of post intervention anger expressed inward on post intervention nighttime blood pressure after adjustment for baseline blood pressure, baseline psychosocial functioning and other covariates

\begin{tabular}{|c|c|c|c|c|c|c|c|c|c|c|c|c|}
\hline \multirow[b]{3}{*}{ Predictors } & \multicolumn{4}{|l|}{ All Subjects } & \multicolumn{4}{|l|}{ Females } & \multicolumn{4}{|l|}{ Males } \\
\hline & Systolic & & Diastolic & & Systolic & & Diastolic & & Systolic & & Diastolic & \\
\hline & $\beta(\mathbf{S E})$ & p-value & $\beta(\mathbf{S E})$ & p-value & $\beta(\mathbf{S E})$ & p-value & $\beta(\mathbf{S E})$ & p-value & $\beta(\mathbf{S E})$ & p-value & $\beta(\mathbf{S E})$ & p-value \\
\hline Pre MBSR nighttime BP* & $0.83(0.12)$ & $<.0001$ & $0.82(0.10)$ & $<.0001$ & $0.85(0.21)$ & 0.0003 & $0.79(0.14)$ & $<.0001$ & $0.84(0.20)$ & 0.001 & $0.84(0.19)$ & 0.001 \\
\hline Pre MBSR AXI* & $-0.10(0.24)$ & 0.69 & $-0.12(0.16)$ & 0.48 & $0.21(0.36)$ & 0.56 & $-0.03(0.23)$ & 0.90 & $0.06(0.46)$ & 0.90 & $0.03(0.36)$ & 0.94 \\
\hline Post MBSR AXI & $0.52(0.27)$ & 0.055 & $0.28(0.18)$ & 0.12 & $0.15(0.39)$ & 0.71 & $0.14(0.26)$ & 0.60 & $0.52(0.46)$ & 0.27 & $0.36(0.34)$ & 0.32 \\
\hline Age & $0.04(0.09)$ & 0.64 & $-0.03(0.06)$ & 0.55 & $-0.13(0.13)$ & 0.32 & $-0.08(0.08)$ & 0.31 & $0.28(0.14)$ & 0.08 & $0.08(0.12)$ & 0.49 \\
\hline BMI* & $0.09(0.19)$ & 0.66 & $-0.12(0.12)$ & 0.34 & $-0.19(0.22)$ & 0.41 & $-0.17(0.14)$ & 0.24 & $1.23(0.55)$ & 0.046 & $0.42(0.41)$ & 0.33 \\
\hline Sex (female) & $-1.17(2.07)$ & 0.57 & $0.11(1.38)$ & 0.93 & & & & & & & & \\
\hline FHx premature CVD (Yes) & $-2.07(2.12)$ & 0.33 & $-2.57(1.40)$ & 0.07 & $-3.98(2.85)$ & 0.17 & $-2.82(1.97)$ & 0.16 & $1.66(3.82)$ & 0.67 & $-0.34(2.92)$ & 0.91 \\
\hline Drink $\geq 10$ drinks/wk (Yes)* & $4.38(2.44)$ & 0.08 & $2.95(1.67)$ & 0.08 & $3.39(3.42)$ & 0.33 & $5.23(2.38)$ & 0.04 & $-1.29(4.75)$ & 0.79 & $-1.69(3.81)$ & 0.66 \\
\hline Relaxation regularly (Yes)* & $-3.47(3.09)$ & 0.27 & $-3.97(2.06)$ & 0.06 & $-2.47(3.47)$ & 0.48 & $-3.91(2.32)$ & 0.10 & 0.00 & & 0.00 & \\
\hline Race (Causasian) & $2.97(2.81)$ & 0.30 & $-2.00(1.85)$ & 0.29 & $3.17(3.27)$ & 0.34 & $-1.88(2.25)$ & 0.41 & $1.18(6.63)$ & 0.86 & $-1.59(5.20)$ & 0.77 \\
\hline Education (> high school) & $2.99(2.78)$ & 0.29 & $0.75(1.81)$ & 0.68 & $0.22(3.19)$ & 0.94 & $0.34(2.09)$ & 0.87 & $8.20(6.46)$ & 0.23 & $1.14(4.94)$ & 0.82 \\
\hline Regular Exercise (Yes)* & $-5.68(3.10)$ & 0.07 & $-0.39(2.04)$ & 0.85 & $-0.92(4.05)$ & 0.82 & $-1.55(2.55)$ & 0.55 & $-11.17(7.86)$ & 0.18 & $2.73(5.71)$ & 0.61 \\
\hline
\end{tabular}

* Baseline covariates. NOTE1: only 1 male subject regularly practiced relaxation, thus, that $\beta$ coefficient is zero for males. Family history

(FHx), cardiovascular disease (CVD). 
Table 47. Effects of post intervention anger expressed outward on post intervention nighttime blood pressure after adjustment for baseline blood pressure, baseline psychosocial functioning and other covariates

\begin{tabular}{|c|c|c|c|c|c|c|c|c|c|c|c|c|}
\hline \multirow[b]{3}{*}{ Predictors } & \multicolumn{4}{|l|}{ All Subjects } & \multicolumn{4}{|l|}{ Females } & \multicolumn{4}{|l|}{ Males } \\
\hline & Systolic & & Diastolic & & Systolic & & Diastolic & & Systolic & & Diastolic & \\
\hline & $\beta$ (SE) & p-value & $\beta(\mathrm{SE})$ & p-value & $\beta$ (SE) & p-value & $\beta$ (SE) & p-value & $\beta$ (SE) & p-value & $\beta$ (SE) & p-value \\
\hline Pre MBSR nighttime BP* & $0.82(0.12)$ & $<.0001$ & $0.82(0.10)$ & $<.0001$ & $0.92(0.19)$ & $<.0001$ & $0.78(0.15)$ & $<.0001$ & $0.81(0.19)$ & 0.001 & $0.83(0.18)$ & 0.001 \\
\hline Pre MBSR AXO* & $-0.17(0.35)$ & 0.62 & $0.11(0.23)$ & 0.63 & $0.01(0.44)$ & 0.98 & $0.17(0.31)$ & 0.59 & $-0.53(0.56)$ & 0.36 & $-0.25(0.43)$ & 0.58 \\
\hline Post MBSR AXO & $0.49(0.44)$ & 0.27 & $0.03(0.28)$ & 0.93 & $0.29(0.59)$ & 0.63 & $-0.04(0.39)$ & 0.91 & $0.78(0.71)$ & 0.29 & $0.57(0.54)$ & 0.31 \\
\hline Age & $0.04(0.09)$ & 0.66 & $-0.04(0.06)$ & 0.46 & $-0.15(0.13)$ & 0.25 & $-0.08(0.08)$ & 0.28 & $0.35(0.15)$ & 0.04 & $0.13(0.12)$ & 0.30 \\
\hline BMI* & $0.10(0.20)$ & 0.62 & $-0.14(0.13)$ & 0.28 & $-0.23(0.23)$ & 0.33 & $-0.20(0.14)$ & 0.17 & $1.46(0.56)$ & 0.02 & $0.57(0.41)$ & 0.19 \\
\hline Sex (female) & $-1.15(2.15)$ & 0.60 & $-0.07(1.43)$ & 0.96 & & & & & & & & \\
\hline FHx premature CVD (Yes) & $-1.65(2.15)$ & 0.45 & $-2.65(1.42)$ & 0.07 & $-3.19(2.79)$ & 0.26 & $-2.82(1.86)$ & 0.14 & $3.66(4.01)$ & 0.38 & $1.21(3.04)$ & 0.70 \\
\hline Drink $\geq 10$ drinks/wk $(Y e s)^{*}$ & $5.00(2.49)$ & 0.0503 & $2.80(1.69)$ & 0.10 & $3.91(3.62)$ & 0.29 & $4.83(2.58)$ & 0.07 & $-0.52(3.84)$ & 0.89 & $-1.31(2.97)$ & 0.67 \\
\hline Relaxation regularly (Yes)* & $-4.04(3.03)$ & 0.19 & $-3.67(2.02)$ & 0.08 & $-3.61(3.21)$ & 0.27 & $-3.57(2.34)$ & 0.14 & 0.00 & & 0.00 & \\
\hline Race (Causasian) & $1.47(2.89)$ & 0.61 & $-2.35(1.90)$ & 0.22 & $3.31(3.38)$ & 0.34 & $-1.48(2.38)$ & 0.54 & $-4.88(5.26)$ & 0.37 & $-5.71(4.26)$ & 0.20 \\
\hline Education (> high school) & $3.15(2.82)$ & 0.27 & $0.81(1.84)$ & 0.66 & $0.63(3.18)$ & 0.84 & $0.46(2.10)$ & 0.83 & $7.91(6.98)$ & 0.28 & $1.54(5.36)$ & 0.78 \\
\hline Regular Exercise (Yes)* & $-7.09(3.29)$ & 0.04 & $-0.87(2.16)$ & 0.69 & $-3.04(4.13)$ & 0.47 & $-1.70(2.75)$ & 0.54 & $-11.43(7.41)$ & 0.15 & $2.52(4.99)$ & 0.62 \\
\hline
\end{tabular}

* Baseline covariates. NOTE1: only 1 male subject regularly practiced relaxation, thus, that $\beta$ coefficient is zero for males. Family history (FHx), cardiovascular disease (CVD).

Table 48. Effects of post intervention perceived stress on post intervention nighttime blood pressure after adjustment for baseline blood pressure, baseline psychosocial functioning and other covariates

\begin{tabular}{|c|c|c|c|c|c|c|c|c|c|c|c|c|}
\hline \multirow[b]{3}{*}{ Predictors } & \multicolumn{4}{|l|}{ All Subjects } & \multicolumn{4}{|l|}{ Females } & \multicolumn{4}{|l|}{ Males } \\
\hline & Systolic & & Diastolic & & Systolic & & Diastolic & & Systolic & & Diastolic & \\
\hline & $\beta$ (SE) & p-value & $\beta$ (SE) & p-value & $\beta$ (SE) & p-value & $\beta$ (SE) & p-value & $\beta$ (SE) & p-value & $\beta(\mathrm{SE})$ & p-value \\
\hline Pre MBSR nighttime BP* & $0.77(0.10)$ & $<.0001$ & $0.82(0.09)$ & $<.0001$ & $0.68(0.12)$ & $<.0001$ & $0.82(0.12)$ & $<.0001$ & $0.77(0.17)$ & 0.001 & $0.79(0.15)$ & $<.0001$ \\
\hline Pre MBSR PS* & $0.06(0.13)$ & 0.68 & $0.02(0.09)$ & 0.83 & $0.41(0.16)$ & 0.01 & $0.12(0.11)$ & 0.28 & $-0.34(0.21)$ & 0.12 & $-0.17(0.16)$ & 0.31 \\
\hline Post MBSR PS & $-0.04(0.18)$ & 0.81 & $0.07(0.11)$ & 0.56 & $-0.52(0.21)$ & 0.02 & $-0.14(0.15)$ & 0.35 & $0.61(0.27)$ & 0.04 & $0.29(0.20)$ & 0.16 \\
\hline Age & $0.00(0.09)$ & 0.99 & $-0.05(0.06)$ & 0.38 & $-0.12(0.11)$ & 0.30 & $-0.11(0.08)$ & 0.19 & $0.26(0.13)$ & 0.07 & $0.09(0.10)$ & 0.38 \\
\hline BMI* & $0.06(0.20)$ & 0.78 & $-0.19(0.13)$ & 0.14 & $-0.10(0.21)$ & 0.65 & $-0.21(0.15)$ & 0.16 & $0.68(0.43)$ & 0.13 & $0.14(0.32)$ & 0.68 \\
\hline Sex (female) & $-2.00(1.98)$ & 0.32 & $-0.47(1.27)$ & 0.71 & & & & & & & & \\
\hline FHx premature CVD (Yes) & $-1.97(2.18)$ & 0.37 & $-2.93(1.41)$ & 0.04 & $-3.24(2.74)$ & 0.25 & $-3.20(1.98)$ & 0.12 & $-0.74(3.26)$ & 0.82 & $-1.01(2.48)$ & 0.69 \\
\hline Drink $\geq 10$ drinks/wk (Yes)* & $5.00(2.49)$ & 0.0497 & $3.51(1.64)$ & 0.04 & $2.73(3.37)$ & 0.42 & $5.09(2.50)$ & 0.0503 & $6.53(3.41)$ & 0.07 & $2.85(2.66)$ & 0.30 \\
\hline Relaxation regularly (Yes)* & $-4.30(2.95)$ & 0.15 & $-3.22(1.91)$ & 0.10 & $-4.71(2.93)$ & 0.12 & $-3.89(2.15)$ & 0.08 & 0.00 & & 0.00 & \\
\hline Race (Causasian) & $0.94(2.71)$ & 0.73 & $-2.74(1.75)$ & 0.12 & $1.46(2.99)$ & 0.63 & $-2.92(2.18)$ & 0.19 & $-2.04(4.14)$ & 0.63 & $-4.85(3.39)$ & 0.17 \\
\hline Education (> high school) & $-0.55(2.65)$ & 0.84 & $-1.34(1.72)$ & 0.44 & $0.43(2.98)$ & 0.89 & $0.04(2.14)$ & 0.99 & $-6.88(5.71)$ & 0.25 & $-7.38(3.91)$ & 0.08 \\
\hline Regular Exercise (Yes)* & $-4.79(3.22)$ & 0.14 & $0.37(2.09)$ & 0.86 & $-1.11(3.79)$ & 0.77 & $-1.43(2.69)$ & 0.60 & $-4.28(6.41)$ & 0.51 & $6.63(4.00)$ & 0.12 \\
\hline
\end{tabular}

* Baseline covariates. NOTE1: only 1 male subject regularly practiced relaxation, thus, that $\beta$ coefficient is zero for males. Family history

(FHx), cardiovascular disease (CVD). 
Table 49. Effects of post intervention anger control inward on post intervention 24-hour blood pressure after adjustment for baseline blood pressure, baseline psychosocial functioning and other covariates

\begin{tabular}{|c|c|c|c|c|c|c|c|c|c|c|c|c|}
\hline \multirow[b]{3}{*}{ Predictors } & \multicolumn{4}{|l|}{ All Subjects } & \multicolumn{4}{|l|}{ Females } & \multicolumn{4}{|l|}{ Males } \\
\hline & Systolic & & Diastolic & & Systolic & & Diastolic & & Systolic & & Diastolic & \\
\hline & $\beta$ (SE) & p-value & $\beta$ (SE) & p-value & $\beta$ (SE) & p-value & $\beta$ (SE) & p-value & $\beta$ (SE) & p-value & $\beta$ (SE) & p-value \\
\hline Pre MBSR 24-hour BP** & $0.87(0.13)$ & $<.0001$ & $1.03(0.12)$ & $<.0001$ & $0.92(0.17)$ & $<.0001$ & $0.92(0.15)$ & $<.0001$ & $0.62(0.30)$ & 0.06 & $1.21(0.27)$ & 0.001 \\
\hline Pre MBSR ACI* & $-0.03(0.20)$ & 0.87 & $-0.02(0.13)$ & 0.89 & $0.11(0.26)$ & 0.67 & $-0.01(0.19)$ & 0.94 & $-0.54(0.43)$ & 0.24 & $-0.33(0.28)$ & 0.27 \\
\hline Post MBSR ACI & $0.23(0.25)$ & 0.36 & $0.15(0.17)$ & 0.37 & $0.37(0.28)$ & 0.21 & $0.36(0.22)$ & 0.11 & $0.36(0.73)$ & 0.63 & $0.14(0.48)$ & 0.77 \\
\hline Age & $-0.08(0.08)$ & 0.36 & $-0.11(0.05)$ & 0.045 & $-0.15(0.10)$ & 0.14 & $-0.12(0.07)$ & 0.10 & $0.12(0.16)$ & 0.45 & $-0.03(0.11)$ & 0.77 \\
\hline BMI* & $0.04(0.18)$ & 0.81 & $-0.13(0.12)$ & 0.26 & $-0.09(0.16)$ & 0.60 & $-0.14(0.12)$ & 0.23 & $0.22(0.66)$ & 0.75 & $0.02(0.43)$ & 0.97 \\
\hline Sex (female) & $-2.88(1.95)$ & 0.15 & $-0.37(1.31)$ & 0.78 & & & & & & & & \\
\hline FHx premature CVD (Yes) & $-2.91(1.97)$ & 0.15 & $-3.21(1.33)$ & 0.02 & $-3.89(2.15)$ & 0.08 & $-3.30(1.64)$ & 0.054 & $-2.82(4.61)$ & 0.55 & $-4.35(3.10)$ & 0.19 \\
\hline Drink $\geq 10$ drinks/wk (Yes)* & $3.43(2.21)$ & 0.13 & $3.18(1.49)$ & 0.04 & $3.84(2.49)$ & 0.13 & $5.02(1.91)$ & 0.01 & $0.62(4.74)$ & 0.90 & $0.18(3.24)$ & 0.96 \\
\hline Relaxation regularly (Yes)* & $-1.26(2.77)$ & 0.65 & $-1.68(1.87)$ & 0.37 & $0.37(2.35)$ & 0.88 & $-1.22(1.78)$ & 0.50 & 0.00 & & 0.00 & \\
\hline Race (Causasian) & $-0.77(2.59)$ & 0.77 & $-2.78(1.75)$ & 0.12 & $1.17(2.42)$ & 0.63 & $-3.07(1.87)$ & 0.11 & $-7.12(5.98)$ & 0.26 & $-2.17(4.71)$ & 0.65 \\
\hline Education (> high school) & $3.76(2.62)$ & 0.16 & $1.38(1.75)$ & 0.43 & $2.23(2.37)$ & 0.35 & $0.82(1.79)$ & 0.65 & $7.42(7.58)$ & 0.35 & $2.98(4.84)$ & 0.55 \\
\hline Regular Exercise (Yes)* & $-5.32(2.88)$ & 0.07 & $-2.64(1.94)$ & 0.18 & $-1.06(2.91)$ & 0.72 & $-1.13(2.16)$ & 0.60 & $-20.53(10.39)$ & 0.07 & $-8.66(5.45)$ & 0.14 \\
\hline
\end{tabular}

* Baseline covariates. NOTE1: only 1 male subject regularly practiced relaxation, thus, that $\beta$ coefficient is zero for males. Family history

(FHx), cardiovascular disease (CVD).

Table 50. Effects of post intervention anger control inward on post intervention daytime blood pressure after adjustment for baseline blood pressure, baseline psychosocial functioning and other covariates

\begin{tabular}{|c|c|c|c|c|c|c|c|c|c|c|c|c|}
\hline \multirow[b]{3}{*}{ Predictors } & \multicolumn{4}{|l|}{ All Subjects } & \multicolumn{4}{|l|}{ Females } & \multicolumn{4}{|l|}{ Males } \\
\hline & Systolic & & Diastolic & & Systolic & & Diastolic & & Systolic & & Diastolic & \\
\hline & $\beta(\mathbf{S E})$ & p-value & $\beta(\mathbf{S E})$ & p-value & $\beta(\mathbf{S E})$ & p-value & $\beta(\mathbf{S E})$ & p-value & $\beta(\mathrm{SE})$ & p-value & $\beta(\mathbf{S E})$ & p-value \\
\hline Pre MBSR daytime BP* & $0.78(0.13)$ & $<.0001$ & $0.91(0.11)$ & $<.0001$ & $0.92(0.16)$ & $<.0001$ & $0.85(0.14)$ & $<.0001$ & $0.39(0.33)$ & 0.27 & $1.03(0.25)$ & 0.001 \\
\hline Pre MBSR ACI* & $-0.07(0.22)$ & 0.75 & $-0.07(0.14)$ & 0.64 & $0.12(0.27)$ & 0.66 & $-0.08(0.21)$ & 0.73 & $-0.62(0.45)$ & 0.19 & $-0.51(0.30)$ & 0.11 \\
\hline Post MBSR ACI & $0.16(0.26)$ & 0.55 & $0.16(0.18)$ & 0.37 & $0.29(0.31)$ & 0.36 & $0.33(0.25)$ & 0.19 & $0.23(0.81)$ & 0.78 & $0.21(0.53)$ & 0.70 \\
\hline Age & $-0.09(0.09)$ & 0.31 & $-0.14(0.06)$ & 0.02 & $-0.18(0.10)$ & 0.08 & $-0.16(0.08)$ & 0.052 & $0.06(0.16)$ & 0.71 & $-0.05(0.11)$ & 0.66 \\
\hline BMI* & $0.06(0.18)$ & 0.74 & $-0.10(0.13)$ & 0.43 & $-0.04(0.17)$ & 0.82 & $-0.11(0.13)$ & 0.43 & $-0.07(0.65)$ & 0.91 & $-0.07(0.46)$ & 0.88 \\
\hline Sex (female) & $-4.56(2.05)$ & 0.03 & $-1.24(1.40)$ & 0.38 & & & & & & & & \\
\hline FHx premature CVD (Yes) & $-2.67(2.08)$ & 0.20 & $-2.94(1.42)$ & 0.04 & $-4.64(2.35)$ & 0.06 & $-3.61(1.83)$ & 0.06 & $-2.95(4.77)$ & 0.55 & $-3.28(3.18)$ & 0.32 \\
\hline Drink $\geq 10$ drinks/wk (Yes)* & $2.96(2.33)$ & 0.21 & $3.04(1.60)$ & 0.06 & $1.89(2.72)$ & 0.49 & $3.72(2.16)$ & 0.10 & $1.22(4.85)$ & 0.81 & $1.13(3.47)$ & 0.75 \\
\hline Relaxation regularly (Yes)* & $1.58(2.92)$ & 0.59 & $0.12(2.02)$ & 0.95 & $3.49(2.51)$ & 0.18 & $0.51(2.00)$ & 0.80 & 0.00 & & 0.00 & \\
\hline Race (Causasian) & $-1.85(2.72)$ & 0.50 & $-2.91(1.89)$ & 0.13 & $0.36(2.62)$ & 0.89 & $-2.81(2.07)$ & 0.19 & $-8.15(5.99)$ & 0.20 & $-3.98(4.91)$ & 0.43 \\
\hline Education (> high school) & $2.51(2.75)$ & 0.37 & $1.48(1.88)$ & 0.44 & $1.93(2.53)$ & 0.45 & $1.16(2.00)$ & 0.57 & $8.21(7.80)$ & 0.31 & $3.03(5.09)$ & 0.56 \\
\hline Regular Exercise (Yes)* & $-5.32(3.05)$ & 0.09 & $-3.39(2.09)$ & 0.11 & $-1.00(3.08)$ & 0.75 & $-1.46(2.45)$ & 0.56 & $-26.64(11.81)$ & 0.04 & $-10.69(5.72)$ & 0.09 \\
\hline
\end{tabular}

* Baseline covariates. NOTE1: only 1 male subject regularly practiced relaxation, thus, that $\beta$ coefficient is zero for males. Family history

(FHx), cardiovascular disease (CVD). 
Table 51. Effects of post intervention anger control inward on post intervention nighttime blood pressure after adjustment for baseline blood pressure, baseline psychosocial functioning and other covariates

\begin{tabular}{|c|c|c|c|c|c|c|c|c|c|c|c|c|}
\hline \multirow[b]{3}{*}{ Predictors } & \multicolumn{4}{|l|}{ All Subjects } & \multicolumn{4}{|l|}{ Females } & \multicolumn{4}{|l|}{ Males } \\
\hline & Systolic & & Diastolic & & Systolic & & Diastolic & & Systolic & & Diastolic & \\
\hline & $\beta(\mathbf{S E})$ & p-value & $\beta(\mathrm{SE})$ & p-value & $\beta(\mathbf{S E})$ & p-value & $\beta(\mathrm{SE})$ & p-value & $\beta(\mathbf{S E})$ & p-value & $\beta(\mathrm{SE})$ & p-value \\
\hline Pre MBSR night BP* & $0.83(0.12)$ & $<.0001$ & $0.84(0.09)$ & $<.0001$ & $0.85(0.21)$ & 0.0003 & $0.79(0.14)$ & $<.0001$ & $0.83(0.20)$ & 0.002 & $0.86(0.20)$ & 0.001 \\
\hline Pre MBSR ACI* ${ }^{*}$ & $0.28(0.22)$ & 0.20 & $0.15(0.14)$ & 0.32 & $0.21(0.36)$ & 0.56 & $-0.03(0.23)$ & 0.90 & $0.25(0.41)$ & 0.55 & $0.15(0.32)$ & 0.64 \\
\hline Post MBSR ACI & $-0.04(0.27)$ & 0.88 & $-0.11(0.18)$ & 0.53 & $0.15(0.39)$ & 0.71 & $0.14(0.26)$ & 0.60 & $-0.32(0.67)$ & 0.65 & $-0.33(0.51)$ & 0.53 \\
\hline Age & $0.00(0.09)$ & 0.98 & $-0.05(0.06)$ & 0.36 & $-0.13(0.13)$ & 0.32 & $-0.08(0.08)$ & 0.31 & $0.28(0.15)$ & 0.08 & $0.09(0.12)$ & 0.48 \\
\hline BMI* & $0.09(0.19)$ & 0.64 & $-0.12(0.12)$ & 0.34 & $-0.19(0.22)$ & 0.41 & $-0.17(0.14)$ & 0.24 & $1.51(0.64)$ & 0.04 & $0.63(0.46)$ & 0.20 \\
\hline Sex (female) & $-2.01(2.11)$ & 0.35 & $-0.15(1.41)$ & 0.92 & & & & & & & & \\
\hline FHx premature CVD (Yes) & $-1.53(2.14)$ & 0.48 & $-2.59(1.42)$ & 0.07 & $-3.98(2.85)$ & 0.17 & $-2.82(1.97)$ & 0.16 & $1.98(4.25)$ & 0.65 & $-0.58(3.27)$ & 0.86 \\
\hline Drink $\geq 10$ drinks/wk (Yes)* & $4.70(2.42)$ & 0.06 & $3.00(1.63)$ & 0.07 & $3.39(2.42)$ & 0.33 & $5.23(2.38)$ & 0.04 & $-1.24(4.46)$ & 0.79 & $-2.08(3.34)$ & 0.54 \\
\hline Relaxation regularly (Yes)* & $-5.49(3.05)$ & 0.08 & $-4.50(2.03)$ & 0.03 & $-2.47(3.47)$ & 0.48 & $-3.91(2.32)$ & 0.10 & 0.00 & & 0.00 & \\
\hline Race (Causasian) & $1.80(2.84)$ & 0.53 & $-2.70(1.87)$ & 0.15 & $3.17(3.27)$ & 0.34 & $-1.88(2.25)$ & 0.41 & $-3.92(5.59)$ & 0.50 & $-5.20(4.41)$ & 0.26 \\
\hline Education (> high school) & $3.33(2.85)$ & 0.25 & $0.54(1.87)$ & 0.77 & $0.22(3.19)$ & 0.94 & $0.34(2.09)$ & 0.87 & $6.89(6.80)$ & 0.33 & $0.61(5.11)$ & 0.91 \\
\hline Regular Exercise (Yes)* & $-5.87(3.14)$ & 0.07 & $-0.50(2.07)$ & 0.81 & $-0.92(4.05)$ & 0.82 & $-1.55(2.55)$ & 0.55 & $-10.88(8.51)$ & 0.23 & $2.18(5.70)$ & 0.71 \\
\hline
\end{tabular}

* Baseline covariates. NOTE1: only 1 male subject regularly practiced relaxation, thus, that $\beta$ coefficient is zero for males. Family history (FHx), cardiovascular disease (CVD). 
Table 52. Effects of post intervention anger control outward on post intervention 24-hour blood pressure after adjustment for baseline blood pressure, baseline psychosocial functioning and other covariates

\begin{tabular}{|c|c|c|c|c|c|c|c|c|c|c|c|}
\hline \multirow[b]{3}{*}{ Predictors } & \multicolumn{4}{|l|}{ All Subjects } & \multicolumn{4}{|l|}{ Females } & \multicolumn{3}{|l|}{ Males } \\
\hline & Systolic & & Diastolic & & Systolic & & Diastolic & & Systolic & Diastolic & \\
\hline & $\beta$ (SE) & p-value & $\beta(\mathrm{SE})$ & p-value & $\beta$ (SE) & p-value & $\beta$ (SE) & p-value & p-value & $\beta$ (SE) & p-value \\
\hline Pre MBSR 24-hour BP** & $0.87(0.13)$ & $<.0001$ & $1.01(0.12)$ & $<.0001$ & $0.97(0.17)$ & $<.0001$ & $0.90(0.15)$ & $<.0001$ & & $1.32(0.27)$ & 0.0003 \\
\hline Pre MBSR ACO** & $-0.04(0.26)$ & 0.89 & $0.01(0.18)$ & 0.97 & $0.39(0.28)$ & 0.17 & $0.31(0.21)$ & 0.16 & & $-0.24(0.44)$ & 0.60 \\
\hline Post MBSR ACO & $0.11(0.27)$ & 0.67 & $0.03(0.18)$ & 0.87 & $-0.38(0.30)$ & 0.22 & $-0.17(0.23)$ & 0.48 & & $-0.15(0.46)$ & 0.74 \\
\hline Age & $-0.08(0.08)$ & 0.36 & $-0.11(0.05)$ & 0.047 & $-0.20(0.10)$ & 0.053 & $-0.16(0.07)$ & 0.03 & & $-0.06(0.11)$ & 0.62 \\
\hline BMI* & $0.03(0.18)$ & 0.87 & $-0.14(0.12)$ & 0.26 & $-0.12(0.16)$ & 0.47 & $-0.15(0.12)$ & 0.21 & & $0.13(0.48)$ & 0.79 \\
\hline Sex (female) & $-2.83(1.97)$ & 0.16 & $-0.31(1.34)$ & 0.82 & & & & & & & \\
\hline FHx premature CVD (Yes) & $-3.21(1.98)$ & 0.11 & $-3.38(1.34)$ & 0.02 & $-2.15(2.27)$ & 0.35 & $-2.27(1.71)$ & 0.20 & & $-4.72(2.99)$ & 0.14 \\
\hline Drink $\geq 10$ drinks/wk (Yes)* & $3.30(2.23)$ & 0.14 & $3.08(1.51)$ & 0.046 & $5.01(2.64)$ & 0.07 & $5.92(2.01)$ & 0.01 & & $-0.05(3.32)$ & 0.99 \\
\hline Relaxation regularly (Yes)* & $-0.86(2.72)$ & 0.75 & $-1.41(1.84)$ & 0.45 & $-1.80(2.34)$ & 0.45 & $-2.79(1.78)$ & 0.13 & & 0.00 & \\
\hline Race (Causasian) & $-1.04(2.60)$ & 0.69 & $-3.00(1.76)$ & 0.09 & $1.11(2.55)$ & 0.67 & $-3.51(1.94)$ & 0.08 & & $-2.57(4.39)$ & 0.57 \\
\hline Education (> high school) & $3.24(2.62)$ & 0.22 & $0.99(1.76)$ & 0.57 & $1.44(2.63)$ & 0.59 & $0.49(2.00)$ & 0.81 & & $5.73(5.75)$ & 0.34 \\
\hline Regular Exercise (Yes)* & $-5.41(3.08)$ & 0.09 & $-2.59(2.08)$ & 0.22 & $-0.29(3.12)$ & 0.93 & $-0.13(2.35)$ & 0.96 & & $-9.49(5.43)$ & 0.11 \\
\hline
\end{tabular}

* Baseline covariates. NOTE1: Grey fill indicate s an overall null model. NOTE2: only 1 male subject regularly practiced relaxation, thus, that $\beta$ coefficient is zero for males. Family history (FHx), cardiovascular disease (CVD).

Table 53. Effects of post intervention anger control outward on post intervention daytime blood pressure after adjustment for baseline blood pressure, baseline psychosocial functioning and other covariates

\begin{tabular}{|c|c|c|c|c|c|c|c|c|c|c|c|}
\hline \multirow[b]{3}{*}{ Predictors } & \multicolumn{4}{|l|}{ All Subjects } & \multicolumn{4}{|l|}{ Females } & \multicolumn{3}{|l|}{ Males } \\
\hline & Systolic & & Diastolic & & Systolic & & Diastolic & & Systolic & Diastolic & \\
\hline & $\beta(\mathbf{S E})$ & p-value & $\beta(\mathbf{S E})$ & p-value & $\beta(\mathbf{S E})$ & p-value & $\beta(\mathbf{S E})$ & p-value & p-value & $\beta(\mathbf{S E})$ & p-value \\
\hline Pre MBSR daytime BP* & $0.78(0.13)$ & $<.0001$ & $0.89(0.11)$ & $<.0001$ & $0.91(0.16)$ & $<.0001$ & $0.78(0.14)$ & $<.0001$ & & $1.13(0.25)$ & 0.001 \\
\hline Pre MBSR ACO* & $-0.04(0.28)$ & 0.89 & $-0.06(0.19)$ & 0.77 & $0.31(0.30)$ & 0.30 & $0.20(0.23)$ & 0.38 & & $-0.59(0.51)$ & 0.27 \\
\hline Post MBSR ACO & $0.08(0.28)$ & 0.79 & $0.11(0.19)$ & 0.56 & $-0.36(0.32)$ & 0.28 & $0.00(0.25)$ & 0.99 & & $0.20(0.50)$ & 0.70 \\
\hline Age & $-0.09(0.09)$ & 0.30 & $-0.14(0.06)$ & 0.02 & $-0.21(0.10)$ & 0.06 & $-0.21(0.08)$ & 0.01 & & $-0.11(0.13)$ & 0.39 \\
\hline BMI* & $0.06(0.19)$ & 0.77 & $-0.11(0.13)$ & 0.39 & $-0.06(0.17)$ & 0.73 & $-0.11(0.13)$ & 0.41 & & $-0.09(0.54)$ & 0.88 \\
\hline Sex (female) & $-4.58(2.06)$ & 0.03 & $-1.30(1.41)$ & 0.36 & & & & & & & \\
\hline FHx premature CVD (Yes) & $-2.81(2.07)$ & 0.18 & $-3.09(1.43)$ & 0.04 & $-2.96(2.44)$ & 0.24 & $-3.20(1.86)$ & 0.10 & & $-3.46(3.23)$ & 0.30 \\
\hline Drink $\geq 10$ drinks/wk (Yes)* & $2.89(2.33)$ & 0.22 & $2.97(1.60)$ & 0.07 & $2.87(2.86)$ & 0.33 & $4.46(2.20)$ & 0.052 & & $2.21(3.72)$ & 0.56 \\
\hline Relaxation regularly (Yes)* & $1.64(2.84)$ & 0.57 & $0.17(1.97)$ & 0.93 & $1.74(2.51)$ & 0.49 & $-0.68(1.93)$ & 0.73 & & 0.00 & \\
\hline Race (Causasian) & $-2.05(2.72)$ & 0.46 & $-3.12(1.89)$ & 0.10 & $0.61(2.71)$ & 0.82 & $-3.46(2.09)$ & 0.11 & & $-4.17(4.75)$ & 0.40 \\
\hline Education (> high school) & $2.22(2.74)$ & 0.42 & $1.24(1.87)$ & 0.51 & $1.05(2.81)$ & 0.71 & $1.41(2.18)$ & 0.52 & & $4.34(6.25)$ & 0.50 \\
\hline Regular Exercise (Yes)* & $-5.41(3.23)$ & 0.10 & $-3.55(2.22)$ & 0.12 & $-0.31(3.32)$ & 0.93 & $-0.86(2.58)$ & 0.74 & & $-10.81(5.96)$ & 0.09 \\
\hline
\end{tabular}

* Baseline covariates. NOTE1: Grey fill indicate s an overall null model. NOTE2: only 1 male subject regularly practiced relaxation, thus, that $\beta$ coefficient is zero for males. Family history (FHx), cardiovascular disease (CVD). 
Table 54. Effects of post intervention anger control outward on post intervention nighttime blood pressure after adjustment for baseline blood pressure, baseline psychosocial functioning and other covariates

\begin{tabular}{|c|c|c|c|c|c|c|c|c|c|c|c|c|}
\hline \multirow[b]{3}{*}{ Predictors } & \multicolumn{4}{|l|}{ All Subjects } & \multicolumn{4}{|l|}{ Females } & \multicolumn{4}{|l|}{ Males } \\
\hline & Systolic & & Diastolic & & Systolic & & Diastolic & & Systolic & & Diastolic & \\
\hline & $\beta(\mathrm{SE})$ & p-value & $\beta$ (SE) & p-value & $\beta(\mathrm{SE})$ & p-value & $\beta(\mathrm{SE})$ & p-value & $\beta(\mathrm{SE})$ & p-value & $\beta$ (SE) & p-value \\
\hline Pre MBSR night BP* & $0.85(0.12)$ & $<.0001$ & $0.85(0.10)$ & $<.0001$ & $0.97(0.17)$ & $<.0001$ & $0.84(0.13)$ & $<.0001$ & $0.91(0.19)$ & 0.001 & $0.97(0.19)$ & 0.0003 \\
\hline Pre MBSR ACO* & $0.14(0.29)$ & 0.63 & $0.09(0.19)$ & 0.66 & $0.41(0.36)$ & 0.26 & $0.25(0.24)$ & 0.32 & $0.96(0.60)$ & 0.13 & $0.54(0.46)$ & 0.26 \\
\hline Post MBSR ACO & $-0.04(0.30)$ & 0.90 & $-0.11(0.20)$ & 0.59 & $-0.35(0.39)$ & 0.37 & $-0.23(0.27)$ & 0.40 & $-0.99(0.61)$ & 0.13 & $-0.80(0.49)$ & 0.13 \\
\hline Age & $0.01(0.09)$ & 0.92 & $-0.04(0.06)$ & 0.45 & $-0.20(0.12)$ & 0.12 & $-0.09(0.08)$ & 0.25 & $0.38(0.14)$ & 0.02 & $0.13(0.11)$ & 0.27 \\
\hline BMI* & $0.08(0.20)$ & 0.70 & $-0.12(0.13)$ & 0.37 & $-0.24(0.21)$ & 0.28 & $-0.17(0.14)$ & 0.22 & $2.05(0.67)$ & 0.01 & $0.93(0.48)$ & 0.08 \\
\hline Sex (female) & $-1.72(2.16)$ & 0.43 & $0.03(1.43)$ & 0.98 & & & & & & & & \\
\hline FHx premature CVD (Yes) & $-1.74(2.18)$ & 0.43 & $-2.59(1.42)$ & 0.07 & $-2.30(2.88)$ & 0.43 & $-2.02(1.97)$ & 0.31 & $3.16(3.64)$ & 0.40 & $-0.27(2.75)$ & 0.92 \\
\hline $\begin{array}{l}\text { Drink } \geq 10 \text { drinks/wk } \\
(\text { Yes)* }\end{array}$ & $4.71(2.47)$ & 0.06 & $3.03(1.65)$ & 0.07 & $4.92(3.49)$ & 0.17 & $6.20(2.45)$ & 0.02 & $-4.35(4.33)$ & 0.33 & $-3.70(3.20)$ & 0.27 \\
\hline Relaxation regularly (Yes)* & $-4.52(3.03)$ & 0.14 & $-4.10(1.98)$ & 0.04 & $-5.22(3.10)$ & 0.10 & $-4.90(2.17)$ & 0.03 & 0.00 & & 0.00 & \\
\hline Race (Causasian) & $1.83(2.88)$ & 0.53 & $-2.55(1.87)$ & 0.18 & $2.51(3.35)$ & 0.46 & $-2.41(2.30)$ & 0.30 & $-4.12(5.03)$ & 0.43 & $-4.93(4.00)$ & 0.24 \\
\hline Education (> high school) & $3.00(3.88)$ & 0.30 & $0.54(1.87)$ & 0.77 & $-0.35(3.36)$ & 0.92 & $-0.51(2.30)$ & 0.82 & $9.37(7.17)$ & 0.22 & $4.84(5.74)$ & 0.42 \\
\hline Regular Exercise (Yes)* & $-5.61(3.40)$ & 0.11 & $-0.32(2.22)$ & 0.89 & $-0.61(4.01)$ & 0.88 & $-0.70(2.64)$ & 0.79 & $-8.05(7.71)$ & 0.32 & $2.41(5.04)$ & 0.64 \\
\hline
\end{tabular}

* Baseline covariates. NOTE1: only 1 male subject regularly practiced relaxation, thus, that $\beta$ coefficient is zero for males. Family history (FHx), cardiovascular disease (CVD). 
Table 55. Effects of post intervention anger expression index on post intervention 24-hour blood pressure after adjustment for baseline blood pressure, baseline psychosocial functioning and other covariates

\begin{tabular}{|c|c|c|c|c|c|c|c|c|c|c|c|}
\hline \multirow[b]{3}{*}{ Predictors } & \multicolumn{4}{|l|}{ All Subjects } & \multicolumn{4}{|l|}{ Females } & \multicolumn{3}{|l|}{ Males } \\
\hline & Systolic & & Diastolic & & Systolic & & Diastolic & & Systolic & Diastolic & \\
\hline & $\beta$ (SE) & p-value & $\beta$ (SE) & p-value & $\beta$ (SE) & p-value & $\beta$ (SE) & p-value & p-value & $\beta$ (SE) & p-value \\
\hline Pre MBSR 24-hour BP** & $0.87(0.14)$ & $<.0001$ & $1.02(0.12)$ & $<.0001$ & $0.95(0.17)$ & $<.0001$ & $0.91(0.16)$ & $<.0001$ & & $1.27(0.26)$ & 0.0004 \\
\hline Pre MBSR AXX* & $0.00(0.08)$ & 0.97 & $-0.01(0.05)$ & 0.85 & $-0.12(0.09)$ & 0.19 & $-0.09(0.07)$ & 0.23 & & $0.13(0.11)$ & 0.27 \\
\hline Post MBSR AXX & $0.00(0.10)$ & 0.97 & $0.00(0.07)$ & 0.98 & $0.16(0.11)$ & 0.17 & $0.06(0.09)$ & 0.48 & & $0.01(0.13)$ & 0.91 \\
\hline Age & $-0.07(0.08)$ & 0.39 & $-0.11(0.05)$ & 0.0504 & $-0.17(0.10)$ & 0.10 & $-0.15(0.07)$ & 0.049 & & $-0.05(0.11)$ & 0.64 \\
\hline BMI* & $0.04(0.18)$ & 0.84 & $-0.14(0.12)$ & 0.27 & $-0.11(0.16)$ & 0.52 & $-0.16(0.12)$ & 0.21 & & $0.07(0.41)$ & 0.86 \\
\hline Sex (female) & $-2.67(1.98)$ & 0.19 & $-0.27(1.34)$ & 0.84 & & & & & & & \\
\hline FHx premature CVD (Yes) & $-3.25(2.00)$ & 0.11 & $-3.37(1.35)$ & 0.02 & $-2.25(2.24)$ & 0.32 & $-2.31(1.71)$ & 0.19 & & $-4.80(2.99)$ & 0.14 \\
\hline Drink $\geq 10$ drinks/wk (Yes)* & $3.27(2.25)$ & 0.15 & $3.11(1.52)$ & 0.046 & $4.78(2.60)$ & 0.08 & $5.67(2.02)$ & 0.01 & & $-0.74(3.02)$ & 0.81 \\
\hline Relaxation regularly (Yes)* & $-0.70(2.85)$ & 0.81 & $-1.46(1.92)$ & 0.45 & $-1.78(2.44)$ & 0.47 & $-2.85(1.90)$ & 0.15 & & 0.00 & \\
\hline Race (Causasian) & $-1.06(2.61)$ & 0.69 & $-3.02(1.76)$ & 0.09 & $1.28(2.48)$ & 0.61 & $-3.12(1.94)$ & 0.12 & & $-1.51(4.35)$ & 0.73 \\
\hline Education (> high school) & $3.17(2.60)$ & 0.23 & 0.99 (1.74) & 0.57 & $1.74(2.54)$ & 0.50 & $0.82(1.95)$ & 0.68 & & $4.57(5.36)$ & 0.41 \\
\hline Regular Exercise (Yes)* & $-5.30(2.94)$ & 0.08 & $-2.56(1.98)$ & 0.20 & $-1.86(2.90)$ & 0.53 & $-1.40(2.21)$ & 0.53 & & $-9.96(5.56)$ & 0.10 \\
\hline
\end{tabular}

* Baseline covariates. NOTE1: Grey fill indicate s an overall null model. NOTE2: only 1 male subject regularly practiced relaxation, thus, that $\beta$ coefficient is zero for males. Family history (FHx), cardiovascular disease (CVD).

Table 56. Effects of post intervention anger expression index on post intervention daytime blood pressure after adjustment for baseline blood pressure, baseline psychosocial functioning and other covariate

\begin{tabular}{|c|c|c|c|c|c|c|c|c|c|c|c|}
\hline \multirow[b]{3}{*}{ Predictors } & \multicolumn{4}{|l|}{ All Subjects } & \multicolumn{4}{|l|}{ Females } & \multicolumn{3}{|l|}{ Males } \\
\hline & Systolic & & Diastolic & & Systolic & & Diastolic & & Systolic & Diastolic & \\
\hline & $\beta(\mathrm{SE})$ & p-value & $\beta(\mathbf{S E})$ & p-value & $\beta(\mathrm{SE})$ & p-value & $\beta(\mathbf{S E})$ & p-value & p-value & $\beta(\mathrm{SE})$ & p-value \\
\hline Pre MBSR daytime BP* & $0.79(0.13)$ & $<.0001$ & $0.90(0.11)$ & $<.0001$ & $0.91(0.16)$ & $<.0001$ & $0.79(0.14)$ & $<.0001$ & & $1.11(0.24)$ & 0.0006 \\
\hline Pre MBSR AXX* & $0.03(0.09)$ & 0.77 & $0.01(0.06)$ & 0.82 & $-0.11(0.09)$ & 0.26 & $-0.08(0.07)$ & 0.31 & & $0.20(0.12)$ & 0.11 \\
\hline Post MBSR AXX & $-0.02(0.10)$ & 0.84 & $-0.03(0.07)$ & 0.68 & $0.16(0.12)$ & 0.18 & $0.02(0.09)$ & 0.80 & & $-0.05(0.14)$ & 0.73 \\
\hline Age & $-0.09(0.09)$ & 0.30 & $-0.14(0.06)$ & 0.02 & $-0.19(0.10)$ & 0.09 & $-0.20(0.08)$ & 0.02 & & $-0.08(0.11)$ & 0.47 \\
\hline BMI* & $0.05(0.19)$ & 0.78 & $-0.11(0.13)$ & 0.41 & $-0.05(0.17)$ & 0.78 & $-0.12(0.13)$ & 0.39 & & $0.00(0.43)$ & 1.00 \\
\hline Sex (female) & $-4.50(2.07)$ & 0.03 & $-1.26(1.42)$ & 0.38 & & & & & & & \\
\hline FHx premature CVD (Yes) & $-2.91(2.09)$ & 0.17 & $-3.09(1.44)$ & 0.04 & $-3.02(2.38)$ & 0.22 & $-2.96(1.85)$ & 0.12 & & $-3.70(3.10)$ & 0.26 \\
\hline Drink $\geq 10$ drinks/wk (Yes)* & $2.80(2.35)$ & 0.24 & $2.94(1.62)$ & 0.08 & $2.78(2.79)$ & 0.33 & $4.39(2.19)$ & 0.06 & & $0.18(3.22)$ & 0.96 \\
\hline Relaxation regularly (Yes)* & $1.92(2.98)$ & 0.52 & $0.22(2.07)$ & 0.92 & $1.66(2.61)$ & 0.53 & $-0.96(2.05)$ & 0.64 & & 0.00 & \\
\hline Race (Causasian) & $-2.00(2.72)$ & 0.47 & $-3.11(1.89)$ & 0.11 & $0.54(2.62)$ & 0.84 & $-3.02(2.07)$ & 0.16 & & $-2.95(4.49)$ & 0.52 \\
\hline Education (> high school) & $2.22(2.71)$ & 0.42 & $1.20(1.86)$ & 0.52 & $1.22(2.67)$ & 0.65 & $1.44(2.12)$ & 0.50 & & $4.29(5.68)$ & 0.47 \\
\hline Regular Exercise (Yes)* & $-5.37(3.08)$ & 0.09 & $-3.37(2.12)$ & 0.12 & $-1.64(3.05)$ & 0.59 & $-1.64(2.41)$ & 0.50 & & $-11.87(5.88)$ & 0.07 \\
\hline
\end{tabular}

* Baseline covariates. NOTE1: Grey fill indicate s an overall null model. NOTE2: only 1 male subject regularly practiced relaxation, thus, that $\beta$ coefficient is zero for males. Family history (FHx), cardiovascular disease (CVD). 
Table 57. Effects of post intervention anger expression index on post intervention nighttime blood pressure after adjustment for baseline blood pressure, baseline psychosocial functioning and other covariates

\begin{tabular}{|c|c|c|c|c|c|c|c|c|c|c|c|c|}
\hline \multirow[b]{3}{*}{ Predictors } & \multicolumn{4}{|l|}{ All Subjects } & \multicolumn{4}{|l|}{ Females } & \multicolumn{4}{|l|}{ Males } \\
\hline & Systolic & & Diastolic & & Systolic & & Diastolic & & Systolic & & Diastolic & \\
\hline & $\beta$ (SE) & p-value & $\beta(\mathrm{SE})$ & p-value & $\beta$ (SE) & p-value & $\beta$ (SE) & p-value & $\beta$ (SE) & p-value & $\beta$ (SE) & p-value \\
\hline Pre MBSR night BP* & $0.85(0.12)$ & $<.0001$ & $0.85(0.10)$ & $<.0001$ & $0.95(0.18)$ & $<.0001$ & $0.83(0.13)$ & $<.0001$ & $0.86(0.20)$ & 0.001 & $0.89(0.19)$ & 0.001 \\
\hline Pre MBSR AXX* & $-0.10(0.09)$ & 0.24 & $-0.05(0.06)$ & 0.40 & $-0.11(0.11)$ & 0.33 & $-0.08(0.08)$ & 0.32 & $-0.12(0.15)$ & 0.44 & $-0.05(0.11)$ & 0.66 \\
\hline Post MBSR AXX & $0.11(0.11)$ & 0.31 & $0.07(0.07)$ & 0.29 & $0.12(0.14)$ & 0.43 & $0.08(0.10)$ & 0.39 & $0.19(0.18)$ & 0.29 & $0.17(0.13)$ & 0.22 \\
\hline Age & $0.02(0.09)$ & 0.87 & $-0.04(0.06)$ & 0.49 & $-0.17(0.13)$ & 0.18 & $-0.08(0.08)$ & 0.31 & $0.30(0.14)$ & 0.053 & $0.10(0.11)$ & 0.41 \\
\hline BMI* & $0.10(0.20)$ & 0.61 & $-0.11(0.13)$ & 0.40 & $-0.22(0.21)$ & 0.31 & $-0.16(0.14)$ & 0.24 & $1.51(0.58)$ & 0.02 & $0.59(0.41)$ & 0.18 \\
\hline Sex (female) & $-1.42(2.14)$ & 0.51 & $0.15(1.42)$ & 0.92 & & & & & & & & \\
\hline FHx premature CVD (Yes) & $-1.62(2.16)$ & 0.46 & $-2.55(1.41)$ & 0.08 & $-2.50(2.86)$ & 0.39 & $-2.10(1.92)$ & 0.28 & $2.40(3.85)$ & 0.54 & $-0.38(2.87)$ & 0.90 \\
\hline Drink $\geq 10$ drinks/wk (Yes)* & $4.94(2.46)$ & 0.0499 & $3.14(1.65)$ & 0.06 & $4.49(3.44)$ & 0.20 & $6.02(2.39)$ & 0.02 & $-1.16(4.05)$ & 0.78 & $-1.99(3.04)$ & 0.53 \\
\hline Relaxation regularly (Yes)* & $-4.94(3.13)$ & 0.12 & $-4.26(2.07)$ & 0.045 & $-5.19(3.28)$ & 0.13 & $-5.02(2.29)$ & 0.04 & 0.00 & & 0.00 & \\
\hline Race (Causasian) & $1.60(2.84)$ & 0.58 & $-2.65(1.86)$ & 0.16 & $2.83(3.30)$ & 0.40 & $-2.32(2.26)$ & 0.31 & $-3.61(5.08)$ & 0.49 & $-4.35(4.06)$ & 0.31 \\
\hline Education (> high school) & $2.93(2.82)$ & 0.30 & $0.47(1.83)$ & 0.80 & $0.19(3.29)$ & 0.95 & $-0.18(2.17)$ & 0.93 & $8.58(7.21)$ & 0.26 & $2.93(5.44)$ & 0.60 \\
\hline Regular Exercise (Yes)* & $-5.68(3.20)$ & 0.08 & $-0.45(2.09)$ & 0.83 & $-2.16(3.78)$ & 0.57 & $-1.68(2.46)$ & 0.50 & $-10.36(8.29)$ & 0.24 & $1.76(5.46)$ & 0.75 \\
\hline
\end{tabular}

* Baseline covariates. NOTE1: only 1 male subject regularly practiced relaxation, thus, that $\beta$ coefficient is zero for males. Family history (FHx), cardiovascular disease (CVD). 
Table 58. Standardized and unstandardized effects of post intervention primary predictor variables on post intervention 24-hour blood pressure for all subjects after adjustment for baseline blood pressure, baseline psychosocial functioning and other covariates

\begin{tabular}{|lll|lr|}
\hline & \multicolumn{1}{l}{ Systolic Blood Pressure } & & Diastolic Blood Pressure \\
\cline { 2 - 4 } Predictors & $\boldsymbol{\beta}(\mathrm{SE})$ Unstandardized & $\boldsymbol{\beta}(\mathrm{SE})$ Standardized & $\boldsymbol{\beta}(\mathrm{SE})$ Unstandardized & $\boldsymbol{\beta}$ (SE) Standardized \\
T-Ang & $0.11(0.39)$ & $0.05(0.18)$ & $-0.01(0.26)$ & $-0.01(0.14)$ \\
T-Ang/T & $1.14(0.82)$ & $0.25(0.18)$ & $0.40(0.56)$ & $0.10(0.14)$ \\
T-Ang/R & $0.09(0.61)$ & $0.02(0.16)$ & $0.04(0.41)$ & $0.01(0.13)$ \\
AXI & $0.30(0.24)$ & $0.13(0.11)$ & $0.21(0.17)$ & $0.11(0.09)$ \\
AXO & $0.23(0.40)$ & $0.07(0.12)$ & $0.00(0.27)$ & $0.00(0.09)$ \\
PS & $-0.03(0.16)$ & $-0.02(0.11)$ & $-0.06(0.11)$ & $-0.05(0.09)$ \\
\hline
\end{tabular}

T-Ang= Trait Anger, T-Ang/T = Trait Anger Temperament, $\mathrm{T}-\mathrm{Ang} / \mathrm{R}=$ Trait Anger Reaction, $\mathrm{AXI}=$ Anger Expression Inwards, $\mathrm{AXO}=$ Anger Expression Outwards, PS = Perceived Stress. 
Table 59. Standardized and unstandardized effects of post intervention primary predictor variables on post intervention daytime blood pressure for all subjects after adjustment for baseline blood pressure, baseline psychosocial functioning and other covariates

\begin{tabular}{|lll|ll|}
\hline & \multicolumn{1}{l}{ Systolic Blood Pressure } & & Diastolic Blood Pressure \\
\cline { 2 - 4 } Predictors & $\boldsymbol{\beta}(\mathrm{SE})$ Unstandardized & $\boldsymbol{\beta}(\mathrm{SE})$ Standardized & $\boldsymbol{\beta}($ SE) Unstandardized & $\boldsymbol{\beta}$ (SE) Standardized \\
T-Ang & $-0.03(0.41)$ & $-0.02(0.18)$ & $-0.10(0.28)$ & $-0.05(0.14)$ \\
T-Ang/T & $0.77(0.86)$ & $0.16(0.18)$ & $0.31(0.60)$ & $0.07(0.14)$ \\
T-Ang/R & $0.08(0.63)$ & $0.02(0.16)$ & $-0.04(0.44)$ & $-0.01(0.13)$ \\
AXI & $0.12(0.26)$ & $0.05(0.11)$ & $0.11(0.18)$ & $0.05(0.09)$ \\
AXO & $0.06(0.42)$ & $0.02(0.12)$ & $-0.05(0.28)$ & $-0.02(0.09)$ \\
PS & $-0.05(0.17)$ & $-0.04(0.12)$ & $-0.10(0.12)$ & $-0.08(0.09)$ \\
\hline
\end{tabular}

T-Ang= Trait Anger, T-Ang/T = Trait Anger Temperament, $\mathrm{T}-\mathrm{Ang} / \mathrm{R}=$ Trait Anger Reaction, $\mathrm{AXI}=$ Anger Expression Inwards, $\mathrm{AXO}=$ Anger Expression Outwards, PS = Perceived Stress. 
Table 60. Baseline demographic data by sex

\begin{tabular}{|lcc|}
\hline Characteristics & $\begin{array}{c}\text { Male } \\
(\mathbf{n}=\mathbf{3 8})\end{array}$ & Female (n=63) \\
\hline Age $($ years) & $56(12.4)$ & $56(10.9)$ \\
Waist circ. $(\mathrm{cm})$ & $96(8.9)$ & $87(11.8)$ \\
BMI $\left(\mathrm{kg} / \mathrm{m}^{\wedge} 2\right)$ & $28(3.2)$ & $28(5.6)$ \\
Office SBP & $133(13.2)$ & $137(12.7)$ \\
Office DBP & $85(7.3)$ & $86(7.1)$ \\
24 HR SBP & $134(7.3)$ & $135(8.3)$ \\
24 HR DBP & $82(4.7)$ & $82(6.3)$ \\
Daytime SBP & $140(7.6)$ & $141(7.9)$ \\
Daytime DBP & $87(5.4)$ & $87(6.8)$ \\
Nighttime SBP & $119(9.6)$ & $123(11.6)$ \\
Nighttime DBP & $70(7.2)$ & $72(7.6)$ \\
Family History of Hypertension (\%) & $64 \%$ & $62 \%$ \\
Family history of premature coronary heart disease & $25 \%$ & $30 \%$ \\
$\geq$ Post-Secondary Education (\% yes) & $86 \%$ & $85 \%$ \\
Working (\%) & $83 \%$ & $77 \%$ \\
Drinks $>$ 10/week & $33 \%$ & $11 \%$ \\
Use relaxation techniques on a regular basis & $3 \%$ & $25 \%$ \\
Vigorous exercise & $78 \%$ & $61 \%$ \\
Moderate exercise & $92 \%$ & $91 \%$ \\
Self-identify as Caucasian & $86 \%$ & $79 \%$ \\
\hline Data is reported in mean (SD) for continuous variables. SBP systolic blood pressure, DBP $=$ \\
diastolic blood pressure. All BP measured in mmHg. Vigorous exercise= participation in any \\
weekly vigorous aerobic exercise lasting 20 $\geq$ minutes. Moderate exercise = participation in \\
any weekly moderate exercise lasting $\geq$ 30 minutes. & \\
\hline
\end{tabular}


Table 61. Baseline psychosocial data by sex

\begin{tabular}{|c|c|c|c|c|}
\hline Psychometric Scale & $\begin{array}{l}\text { Score } \\
\text { Males }\end{array}$ & $\begin{array}{c}\text { \%ile for } \\
\text { Normal Male } \\
\text { Adults** }\end{array}$ & $\begin{array}{l}\text { Score } \\
\text { Females }\end{array}$ & $\begin{array}{c}\text { \%ile for Normal } \\
\text { Female } \\
\text { Adults*** }\end{array}$ \\
\hline State Anger & $17(4.3)$ & $60^{\text {th }}$ & $16(3.9)$ & $55^{\text {th }}$ \\
\hline Feeling angry & $6(1.9)$ & $50^{\text {th }}$ & $6(2.0)$ & $55^{\text {th }}$ \\
\hline $\begin{array}{l}\text { Feel like expressing } \\
\text { anger verbally }\end{array}$ & $6(2.3)$ & $75^{\text {th }}$ & $5(0.9)$ & $50^{\text {th }}$ \\
\hline $\begin{array}{l}\text { Feel like expressing } \\
\text { anger physically }\end{array}$ & $5(0.4)$ & $50^{\text {th }}$ & $5(1.3)$ & $50^{\text {th }}$ \\
\hline Trait Anger & $18(6.3)$ & $60^{\text {thl }}$ & $17(4.5)$ & $55^{\text {th }}$ \\
\hline Angry Temperament & $7(3.1)$ & $70^{\text {th }}$ & $6(2.3)$ & $60^{\text {th }}$ \\
\hline Angry Reaction & $8(3.0)$ & $45^{\text {th }}$ & $8(2.3)$ & $40^{\text {th }}$ \\
\hline Anger Expression In & $18(4.5)$ & $90^{\text {th }}$ & $16(4.3)$ & $80^{\text {th }}$ \\
\hline Anger Expression Out & $14(3.7)$ & $40^{\text {th }}$ & $13(3.0)$ & $30^{\text {th }}$ \\
\hline Anger Control In & $20(5.4)$ & $20^{\text {th }}$ & $23(5.6)$ & $35^{\text {th }}$ \\
\hline Anger Control Out & $22(4.8)$ & $45^{\text {th }}$ & $24(5.4)$ & $50^{\text {th }}$ \\
\hline $\begin{array}{l}\text { Anger Expression Index (AX } \\
\text { index) }\end{array}$ & $38(13.0)$ & $70^{\text {th }}$ & $31(14.6)$ & $55^{\text {th }}$ \\
\hline Perceived Stress Scale & $25(7.8)$ & N/A & $23(8.4)$ & N/A \\
\hline
\end{tabular}


Table 62. Primary results summary: associations between primary post intervention anger measures and post intervention 24-hour blood pressure after adjustment for baseline blood pressure, baseline psychosocial functioning and other covariates

\begin{tabular}{|llllll|}
\hline & All Subjects & & Females & Males \\
\cline { 2 - 5 } & Systolic & Diastolic & Systolic & Diastolic & Systolic \\
\hline Trait Anger & No sig assoc. & No sig assoc. & No sig assoc. & No sig assoc. & Diastolic \\
Trait Anger Temperament & No sig assoc. & No sig assoc. & Sig positive assoc. & No sig assoc. \\
Trait Anger Reaction & No sig assoc. & No sig assoc. & No sig assoc. & No sig assoc. \\
Anger Expression Inwards & No sig assoc. & No sig assoc. & No sig assoc. & No sig assoc. & No sig assoc. \\
Anger Expression Outwards & No sig assoc. & No sig assoc. & No sig assoc. & No sig assoc. & No sig assoc. \\
Perceived Stress & No sig assoc. & No sig assoc. & Sig negative assoc. & No sig assoc. & Sig positive assoc. \\
\hline
\end{tabular}

NOTE: Grey fill indicate s an overall null model.

Table 63. Primary results summary: associations between primary post intervention anger measures and post intervention daytime blood pressure after adjustment for baseline blood pressure, baseline psychosocial functioning and other covariates

\begin{tabular}{|llllll|}
\hline & All Subjects & \multicolumn{2}{c|}{ Males } \\
\cline { 2 - 6 } & Systolic & Diastolic & Systolic & Diastolic & Systolic \\
\hline Trait Anger & No sig assoc. & No sig assoc. & No sig assoc. & No sig assoc. & \\
Trait Anger Temperament & No sig assoc. & No sig assoc. & No sig assoc. & No sig assoc. \\
Trait Anger Reaction & No sig assoc. & No sig assoc. & No sig assoc. & No sig assoc. & \\
Anger Expression Inwards & No sig assoc. & No sig assoc. & No sig assoc. & No sig assoc. & No sig assoc. \\
Anger Expression Outwards & No sig assoc. & No sig assoc. & No sig assoc. & No sig assoc. & Sig positive assoc. \\
Perceived Stress & No sig assoc. & No sig assoc. & No sig assoc. & No sig assoc. & Sig assoc. \\
\hline
\end{tabular}

NOTE: Grey fill indicate s an overall null model. 
Table 64. Secondary results summary: associations between post intervention primary anger measures and post intervention nighttime blood pressure after adjustment for baseline blood pressure, baseline psychosocial functioning and other covariates

\begin{tabular}{|c|c|c|c|c|c|c|}
\hline & \multicolumn{2}{|l|}{ All Subjects } & \multicolumn{2}{|l|}{ Females } & \multicolumn{2}{|l|}{ Males } \\
\hline & Systolic & Diastolic & Systolic & Diastolic & Systolic & Diastolic \\
\hline Trait Anger & No sig assoc. & No sig assoc. & No sig assoc. & No sig assoc. & No sig assoc. & No sig assoc. \\
\hline Trait Anger Temperament & No sig assoc. & No sig assoc. & No sig assoc. & No sig assoc. & No sig assoc. & No sig assoc. \\
\hline Trait Anger Reaction & No sig assoc. & No sig assoc. & No sig assoc. & No sig assoc. & No sig assoc. & No sig assoc. \\
\hline Anger Expression Inwards & No sig assoc & No sig assoc. & No sig assoc. & No sig assoc. & No sig assoc. & No sig assoc. \\
\hline Anger Expression Outwards & No sig assoc. & No sig assoc. & No sig assoc. & No sig assoc. & No sig assoc. & No sig assoc. \\
\hline Perceived Stress & No sig assoc. & No sig assoc. & Sig negative assoc. & No sig assoc. & Sig positive assoc. & No sig assoc. \\
\hline
\end{tabular}

Table 65. Exploratory results summary: associations between post intervention exploratory anger measures and post intervention 24-hour blood pressure after adjustment for baseline blood pressure, baseline psychosocial functioning and other covariates

\begin{tabular}{|c|c|c|c|c|c|c|}
\hline & \multicolumn{2}{|l|}{ All Subjects } & \multicolumn{2}{|l|}{ Females } & \multicolumn{2}{|l|}{ Males } \\
\hline & Systolic & Diastolic & Systolic & Diastolic & Systolic & Diastolic \\
\hline $\begin{array}{l}\text { Anger Control Inwards } \\
\text { Anger Control Outwards } \\
\text { Anger Expression Index }\end{array}$ & $\begin{array}{l}\text { No sig assoc. } \\
\text { No sig assoc. } \\
\text { No sig assoc. }\end{array}$ & $\begin{array}{l}\text { No sig assoc. } \\
\text { No sig assoc. } \\
\text { No sig assoc. }\end{array}$ & $\begin{array}{l}\text { No sig assoc. } \\
\text { No sig assoc. } \\
\text { No sig assoc. }\end{array}$ & $\begin{array}{l}\text { No sig assoc. } \\
\text { No sig assoc. } \\
\text { No sig assoc. }\end{array}$ & No sig assoc. & $\begin{array}{l}\text { No sig assoc. } \\
\text { No sig assoc. } \\
\text { No sig assoc. }\end{array}$ \\
\hline
\end{tabular}

NOTE: Grey fill indicate s an overall null model. 
Table 66. Exploratory results summary: associations between post intervention exploratory anger measures and post intervention daytime blood pressure after adjustment for baseline blood pressure, baseline psychosocial functioning and other covariates

\begin{tabular}{|llllll|}
\hline & All Subjects & & Females & \multicolumn{2}{c|}{ Males } \\
\cline { 2 - 5 } & Systolic & Diastolic & Systolic & Diastolic & Systolic \\
\hline Anger Control Inwards & No sig assoc. & No sig assoc. & No sig assoc. & No sig assoc. & No sig assoc. \\
Anger Control Outwards & No sig assoc. & No sig assoc. & No sig assoc. & No sig assoc. & No sig assoc. \\
Anger Expression Index & No sig assoc. & No sig assoc. & No sig assoc. & No sig assoc. & No sig assoc. \\
\hline
\end{tabular}

NOTE: Grey fill indicate s an overall null model.

Table 67. Exploratory results summary: associations between post intervention exploratory anger measures and post intervention nighttime blood pressure after adjustment for baseline blood pressure, baseline psychosocial functioning and other covariates

\begin{tabular}{|c|c|c|c|c|c|c|}
\hline & \multicolumn{2}{|l|}{ All Subjects } & \multicolumn{2}{|l|}{ Females } & \multicolumn{2}{|l|}{ Males } \\
\hline & Systolic & Diastolic & Systolic & Diastolic & Systolic & Diastolic \\
\hline
\end{tabular}


Table 68. Standardized and unstandardized effects of post intervention trait anger temperament on post intervention 24-hour systolic blood pressure for female subjects after adjustment for baseline blood pressure, baseline psychosocial functioning and other covariates

\begin{tabular}{|lll|}
\hline & 24-hour Systolic & \\
\cline { 2 - 3 } Predictor & $\begin{array}{l}\boldsymbol{\beta}(\mathrm{SE}) \\
\text { Unstandardized }\end{array}$ & $\begin{array}{l}\boldsymbol{\beta}(\mathrm{SE}) \\
\text { Standardized }\end{array}$ \\
\hline $\begin{array}{l}\text { Trait anger } \\
\text { temperament }\end{array}$ & $2.71(0.95)$ & $0.62(0.22)$ \\
\hline
\end{tabular}

Table 69. Standardized and unstandardized effects of post intervention perceived stress on post intervention systolic blood pressure for female subjects after adjustment for baseline blood pressure, baseline psychosocial functioning and other covariates

\begin{tabular}{|lll|ll|}
\hline & $\mathbf{2 4}$-hour Systolic & Nighttime Systolic \\
\cline { 2 - 5 } Predictor & $\begin{array}{l}\boldsymbol{\beta}(\mathrm{SE}) \\
\text { Unstandardized }\end{array}$ & $\begin{array}{l}\boldsymbol{\beta}(\mathrm{SE}) \\
\text { Standardized }\end{array}$ & $\begin{array}{l}\boldsymbol{\beta}(\mathrm{SE}) \\
\text { Unstandardized }\end{array}$ & $\begin{array}{l}\boldsymbol{\beta}(\mathrm{SE}) \\
\text { Standardized }\end{array}$ \\
\hline $\begin{array}{l}\text { Perceived } \\
\text { Stress }\end{array}$ & $-0.35(0.15)$ & $-0.30(0.13)$ & $-0.52(0.21)$ & $-0.36(0.14)$ \\
\hline
\end{tabular}

Table 70. Standardized and unstandardized effects of post intervention perceived stress on post intervention systolic blood pressure for male subjects after adjustment for baseline blood pressure, baseline psychosocial functioning and other covariates

\begin{tabular}{|lll|ll|l|l|}
\hline & 24-hour Systolic & Daytime Systolic & Nighttime Systolic \\
\cline { 2 - 6 } Predictor & $\begin{array}{l}\boldsymbol{\beta}(\mathrm{SE}) \\
\text { Unstandardized }\end{array}$ & $\begin{array}{l}\boldsymbol{\beta}(\mathrm{SE}) \\
\text { Standardized }\end{array}$ & $\begin{array}{l}\boldsymbol{\beta} \text { (SE) } \\
\text { Unstandardized }\end{array}$ & $\begin{array}{l}\boldsymbol{\beta}(\mathrm{SE}) \\
\text { Standardized }\end{array}$ & $\begin{array}{l}\boldsymbol{\beta}(\mathrm{SE}) \\
\text { Unstandardized }\end{array}$ & $\begin{array}{l}\boldsymbol{\beta}(\mathrm{SE}) \\
\text { Standardized }\end{array}$ \\
\hline $\begin{array}{l}\text { Perceived } \\
\text { Stress }\end{array}$ & $0.83(0.32)$ & $0.49(0.19)$ & $0.91(0.36)$ & $0.52(0.21)$ & $0.61(0.27)$ & $0.33(0.15)$ \\
\hline
\end{tabular}




\section{Figures}

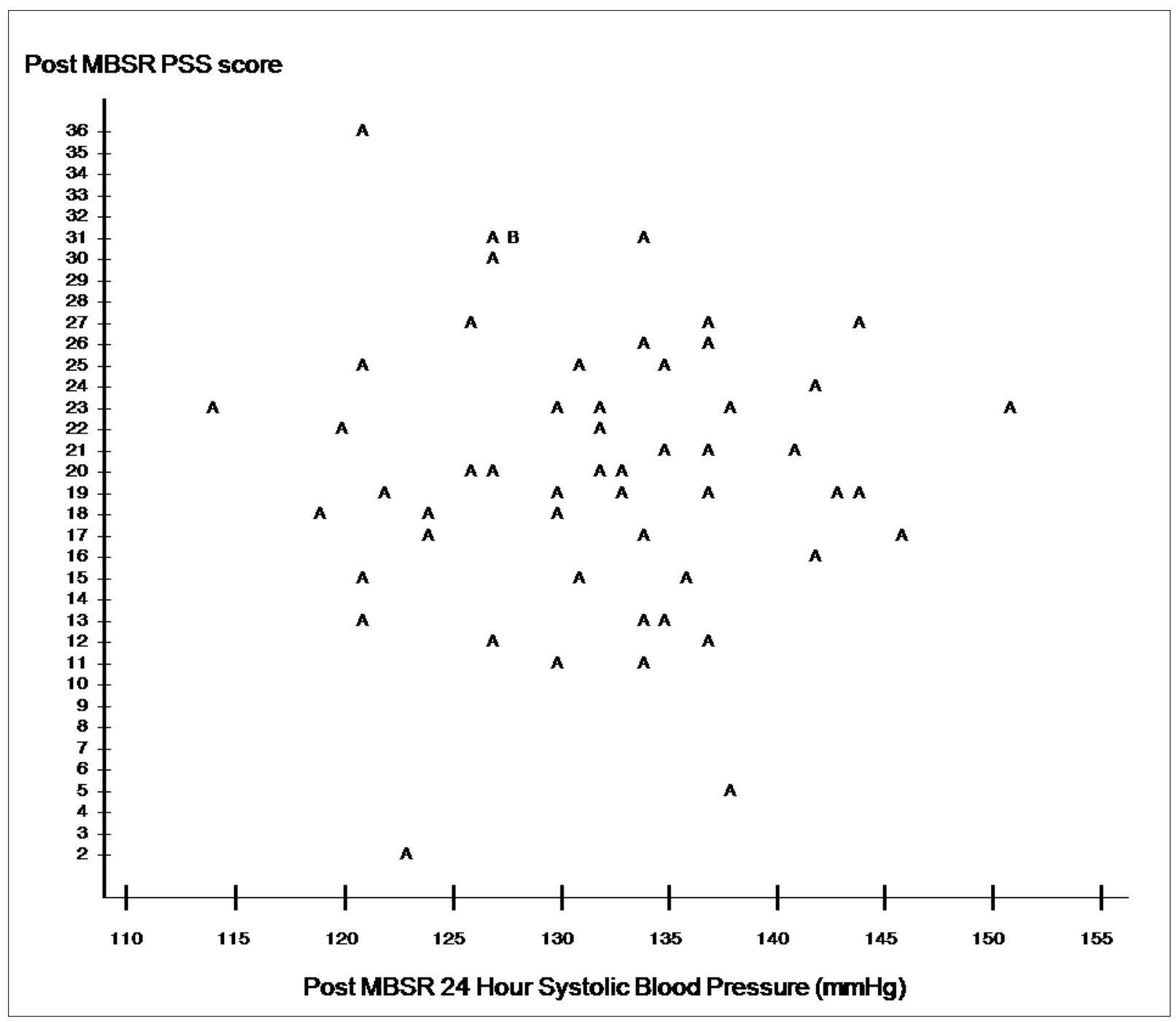

Figure 1. Scatter plot of post intervention 24-hour systolic blood pressure correlated with post intervention perceived stress scale score for female participants. Legend: A = 1 observation, $B$ $=2$ observations 


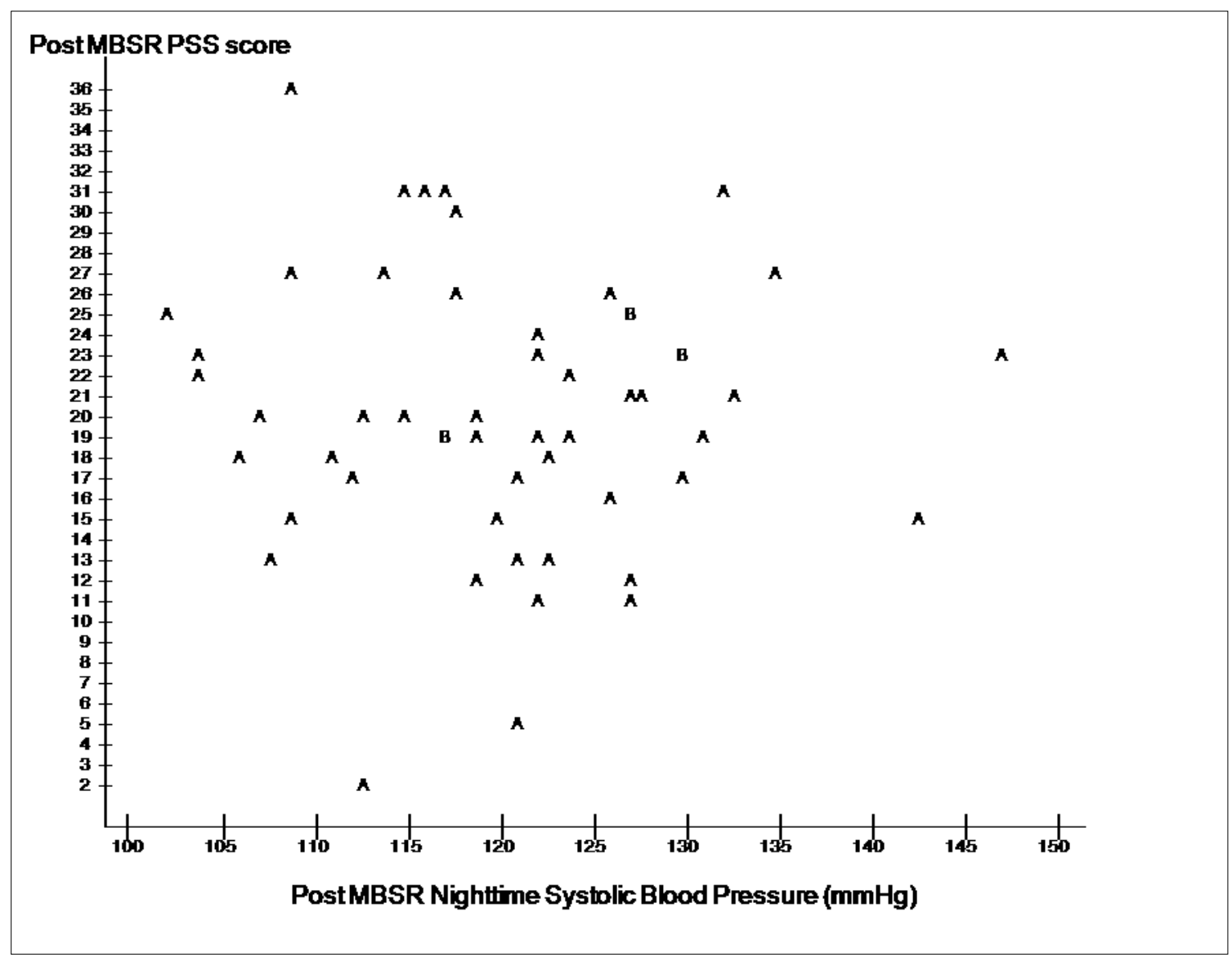

Figure 2. Scatter plot of post intervention nighttime systolic blood pressure correlated with post intervention perceived stress scale score for female participants. Legend: $A=1$ observation, $\mathrm{B}=2$ observations 


\section{References}

1. Blom K, How M, Dai M, Baker B, Irvine J, Abbey S, Abramson BL, Myers M, Perkins N, Tobe SW. Hypertension Analysis of stress Reduction using Mindfulness meditatiON and Yoga (The HARMONY Study): study protocol of a randomised control trial. BMJ Open. 2012;2.

2. Spielberger CD. Manual for the State-Trait Anger Expression Inventory-2. 3rd ed. Odessa, Florida: Psychological Assessment Resources; 1999.

3. Doyle J, Kinch A, Dawber T, Kahn H, Kannel W. Cigarette Smoking and Coronary Heart Disease - Combined Albany-Framingham Study. Circulation. 1961;24:921-\&.

4. Lewington S, Clarke R, Qizilbash N, Peto R, Collins R, Prospective Studies Collaboration. Age-specific relevance of usual blood pressure to vascular mortality: a meta-analysis of individual data for one million adults in 61 prospective studies. Lancet. 2002;360:1903-1913.

5. Ezzati M, Lopez AD, Rodgers A, Vander Hoorn S, Murray CJ, Comparative Risk Assessment Collaborating G. Selected major risk factors and global and regional burden of disease. Lancet. 2002;360:1347-1360.

6. World Health Organization. Preventing chronic diseases: a vital investment. Available at: Www.who.int/chp/chronic_disease_report/contents/en/index.html. Accessed 04/27, 2007.

7. Wolf-Maier K, Cooper R, Kramer H, Banegas J, Giampaoli S, Joffres M, Poulter N, Primatesta P, Stegmayr B, Thamm M. Hypertension treatment and control in five European Countries, Canada, and the United States. Hypertension. 2004;43:10-17.

8. Wolf-Maier K, Cooper R, Banegas J, Giampaoli S, Hense H, Joffres M, Kastarinen M, Poulter N, Primatesta P, Rodriguez-Artalejo F, Stegmayr B, Thamm M, Tuomilehto J, Vanuzzo D, Vescio F. Hypertension, prevalence and blood pressure levels in 6 European countries, Canada, and the United States. JAMA. 2003;289:2363-2369.

9. Wilkins K, Campbell NRC, Joffres MR, McAlister FA, Nichol M, Quach S, Johansen HL, Tremblay MS. Blood pressure in Canadian adults. Health Reports. 2010;21. 
10. Kearney PM, Whelton M, Reynolds K, Muntner P, Whelton PK, He J. Global burden of hypertension: analysis of worldwide data. Lancet. 2005;365:217-223.

11. Williams RR, Hunt SC, Hasstedt SJ, Hopkins PN, Wu LL, Berry TD, Stults BM, Barlow GK, Schumacher MC, Lifton RP, Lalouel JM. Are there Interactions and Relations between Genetic and Environmental-Factors Predisposing to High Blood-Pressure. Hypertension. 1991;18:29-37.

12. Chobanian A, Bakris G, Black H, Cushman W, Green L, Izzo J, Jones D, Materson B, Oparil S, Wright J, Roccella E, Natl High Blood Pressure Educ Prog. Seventh Report of the Joint National Committee on Prevention, Detection, Evaluation, and Treatment of High Blood Pressure. Hypertension. 2003;42:1206-1252.

13. Spruill TM. Chronic Psychosocial Stress and Hypertension. Curr Hypertens Rep. 2010;12:10-16.

14. Sparrenberger F, Cichelero FT, Ascoli AM, Fonseca FP, Weiss G, Berwanger O, Fuchs SC, Moreira LB, Fuchs FD. Does psychosocial stress cause hypertension? A systematic review of observational studies. J Hum Hypertens. 2009;23:12-19.

15. Steptoe A. Psychosocial factors in the development of hypertension. Ann Med. 2000;32:371375.

16. Kaplan M, Nunes A. The psychosocial determinants of hypertension. Nutr Metab Carbiovasc Dis. 2003;13:52-59.

17. Khan NA, Hernmelgarn B, Herman RJ, Bell CM, Mahon JL, Leiter LA, Rahkin SW, Hill MD, Padwal R, Touyz RM, Larochelle P, Feldman RD, Schiffrin EL, Campbell NRC, Moe G, Prasad R, Arnold MO, Campbell TS, Milot A, Stone JA, Jones C, Ogilvie RI, Hamet P, Fodor G, Carruthers G, Burns KD, Ruzicka M, deChamplain J, Pylypchuk G, Petrella R, Boulanger J, Trudeau L, Hegele RA, Woo V, McFarlane P, Vallee M, Howlett J, Bacon SL, Lindsay P, Gilbert RE, Lewanczuk RZ, Tobe S, Canadian Hypertension Educ Program. The 2009 Canadian Hypertension Education Program recommendations for the management of hypertension: Part 2therapy. Can J Cardiol. 2009;25:287-298. 
18. Khan NA, Hemmelgarn B, Padwal R, Larochelle P, Mahon JL, Lewanczuk RZ, McAlister FA, Rabkin SW, Hill MD, Feldman RD, Schiffrin EL, Campbell NRC, Logan AG, Arnold M, Moe G, Campbell TS, Milot A, Stone JA, Jones C, Leiter LA, Ogilvie RI, Herman R, Hamet P, Fodor G, Carruthers G, Culleton B, Burns KD, Ruzicka M, deChamplain J, Pylypchuk G, Gledhill N, Petrella R, Boulanger J, Trudeau L, Hegele RA, Woo V, McFarlane P, Touyz RM, Tobe SW, Canadian Hypertension Educ Program. The 2007 Canadian Hypertension Education Program recommendations for the management of hypertension: Part 2 - therapy. Can J Cardiol. 2007;23:539-550.

19. Yusuf S, Hawken S, Ounpuu S, Dans T, Avezum A, Lanas F, McQueen M, Budaj A, Pais P, Varigos J, Liu L, INTERHEART Study Investigators. Effect of potentially modifiable risk factors associated with myocardial infarction in 52 countries (the INTERHEART study): casecontrol study. Lancet. 2004;364:937-952.

20. Rozanski A, Blumenthal J, Davidson K, Saab P, Kubzansky L. The epidemiology, pathophysiology and management of psychosocial risk factors in cardiac practice - The emerging field of behavioral cardiology. J Am Coll Cardiol. 2005;45:637-651.

21. Heart \& Stroke Foundation. Reduce your stress. Available at: http://www.heartandstroke.com/site/c.ikIQLcMWJtE/b.3484039/k.3548/Heart_disease_Reduce _your_stress.htm. Accessed 09, 2012.

22. Canadian Psychological Association. Heart Disease."Psychology Works" Fact Sheet: Psychological Risk Factors and Heart Disease. Available at: http://www.cpa.ca/psychologyfactsheets/heartdisease/. Accessed 09, 2012.

23. Rozanski A, Blumenthal J, Kaplan J. Impact of psychological factors on the pathogenesis of cardiovascular disease and implications for therapy. Circulation. 1999;99:2192-2217.

24. Igna CV, Julkunen J, Vanhanen H. Anger expression styles and blood pressure: Evidence for different pathways. J Hypertens. 2009;27:1972-1979.

25. Dimsdale JE, Moss J. Short-Term Catecholamine Response to Psychological Stress. Psychosom Med. 1980;42:493-497. 
26. Cacioppo JT, Berntson GG, Malarkey WB, Kiecolt-Glaser JK, Sheridan JF, Poehlmann KM, Burleson MH, Ernst JM, Hawkley LC, Glaser R. Autonomic, neuroendocrine, and immune responses to psychological stress: the reactivity hypothesis. Ann N Y Acad Sci. 1998;840:664673.

27. Powell LH, Lovallo WR, Matthews KA, Meyer P, Midgley AR, Baum A, Stone AA, Underwood L, McCann JJDNS, Janikula Herro KMFA, Ory MG. Physiologic Markers of Chronic Stress in Premenopausal, Middle-Aged Women. Psychosom Med. 2002;64:502-509.

28. Kosten TR, Mason JW, Giller EL, Ostroff RB, Harkness L. Sustained urinary norepinephrine and epinephrine elevation in post-traumatic stress disorder. Psychoneuroendocrinology. 1987;12:13-20.

29. Esler M, Julius S, Zweifler A, Randall O, Harburg E, Gardiner H, DeQuattro V. Mild HighRenin Essential Hypertension. N Engl J Med. 1977;296:405-411.

30. Katholi RE, Carey RM, Ayers CR, Vaughan ED, Yancey MR, Morton CL. Production of Sustained Hypertension by Chronic Intrarenal Norepinephrine Infusion in Conscious Dogs. Circ Res. 1977;40:118-126.

31. Kubzansky LD, Kawachi I. Going to the heart of the matter. J Psychosom Res. 2000;48:323337.

32. Jorgensen RS, Kolodziej ME. Suppressed anger, evaluative threat, and cardiovascular reactivity: A tripartite profile approach. Int J Psychophysiol. 2007;66:102-108.

33. Jorgensen RS, Johnson BT, Kolodziej ME, Schreer GE. Elevated Blood Pressure and Personality: A Meta-Analytic Review. Psychol Bull. 1996;120:293-320.

34. Smith TW, Christensen AJ. Cardiovascular reactivity and interpersonal relations psychosomatic processes in social context. J Soc Clin Psychol. 1992;11:279-301.

35. Gerin W, Pickering TG, Glynn L, Christenfeld N, Schwartz A, Carroll D, Davidson K. An historical context for behavioral models of hypertension. J Psychosom Res. 2000;48:369-377. 
36. Matthews KA, Woodall KL, Allen MT. Cardiovascular Reactivity to Stress Predicts Future Blood-Pressure Status. Hypertension. 1993;22:479-485.

37. Chida Y, Steptoe A. Greater Cardiovascular Responses to Laboratory Mental Stress Are Associated With Poor Subsequent Cardiovascular Risk Status A Meta-Analysis of Prospective Evidence. Hypertension. 2010;55:1026-U368.

38. Virtanen R, Jula A, Salminen JK, VoipioPulkki L, Helenius H, Kuusela T, Airaksinen J. Anxiety and Hostility Are Associated With Reduced Baroreflex Sensitivity and Increased Beatto-Beat Blood Pressure Variability. Psychosom Med. 2003;65:751-756.

39. Hughes JW, Dennis MF, Beckham JC. Baroreceptor sensitivity at rest and during stress in women with posttraumatic stress disorder or major depressive disorder. J Trauma Stress. 2007;20:667-676.

40. Pilowsky PM, Goodchild AK. Baroreceptor reflex pathways and neurotransmitters: 10 years on. J Hypertens. 2002;20:1675-1688.

41. Ducher M, Fauvel JP, Cerutti C. Risk Profile in Hypertension Genesis: A Five-Year FollowUp Study. Am J Hypertens. 2006;19:775-780.

42. Dzielak DJ. The Immune System and Hypertension. Hypertension. 1992;19:36-44.

43. Khraibi AA. Association between Disturbances in the Immune-System and Hypertension. Am J Hypertens. 1991;4:635-641.

44. Bendich A, Belisle EH, Strausser HR. Immune system modulation and its effect on the blood pressure of the spontaneously hypertensive male and female rat. Biochem Biophys Res Commun. 1981;99:600-607.

45. Harrison DG, Vinh A, Lob H, Madhur MS. Role of the adaptive immune system in hypertension. Curr Opin Pharmacol. 2010;10:203-207.

46. Segerstrom SC, Miller GE. Psychological Stress and the Human Immune System: A MetaAnalytic Study of 30 Years of Inquiry. Psychol Bull. 2004;130:601-630. 
47. Jemmott JB, Locke SE. Psychosocial factors, immunologic mediation, and human susceptibility to infectious diseases: How much do we know? Psychol Bull. 1984;95:78-108.

48. Marsland AL, Sathanoori R, Muldoon MF, Manuck SB. Stimulated production of interleukin- 8 covaries with psychosocial risk factors for inflammatory disease among middleaged community volunteers. Brain Behav Immun. 2007;21:218-228.

49. Harrison DG, Guzik TJ, Lob HE, Madhur MS, Marvar PJ, Thabet SR, Vinh A, Weyand CM. Inflammation, immunity, and hypertension. Hypertension. 2011;57:132-140.

50. Dimsdale JE, Pierce C, Schoenfeld D, Brown A, Zusman R, Graham R. Suppressed anger and blood pressure: the effects of race, sex, social class, obesity, and age. Psychosom Med. 1986;48:430-436.

51. Cottington EM, Brock BM, House JS, Hawthorne VM. Psychosocial factors and blood pressure in the Michigan Statewide Blood Pressure Survey. Am J Epidemiol. 1985;121:515-529.

52. Gentry WD, Chesney AP, Gary HE, Hall RP, Harburg E. Habitual Anger-Coping Styles .1. Effect on Mean Blood-Pressure and Risk for Essential-Hypertension. Psychosom Med. 1982;44:195-202.

53. Harburg E, Erfurt J, Hauenste L, Chape C, Schull W, Schork M. Socio-Ecological Stress, Suppressed Hostility, Skin Color, and Black-White Male Blood-Pressure - Detroit. Psychosom Med. 1973;35:276-296.

54. Johnson EH. Cardiovascular reactivity, emotional factors, and home blood pressures in black males with and without a parental history of hypertension. Psychosom Med. 1989;51:390-403.

55. Johnson EH, Spielberger CD, Worden TJ, Jacobs GA. Emotional and Familial Determinants of Elevated Blood-Pressure in Black-And-White Adolescent Males. J Psychosom Res. 1987;31:287-300.

56. Johnson EH, Schork NJ, Speilberger CD. Emotional and Familial Determinants of Elevated Blood-Pressure in Black and White Adolescent Females. J Psychosom Res. 1987;31:731-741. 
57. Johnson EH. Anger and anxiety as determinants of elevated blood pressure in adolescents: The Tampa Study. [Ph.D.]. United States -- Florida: University of South Florida; 1984.

58. Schneider RH, Egan BM, Johnson EH, Drobny H, Julius S. Anger and anxiety in borderline hypertension. Psychosom Med. 1986;48:242-248.

59. Dembroski TM, Macdougall JM, Williams RB, Haney TL, Blumenthal JA. Components of Type-A, Hostility, and Anger-in - Relationship to Angiographic Findings. Psychosom Med. 1985;47:219-233.

60. Siegman AW, Dembroski TM, Ringel N. Components of Hostility and the Severity of Coronary-Artery Disease. Psychosom Med. 1987;49:127-135.

61. Julius M, Harburg E, Cottington EM, Johnson EH. Anger-Coping Types, Blood-Pressure, and All-Cause Mortality - a Follow-Up in Tecumseh, Michigan (1971-1983). Am J Epidemiol. 1986;124:220-233.

62. Everson S, Goldberg D, Kaplan G, Julkunen J, Salonen J. Anger expression and incident hypertension. Psychosom Med. 1998;60:730-735.

63. Everson SA, Kauhanen J, Kaplan GA, Goldberg DE, Julkunen J, Tuomilehto J, Salonen JT. Hostility and increased risk of mortality and acute myocardial infarction: the mediating role of behavioral risk factors. Am J Epidemiol. 1997;146:142-152.

64. Matthews KA, Glass DC, Rosenman RH, Bortner RW. Competitive Drive, Pattern-A, and Coronary Heart-Disease - further Analysis of some Data from Western Collaborative Group Study. J Chronic Dis. 1977;30:489-498.

65. Theorell T, Lind E, Floderus B. Relationship of Disturbing Life-Changes and Emotions to Early Development of Myocardial-Infarction and Other Serious Illnesses. Int J Epidemiol. 1975;4:281-293.

66. Crane RS. The role of anger, hostility and aggression in essential hypertension. [Ph.D.]. United States -- Florida: University of South Florida; 1981. 
67. Hosseini SH, Mokhberi V, Mohammadpour RA, Mehrabianfard M, Lashak NB. Anger expression and suppression among patients with essential hypertension. Int J Psychiatry Clin Pract. 2011;15:214-218.

68. Laude D, Girard A, Consoli S, Mounier-Vehier C, Elghozi JL. Anger Expression and Cardiovascular Reactivity to Mental Stress: A Spectral Analysis Approach. Clin Exp Hypertens. 1997;19:901-911.

69. Markovitz JH, Matthews KA, Wing RR, Kuller LH, Meilahn EN. Psychological, biological and health behavior predictors of blood pressure changes in middle-aged women. $J$ Hypertens. 1991;9:399-406.

70. Helmers KF, Baker B, O'Kelly B, Tobe SW. Anger expression, gender, and ambulatory blood pressure in mild, unmedicated adults with hypertension. Ann Behav Med. 2000;22:60-64.

71. Haynes S, Levine S, Scotch N, Feinleib M, Kannel W. Relationship of Psychosocial Factors to Coronary Heart-Disease in Framingham Study .1. Methods and Risk-Factors. Am J Epidemiol. 1978;107:362-383.

72. Lerman CE, Brody DS, Hui T, Lazaro C, Smith DG, Blum MJ. The white-coat hypertension response: prevalence and predictors. J Gen Intern Med. 1989;4:226-231.

73. Durel LA, Carver CS, Spitzer SB, Llabre MM. Associations of blood pressure with selfreport measures of anger and hostility among Black and White men and women. Health Psychol. 1989;8:557-575.

74. Player MS, King DE, Mainous AG, Geesey ME. Psychosocial factors and progression from prehypertension to hypertension or coronary heart disease. Ann Fam Med. 2007;5:403-411.

75. Rohrmann S, Bechtoldt MN, Hopp H, Hodapp V, Zapf D. Psychophysiological effects of emotional display rules and the moderating role of trait anger in a simulated call center. Anxiety Stress and Coping. 2011;24:421-438.

76. Hogan BE, Linden W. Curvilinear relationships of expressed anger and blood pressure in women but not in men: evidence from two samples. J Psychosom Res. 2005;59:97-102. 
77. Harburg E, Gleiberman L, Russell M, Cooper ML. Anger-coping styles and blood pressure in black and white males: Buffalo, New York. Psychosom Med. 1991;53:153-164.

78. Pollard TM, Pearce KL, Rousham EK, Schwartz JE. Do blood pressure and heart rate responses to perceived stress vary according to endogenous estrogen level in women?. Am J Phys Anthropol. 2007;132:151-157.

79. Kario K, James GD, Marion R, Ahmed M, Pickering TG. The influence of work- and homerelated stress on the levels and diurnal variation of ambulatory blood pressure and neurohumoral factors in employed women. Hypertens Res. 2002;25:499-506.

80. James GD, Bovbjerg DH. Age and perceived stress independently influence daily blood pressure levels and variation among women employed in wage jobs. Am J Human Biol. 2001;13:268-274.

81. Matthews KA, Owens JF, Allen MT, Stoney CM. Do cardiovascular responses to laboratory stress relate to ambulatory blood pressure levels? Yes, in some of the people, some of the time. Psychosom Med. 1992;54:686-697.

82. Langewitz W, Rüddel H, Von Eiff AW. Influence of perceived level of stress upon ambulatory blood-pressure, heart rate and respiratory frequency. J Clin Hypertens. 1987;3:743748.

83. Huang Y, Mai W, Hu Y, Wu Y, Song Y, Qiu R, Dong Y, Kuang J. Poor sleep quality, stress status, and sympathetic nervous system activation in nondipping hypertension. Blood Press Monit. 2011;16:117-123.

84. Wright BR, Barbosa-Leiker C, Hoekstra T. Law enforcement officer versus non-law enforcement officer status as a longitudinal predictor of traditional and emerging cardiovascular risk factors. J Occup Environ Med. 2011;53:730-734.

85. Rod NH, Gronbaek M, Schnohr P, Prescott E, Kristensen TS. Perceived stress as a risk factor for changes in health behaviour and cardiac risk profile: a longitudinal study. J Intern Med. 2009;266:467-475. 
86. Strickland OL, Giger JN, Nelson MA, Davis CM. The relationships among stress, coping, social support, and weight class in premenopausal African American women at risk for coronary heart disease. J Cardiovasc Nurs. 2007;22:272-278.

87. Strodl E, Kenardy J, Aroney C. Perceived stress as a predictor of the self-reported new diagnosis of symptomatic CHD in older women. Int J Behav Med. 2003;10:205-220.

88. Iso H, Date C, Yamamoto A, Toyoshima H, Tanabe N, Kikuchi S, Kondo T, Watanabe Y, Wada Y, Ishibashi T, Suzuki H, Koizumi A, Inaba Y, Tamakoshi A, Ohno Y, JACC Study Grp. Perceived mental stress and mortality from cardiovascular disease among Japanese men and women - The Japan Collaborative Cohort Study for Evaluation of Cancer Risk Sponsored by Monbusho (JACC Study). Circulation. 2002;106:1229-1236.

89. Din-Dzietham R, Nembhard WN, Collins R, Davis SK. Perceived stress following racebased discrimination at work is associated with hypertension in African-Americans. The metro Atlanta heart disease study, 1999-2001. Soc Sci Med. 2004;58:449-461.

90. Nielsen NR, Kristensen TS, Prescott E, Larsen KS, Schnohr P, Grønbœk M. Perceived Stress and Risk of Ischemic Heart Disease: Causation or Bias? Epidemiology. 2006;17:391-397.

91. Alexander F. Emotional Factors in Essential Hypertension. Psychosom Med. 1939;1:173179.

92. Funkenstein DH, King SH, Drolette M. The direction of anger during a laboratory stressinducing situation. Psychosom Med. 1954;16:404-413.

93. Spielberger CD, London P. Rage boomerangs: Lethal Type-A anger. American Health. 1982;1:52-56.

94. Harburg E, Blakelock EH,Jr, Roeper PR. Resentful and reflective coping with arbitrary authority and blood pressure: Detroit. Psychosom Med. 1979;41:189-202.

95. Lee-Feldstein A, Harburg E, Hauenstein L. Parity and blood pressure among four race-stress groups of females in Detroit. Am J Epidemiol. 1980;111:356-366. 
96. Harburg E, Schull WJ, Erfurt JC, Schork MA. A family set method for estimating heredity and stress-I. J Chronic Dis. 1970;23:69-81.

97. Schwartz GE, Weinberger DA, Singer JA. Cardiovascular differentiation of happiness, sadness, anger, and fear following imagery and exercise. Psychosom Med. 1981;43:343-364.

98. Diamond EL. The Role of Anger and Hostility in Essential-Hypertension and Coronary Heart-Disease. Psychol Bull. 1982;92:410-433.

99. Friedman R, Schwartz JE, Schnall PL, Landsbergis PA, Pieper C, Gerin W, Pickering TG. Psychological variables in hypertension: relationship to casual or ambulatory blood pressure in men. Psychosom Med. 2001;63:19-31.

100. Alexander F, French TM. Studies in Psychosomatic Medicine: An Approach to the Cause and Treament of Vegetative Disturbance. New York: Ronald Press Company; 1948.

101. Krantz DS, Manuck SB. Acute psychophysiologic reactivity and risk of cardiovascular disease: A review and methodologic critique. Psychol Bull. 1984;96:435-464.

102. Sullivan PA, Procci WR, Dequattro V, Schoentgen S, Levine D, Vandermeulen J, Bornheimer JF. Anger, Anxiety, Guilt and Increased Basal and Stress-Induced Neurogenic Tone - Causes Or Effects in Primary Hypertension. Clin Sci. 1981;61:S389-S392.

103. Spielberger CD, Jacobs GA, Russell SF, Crane RS. Assessment of anger: The state-trait anger scale. In: Butcher JN, Spielberger CD, eds. Advances in Personality Assessment. Hillsdale, N.J.: Lawrence Erlbaum Associates; 1983:161-190.

104. Spielberger CD, Johnson EH, Russell SF, Crane RH, Worden TJ. The experience and expression of anger: Construction and validation of an anger expression scale. In: Chesney MA, Rosenman RH, eds. Anger and Hostility in Cardiovascular and Behavioral Disorders. New York: Hemisphere-McGraw Hill; 1985:5-30.

105. Averill JR. Studies on anger and aggression: Implications for theories of emotion. Am Psychol. 1983;38:1145-1160. 
106. Lopez FG, Thurman CW. A Cognitive-Behavioral Investigation of Anger among CollegeStudents. Cognit Ther Res. 1986;10:245-256.

107. Eckhardt CI, Kassinove H, Tsytsarev SV, Sukhodolsky DG. A Russian Version of the State-Trait Anger Expression Inventory: Preliminary Data. J Pers Assess. 1995;64:440.

108. Moscoso M, Spielberger C. Measuring the experience, expression and control of anger in Latin America: The Spanish Multi-Cultural State-Trait Anger Expression Inventory. Revista Interamericana De Psicologia. 1999;33:29-48.

109. Carels RA, Sherwood A, Blumenthal JA. Psychosocial influences on blood pressure during daily life. Int J Psychophysiol. 1998;28:117-129.

110. Webb MS, Beckstead JW. Stress-related influences on blood pressure in African American women. Res Nurs Health. 2002;25:383-393.

111. Kotchen JM, McKean HE, Kotchen TA. Blood pressure trends with aging. Hypertension. 1982;4:128-134.

112. Kearney PM, Whelton M, Reynolds K, Whelton PK, He J. Worldwide prevalence of hypertension: a systematic review. J Hypertens. 2004;22:11-19.

113. Himmelmann A, Svensson A, Hansson L. Influence of sex on blood pressure and left ventricular mass in adolescents: the Hypertension in Pregnancy Offspring Study. J Hum Hypertens. 1994;8:485-490.

114. Schenck-Gustafsson K. Risk factors for cardiovascular disease in women: assessment and management. Eur Heart J. 1996;17:2-8.

115. Ohira T, Tanigawa $\mathrm{T}$, Iso $\mathrm{H}$, Sankai $\mathrm{T}$, Imano $\mathrm{H}$, Shimamoto $\mathrm{T}$. Impact of anger expression on blood pressure levels in white-color workers with low-coping behavior. Environ Health Prev Med. 2000;5:37-42.

116. Douglas J, Bakris G, Epstein M, Ferdinand K, Ferrario C, Flack J, Jamerson K, Jones W, Haywood J, Maxey R, Ofili E, Sanders E, Schiffrin E, Sica D, Sowers J, Vidt D, Hypertension African Amer Working. Management of high blood pressure in African Americans - Consensus 
statement of the hypertension in African Americans Working Group of the International Society on Hypertension in Blacks. Arch Intern Med. 2003;163:525-541.

117. Forgays D, Spielberger C, Ottaway S, Forgays D. Factor Structure of the State-Trait Anger Expression Inventory for Middle-Aged Men and Women. Assessment. 1998;5:141-155.

118. Porter LS, Stone AA, Schwartz JE. Anger Expression and Ambulatory Blood Pressure: A Comparison of State and Trait Measures. Psychosom Med. 1999;61:454-463.

119. Shapiro D, Jamner LD, Goldstein IB. Ambulatory stress psychophysiology: the study of "compensatory and defensive counterforces" and conflict in a natural setting. Psychosom Med. 1993;55:309-323.

120. Bishop GD, Pek J, Ngau F. Ethnicity, sex, trait anger, and nocturnal blood pressure decline. Psychophysiology. 2005;42:290-297.

121. Jamner LD, Shapiro D, Hui KK, Oakley ME, Lovett M. Hostility and differences between clinic, self-determined, and ambulatory blood pressure. Psychosom Med. 1993;55:203-211.

122. Jamner LD, Shapiro D, Goldstein IB, Hug R. Ambulatory blood pressure and heart rate in paramedics: effects of cynical hostility and defensiveness. Psychosom Med. 1991;53:393-406.

123. Steele MS, Mcgarvey ST, Mph. Anger Expression, Age, and Blood Pressure in Modernizing Samoan Adults. Psychosom Med. 1997;59:632-637.

124. Campbell NR, Hemmelgarn BR. New recommendations for the use of ambulatory blood pressure monitoring in the diagnosis of hypertension. CMAJ. 2012;184:663-664.

125. Campbell NR, Myers MG, McKay DW. Is usual measurement of blood pressure meaningful? Blood Press Monit. 1999;4:71-76.

126. Lazarus RS. Psychological Stress and the Coping Process. New York: McGraw-Hill; 1966.

127. Lazarus RS. Psychological stress and coping in adaptation and illness. In: Lipowski ZJ, Lipsi DR, Whybrow PC, eds. Psychosomatic Medicine: Current Trends and Clinical Applications. New York: Oxford University Press; 1977:14-26. 
128. Lazarus RS. Stress, Appraisal, and Coping. New York: Springer Pub. Co.; 1984.

129. Cohen S, Kamarck T, Mermelstein R. A global measure of perceived stress. J Health Soc Behav. 1983;24:385-396.

130. Kobasa SC. Stressful Life Events, Personality, and Health - Inquiry into Hardiness. J Pers Soc Psychol. 1979;37:1-11.

131. Cohen S. Contrasting the Hassles Scale and the Perceived Stress Scale - Whos really Measuring Appraised Stress. Am Psychol. 1986;41:716-718.

132. Folkman S, Lazarus RS. An Analysis of Coping in a Middle-Aged Community Sample. $J$ Health Soc Behav. 1980;21:219-239.

133. Kanner AD, Coyne JC, Schaefer C, Lazarus RS. Comparison of 2 Modes of Stress Measurement Daily Hassles and Uplifts Vs. Major Life Events. J Behav Med. 1981;4:1-40.

134. Logan JG, Barksdale DJ, Carlson J, Carlson BW, Rowsey PJ. Psychological stress and arterial stiffness in Korean Americans. J Psychosom Res. 2012;73:53-58.

135. Ginty AT, Conklin SM. High perceived stress in relation to life events is associated with blunted cardiac reactivity. Biol Psychol. 2011;86:383-385.

136. Kario K, Matsuo T, Kobayashi H, Imiya M, Matsuo M, Shimada K. Nocturnal fall of blood pressure and silent cerebrovascular damage in elderly hypertensive patients: Advanced silent cerebrovascular damage in extreme dippers. Hypertension. 1996;27:130-135.

137. Shimada K, Kawamoto A, Matsubayashi K, Nishinaga M, Kimura S, Ozawa T. Diurnal blood pressure variations and silent cerebrovascular damage in elderly patients with hypertension. J Hypertens. 1992;10:875-878.

138. Verdecchia P, Porcellati C, Schillaci G, Borgioni C, Ciucci A, Battistelli M, Guerrieri M, Gatteschi C, Zampi I, Santucci A, Santucci C, Reboldi G. Ambulatory blood pressure: An independent predictor of prognosis in essential hypertension. Hypertension. 1994;24:793-801. 
139. Hoshide S, Kario K, Hoshide Y, Umeda Y, Hashimoto T, Kunii O, Ojima T, Shimada K. Associations between nondipping of nocturnal blood pressure decrease and cardiovascular target organ damage in strictly selected community-dwelling normotensives. Am J Hypertens. 2003;16:434-438.

140. Moya-Albiol L, Serrano MÁ, Salvador A. Burnout as an important factor in the psychophysiological responses to a work day in Teachers. Stress Health. 2010;26:382-393.

141. Koizumi M, Kaneko Y, Tosa S, Watanabe H, Kosaka T, Hasegawa H, Motohashi Y, Ito H. Association between perceived stress and plasma B-type natriuretic peptide concentrations. Circ J. 2009;73:1055-1061.

142. Suter PM, Maire R, Holtz D, Vetter W. Relationship between self-perceived stress and blood pressure. J Hum Hypertens. 1997;11:171-176.

143. Strayhorn G. Social supports, perceived stress, and health: the black experience in medical school--a preliminary study. J Natl Med Assoc. 1980;72:869-881.

144. Davidson K, MacGregor MW, Stuhr J, Gidron Y. Increasing constructive anger verbal behavior decreases resting blood pressure: A secondary analysis of a randomized controlled hostility intervention. Int J Behav Med. 1999;6:268-278.

145. Davison GC, Williams ME, Nezami E, Bice TL, DeQuattro VL. Relaxation, reduction in angry articulated thoughts, and improvements in borderline hypertension and heart rate. J Behav Med. 1991;14:453-468.

146. Linden W, Lenz JW, Con AH. Individualized stress management for primary hypertension: a randomized trial. Arch Intern Med. 2001;161:1071-1080.

147. Larkin KT, Zayfert C. Anger management training with mild essential hypertensive patients. J Behav Med. 1996;19:415-433.

148. Bennett P, Wallace L, Carroll D, Smith N. Treating Type A behaviours and mild hypertension in middle-aged men. J Psychosom Res. 1991;35:209-223. 
149. McCraty R, Atkinson M, Tomasino D. Impact of a Workplace Stress Reduction Program on blood pressure and emotional health in hypertensive employees. J Altern Complement Med. 2003;9:355-369.

150. Kabat-Zinn J, Lipworth L, Burney R. The clinical use of mindfulness meditation for the self-regulation of chronic pain. J Behav Med. 1985;8:163-190.

151. Shapiro SL, Schwartz GE, Bonner G. Effects of mindfulness-based stress reduction on medical and premedical students. J Behav Med. 1998;21:581-599.

152. Astin JA. Stress reduction through mindfulness meditation. Effects on psychological symptomatology, sense of control, and spiritual experiences. Psychother Psychosom. 1997;66:97-106.

153. Kabat-Zinn J, Massion AO, Kristeller J, Peterson LG, Fletcher KE, Pbert L, Lenderking WR, Santorelli SF. Effectiveness of a meditation-based stress reduction program in the treatment of anxiety disorders. Am J Psychiatry. 1992;149:936-943.

154. Vieten C, Astin J. Effects of a mindfulness-based intervention during pregnancy on prenatal stress and mood: results of a pilot study. Arch Womens Ment Health. 2008;11:67-74.

155. Vollestad J, Sivertsen B, Nielsen GH. Mindfulness-based stress reduction for patients with anxiety disorders: evaluation in a randomized controlled trial. Behav Res Ther. 2011;49:281-288.

156. Barnhofer T, Duggan D, Crane C, Hepburn S, Fennell MJ, Williams JM. Effects of meditation on frontal alpha-asymmetry in previously suicidal individuals. Neuroreport. 2007;18:709-712.

157. Kenny MA, Williams JM. Treatment-resistant depressed patients show a good response to Mindfulness-based Cognitive Therapy. Behav Res Ther. 2007;45:617-625.

158. Kingston J, Chadwick P, Meron D, Skinner TC. A pilot randomized control trial investigating the effect of mindfulness practice on pain tolerance, psychological well-being, and physiological activity. J Psychosom Res. 2007;62:297-300. 
159. Sephton SE, Salmon P, Weissbecker I, Ulmer C, Floyd A, Hoover K, Studts JL. Mindfulness meditation alleviates depressive symptoms in women with fibromyalgia: results of a randomized clinical trial. Arthritis Rheum. 2007;57:77-85.

160. Grossman P, Tiefenthaler-Gilmer U, Raysz A, Kesper U. Mindfulness training as an intervention for fibromyalgia: evidence of postintervention and 3-year follow-up benefits in well-being. Psychother Psychosom. 2007;76:226-233.

161. Schmidt S, Grossman P, Schwarzer B, Jena S, Naumann J, Walach H. Treating fibromyalgia with mindfulness-based stress reduction: results from a 3-armed randomized controlled trial. Pain. 2011;152:361-369.

162. Kristeller JL, Hallett CB. An Exploratory Study of a Meditation-based Intervention for Binge Eating Disorder. J Health Psychol. 1999;4:357-363.

163. Kabat-Zinn J, Wheeler E, Light T, Skillings A, Scharf MJ, Cropley TG, Hosmer D, Bernhard JD. Influence of a mindfulness meditation-based stress reduction intervention on rates of skin clearing in patients with moderate to severe psoriasis undergoing phototherapy (UVB) and photochemotherapy (PUVA). Psychosom Med. 1998;60:625-632.

164. Diebold JC. Mindfulness in the machine: A mindfulness-based cognitive therapy for the reduction of driving anger. [Ph.D.]. United States -- New York: Hofstra University; 2003.

165. Silva JM. Mindfulness based cognitive therapy for the reduction of anger in married men. [Ph.D.]. United States -- New York: Hofstra University; 2006.

166. Wright S, Day A, Howells K. Mindfulness and the treatment of anger problems. Aggression and Violent Behavior. 2009;14:396-401.

167. Lane JD, Seskevich JE, Pieper CF. Brief meditation training can improve perceived stress and negative mood. Altern Ther Health Med. 2007;13:38-44.

168. Chang VY, Palesh O, Caldwell R, Glasgow N, Abramson M, Luskin F, Gill M, Burke A, Koopman C. The effects of a mindfulness-based stress reduction program on stress, mindfulness self-efficacy, and positive states of mind. Stress Health. 2004;20:141-147. 
169. Oman D, Shapiro SL, Thoresen CE, Plante TG, Flinders T. Meditation Lowers Stress and Supports Forgiveness Among College Students: A Randomized Controlled Trial. J Am Coll Health. 2008;56:569-578.

170. Branstrom R, Kvillemo P, Brandberg Y, Moskowitz JT. Self-report Mindfulness as a Mediator of Psychological Well-being in a Stress Reduction Intervention for Cancer Patients-A Randomized Study. Ann Behav Med. 2010;39:151-161.

171. Klatt M, Buckworth J, Malarkey W. Effects of Low-Dose Mindfulness-Based Stress Reduction (MBSR-ld) on Working Adults. Health Educ Behav. 2009;36:601-614.

172. Nyklicek I, Kuijpers KF. Effects of mindfulness-based stress reduction intervention on psychological well-being and quality of life: Is increased mindfulness indeed the mechanism? Ann Behav Med. 2008;35:331-340.

173. Borders A, Liang CTH. Rumination Partially Mediates the Associations Between Perceived Ethnic Discrimination, Emotional Distress, and Aggression. Cultur Divers Ethnic Minor Psychol. 2011;17:125-133.

174. Borders A, Earleywine M, Jajodia A. Could mindfulness decrease anger, hostility, and aggression by decreasing rumination? Aggr Behav. 2010;36:28-44.

175. Feltman R, Robinson MD, Ode S. Mindfulness as a moderator of neuroticism-outcome relations: A self-regulation perspective. J Res Pers. 2009;43:953-961.

176. Kabat-Zinn J. Full Catastrophe Living: Using the Wisdom of Your Body and Mind to Face Stress, Pain and Illness. New York: Delta; 1990.

177. Bishop SR. What do we really know about mindfulness-based stress reduction? Psychosom Med. 2002;64:71-83.

178. Kabat-Zinn J. An outpatient program in behavioral medicine for chronic pain patients based on the practice of mindfulness meditation: theoretical considerations and preliminary results. Gen Hosp Psychiatry. 1982;4:33-47. 
179. Bishop G, Quah S. Reliability and validity of measures of anger/hostility in Singapore: Cook \& Medley Ho Scale, STAXI and Buss-Durkee hostility inventory. Pers Individ Dif. 1998;24:867-878.

180. Jacobs GA, Latham LE, Brown MS. Test-retest reliability of the state-trait personality inventory and the anger expression scale. Anxiety Res. 1988;1:263-265.

181. Buss AH, Durkee A. An Inventory for Assessing Different Kinds of Hostility. J Consult Psychol. 1957;21:343-349.

182. Walter W. Cook, Donald M. Medley. Proposed hostility and Pharisaic-virtue scales for the MMPI. J Appl Psychol. 1954;38:414-418.

183. Schultz SD. A differentiation of several forms of hostility by scales empirically constructed from significant items on the Minnesota Multiphasic Personality Inventory. [Ph.D.]. United States -- Pennsylvania: The Pennsylvania State University; 1954.

184. Hathaway SR. Minnesota Multiphasic Personality Inventory;: Manual [by] S.R. Hathaway and J.C. McKinley. Rev. ed. New York: The Psychological Corporation; 1967.

185. Eysenck HJ. Manual of the Eysenck Personality Inventory. London: University of London Press; 1964.

186. Spielberger CD, Barker LR, Russell SF, Crane RS, Westberry LG, Knight G, Marks E. The preliminary manual for the State Trait Personality Inventory (STPI). Unpublished Manual, University of South Florida, Tampa. 1979.

187. Cohen S, Williamson GM. Perceived stress in a probability sample of the united-states. In: Spacapan S, Oskamp S, eds. The Social Psychology of Health. Newbury Park, CA: Sage; 1988:67.

188. Machulda MM, Bergquist TF, Ito V, Chew S. Relationship Between Stress, Coping, and Postconcussion Symptoms in a Healthy Adult Population. Arch Clin Neuropsychol. 1998;13:415-424. 
189. Hewitt P, Flett G, Mosher S. The Perceived Stress Scale - Factor Structure and Relation to Depression Symptoms in a Psychiatric Sample. J Psychopathol Behav Assess. 1992;14:247-257.

190. Pruessner J, Hellhammer D, Kirschbaum C. Burnout, perceived stress, and cortisol responses to awakening. Psychosom Med. 1999;61:197-204.

191. Ebrecht M, Hextall J, Kirtley L, Taylor A, Dyson M, Weinman J. Perceived stress and cortisol levels predict speed of wound heating in healthy male adults. Psychoneuroendocrinology. 2004;29:798-809.

192. Chang E. Does dispositional optimism moderate the relation between perceived stress and psychological well-being?: a preliminary investigation. Pers Individ Dif. 1998;25:233-240.

193. Dyck D, Short R, Vitaliano P. Predictors of burden and infectious illness in schizophrenia caregivers. Psychosom Med. 1999;61:411-419.

194. Hamarat E, Thompson D, Zabrucky K, Steele D, Matheny K, Aysan F. Perceived stress and coping resource availability as predictors of life satisfaction in young, middle-aged, and older adults. Exp Aging Res. 2001;27:181-196.

195. Pickering T, Shimbo D, Haas D. Current concepts: Ambulatory blood-pressure monitoring. N Engl J Med. 2006;354:2368-2374.

196. Kamarck T, Shiffman S, Smithline L, Goodie J, Paty J, Gnys M, Jong J. Effects of task strain, social conflict, and emotional activation on ambulatory cardiovascular activity: Daily life consequences of recurring stress in a multiethnic adult sample. Health Psychol. 1998;17:17-29.

197. Spielberger CD, Krasner SS, Solomon EP. The experience, expression and control of anger. In: Janisse MP, ed. Health Psychology: Individual Differences and Stress. New York: Springer/Verlag; 1988:89-108.

198. Vogele C, Jarvis A, Cheeseman K. Anger suppression, reactivity, and hypertension risk: Gender makes a difference. Ann Behav Med. 1997;19:61-69.

199. Cronbach LJ. Coefficient Alpha and the Internal Structure of Tests. Psychometrika. 1951;16:297-334. 
200. Tobe S, Kiss A, Szalai J, Perkins N, Tsigoulis M, Baker B. Impact of job and marital strain on ambulatory blood pressure. Am J Hypertens. 2005;18:1046-1051.

201. Baker B, Paquette M, Szalai JP, Driver H, Perger T, Helmers K, O'Kelly B, Tobe S. The influence of marital adjustment on 3-year left ventricular mass and ambulatory blood pressure in mild hypertension. Arch Intern Med. 2000;160:3453-3458.

202. Tobe SW, Kiss A, Sainsbury S, Jesin M, Geerts R, Baker B. The impact of job strain and marital cohesion on ambulatory blood pressure during 1 year: the double exposure study. Am $J$ Hypertens. 2007;20:148-153.

203. Wilder J. Basimetric Approach (Law of Initial Value) to Biological Rhythms. Ann N Y Acad Sci. 1962;98:1211-\&.

204. Nunnally JC, Bernstein IH. Psychometric Theory. 3rd ed. New York: McGraw-Hill; 1994. 205. Cohen J. Statistical Power Analysis for the Behavioral Sciences. 2nd ed. ed. Hillsdale, NJ: L. Erlbaum Associates; 1988.

206. Haynes RB, Taylor DW, Sackett DL, Gibson ES, Bernholz CD, Mukherjee J. Can Simple Clinical Measurements Detect Patient Non-Compliance. Hypertension. 1980;2:757-764.

207. Logan A, Achber C, Milne B, Campbell W, Haynes RB. Work-site treatment of hypertension by specially trained nurses. A controlled trial. Lancet. 1979;314:1175-1178.

208. Feldman R, Bacher M, Campbell N, Drover A, Chockalingam A. Adherence to pharmacologic management of hypertension. CJPH. 1998;89:I16-8.

209. Lindquist TL, Beilin LJ, Knuiman MW. Influence of lifestyle, coping, and job stress on blood pressure in men and women. Hypertension. 1997;29:1-7.

210. Daubenmier JJ, Weidner G, Sumner MD, Mendell N, Merritt-Worden T, Studley J, Ornish D. The Contribution of Changes in Diet, Exercise, and Stress Management to Changes in Coronary Risk in Women and Men in the Multisite Cardiac Lifestyle Intervention Program. Ann Behav Med. 2007;33:57-68. 
211. Guagnano MT, Ballone E, Colagrande V, Della Vecchia R, Manigrasso MR, Merlitti D, Riccioni G, Sensi S. Large waist circumference and risk of hypertension. Int J Obes. 2001;25:1360-1364.

212. Sesso HD, Cook NR, Buring JE, Manson JE, Gaziano JM. Alcohol consumption and the risk of hypertension in women and men. Hypertension. 2008;51:1080-1087.

213. Stranges S, Wu T, Dorn J, Freudenheim J, Muti P, Farinaro E, Russell M, Nochajski T, Trevisan M. Relationship of alcohol drinking pattern to risk of hypertension - A populationbased study. Hypertension. 2004;44:813-819.

214. Fuchs SC, Ferreira-Da-Silva AL, Moreira LB, Neyeloff JL, Fuchs FC, Gus M, Wiehe M, Fuchs FD. Efficacy of isolated home blood pressure monitoring for blood pressure control: Randomized controlled trial with ambulatory blood pressure monitoring-MONITOR study. $J$ Hypertens. 2012;30:75-80.

215. Harmon RL, Myers MA. Prayer and meditation as medical therapies. Phys Med Rehabil Clin N Am. 1999;10:651-662.

216. Rausch SM, Auerbach SM, Gramling SE. Gender and Ethnic Differences in Stress Reduction, Reactivity, and Recovery. Sex Roles. 2008;59:726-737.

217. Bowers R, Darner RM, Goldner CL, Sohnle S. Gender differences for recalled relaxation states, dispositions, beliefs and benefits. In: Smith JC, ed. Advances in ABC Relaxation Research : Applications and Inventories. New York: Springer Pub.; 2001:111-114.

218. Smith JC, McDuffie SR, Ritchie T, Holmes RH. Ethnic and racial differences in relaxation states for recalled relaxation activites. In: Smith JC, ed. Advances in ABC Relaxation Research : Applications and Inventories. New York: Springer Pub.; 2001:115-116.

219. Samuelson M, Carmody J, Kabat-Zinn J, Bratt M. Mindfulness-Based Stress Reduction in Massachusetts Correctional Facilities. The Prison Journal. 2007;87:254-268.

220. Denton M, Prus S, Walters V. Gender differences in health: a Canadian study of the psychosocial, structural and behavioural determinants of health. Soc Sci Med. 2004;58:25852600. 
221. Taylor SE, Klein LC, Lewis BP, Gruenewald TL, Gurung RAR, Updegraff JA. Biobehavioral Responses to Stress in Females: Tend-and-Befriend, Not Fight-or-Flight. Psychol Rev. 2000;107:411-429.

222. Barnett AG, van der Pols JC, Dobson AJ. Regression to the mean: what it is and how to deal with it. Int J Epidemiol. 2005;34:215-220.

223. Sterne J, White IR, Carlin JB, Spratt M, Royston P, Kenward MG, Wood AM, Carpenter JR. Multiple imputation for missing data in epidemiological and clinical research; potential and pitfalls. BMJ. 2009;339:157.

224. Gulliksson M, Burell G, Vessby B, Lundin L, Toss H, Svardsudd K. Randomized Controlled Trial of Cognitive Behavioral Therapy vs Standard Treatment to Prevent Recurrent Cardiovascular Events in Patients With Coronary Heart Disease Secondary Prevention in Uppsala Primary Health Care Project (SUPRIM). Arch Intern Med. 2011;171:134-140.

225. Suls J, Wan CK, Costa PT,Jr. Relationship of trait anger to resting blood pressure: a metaanalysis. Health Psychol. 1995;14:444-456. 